\title{
Aktualisierte Leitlinie zur Diagnostik und Therapie der Colitis ulcerosa 2011 - Ergebnisse einer Evidenzbasierten Konsensuskonferenz AWMF-Registriernummer: 021/009
}

Updated German Guideline on Diagnosis and Treatment of Ulcerative Colitis, 2011

Authors

Affiliations
A. Dignass ${ }^{1}$, J. C. Preiß ${ }^{2}$, D. E. Aust ${ }^{3}$, F. Autschbach ${ }^{4}$, A. Ballauff ${ }^{5}$, G. Barretton ${ }^{3}$, B. Bokemeyer ${ }^{6}$, S. Fichtner-Feigl ${ }^{7}$, S. Hage ${ }^{8}$, K. R. Herrlinger ${ }^{9}$, G. Jantschek ${ }^{10}$, A. Kroesen ${ }^{11}$, W. Kruis ${ }^{12}$, T. Kucharzik ${ }^{13}$, J. Langhorst ${ }^{14}$, M. Reinshagen ${ }^{15}$, G. Rogler ${ }^{16}$, D. Schleiermacher ${ }^{17}$, C. Schmidt ${ }^{8}$, S. Schreiber ${ }^{18}$, H. Schulze ${ }^{1}$, E. Stange ${ }^{9}$, M. Zeitz ${ }^{2}$, J. C. Hoffmann ${ }^{17}$, A. Stallmach ${ }^{8}$

Die Institutsangaben sind am Ende des Beitrags gelistet.
Bibliography

DOI http://dx.doi.org/10.1055/

s-0031-1281666

Published online 24.8.2011

Z Gastroenterol 2011; 49:

1276-1341 @ Georg Thieme

Verlag KG Stuttgart · New York ·

ISSN 0044-2771

\section{Correspondence}

Prof. Dr. Axel Dignass

FEBG, AGAF, Medizinische

Klinik I, Agaplesion Markus

Krankenhaus

Wilhelm-Epstein-Str. 4

60431 Frankfurt/Main

Tel.: ++49/69/95332201

Fax: ++49/69/95332291

axel.dignass@fdk.info

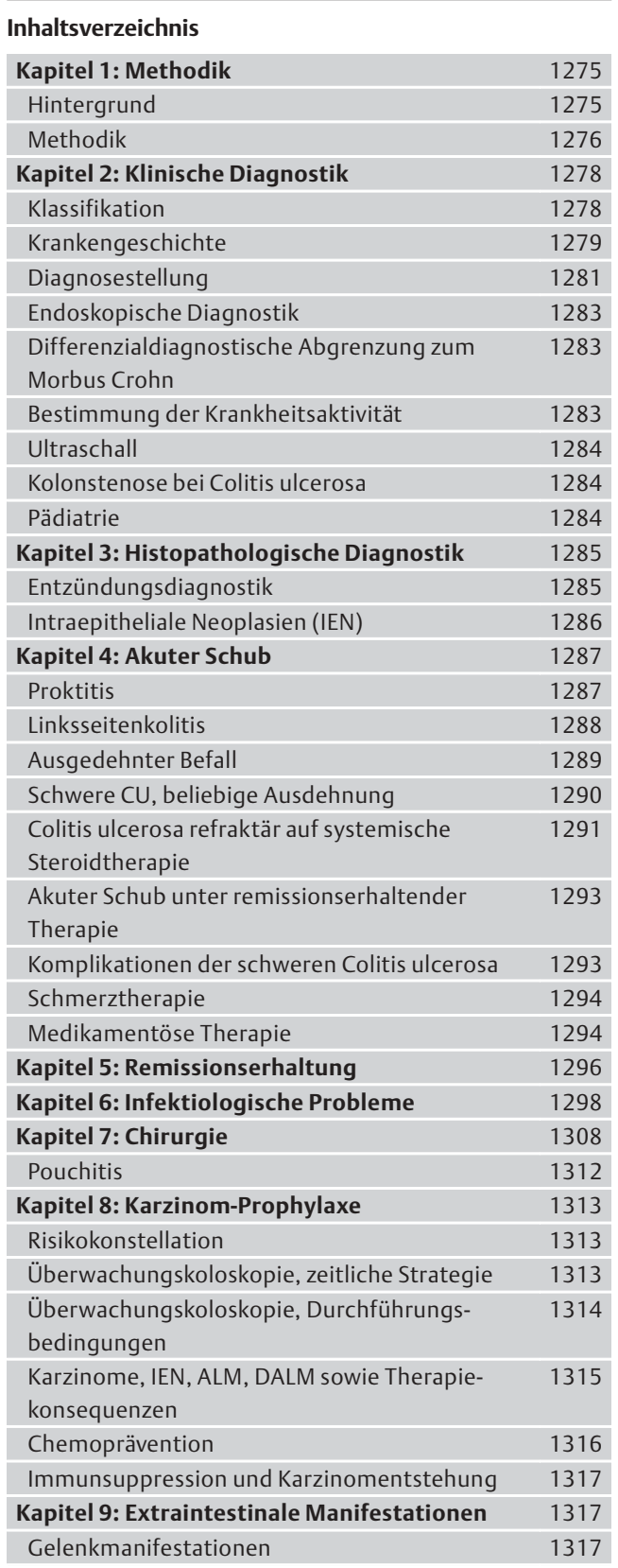

\begin{tabular}{lr}
\hline $\begin{array}{l}\text { Inhaltsverzeichnis (Fortsetzung) } \\
\text { Manifestation an der Leber und an den Gallenwe- }\end{array}$ & 1318 \\
gen & 1319 \\
\hline $\begin{array}{l}\text { Hautmanifestationen bei Colitis ulcerosa } \\
\text { Augenmanifestationen bei Colitis ulcerosa }\end{array}$ & 1319 \\
\hline $\begin{array}{l}\text { Osteoporose } \\
\text { Seltene extraintestinale Manifestationen und } \\
\text { extraintestinale Begleiterkrankungen }\end{array}$ & 1319 \\
\hline $\begin{array}{l}\text { Kapitel 10: Psychosomatik, Komplementär- } \\
\text { und Alternativmedizin }\end{array}$ & 1320 \\
\hline $\begin{array}{l}\text { Psychosomatik } \\
\text { Komplementär- und Alternativmedizin }\end{array}$ & 1320 \\
\hline
\end{tabular}

\section{Kapitel 1: Methodik}

\section{Hintergrund}

Die Colitis ulcerosa (CU) ist neben dem Morbus Crohn die wichtigste chronisch-entzündliche Darmerkrankung (CED). Die Inzidenz für die Colitis ulcerosa liegt in Deutschland bei bei 3,0-3,9 pro 100000 Einwohner [1, 2]. Die Prävalenz dürfte in der westlichen Welt derzeit bei 160 250 pro 100000 Einwohner liegen [3, 4]. Der höchste Gipfel der alterspezifischen Inzidenz liegt bei den 16- bis 25-Jährigen, wenn auch die Verteilung über die Altersdekaden weit gleichmäßiger ist als früher beschrieben wurde [2]. Somit beginnt für die meisten Patienten ihre Erkrankung während der Schulzeit oder der Berufausbildung und dauert während ihres gesamten beruflichen Lebens an. Daraus folgt, dass durch die Erkrankung nicht nur direkte Kosten (Medikamente, Arztbesuche, Operationen, Krankenhausaufenthalte etc.), sondern auch umfangreiche indirekte Kosten (Rente, Arbeitsausfälle etc.) entstehen. Es ist davon auszugehen, dass bei Patienten mit CU ca. die Hälfte der Gesamtkosten den indirekten Kosten zuzuordnen sind [5]. An direkten medizinischen Kosten wurden dabei zuletzt zwischen 2500 und $5000 €$ pro 
Jahr für einen Patienten mit Colitis ulcerosa errechnet. Insgesamt entfielen 75\% der direkten Kosten allein auf Arzneimittelkosten [5, 6]. Hochgerechnet werden allein in Deutschland ca. 1-2 Milliarden $€$ pro Jahr für die Versorgung von Patienten mit Colitis ulcerosa aufgewandt.

Eine besondere Untergruppe von Patienten mit Colitis ulcerosa sind Kinder und Jugendliche. Bei 15-25\% der Patienten treten die ersten Symptome vor dem 20. Lebensjahr auf, vereinzelt beginnt die Erkrankung im Säuglingsalter. Andere Differenzialdiagnosen, v.a. angeborene Immundefekterkrankungen und eine allergische Kolitis, sind auszuschließen. Die Behandlung von Kindern und Jugendlichen vor und während der Pubertät bis zum Abschluss des Wachstums sollte durch den in der Gastroenterologie spezialisierten Facharzt für Kinder- und Jugendmedizin erfolgen.

Der klinische Verlauf und die Ausdehnung der Erkrankung sowie das Ausmaß an extraintestinalen Manifestationen sind sehr variabel. Daraus ergibt sich die Notwendigkeit einer komplexen Diagnostik und einer abgestuften Therapie in der klinischen Praxis. Die jetzt überarbeitete Leitlinie basiert wesentlich auf der Leitlinie der Deutschen Gesellschaft für Verdauungs- und Stoffwechselkrankheiten (DGVS) aus dem Jahr 2004 sowie der Leitlinie der European Crohn's and Colitis Organization (ECCO) von 2008 [7-10]. Die überarbeitete Leitlinie wird in 10 Abschnitten dargestellt, wobei im ersten Kapitel die Ziele und methodischen Grundlagen der Leitlinie dargestellt werden, während in den nachfolgenden neun Kapiten die evidenzbasierten Empfehlungen in unterschiedlichen diagnostischen und klinischen Szenarien dargestellt werden.

\section{Ziele der Leitlinie und Gültigkeitsdauer}

Ziel dieser Leitlinie soll eine praxisorientierte Zusammenfassung und Bewertung des gegenwärtigen Wissensstands zur Diagnostik und Therapie der Colitis ulcerosa bei pädiatrischen Patienten und Erwachsenen sein. Sie soll einen Handlungskorridor für die häufigsten Entscheidungen liefern. Sie soll zudem der evidenzbasierten Fort- und Weiterbildung dienen und somit eine Verbesserung der medizinischen Versorgung von Patienten mit Colitis ulcerosa in der ambulanten und stationären Versorgung erreichen.

Die Leitlinie richtet sich an alle an der Diagnostik und Therapie beteiligten Berufsgruppen (Allgemeinmediziner, Internisten, Kinder- und Jugendmediziner, Chirurgen, Pathologen) sowie Betroffene und Leistungserbringer (Krankenkassen, Rentenversicherungsträger).

Die Struktur der Leitlinie orientiert sich am Deutschen Instrument zur methodischen Leitlinien-Bewertung (DELBI) und entspricht den Anforderungen der AWMF an eine S3-Leitlinie [11]. Details zur Methodik sind in einem separaten Methodenreport dargestellt.

Die Gültigkeit dieser überarbeiteten Leitlinie wird auf 5 Jahre geschätzt, sodass die Revision für 2016 geplant ist.

\section{Methodik}

\section{Organisatorischer Ablauf des Konsensusprozesses}

Basierend auf dem Teilnehmerkreis der früheren Konsensuskonferenzen erstellte ein Koordinationskomitee (A. Dignass, A. Stallmach und J.C. Hoffmann) in Abstimmung mit einer Patientenvertreterin (B. Kaltz) eine Teilnehmerliste für insgesamt 10 Arbeitsgruppen ( Tab.1). Dabei erfolgte die Auswahl unter der Berücksichtigung folgender Kriterien:
1. Fachliche Expertise

2. Für Ärzte: Schlüsseldisziplin (Innere Medizin, Gastroenterologie, Epidemiologie, Ernährungsmedizin, pädiatrische Gastroenterologie, Pathologie, Komplementäre Medizin, Psychosomatik, Radiologie, Viszeralchirurgie)

3. Für Ärzte: Versorgungsstufe (Niedergelassene, Krankenhaus der Grund-, Schwerpunkt- und Maximalversorgung)

4. Teilnehmerart: Ärzte, Patienten

Jede der neben DGVS beteiligten Fachgesellschaften benannte ebenfalls Vertreter. Alle Mitglieder der Leitliniengruppe gaben Informationen zu potenziellen Interessenkonflikten ab. Diese wurden von den Koordinatoren gesichtet, dabei ergab sich kein Hinweis auf Interessenkonflikte, die zu einer unzulässigen Beeinflussung der Leitlinienerstellung hätten führen können. Der zeitliche Ablauf des Konsensusprozesses ist in Tab.2 dargestellt. Das methodische Vorgehen wurde am 19.4.2009

\section{Tab. 1 Mitglieder der Leitliniengruppe.}

\section{AG 1: Methodik, Implementierung - Leiter: J. C. Preiß (Berlin), J.} Gensichen (Jena)

S. Conrad (Berlin), A. Timmer (Bremen), P. Layer (Hamburg), M. Böhmig (Frankfurt)

\section{AG 2: Diagnostik - Leiter: K. Herrlinger (Stuttgart),}

T. Kucharzik (Lüneburg)

R. Behrens (Nürnberg, GPGE), C. F. Dietrich (Bad Mergentheim), M. Goetz (Mainz); C. Schmidt (Bonn), A. Schreyer (Regensburg), N. Teich (Leipzig)

AG 3: Pathologie - Leiter: G. B. Baretton (Dresden, DGP),

F. Autschbach (Heilbronn)

D. Aust (Dresden), E. Jehle (Ravensburg), F. Dreher (Rottenburg),

U. Böcker (Mannheim), M. Vieth (Bayreuth)

AG 4: Schub - Leiter: A. Dignass (Frankfurt, DGVS),

E. Stange (Stuttgart)

H. J. Buhr (Berlin, DGVC), J. Stein (Frankfurt), D. Lümmen (Berlin, DCCV), B. Küppers (Mannheim), H. Schulze (Frankfurt), K. M. Keller (Wiesbaden, GPGE)

AG 5: Remissionserhaltung - Leiter: G. Rogler (Zürich),

S. Schreiber (Kiel)

T. Andus (Stuttgart), J. Weismüller (Koblenz), M. Ratzlaff (Reutlingen, DCCV), S. Nikolaus (Kiel), W. Stremmel (Heidelberg), A. Sturm (Berlin), S. Koletzko (München, GPGE)

AG 6: Therapie- und CED-assoziierte Infektionen -

Leiter: A. Stallmach (Jena, DGVS), M. Zeitz (Berlin)

T. Maaser (Lüneburg), B. Siegmund (Berlin), S. Hagel (Jena), H. Köhler (Erlangen, GPGE)

AG 7: Chirurgie und Pouchitis - Leiter: A. J. Kroesen (Köln), S. Fichtner-Feigl (Regensburg)

A. Ballauff (Krefeld, GPGE), K. H. Vestweber (Leverkusen), A. Weimann (Leipzig, DGEM), C. F. Krieglstein (Köln), B. Klump (Stuttgart), C. Witte (Berlin, DGVS), W. Weidlich (Püttlingen, DCCV)

AG 8: Karzinomprophylaxe - Leiter: B. Bokemeyer (Minden),

J. C. Hoffmann (Ludwigshafen, DGVS)

V. Groß (Amberg), B. Kaltz (Bremen, DCCV), R. Porschen (Bremen), C. Pox (Bochum), M. Schleiermacher (Ludwigshafen), J. Mudter (Erlangen)

AG 9: Extraintestinale Manifestationen und CED-assoziierte Erkrankungen - Leiter: M. Reinshagen (Braunschweig), W. Kruis (Kruis)

R. Duchmann (Frankfurt), C. Schmidt (Jena), R. Raedsch (Wiesbaden), S. Buderus (Bonn, GPGE)

AG 10: Psychosomatik, Komplementärmedizin - Leiter: G. Jantschek (Lübeck) ${ }^{1}$, J. Langhorst (Essen)

W. Häuser (Saarbrücken), H. Matthes (Berlin), B. Franzen (Köln), G. Moser (Wien), K. Fellermann (Lübeck)

Die Autoren gedenken Herrn Prof. Dr. Jantschek, der am 12.8.2010 während eines Urlaubs plötzlich und unerwartet verstarb. 


\begin{tabular}{|lll|}
\hline Rubrik & Teilpunkt & Zeitraum \\
\hline Initiierung & Auswahl der Konferenzteilnehmer & $4 / 2009$ \\
& Treffen der AG-Leiter zur Konsentierung der Methodik & 19.4 .2009 \\
\hline Literaturrecherche & Schulung der Literaturbeauftragten & 18.5 .2009 \\
& Definition der Schlüsselempfehlungen & $5-7 / 2009$ \\
& Erstellung von Suchbegriffen für die Literaturrecherche & \\
& Systematische Literaturrecherche & \\
\hline Online-Fragebogenrunde & Erstformulierung der Empfehlungen & $7-10 / 2009$ \\
& AG-Leiter-Treffen & 3.10 .2009 \\
& internetbasierte Beantwortung der Fragebögen durch alle Teilneh- & $11 / 2009$ \\
& mer & \\
\hline Konsuskonferenz & Konsensuskonferenz: Arbeitsgruppensitzungen & 4.12 .2009 \\
& Konsensuskonferenz: Plenarsitzung mit Abstimmung & $4 .-5.12 .2009$ \\
\hline Nachbereitung & Arbeitsgruppenleiter: Überarbeitung der Kapitel & $1-5 / 2010$ \\
& Koordinationsstelle: Zusammenstellen der Leitlinie & $7-12 / 2010$ \\
& Vorlage der Leitlinie bei den unterstützenden Fachgesellschaften & $1 / 2011$ \\
& Veröffentlichung & $9 / 2011$ \\
\hline
\end{tabular}

Tab.2 Zeitplan der Leitlinienerstellung. bei einem Treffen des Koordinationskomitees mit den Arbeitsgruppenleitern konsentiert.

\section{Schlüsselfragen}

Die Leitlinien der DGVS zu den chronisch entzündlichen Darmerkrankungen haben zum Ziel, die gesamte Breite des Krankheitsgebiets abzudecken. Demgegenüber steht ein hoher Aufwand bei der Erstellung hochwertiger klinischer Leitlinien. Daher wurde ein Verfahren zur Priorisierung einzelner Fragestellungen angewandt. Die AG-Leiter definierten aus ihrem jeweiligen Themenbereich Schlüsselfragen, die mit besonderer methodischer Genauigkeit bearbeitet wurden. Die Schlüsselfragen zeichneten sich dadurch aus, dass nach Meinung der AGLeiter diese von vitaler oder hoher klinischer Bedeutung für die Patienten sind, die Antworten besonders strittig sind sowie evtl. von einer besser strukturierten Auswertung der Evidenzen profitieren oder auch zu diesem Gebiet eine Mangelversorgung vorliegt. Für die Schlüsselfragen wurde in besonderem Maße sichergestellt, dass die Literatur, die die Grundlagen für die vorausgegangenen Leitlinien bildete, vollständig erfasst wurde. Darüber hinaus wurde die selektierte Literatur zu den Schlüsselfragen in Evidenztabellen zusammengefasst. Die Schlüsselempfehlungen sind in der Leitlinie durch einen Stern hervorgehoben.

\section{Literatursuche}

Für die systematische Literaturrecherche wurde eine Basissuchstrategie festgelegt, die mit hoher Sensitivität alle systematischen Übersichten, Interventionsstudien und Beobachtungsstudien in MEDLINE zum Thema Colitis ulcerosa bis Mai 2009 erfasst. Diese Suchstrategie wurden dezentral von den Arbeitsgruppen durch für ihr Thema relevante Suchbegriffe ergänzt. Literatur bis 2003 war bereits für die vorausgegangene Leitlinie gesammelt worden, einzig zu den Schlüsselfragen und bisher nicht bearbeiteten Fragen wurde die Literatursuche auf den Zeitraum vor 2003 ausgeweitet. Die Cochrane Library und PsycINFO wurden zentral nach Arbeiten durchsucht, die nicht in MEDLINE erfasst waren. Diese Arbeiten wurden ergänzt durch Literaturstellen, die entweder den AG-Mitgliedern bekannt waren oder in der ECCO-LL bereits zitiert waren. Aus den so identifizierten Arbeiten wurden kontrollierte Studien bzw. deren systematische Übersichten und unkontrollierte

\begin{tabular}{l}
\hline Tab. 3 Konsensusstärken. \\
\begin{tabular}{|l|l|}
\hline starker Konsens & Zustimmung von $>95 \%$ der Teilnehmer \\
\hline Konsens & Zustimmung von $>75-95 \%$ der Teilnehmer \\
\hline $\begin{array}{l}\text { mehrheitliche } \\
\text { Zustimmung }\end{array}$ & Zustimmung von $>50-75 \%$ der Teilnehmer \\
\hline kein Konsens & Zustimmung von weniger als $50 \%$ der Teilnehmer \\
\hline
\end{tabular}
\end{tabular}

Fallserien mit mindestens 20 Patienten eingeschlossen. Die ausgewählte Literatur wurde der Leitliniengruppe zur Konsensuskonferenz in Abstractform bzw. für die Schlüsselempfehlungen als Evidenztabellen zur Verfügung gestellt.

\section{Konsentierung der Empfehlungen}

Die Arbeitsgruppen erstellten zunächst auf dem Boden der DGVS- und der ECCO-Leitlinie unter Berücksichtigung der ausgewählten Literatur Empfehlungen. Auf einem Treffen der AGLeiter am 3.10.2009 wurden Überschneidungspunkte und offene Fragen geklärt, bevor über die Empfehlungen von allen Mitgliedern der Leitliniengruppe in einem Onlineverfahren abgestimmt wurde. Die Ergebnisse und Kommentare der Fragebogenrunde wurden von den AG-Leitern für Umformulierungen der Empfehlungen genutzt. Am 4. und. 5. Dezember 2009 fand eine 2-tägige Konsensuskonferenz statt. Zu Beginn dieser Konferenz wurden die Empfehlungen in den Arbeitsgruppen in Kleingruppen erneut diskutiert und für die Abstimmung im Plenum vorbereitet. Dabei bestand die Möglichkeit für einzelne Mitglieder der Konsensuskonferenz, vorab problematische Formulierungen mit den Mitgliedern der Arbeitsgruppen zu besprechen. Schließlich wurden die Konsensusvorschläge durch jede Arbeitsgruppe vorgetragen, im Plenum diskutiert, ggf. modifiziert und mittels elektronischer, anonymer Abstimmung (TED) verabschiedet. Die Diskussion wurde immer durch einen der nicht in der jeweiligen AG vertretenen Koordinatoren moderiert. Der so erstellte Konsensus wurde durch die Arbeitsgruppenleiter durch Erläuterungen ergänzt. Einzelne Empfehlungen, die in der Fragebogenrunde bereits einen starken Konsens erreicht hatten, die in beiden verwendeten Quellleitlinien gleich lauteten und für die keine neue Literatur identifiziert werden konnten, wurden in der Konsensuskonferenz vorgestellt, aber nicht mehr diskutiert oder abgestimmt. Die Konsensusstärke ( Tab.3) wurde bei jeder Empfehlung angegeben. 
Tab.4 Evidenzklassen.

\begin{tabular}{|c|c|c|c|}
\hline Klasse & Therapie & Diagnostik & Prognose \\
\hline 1a & $\begin{array}{l}\text { systematische Übersicht (SR) }{ }^{1} \text { von randomi- } \\
\text { sierten klinischen Studien (RCTs) }\end{array}$ & $\begin{array}{l}\mathrm{SR}^{1} \text { von diagnostischen Klasse } 1 \text {-Studien; } \\
\mathrm{CDR}^{2} \text { von Klasse } 1 \text { b-Studien aus verschiede- } \\
\text { nen Zentren }\end{array}$ & $\begin{array}{l}\mathrm{SR}^{1} \text { von Inzeptionskohortenstudien; } \mathrm{CDR}^{2} \text {, } \\
\text { validiert in verschiedenen Populationen }\end{array}$ \\
\hline $1 b$ & einzelne RCTs & $\begin{array}{l}\text { Validierungskohortenstudie mit gutem Re- } \\
\text { ferenzstandard; oder } \mathrm{CDR}^{2} \text { getestet in einem } \\
\text { Zentrum }\end{array}$ & $\begin{array}{l}\text { Inzeptionskohortenstudie mit } \geq 80 \% \text { Follow- } \\
\text { up; } \mathrm{CDR}^{2} \text {, validiert in einer Population }\end{array}$ \\
\hline $1 \mathrm{c}$ & Alles-oder-Nichts & Absolute SpPins und SnNouts ${ }^{3}$ & Alles-oder-Nichts-Fallserien \\
\hline $2 a$ & $\mathrm{SR}^{1}$ von Kohortenstudien & $\mathrm{SR}^{1}$ von diagnostischen Klasse 2-Studien & $\begin{array}{l}\mathrm{SR}^{1} \text { von retrospektiven Kohortenstudien } \\
\text { oder Placebo-Gruppen in RCTs }\end{array}$ \\
\hline $2 b$ & $\begin{array}{l}\text { Einzelne Kohortenstudie oder RCTs minderer } \\
\text { Qualität }\end{array}$ & $\begin{array}{l}\text { explorative Kohortenstudien mit gutem Re- } \\
\text { ferenzstandard; CDR }{ }^{2} \text { nach Ableitung oder } \\
\text { validiert nur an Teilgruppen oder Datenban- } \\
\text { ken }\end{array}$ & $\begin{array}{l}\text { retrospektive Kohortenstudie oder Follow- } \\
\text { up der Placebogruppe in einem RCT; CDR } \\
\text { nach Ableitung oder validiert an Teilgruppen }\end{array}$ \\
\hline $2 c$ & Outcomes-Studien, Ökologische Studien & & Outcomes-Studien \\
\hline $3 a$ & $\mathrm{SR}^{1}$ von Fallkontrollstudien & $\mathrm{SR}^{1}$ von Klasse 3-Studien & \\
\hline $3 b$ & einzelne Fallkontrollstudie & $\begin{array}{l}\text { nicht konsekutive Studie; oder ohne konsis- } \\
\text { tent angewandten Referenzstandard }\end{array}$ & \\
\hline 4 & $\begin{array}{l}\text { (Fallserien oder) Kohorten-/Fall-Kontroll- } \\
\text { studien minderer Qualität }\end{array}$ & $\begin{array}{l}\text { Fallkontrollstudien, schlechter oder nicht } \\
\text { unabhängiger Referenzstandard }\end{array}$ & $\begin{array}{l}\text { Fallserien oder prognostische Kohortenstu- } \\
\text { dien mäßiger Qualität }\end{array}$ \\
\hline 5 & $\begin{array}{l}\text { Expertenmeinung ohne explizite Bewertung } \\
\text { der Evdenz oder basierend auf physiologi- } \\
\text { schen Modellen, Laborforschung oder Defi- } \\
\text { nitionen }\end{array}$ & $\begin{array}{l}\text { Expertenmeinung ohne explizite Bewertung } \\
\text { der Evidenz oder basierend auf physiologi- } \\
\text { schen Modellen, Laborforschung oder Defi- } \\
\text { nitionen }\end{array}$ & $\begin{array}{l}\text { Expertenmeinung ohne explizite Bewertung } \\
\text { der Evdenz oder basierend auf physiologi- } \\
\text { schen Modellen, Laborforschung oder Defi- } \\
\text { nitionen }\end{array}$ \\
\hline
\end{tabular}

1 Systemischer Review mit Homogenität.

${ }^{2}$ Clinical Decision Rule (Algorithmen oder Punktesysteme, die helfen, eine Prognose oder diagnostische Kategorie abzuschätzen).

${ }^{3}$ SpPins haben eine so hohe Spezifität, dass sie die definitive Diagnose stellen, SnNouts haben eine so hohe Sensitivität, dass ein negatives Ergebnis die Diagnose ausschließt.

\section{Tab.5 Evidenzgrade. \\ A Direkt anwendbare Studien der Evidenzklasse 1 \\ B Studien der Evidenzklasse 2 oder 3 oder indirekte Anwendbarkeit ${ }^{1}$ von Studien der Evidenzklasse 1 \\ C Studien der Evidenzklasse 4 oder indirekte Anwendbarkeit ${ }^{1}$ von Studien der Evidenzklasse 2 oder 3 \\ D Studien der Evidenzklasse 5 oder indirekte Anwendbarkeit ${ }^{1}$ von Studien der Evidenzklasse 4 oder beunruhigend uneinheitliche oder nicht aussagekräftige Stu- dien irgendeiner Evidenzklasse \\ 1 Indirekte Anwendbarkeit bezieht sich auf Situationen, die sich möglicher- weise klinisch relevant von der in der vorliegenden Evidenz untersuchten Situation unterscheiden (z. B. unterschiedliche Klasse von Antidepressiva oder die Studie war an unterschiedlichen Populationen durchgeführt wor- den).}

\section{Klassifikation der Evidenzklassen, Anwendbarkeit, Konsens, Evidenzgrade}

In manchen Leitlinien ergibt sich die Stärke der Empfehlung automatisch aus der Evidenzklasse („Level of Evidence“). Dies führt dazu, dass Medikamente, die in großen Studien untersucht wurden, grundsätzlich eine stärkere Empfehlung erhalten als Medikamente, die möglicherweise wirksamer sind, aber nicht so gut untersucht wurden. Um diesem Dilemma zu entgehen, muss ein solcher Automatismus vermieden werden. Entsprechend dem Regelwerk der AWMF, den methodischen Empfehlungen der GRADE Working Group [7] und einigen der neueren DGVS-Leitlinien haben wir eine Trennung von Evidenzklasse und Empfehlungsstärke übernommen. Die Evidenzklasse und der Evidenzgrad („Grade of Recommendation“, nicht: Empfehlungsstärke) wurden hier entsprechend des Oxford-Centre for Evidence Based Medicine eingeteilt $(\bullet$ Tab. 4, 5) und basiert auf dem Stu- diendesign und der direkten oder indirekten Anwendbarkeit [8]. Der Evidenzgrad gibt das Vertrauen in die zugrunde liegende Evidenz wieder.

Die Empfehlungsstärke wiederum wurde festgelegt in Abhängigkeit von potenziellem Nutzen und dem Risiko der Intervention, dem Evidenzgrad, den Patientenpräferenzen, der Umsetzbarkeit und mitunter auch ökonomischen Überlegungen. Die Empfehlungsstärke spiegelt sich v.a. in der Formulierung der Empfehlung wider und wurde wie in den Nationalen Versorgungsleitlinien eingeteilt ( $\bullet$ Tab.6). Empfehlungssgrad und der Empfehlungsstärke sind in der gedruckten Leitlinie zusammen mit der Konsensusstärke angegeben.

\section{Kapitel 2: Klinische Diagnostik \\ $\nabla$}

\section{Klassifikation}

\section{Empfehlung 2.1*}

Eine Klassifikation bez. der Ausdehnung der Erkrankung soll erfolgen.

Evidenzgrad: D

Konsensusstärke: starker Konsens

Empfehlungsstärke: $\uparrow \uparrow$

\section{Empfehlung 2.2*}

Es soll eine endoskopische Einteilung in die Proktitis (begrenzt auf das Rektum), die Linksseitenkolitis (Ausdehnung bis zur linken Flexur) und die ausgedehnte Kolitis erfolgen.

Evidenzgrad: D

Konsensusstärke: starker Konsens

Empfehlungsstärke: $\uparrow \uparrow$ 
Tab. 6 Empfehlungsstärken.

\begin{tabular}{|c|c|c|c|c|}
\hline Empfehlungsstärke & Formulierung & Bedeutung für Ärzte & Bedeutung für Patienten & Symbol \\
\hline stark positiv & „soll“ & $\begin{array}{l}\text { Die meisten Patienten sollen die empfohlene Intervention } \\
\text { erhalten } \\
\text { „Definitely do it!“ }\end{array}$ & $\begin{array}{l}\text { Die meisten Patienten würden sich für die } \\
\text { empfohlene Intervention entscheiden und } \\
\text { nur eine kleine Zahl dagegen }\end{array}$ & $\uparrow \uparrow$ \\
\hline $\begin{array}{l}\text { abgeschwächt } \\
\text { positiv }\end{array}$ & $\begin{array}{l}\text { "sollte“ } \\
\text { oder } \\
\text { "kann" }\end{array}$ & $\begin{array}{l}\text { Unterschiedliche Entscheidungen sind bei verschiedenen } \\
\text { Patienten angemessen, die von der Situation des Patienten } \\
\text { abhängen, aber auch von seinen persönlichen Vorstellungen } \\
\text { und Präferenzen } \\
\text { „Probably do it!“ }\end{array}$ & $\begin{array}{l}\text { Die Mehrzahl der Patienten würde sich für die } \\
\text { Intervention entscheiden, aber viele nicht }\end{array}$ & $\uparrow$ \\
\hline $\begin{array}{l}\text { abgeschwächt } \\
\text { negativ }\end{array}$ & $\begin{array}{l}\text { "sollte eher } \\
\text { nicht" }\end{array}$ & „Probably don’t do it!“ & $\begin{array}{l}\text { Die Mehrzahl der Patienten würde sich gegen } \\
\text { die Intervention entscheiden, aber viele auch } \\
\text { dafür }\end{array}$ & $\downarrow$ \\
\hline stark negativ & "soll nicht“ & „Definitely don’t do it!“ & $\begin{array}{l}\text { Die meisten Patienten würden sich gegen die } \\
\text { empfohlene Intervention entscheiden und } \\
\text { nur eine kleine Zahl dafür }\end{array}$ & $\downarrow \downarrow$ \\
\hline unklar & "5 & fehlung bezüglich ... kan & atenlage nicht gegeben werden." & $\leftarrow$ \\
\hline
\end{tabular}

Tab.7 Ausdehnung der Colitis ulcerosa (nach Silverberg et al. [13])

\begin{tabular}{|c|c|c|}
\hline Einteilung & Ausdehnung & Beschreibung \\
\hline E1 & Proktitis & $\begin{array}{l}\text { limitiert auf das Rektum (distal des } \\
\text { rektosigmoidalen Übergangs) }\end{array}$ \\
\hline E2 & Linksseitenkolitis & Befall bis zur linken Flexur \\
\hline E3 & $\begin{array}{l}\text { ausgedehnte Ko- } \\
\text { litis }\end{array}$ & $\begin{array}{l}\text { Ausdehnung über die linke Flexur } \\
\text { hinaus bis zur Pankolitis }\end{array}$ \\
\hline
\end{tabular}

\section{Erläuterung}

Eine Klassifikation der Colitis ulcerosa nach der Ausdehnung der Erkrankung ist aus zwei Gründen sinnvoll. Einerseits entscheidet die Lokalisation der Erkrankung über den Einsatz topisch und/oder systemisch wirkender Medikamente, insbesondere der 5-ASA-Präparate. Bei einer Proktitis werden bevorzugt Suppositorien eingesetzt, bei der Linksseitenkolitis können Einläufe oder Schaumpräparate zum Einsatz kommen. Bei ausgedehnter Colitis ulcerosa sollten oral verabreichte Substanzen verwendet werden, einer aktuellen Studie zufolge allerdings idealerweise kombiniert mit einer topischen Therapie [12]. Weiterhin hat die Ausdehnung Einfluss auf den Beginn des Karzinomüberwachungsprogramms. Aus diesem Grund wird die Empfehlung zur Vorsorgekoloskopie entsprechend der Ausdehnung der Erkrankung differenziert ausgesprochen (siehe Kapitel 8). Die bevorzugte Einteilung der Colitis ulcerosa unterscheidet die Proktitis, die Linksseitenkolitis sowie die ausgedehnte Kolitis mit Ausdehnung über die linke Flexur hinaus (Montreal-Klassifikation) [13] ( Tab.7). Wegen der großen Bedeutung, die diese Empfehlung für eine Vielzahl von Therapieentscheidungen bei der Colitis ulcerosa hat, wird hier trotz schlechter Evidenz eine starke Empfehlung abgegeben.

\section{Empfehlung 2.3}

Das gleichzeitige Vorliegen einer PSC soll dokumentiert werden, da dies die Überwachungsstrategie beeinflusst.

[Evidenzgrad: C, Konsensusstärke: starker Konsens, Empfehlungsstärke: $\uparrow \uparrow]$

\section{Erläuterung}

Die Dokumentation einer primär sklerosierenden Cholangitis (PSC) ist von Bedeutung, da ihr Vorliegen mit einem deutlich er- höhten Risiko für die Entwicklung eines kolorektalen Karzinoms vergesellschaftet ist $[14,15]$. Diese Tatsache hat Einfluss auf die Empfehlungen zur Karzinomüberwachung bei gleichzeitigem Vorliegen von Colitis ulcerosa und PSC (siehe Kapitel 8).

\section{Krankengeschichte}

\section{Empfehlung 2.4}

Die Anamnese soll eine detaillierte Erfragung über Art und Beginn der Symptome, eine kürzliche Reiseanamnese, Nahrungsmittelunverträglichkeiten, Kontakte mit infektiösen Durchfallerkrankungen und die Medikamentenanamnese (insbesondere bez. Antibiotika und nicht steroidaler Antirheumatika) beeinhalten.

[Evidenzgrad: D, Konsensusstärke: starker Konsens, Empfehlungsstärke: $\uparrow \uparrow$ ]

\section{Empfehlung 2.5}

Bei etablierter Diagnose sollte die Anamnese weiterhin den Impfstatus, die Raucheranamnese, die Familien- und Sozialanamnese und die Frage nach einer evtl. vorliegenden Depression beinhalten.

[Evidenzgrad: D, Konsensusstärke: starker Konsens, Empfehlungsstärke: $\uparrow$ ]

\section{Erläuterung}

Eine ausführliche Anamnese und körperliche Untersuchung gehört zur täglichen Routine und ist nicht spezifisch für Patienten mit Colitis ulcerosa. Nichtsdestotrotz gibt es bestimmte Aspekte, die bei der Colitis ulcerosa (bzw. den chronisch entzündlichen Darmerkrankungen) eine besondere Rolle spielen und eine besondere Aufmerksamkeit verdienen. Daher wird hier im Sinne eines „good practice points“ eine starke Empfehlung abgegeben.

Die Unterscheidung zwischen Morbus Crohn und Colitis ulcerosa kann manchmal schwierig bzw. anamnestisch unmöglich sein. Das Fehlen von rektalem Blutabgang oder Symptome bei aktiven Rauchern sollten den Verdacht auf einen Morbus Crohn erwecken. Eine infektiöse oder medikamenteninduzierte Colitis sollte anamnestisch, soweit möglich, abgegrenzt werden. Die Einnahme von nicht steroidalen Antirheumatika (NSAR) scheint ein signifikantes Risiko zu beeinhalten, eine bestehende Colitis ulcerosa zu verschlechtern [16-19]. 
Knapp die Hälfte der Patienten mit Colitis ulcerosa erlebt einen Verlauf der Erkrankung, der eine immunsuppressive Therapie mit Steroiden, Thiopurinen oder anti-TNF-Antikörpern erfordert [20, 21]. Das Risiko für opportunistische Infektionen unter immunsuppressiver Therapie, insbesondere unter Mehrfachimmunsuppression, ist signifikant erhöht [22]. Daher wird sowohl in einem Konsensuspapier der ECCO als auch im vorliegenden Konsensuspapier der DGVS die Erhebung und ggf. Vervollständigung des Impfstatus gefordert (für Details siehe Kapitel 6).

Aktives Rauchen hat einen protektiven Effekt auf die Entwicklung und den Schweregrad einer Colitis ulcerosa [23, 24]. ExRaucher hingegen haben ein 70\% höheres Risiko, eine Colitis ulcerosa zu entwickeln, diese weist häufiger einen refraktären und ausgedehnten Verlauf auf, auch im Vergleich zu Patienten, die nie geraucht haben. Krankenhausaufenthalte und Kolektomieraten sind bei Ex-Rauchern ebenfalls höher als bei Patienten, die nie geraucht haben $[25,26]$. Ex-Raucher, die wieder beginnen, zu rauchen, scheinen einen milderen Verlauf der Erkrankung zu erleben $[26,27]$. Umstritten ist die Frage, ob Rauchen auch das Auftreten einer PSC oder das Auftreten einer Pouchitis nach Kolektomie und ileo-analer Pouchanlage verhindern kann [28-30]. Ein weiterer Zusammenhang scheint zwischen Appendektomie und der Entwicklung einer Colitis ulcerosa zu bestehen. Kohortenstudien und eine Metaanalyse weisen daraufhin, dass eine Appendektomie in der Kindheit (wegen „echter“ Appendizitis) einen protektiven Effekt (69\% Risikoreduktion) bzgl. des Auftretens und des Schweregrads einer späteren Colitis ulcerosa hat. Auch diese Daten sind nicht in allen Folgestudien bestätigt worden [31-37]. Dabei scheint der protektive Effekt der Appendektomie zusätzlich zum Effekt des Rauchens zu bestehen, während die Appendektomie nicht vor der Entwicklung einer PSC schützt. Eine Appendektomie nach dem Auftreten einer Colitis ulcerosa scheint keinen wesentlichen Effekt mehr zu haben. Die Daten zur Appendektomie sind epidemiologisch und pathophysiologisch interessant, haben im klinischen Alltag allerdings keine therapeutische Konsequenz und werden daher in den Empfehlungen nicht berücksichtigt.

Die Familienanamnese hat einen besonderen Stellenwert in der Anamnese. Erstgradige Verwandte von Patienten mit Colitis ulcerosa haben ein 10- bis 15-fach erhöhtes Risiko, ebenfalls an einer Colitis ulcerosa zu erkranken [38]. Allerdings beträgt das lebenslange Risiko für erstgradige Verwandte, eine Colitis ulcerosa zu entwickeln, nur ungefähr 5\% (oder umgekehrt liegt die Wahrscheinlichkeit, nicht nicht an Colitis ulcerosa zu erkranken, bei 95\%). Dieses ist eine wichtige Information für Patienten bei der Familienplanung. Familiäre Fälle von Colitis ulcerosa scheinen eher das weibliche Geschlecht zu betreffen, weiterhin scheint das Erkrankungsalter niedriger zu liegen im Vergleich zu sporadischen Fällen [39].

\section{Empfehlung 2.6}

Weiterhin soll die Anamnese Fragen bzgl. extraintestinaler Manifestationen (Mund, Haut, Augen und/oder Gelenke) sowie nach perianalen Abszessen, Fisteln und Analfissuren beinhalten.

[Evidenzgrad: D, Konsensusstärke: starker Konsens, Empfehlungsstärke: $\uparrow \uparrow$ ]

\section{Empfehlung 2.7}

Bei Erstdiagnose und beim Auftreten spezifischer Symptome soll eine komplette körperliche Untersuchung inkl. einer oralen und perianalen Inspektion, einer rektalen Untersuchung und die Beachtung evtl. vorliegender extraintestinaler Manifestationen erfolgen.

[Evidenzgrad: D, Konsensusstärke: Konsens, Empfehlungsstärke: $\uparrow \uparrow$ ]

\section{Erläuterung}

Explizit sollte nach extraintestinalen Manifestationen, insbesondere an Augen, Mund, Gelenken und der Haut sowie nach perianalen Manifestationen, gefragt werden, da diese vom Patienten mitunter nicht mit der Krankheit in Zusammenhang gebracht werden, aber von hoher Bedeutung für die weiteren therapeutischen Entscheidungen sein können [40-44]. Die körperliche Untersuchung ist bei Patienten mit Colitis ulcerosa in Abwesenheit extraintestinaler Manifestationen meist wenig spezifisch. Die klinische Symptomatik insbesondere mit Durchfällen, Tenesmen und rektalem Blutabgang ist häufig wegweisend. Bei schwerem Schub können Tachykardie, Gewichtsverlust, abdominelle Abwehrspannung und/oder abgeschwächte Darmgeräusche auftreten. In der Arbeitsgruppe und auch im Plenum wurde die Notwendigkeit einer perinealen Inspektion sowie der rektalen Untersuchung intensiviert diskutiert. Selbstverständlich ist sie nicht bei jeder Vorstellung des Patienten notwendig, zudem wird sie häufig regelhaft im Rahmen einer Koloskopie durchgeführt. Allerdings soll hier nochmals auf das erhöhte Risiko (kolo-)rektaler Karzinome bei Colitis ulcerosa hingewiesen werden, sodass die explizite Dokumentation der rektalen Untersuchung gerechtfertigt scheint.

\section{Empfehlung 2.8}

Bei Kindern und Jugendlichen soll zusätzlich die Entwicklung von Gewicht, Länge und das Pubertätsstadium bei Erstdiagnose und regelmäßig im Krankheitsverlauf erfasst werden.

[Evidenzgrad: D, Konsensusstärke: Konsens, Empfehlungsstärke: $\uparrow \uparrow]$

\section{Erläuterung}

Bei Kindern und Jugendlichen ist zur Diagnose von Wachstumsverzögerungen bei jeder Visite das Längensollgewicht oder der BMI im Vergleich zu nationalen geschlechtsspezifischen Referenzwerten zu erheben. Bei Wachstumsretardierung ist durch eine Röntgenaufnahme der linken Hand das Knochenalter zu bestimmen (internationale Vereinbarung: links). Weiterhin sollte bis zum Abschluss der Pubertät 1- bis 2-mal pro Jahr das Pubertätsstadium nach Tanner bestimmt werden [45]. Die Leitliniengruppe geht davon aus, dass eine Abweichung von dieser Empfehlung mit bleibenden Konsequenzen für die Patienten verbunden sein kann, sodass hier eine starke Empfehlung abgegeben wurde.

\section{Empfehlung 2.9}

Die Diagnose einer Colitis ulcerosa soll auf dem Boden einer Kombination von Anamnese, klinischer Untersuchung und typischen laborchemischen, sonografischen, endoskopischen und histologischen Befunden gestellt werden.

[Evidenzgrad: D, Konsensusstärke: starker Konsens, Empfehlungsstärke: $\uparrow \uparrow$ ] 


\section{Empfehlung 2.10}

Bei Zweifel bzgl. der Diagnose soll die Endoskopie inkl. Histologiegewinnung im Intervall wiederholt werden.

[Evidenzgrad: D, Konsensusstärke: Konsens, Empfehlungsstärke: $\uparrow \uparrow]$

\section{Erläuterung}

Der natürliche Verlauf der Colitis ulcerosa ist charakterisiert durch Episoden von Krankheitsschüben, die sich mit Phasen der Remission abwechseln. Selten (nur ca. 5\% der Patienten) kann der Krankheitsverlauf auch kontinuierlich ohne intermittierende Remissionsphasen verlaufen. Dies gilt insbesondere für pädiatrische Patienten. Ebenso häufig kann sich die Colitis ulcerosa auch als einzelner Schub mit anschließender prolongierter Remission präsentieren [46]. Eine zügige Etablierung der Diagnose inklusive Ausdehnung und Schweregrad des Schubes ermöglicht eine optimale Therapiestrategie. Ein Goldstandard für die Diagnosestellung der Colitis ulcerosa existiert allerdings nicht; die Diagnose beruht auf der Kombination typischer Befunde in der Anamnese, der Endoskopie, der Sonografie, radiologischer Techniken und der Histopathologie. Die pathomorphologischen Kriterien ergeben sich aus der Biopsieentnahme bei der Endoskopie oder aus der Aufarbeitung von Operationspräparaten. Normalbefunde in der Histopathologie der Schleimhautbiopsien schließen eine aktive Colitis ulcerosa aus. Nach Etablierung der Diagnose ändert sich bei ca. 10\% der Patienten innerhalb der ersten 5 Jahre nach Diagnosestellung die Diagnose zu einem Morbus Crohn, oder die Diagnose einer chronisch entzündlichen Darmerkrankung wird insgesamt verworfen. Daher ist insbesondere bei Zweifeln an der Diagnose die endoskopische und histopathologische Bestätigung durch eine erneute Endoskopie mit Histologiegewinnung geboten [47]. Bei einer Minderheit der Patienten wird es auch im Verlauf nicht möglich sein, die exakte Zuordnung zu den Entitäten Colitis ulcerosa oder Morbus Crohn vorzunehmen, hier spricht man von der sog. unklassifizierbaren Kolitis bzw. einer „Colitis indeterminata“ $[13,48]$.

\section{Diagnosestellung}

\section{Empfehlung 2.11}

Die initiale Labordiagnostik soll neben dem Blutbild mindestens folgende Parameter enthalten: Entzündungsstatus, Eisenhaushalt, Nierenfunktion, Transaminasen und Cholestaseparameter.

[Evidenzgrad: D, Konsensusstärke: starker Konsens, Empfehlungsstärke: $\uparrow \uparrow]$

\section{Empfehlung 2.12}

Für die begleitende laborchemische Diagnostik eines Ansprechens auf die Therapie können CRP, BSG, Blutbild sowie fäkale Neutrophilenmarker herangezogen werden.

[Evidenzgrad: B, Konsensusstärke: starker Konsens, Empfehlungsstärke: $\uparrow$ ]

\section{Erläuterung}

Bei jedem Patienten mit aktiver Colitis ulcerosa sollte als minimale Labordiagnostik ein Blutbild, inflammatorische Marker (CRP oder BSG), Parameter des Eisenhaushalts, Nierenreten- tionsparameter, Transaminasen und Cholestaseparameter erhoben werden. Dabei können die Laborwerte, insbesondere bei milder bis moderater Colitis ulcerosa und/oder distalem Befallsmuster, durchaus normal ausfallen. Ausgenommen bei der Proktitis, bei der in der Regel keine auffälligen Laborwerte zu erwarten sind, korreliert das C-reaktive Protein mit der Ausdehnung der Erkrankung sowie schwach mit der klinischen Aktivität [49, 50]. Grundsätzlich fällt der CRP-Anstieg bei Patienten mit CU in der Regel geringer aus als bei Patienten mit Morbus Crohn, kann jedoch bei CRP-positiven Patienten durchaus auch als Marker für die klinische und endoskopische Krankheitsaktivität herangezogen werden [51]. Bei Patienten mit schwerer Krankheitsaktivität ist in der Regel auch die BSG erhöht und es besteht eine Anämie.

Allerdings können weder das CRP noch die BSG naturgemäß die Colitis ulcerosa von einer infektiösen Ursache differenzieren. Zwei kleine Studien berichten über einen Stellenwert von Procalcitonin zur Abgrenzung selbstlimitierender Kolitiden [52, 53]. Insbesondere bei der Erstdiagnostik sind zur Abgrenzung und zur Diagnostik von selbstlimitierenden infektiösen Kolitiden Stuhlkulturen hilfreich [54] (siehe Empfehlung 2.13 und 2.14). Bei pädiatrischen Patienten sollten bei Verdacht auf eine Colitis ulcerosa ein großes Blutbild, CRP (alternativ auch die BSG) und Kreatinin sowie Albumin und Leberparameter (GOT, GPT und g-GT) bestimmt werden [55]. Bezüglich der notwendigen Laborkontrollen unter immunsuppressiver Therapie wird auf die entsprechenden Therapiekapitel verwiesen. Die Wertigkeit fäkaler Stuhlmarker und insbesondere des Calprotectins als Marker für die klinische und endoskopische Entzündungsaktivität bei Colitis ulcerosa konnte in mehreren Studien herausgestellt werden [56, 57].

Die routinemäßige Bestimmung von perinukleären anti-neutrophilen cytoplasmatischen Antikörpern (pANCA) für die Colitis ulcerosa und anti-Saccharomyces cerevisiae Antikörpern (ASCA) für den Morbus Crohn zur Diffentierung der beiden Krankheitsentitäten wird wegen der niedrigen Sensitivität nicht empfohlen [58], kann allerdings im Einzelfall hilfreich sein. In den meisten Publikationen werden pANCA bei maximal 65\% der Patienten mit Colitis ulcerosa und in weniger als $10 \%$ bei Patienten mit Morbus Crohn beschrieben [59, 60]. Eine große Metaanalyse mit Berücksichtigung von 60 Studien konnte zeigen, dass die Sensitivität für die Detektion eines Morbus Crohn bei der Konstellation ASCA+/pANCA- bei 55\% liegt (Spezifität 93\%), während die Sensitivität für die Detektion einer Colitis ulcerosa bei der Konstellation pANCA + /ASCAbei $51 \%$ (Spezifität $94 \%$ ) und bei pädiatrischen Patienten sogar noch besser liegt (70 bzw. 93\%) [61].

\section{Empfehlung 2.13}

Eine intestinale Infektion soll ausgeschlossen werden.

[Evidenzgrad: D, Konsensusstärke: Konsens, Empfehlungsstärke: $\uparrow \uparrow]$

\section{Empfehlung 2.14}

Bei der Erstdiagnostik soll eine mikrobiologische Diagnostik auf bakterielle infektiöse Erreger inklusive Clostridium-difficile-Toxin erfolgen.

[Evidenzgrad: B, Konsensusstärke: starker Konsens, Empfehlungsstärke: $\uparrow \uparrow$ ] 


\section{Empfehlung 2.15}

Bei Patienten mit entsprechender Reiseanamnese soll eine ergänzende Diagnostik bzgl. landestypischer Erreger durchgeführt werden.

[Evidenzgrad: D, Konsensusstärke: starker Konsens, Empfehlungsstärke: $\uparrow \uparrow]$

\section{Erläuterung}

In der initialen Diagnostik ist die Abgrenzung gegenüber infektiösen, in der Regel selbst limitierten Ursachen wichtig. Die sorgfältige Differenzialdiagnostik wird von der Leitliniengruppe als „good practice point“ angesehen. Stuhlproben sollten untersucht werden auf die häufigen Erreger inklusive Campylobacter spp. und Clostridium difficile Toxin A and B. Bei entsprechender Anamnese können spezielle Stuhluntersuchungen sinnvoll sein wie die Stuhlmikroskopie und Stuhlantigendiagnostik auf Amöben oder andere Parasiten.

\section{Empfehlung 2.16*}

Bei etablierter Colitis ulcerosa soll bei schwerem Schub und bei therapierefraktärem Verlauf bzw. vor Intensivierung einer immunsuppressiven Therapie eine mikrobiologische Diagnostik inklusive Untersuchungen auf Clostridium-difficile-Toxin und Cytomegalievirus erfolgen.

[Evidenzgrad: B, Konsensusstärke: starker Konsens, Empfehlungsstärke: $\uparrow \uparrow]$

\section{Empfehlung 2.17}

Die Diagnostik bzgl. Clostridium difficile soll mittels Toxinnachweis im Stuhl, bei therapierefraktärem Krankheitsverlauf und negativem Toxinnachweis zusätzlich durch Endoskopie mit Biopsie erfolgen.

[Evidenzgrad: C, Konsensusstärke: starker Konsens, Empfehlungsstärke: $\uparrow \uparrow]$

\section{Empfehlung 2.18}

Die CMV-Diagnostik soll eine CMV-PCR aus dem Blut oder die Histologie einschließlich einer Immunhistochemie aus der Darmbiopsie beinhalten.

[Evidenzgrad: D, Konsensusstärke: starker Konsens, Empfehlungsstärke: $\uparrow \uparrow]$

\section{Erläuterung}

Im weiteren Verlauf muss nicht bei jedem Schub die Stuhldiagnostik wiederholt werden [62-64]; allerdings sollte insbesondere die Diagnostik auf C. difficile und Cytomegalievirus (CMV)-(Re-)Infektionen bei schweren oder refraktären Verläufen erfolgen oder falls anamnestisch dem Schub eine Einnahme von Antibiotika vorausgegangen ist $[65,66]$. Hinzuweisen ist auch auf die Tatsache, dass ein negativer Stuhltest auf C.difficile-Toxine nicht ausreicht, um eine pseudomembranöse Kolitis auszuschließen. Bei CU-Patienten reichen wohl niedrigere Toxinmengen (die der Diagnostik entgehen) aus, um eine pseudomembranöse Kolitis auszulösen. Wiederholte Stuhl-Untersuchungen zum Toxinnachweis oder die Durchführung einer C.-difficile-Kultur mit anschließendem Toxinnachweis werden deshalb empfohlen. In diesen Fällen kann auch eine Sigmoidoskopie weitere Informationen bringen, insbesondere bei refraktären Patienten mit negativen Stuhlkulturen [67].

Die Reaktivierung einer latenten CMV-Infektion tritt insbesondere - jedoch nicht ausschließlich - bei immunsupprimierten Patienten (systemische Steroide, Azathioprin, Calcineurininhibitoren) mit schwerer Kolitis auf (52-79\%) [68-70]. Grundsätzlich kann eine CMV-Infektion ein refraktäres bzw. schweres Rezidiv auslösen, wenngleich die klinische Relevanz des Nachweises unsicher ist. Die optimale Methode zur Detektion einer klinisch relevanten CMV-Infektion bei Patienten mit Kolitis ist bisher nicht etabliert. Der gelegentliche Nachweis intranukleärer Einschlusskörper im Rahmen einer histopathologischen Untersuchung zeigt nicht notwendigerweise eine klinisch signifikante CMV-Infektion an. Hingegen sind multiple intranukleäre Einschlusskörperchen häufig als beweisend anzusehen [71, 72] (bzgl. der Therapieindikation und -modalitäten siehe hierzu Kapitel 6).

\section{Empfehlung 2.19}

Die quantitative Bestimmung von fäkalen Neutrophilenmarkern im Stuhl kann zur Abgrenzung nicht entzündlicher Ursachen der gastrointestinalen Beschwerden genutzt werden.

[Evidenzgrad: B, Konsensusstärke: starker Konsens, Empfehlungsstärke: $\uparrow$ ]

\section{Erläuterung}

Verschiedene fäkale Entzündungsmarker wie Calprotectin, Lysozym, PMN-Elastase, Lactoferrin und S100 A12 sind bei chronisch entzündlichen Darmerkrankungen untersucht worden [73-75]. Calprotectin und Lactoferrin-Konzentrationen besitzen wohl die höchste Sensitivität für eine intestinale Entzündung und korrelieren mit dem klinischen und endoskopischen Entzündungsgrad der Colitis ulcerosa [56, 57, 76-80]. S100 A12 ist ein neuer fäkaler Marker, der ebenfalls ein hochsensitiver Parameter für die Entzündungsaktivität und das Therapieansprechen bei Colitis ulcerosa zu sein scheint, die Datenlage hierfür ist jedoch noch gering [81-84]. Fäkale Marker sind nicht in der Lage, zwischen verschiedenen Ursachen einer intestinalen Entzündung zu unterscheiden. Aus diesem Grund ist der diagnostische Nutzen in der Primärdiagnostik der Colitis ulcerosa limitiert. Allerdings können fäkale Marker zur Abgrenzung funktioneller Beschwerden und insbesondere in der pädiatrischen Diagnostik hilfreich sein [85-87]. In mehreren Untersuchungen konnte belegt werden, dass sowohl pädiatrische als auch erwachsene Patienten mit chronisch-entzündlichen Darmerkrankungen signifikant höhere fäkale Calprotectinwerte aufweisen als die Normalbevölkerung und als Patienten mit Reizdarmsyndrom. Die Marker können daher sehr gut als Differenzierungsmarker zum Reizdarmsyndrom eingesetzt werden [82].

Darüber hinaus konnte in mehreren Studien gezeigt werden, dass u.a. Calprotectin als Marker für die Früherkennung von Rezidiven bei Colitis ulcerosa herangezogen werden kann, da endoskopische Rezidive mit hoher Sensitivität und Spezifität vor Auftreten einer klinischer Symptomatik erkannt werden $[78,88,89]$. 


\section{Endoskopische Diagnostik}

\section{Empfehlung 2.20}

Bei Verdacht auf Colitis ulcerosa soll eine Ileokoloskopie mit segmentalen Biopsien aus allen Darmabschnitten erfolgen, um die Diagnose zu stellen und die Ausdehnung der Erkrankung festzustellen (siehe hierzu auch Kapitel 3).

[Evidenzgrad: D, Konsensusstärke: Konsens, Empfehlungsstärke: $\uparrow \uparrow]$

Die komplette Koloskopie mit Intubation des terminalen Ileums und segmentaler Entnahme von Darmbiopsien wird bei Patienten mit Verdacht auf Colitis ulcerosa in der Erstdiagnostik einer Sigmoidoskopie vorgezogen, um einerseits das Befallsmuster klassifizieren zu können und andererseits eine Ileitis terminalis Crohn weitgehend auszuschließen [90, 91]. Im Verlauf kann bei Patienten mit akuter schwerer Colitis zur Abschätzung der Krankheitsaktivität in der Regel auf eine komplette Koloskopie sowie eine entsprechende Darmvorbereitung verzichtet werden und nur eine Sigmoidoskopie durchgeführt werden. Mithilfe einer Koloskopie kann in der Mehrzahl der Fälle die Diagnose sowie die Ausdehnung der Erkrankung festgelegt werden. Dieses Vorgehen erscheint kosteneffektiver zu sein als eine Indexsigmoidoskopie, wie sie von einer kleinen Zahl der Konsensuskonferenz-Teilnehmer favorisiert wurde $[92,93]$. In der Diskussion bestand jedoch Einigkeit über die Stärke der Empfehlung.

Die Koloskopie bei pädiatrischen Patienten sollte in Intubationsnarkose oder zumindest tiefer Analgosedierung erfolgen, wobei die Durchführung eine entsprechende Strukturqualität bezogen auf die Reanimationsmöglichkeit von pädiatrischen Patienten voraussetzt.

Die virtuelle Kolonografie ist eine sich entwickelnde Technologie, die bisher keine diagnostische Wertigkeit bez. der Festlegung der Krankheitsausdehnung bei Patienten mit Colitis ulcerosa hat. Die Wertigkeit der MR-Kolonografie und der CTKolonografie bei Colitis ulcerosa wurde bisher nur in wenigen Studien mit einer geringen Anzahl von Patienten untersucht. Die Ergebnisse sind z.T. widersprüchlich, geringgradige Veränderungen in der Mukosa wie Erosionen oder flache Polypen können mit diesen Methoden nur unvollständig dargestellt werden, sodass sich diese Methode in der Diagnostik der Colitis ulcerosa zur Zeit keinen Stellenwert hat [55, 94-96].

\section{Empfehlung 2.21}

Eine routinemäßige Koloskopie soll bei Patienten mit Colitis ulcerosa in der Remission bis zum Beginn der Karzinomüberwachung nicht erfolgen.

[Evidenzgrad: D, Konsensusstärke: starker Konsens, Empfehlungsstärke: $\downarrow \downarrow]$

\section{Empfehlung 2.22}

Eine endoskopische Evaluation mittels Endoskopie kann durchgeführt werden bei schwerem akutem Schub und bei therapierefraktären Verläufen zur Bestätigung der aktiven Erkrankung und zum Ausschluss von infektiösen Komplikationen.

[Evidenzgrad: D, Konsensusstärke: starker Konsens, Empfehlungsstärke: $\uparrow$ ]

\section{Erläuterung}

Unabhängig von der Frage der Krankheitsausdehnung bei der Festlegung der Prognose, dem Krebsrisiko und der Wahl der Therapie wurde die Notwendigkeit regelmäßiger Wiederholungsuntersuchungen nach einer Indexkoloskopie nie untersucht. Die Koloskopie ist deutlich sensitiver als Kontrastmitteluntersuchungen, um die Krankheitsausdehnung abzuschätzen. Die Abschätzung des Malignitätsrisikos basiert aber historisch gesehen auf Kontrastmitteluntersuchungen, und die Koloskopie definiert eine andere Ausdehnung im Vergleich zur Histopathologie [92, 97-99]. Die Chromoendoskopie korreliert besser mit der histopathologischen Krankheitsausdehnung, aber das Verfahren ist zeitaufwendig und erfordert eine Expertise, die nicht universell verfügbar ist [100]. Eine medikamentös induzierte klinische Remission muss nicht zwingend mit einer endoskopisch oder histologisch erfassten Remission einhergehen; die prognostischen Konsequenzen einer endoskopischen Reevaluation in klinischer Remission sind noch nicht abschließend zu beurteilen [92]. Es mehren sich jedoch die Hinweise darauf, dass die sogenannte Mukosaheilung bei der Colitis ulcerosa eine prognostische Bedeutung hat und eine endoskopische Kontrolle nach einer medikamentösen Remissionsinduktion daher relevante Informationen liefern kann [101-103].

\section{Differenzialdiagnostische Abgrenzung zum Morbus Crohn}

\section{Empfehlung 2.23}

Bei nicht eindeutig zu klassifizierender Colitis und zum Ausschluss eines Morbus Crohn sollte eine Diagnostik des oberen und mittleren Gastrointestinaltrakts mittels Ösophagogastroduodenoskopie (mit Biopsien) und mittels MRT des Dünndarms durchgeführt werden.

[Evidenzgrad: D, Konsensusstärke: starker Konsens, Empfehlungsstärke: $\uparrow$ ]

\section{Erläuterung}

Bei diagnostischen Unklarheiten (z.B. Aussparung des Rektums, ungewöhnliche Symptome, endoskopische Hinweise für eine Backwash-Ileitis) sollte differenzialdiagnostisch das Vorliegen eines Morbus Crohn bedacht werden und in Abhängigkeit vom klinischen Kontext eine entsprechende Diagnostik des oberen und mittleren Verdauungstrakts durchgeführt werden. Hierbei sollte analog zu den bestehenden DGVS-Leitlinien zur Diagnostik und Therapie des Morbus Crohn verfahren werden [104].

\section{Bestimmung der Krankheitsaktivität}

\section{Empfehlung 2.24}

Die Anwendung klinischer und/oder endoskopischer Aktivitätsindizes kann hilfreich sein, um ein Therapieansprechen zu quantifizieren und Patientenverläufe zu objektivieren.

[Evidenzgrad: D, Konsensusstärke: starker Konsens, Empfehlungsstärke: $\uparrow$ ]

\section{Erläuterung}

Klinische, endoskopische oder kombinierte Aktivitätsindizes für die Colitis ulcerosa sind verfügbar, keiner von ihnen wurde jedoch bisher adäquat validiert [105]. In der täglichen Routine 
werden diese Indizes selten eingesetzt. Gegenwärtig werden Krankheitsaktivitätsindizes bei Colitis ulcerosa überwiegend nur im Rahmen von klinischen Studien eingesetzt.

Die Klassifikation einer schweren Colitis ulcerosa nach Truelove und Witts aus dem Jahr 1955 [106] wurde bis heute weitgehend beibehalten, da sie einfach zu merken und anzuwenden ist. Diese Klassifikation wird immer noch als das Verfahren der Wahl angesehen, um Patienten zu identifizieren, die eine sofortige Krankenhauseinweisung und eine intensive Therapie benötigen [107]. Für pädiatrische Patienten hat sich der PUCAI nach Turner etabliert [108].

\section{Ultraschall}

\section{Empfehlung 2.25*}

Die hochauflösende abdominelle Sonografie soll Bestandteil der Diagnostik bei der Erstdiagnose sowie einem schweren akuten Schub zur Erfassung des Befallsmusters und insbesondere bei Verdacht auf Komplikationen sein.

[Evidenzgrad: B, Konsensusstärke: Konsens, Empfehlungsstärke: $\uparrow \uparrow]$

\section{Erläuterung}

Der transabdominelle Ultraschall kann mit einer Sensitivität von bis zu 90\% Entzündungen im Bereich des Dickdarms detektieren. Die Sonografie hat den Vorteil, preiswert und nicht invasiv zu sein, allerdings ist die Genauigkeit des Verfahrens stark abhängig von der Erfahrung des Untersuchers. Darüber hinaus besteht eine geringe Spezifität zur Differenzierung einer Colitis ulcerosa von anderen Ursachen einer Dickdarmentzündung [109-111]. In der Hand des geübten Untersuchers kann sowohl die Aktivität der Erkrankung als auch die Krankheitsausdehnung zuverlässig bestimmt werden [112-118]. Neuere Untersuchungen zeigen, dass eine gute Korrelation der Sonografie mit der endoskopischen Aktivität besteht und durch die gute Korrelation mit dem Therapieansprechen die Methode auch als prognostisches Verfahren angewandt werden kann [119]. Hydrokolonischer Ultraschall (abdomineller Ultraschall im Zusammenhang mit retrograder Insufflation von Wasser in das Kolon) hat eine höhere Sensitivität zur Identifizierung einer aktiven Colitis, allerdings ist diese Methode zu aufwendig für die tägliche klinische Praxis [120]. Die Doppler-Sonografie der Arterien mesenterica superior und inferior wurde angewandt, um die Krankheitsaktivität und das Risiko eines Rezidivs zu evaluieren. Sie kann jedoch zum gegenwärtigen Zeitpunkt nicht als Standardverfahren verstanden werden $[121,122]$.

\section{Kolonstenose bei Colitis ulcerosa}

\section{Empfehlung 2.26*}

Da das Vorliegen einer Kolonstenose bei Colitis ulcerosa malignitätsverdächtig ist, soll eine ausgiebige Biopsieentnahme aus dem Bereich der Stenose erfolgen.

[Evidenzgrad: D, Konsensusstärke: Konsens, Empfehlungsstärke: $\uparrow \uparrow$ ]

\section{Empfehlung 2.27*}

Bei unklarer Dignität einer Kolonstenose soll die Entscheidung zur Operation großzügig gestellt werden.

[Evidenzgrad: D, Konsensusstärke: Konsens, Empfehlungsstärke: $\uparrow \uparrow]$

\section{Empfehlung 2.28}

Falls eine endoskopische Passage der Stenose unklarer Dignität nicht möglich ist, sollte eine weiterführende radiologische Diagnostik mittels Computertomografie oder Magnetresonanztomografie erfolgen.

[Evidenzgrad: D, Konsensusstärke: Konsens, Empfehlungsstärke: $\uparrow$ ]

\section{Erläuterung}

Bei langjähriger Colitis ulcerosa ist eine Kolonstriktur bzw. -stenose als ein Hinweis auf das Vorliegen eines kolorektalen Karzinoms zu interpretieren; dieser erfordert daher eine konsequente histologische Abklärung [123]. Aus dem Grund wird eine starke Empfehlung als gerechtfertigt angesehen. Wenn eine Koloskopie aufgrund einer Stenose bzw. Striktur inkomplett ist, sollte eine CT- oder MR-Kolonografie durchgeführt werden. Eine CT-Kolonografie oder eine MR-Kolonografie kann die Schleimhautbeschaffenheit und Ausdehnung der Kolitis proximal der Striktur identifizieren, möglicherweise jedoch nicht alle Läsionen, die im Rahmen einer Koloskopie sichtbar sind, zur Darstellung bringen [124, 125]. Bei unklaren Befunden sollte die chirurgische Resektion erfolgen. Trotz der schlechten Evidenzlage wurde hier ein starke Empfehlung gegeben, um jede Verzögerung einer Operation angesichts eines möglichen Kolonkarzinoms zu vermeiden.

\section{Pädiatrie}

\section{Empfehlung 2.29}

Die Diagnose einer Colitis ulcerosa soll bei Kindern bei Vorliegen von chronischen (> 4 Wochen) oder rezidivierenden (> 2 Episoden innerhalb von 6 Monaten) blutigen Durchfällen nach Ausschluss einer infektiösen Genese in Betracht gezogen werden.

[Evidenzgrad: D, Konsensusstärke: Konsens, Empfehlungsstärke: $\uparrow$ ]

\section{Erläuterung}

Kinder mit einer Colitis ulcerosa weisen in der Regel typische Symptome wie Anämie (84\%), chronische Diarrhö (74\%) und abdominelle Schmerzen, vorwiegend in Form von Tenesmen (62\%), auf [126]. Bei chronischen ( $>2$ Wochen) oder rezidvierenden Durchfällen sollte auch ohne Blutbeimengungen eine Abklärung erfolgen. Gewichtsverlust ist weniger typisch bei der Colitis ulcerosa (35\%) als beim Morbus Crohn (58\%). Das häufigste extraintestinale Symptom stellen Arthropathien dar (10\%). Hautmanifestationen sind selten. Gegenüber erwachsenen Patienten liegt in drei Viertel der Fälle eine ausgedehnte Colitis ulcerosa vor, während ein distaler Befall die Ausnahme darstellt. Über 50\% der Patienten mit Colitis ulcerosa weisen jährlich mindestens ein Rezidiv auf. Die Diagnose einer chronisch entzündlichen Darmerkrankung wird bestätigt durch klinische Evaluation und eine Kombination von biochemischen, 
endoskopischen, histologischen und bei Verdacht auf Morbus Crohn auch radiologischen (MRT-Enterografie oder -Enteroklysma) Untersuchungen. Die diagnostischen Kriterien entsprechen denen bei erwachsenen Patienten [55].

\section{Empfehlung 2.30}

Die initiale Diagnostik bei Kindern und Jugendlichen mit Verdacht auf eine chronisch entzündliche Darmerkrankung soll eine lleokoloskopie mit Entnahme von Stufenbiopsien beinhalten. Im gleichen Untersuchungsgang soll eine Ösophagogastroduodenoskopie mit Entnahme von Stufenbiopsien erfolgen.

[Evidenzgrad: D, Konsensusstärke: Konsens, Empfehlungsstärke: $\uparrow \uparrow]$

\section{Erläuterung}

Für das diagnostische Vorgehen wurden von einer Arbeitsgruppe der European Society of Pediatric Gastroenterology, Hepatology and Nutrition (ESPGHAN) Empfehlungen festgelegt [45]. Kinder mit Verdacht auf eine chronisch entzündliche Darmerkrankung sollen eine komplette Koloskopie mit Intubation des terminalen Ileums und Entnahme von Stufenbiopsien (terminales Ileum, Coecum, Colon ascendens, Colon transversum, Colon descendens, Sigma und Rektum) sowie eine Ösophagogastroduodenoskopie mit Stufenbiopsien aus dem Ösophagus, Magen und Duodenum erhalten [45]. Nur bei eindeutiger Zuordnung zur Colitis ulcerosa kann auf eine Bildgebung des Dünndarms (in der Regel eine MRT-Enterografie) verzichtet werden. Im Unterschied $\mathrm{zu}$ erwachsenen Patienten haben drei Viertel der pädiatrischen Patienten eine ausgedehnte Kolitis, sodass eine komplette Koloskopie obligat ist. Eine alleinige Sigmoidoskopie ist in der Regel nicht indiziert, mit Ausnahme einer schweren Colitis ulcerosa, bei der das Risiko einer Darmperforation erhöht ist. Die Endoskopie sollte bei pädiatrischen Patienten in Vollnarkose oder tiefer Analgosedierung durchgeführt werden.

Bei Beginn der Colitis in den ersten 3 Lebensjahren, bei perianalen Läsionen, einer auffälligen Anamnese für häufige oder ungewöhnliche Infektionen unabhängig vom Alter, und Konsanguinität der Eltern sind angeborene Immundefektzustände als Ursache der Colitis durch entsprechende immunologische Untersuchungen auszuschließen. Eine allergische Colitis oder eine Nahrungsmittelallergie als modulierender Faktor der entzündlichen Aktivität sind besonders bei jüngenen Kindern mit Colitis differenzialdiagnostisch zu bedenken.

\section{Kapitel 3: Histopathologische Diagnostik \\ $\nabla$}

\section{Entzündungsdiagnostik}

\section{Empfehlung 3.1}

Die histologische Untersuchung von endoskopischen Mukosabiopsien stellt einen wichtigen Baustein zur Diagnose einer Colitis ulcerosa dar. Zur Erstdiagnose einer Colitis ulcerosa sollen multiple Biopsienentnahmen aus dem terminalen lleum und jedem Kolonsegment unter Einschluss des Rektums (Einsendung in getrennten Probengefäßen) untersucht werden.

[Evidenzgrad: B, Konsensusstärke: starker Konsens, Empfehlungsstärke: $\uparrow \uparrow]$

\section{Empfehlung 3.2}

Histopathologische Kriterien, die bei der Beurteilung von Biopsien zur Diagnose einer Colitis ulcerosa herangezogen werden sollen, sind:

- diffuse panmukosale chronische Entzündung (Lymphozyten und Plasmazellen) in Kombination mit einer Störung der Kryptenarchitektur/Kryptenatrophie,

- Plasmozytose im basalen Schleimhautstroma,

- Panethzell-Metaplasien distal der rechten Kolonflexur,

- Reduktion der Anzahl von Becherzellen bzw. des Muzingehalts der Einzelzelle,

- kontinuierliche Verteilung der entzündlichen und strukturellen Schleimhautveränderungen, abnehmender Gradient von distal nach proximal.

[Evidenzgrad: D, Konsensusstärke: starker Konsens, Empfehlungsstärke: $\uparrow \uparrow$ ]

\section{Empfehlung 3.3}

Im Initialstadium einer Colitis ulcerosa (Dauer < 4-6 Wochen) kann eine Störung der Krytenarchitektur/Kryptenatrophie fehlen. Der Nachweis einer basalen Plasmozytose kann in diesem Kontext als Frühzeichen einer potenziellen chronisch-entzündlichen Darmerkrankung gewertet werden.

[Evidenzgrad: B, Konsensusstärke: Konsens, Empfehlungsstärke: $\uparrow]$

\section{Empfehlung 3.4}

Abweichende morphologische Befundmuster können bei der Colitis ulcerosa vorkommen und sollen speziell bei pädiatrischen Patienten (jünger als 10 - 12 jahre) berücksichtigt werden.

[Evidenzgrad: D, Konsensusstärke: starker Konsens, Empfehlungsstärke: $\uparrow \uparrow$ ]

\section{Empfehlung 3.5}

Die bioptischen Proben sollen bez. der Lokalisation gekennzeichnet sein und durch Informationen zum klinischen Bild ergänzt werden (Endoskopiebefund, Art und Dauer der Symptomatik, Art und Dauer der Behandlung).

[Evidenzgrad: D, Konsensusstärke: starker Konsens, Empfehlungsstärke: $\uparrow \uparrow$ ]

\section{Empfehlung 3.6}

Die histologische Aufarbeitung der Proben soll in Stufen- oder Serienschnitten erfolgen.

[Evidenzgrad: D, Konsensusstärke: starker Konsens, Empfehlungsstärke: $\uparrow \uparrow$ ]

\section{Empfehlung 3.7}

Der Pathologiebefund soll eine Aussage zur histologischen Entzündungsaktivität enthalten.

[Evidenzgrad: D, Konsensusstärke: starker Konsens, Empfehlungsstärke: $\uparrow \uparrow$ ] 


\section{Erläuterung}

Die Diagnose einer Colitis ulcerosa basiert auf dem Nachweis einer charakteristischen Kombination von klinischen, laborchemischen, endoskopischen, radiologischen und pathomorphologischen Befunden. Einen Goldstandard für die Diagnose gibt es nicht. Auch die pathohistologische Diagnostik beruht auf der synoptischen Beurteilung einer Kombination von Charakteristika, die schwerpunktmäßig die Art und Verteilung der Entzündungsinfiltrate sowie Veränderungen der Mukosaarchitektur betreffen [127-140]. Die histologischen Einzelbefunde sind hierbei für sich genommen nicht spezifisch und können z.T. auch bei anderen Formen entzündlicher Darmerkrankungen auftreten.

Veränderungen der Mukosaarchitektur bei Colitis ulcerosa beinhalten Irregularitäten in Form, Orientierung und Größe der Krypten ( $>10 \%$ der Krypten; mehr als 2 verzweigte, nicht parallel orientierte Krypten in einer Biopsie) [134, 136, 138, 140]. Der Begriff Kryptenatrophie bezeichnet den Befund einer verminderten Kryptendichte (Distanz von mehr als einem Kryptenquerschnitt zwischen 2 benachbarten Krypten) und/ oder einer Distanzbildung zwischen Kryptenbasis und der Lamina muscularis mucosae, zumeist verbunden mit einem basal vermehrten mononukleären, plasmazellreichen Infiltrat [132, 133, 136, 138]. Der Terminus „transmukosale Entzündung“ beinhaltet einen diffus gesteigerten Gehalt an mononukleären Zellen in der Lamina propria unter Einbeziehung der mittleren und basalen Schleimhautabschnitte [132, 136]. Eine basale Plasmozytose ist definiert als Nachweis von Plasmazellen in den basalen Bezirken (1/5) der Lamina propria oder zwischen der Kryptenbasis und der Lamina muscularis mucosae (subkryptal) [133, 134].

Die bioptische Diagnose einer Colitis ulcerosa in Abgrenzung zum Morbus Crohn und anderen entzündlichen Darmerkrankungen stützt sich insbesondere auf die Beurteilung des Ausmaßes (ausgeprägt, diffus) und der topografischen Verteilung (kontinuierliche Verteilung, abnehmender Gradient von distal nach proximal) der in Punkt 3.2 der Empfehlung genannten histopathologischen Kriterien. Daher sollen im Rahmen der Erstdiagnostik Stufenbiopsien aus dem terminalen Ileum und aus allen Kolonabschnitten unter Einbeziehung des Rektums gewonnen werden und die bioptischen Proben bez. ihrer Lokalisation gekennzeichnet sein. Die diagnostische Aussagekraft kann so im Vergleich zu singulären bzw. nicht systematischen Biopsien deutlich gesteigert werden [128, 129, 141-144]. Zum Ausschluss differenzialdiagnostisch relevanter Befunde (z.B. epitheloidzellige Granulome) ist eine Aufarbeitung der Proben in Stufen- oder Serienschnitten zu empfehlen [145, 146].

Unter diesen Voraussetzungen kann die histopathologische Diagnose einer Colitis ulcerosa an Mukosabiopsien mit einer Sensitivität und Spezifität von mehr als 70\% [128-130, 135, $140]$, in einigen Studien von bis über 90\% gestellt werden [137-139], zumindest bei aktiver Erkrankung. Einige Autoren propagieren die Beurteilung anhand von standardisierten Score-Schemata mit numerischen Koeffizienten [137-139]. Die Diagnose erfordert den Nachweis von zwei bis drei der genannten histomorphologischen Kriterien.

Im Initialstadium der Erkrankung (Krankheitsdauer $<4$ - 6 Wochen) kann eine Störung der Mukosaarchitektur fehlen, eine spezifische Diagnose ist dann gegebenenfalls nicht möglich. Der Befund einer basalen Plasmozytose ist in diesem Kontext von prädiktiver Relevanz und kann als Frühzeichen einer po- tenziellen chronisch-entzündlichen Darmerkrankung gewertet werden [133, 134, 147, 148].

In diagnostischer Hinsicht ist zu berücksichtigen, dass das morphologische Erscheinungsbild chronisch-entzündlicher Darmerkrankungen biologischen Variationen unterliegt (phasenhafter Verlauf) und zudem durch die Therapie beeinflusst wird [149, 150]. So kann es im Verlauf einer Colitis ulcerosa durchaus zur diskontinuierlichen Ausprägung des Entzündungsbilds, auch mit Aussparung des Rektums, kommen [151]. Insbesondere bei pädiatrischen CED-Patienten (jünger als 10 Jahre) ist auch ohne vorangegangene Therapie mit abweichenden Mustern zu rechnen [152-155]. Informationen zum klinischen Bild (Anamnese, Erkrankungsdauer, Art und Dauer der Therapie, Endoskopiebefund) sind daher für eine effiziente Beurteilung erforderlich und verbessern die Validität der histologischen Befundeinordnung [129]. Dies gilt insbesondere auch im Hinblick auf die differenzialdiagnostische Abgrenzung der Colitis ulcerosa von anderweitigen, ätiologisch oder phänotypisch definierten Entzündungsformen (infektiöse Colitiden, medikamentös induzierte Colitiden, Diversionscolitis, Divertikelkrankheits-assoziierte Colitis, Allergie-assoziierte Colitis u.a.) $[156-158]$.

Die histopathologische Einordnung der Entzündungsaktivität bei der Colitis ulcerosa orientiert sich am Ausmaß der Gewebeinfiltration durch segmentkernige neutrophile Granulozyten und der hiermit assoziierten Schädigung des Darmepithels mit neutrophiler Epithelinvasion, Ausbildung von Kryptitisherden und Kryptenabszessen bis hin zu erosiven und ulzerösen Läsionen [132, 135, 136, 138]. Histologischer Befund und klinische Krankheitsaktivität korrelieren beim individuellen Patienten nur bedingt miteinander [159]. Aus Therapiestudien gibt es allerdings Hinweise auf die Assoziation zwischen klinischer Besserung und Rückgang aktiver histologischer Veränderungen [160]. Ein aktives morphologisches Bild ist mit dem Auftreten rezidivierender Erkrankungsschübe assoziiert [161-163]. Eine Aussage zur histologischen Entzündungsaktivität im Pathologiebefund ist daher besonders im Hinblick auf die Verlaufsbeurteilung von Interesse. Verschiedene Graduierungsschemata sind etabliert (Riley-Score u.a.). Der potenzielle Nutzen der Histopathologie für die Vorhersage eines Rezidivs und der Beurteilung der adäquaten Kontrolle der Entzündung hat Implikationen für das therapeutische Management und die Verringerung des Neoplasie-Risikos.

\section{Intraepitheliale Neoplasien (IEN)}

\section{Empfehlung 3.8}

Die Diagnose von intraepithelialen Neoplasien/Dysplasien bei der Colitis ulcerosa soll nach den seit 2010 gültigen Kriterien der WHO erfolgen; IEN/Dysplasien sollen histopathologisch graduiert werden (niedriger oder hoher Grad).

[Evidenzgrad: D, Konsensusstärke: starker Konsens, Empfehlungsstärke: $\uparrow \uparrow]$

\section{Empfehlung 3.9}

Bei histologischer Diagnose jeder IEN/Dysplasie soll stets eine externe Zweitbeurteilung eingeholt werden.

[Evidenzgrad: C, Konsensusstärke: starker Konsens, Empfehlungsstärke: $\uparrow \uparrow$ ] 
Tab. 8 Nach $[177,178]$.

\begin{tabular}{|c|c|c|}
\hline Eigenschaft & Adenom & DALM \\
\hline Makroskopie & zirkumskripter Polyp & $\begin{array}{l}\text { Plaque-artige } \\
\text { Veränderung }\end{array}$ \\
\hline Drüsen & $\begin{array}{l}\text { gleichmäßig, rund oder } \\
\text { oval }\end{array}$ & unregelmäßig \\
\hline Drüsengröße & gleich groß & größenvariabel \\
\hline intrazelluläres Muzin & gleichmäßig verteilt & variabel \\
\hline Kerne & $\begin{array}{l}\text { gleichmäßig stäbchen- } \\
\text { förmig }\end{array}$ & rund und variabel \\
\hline Stroma & wenig & unterschiedlich \\
\hline Proliferationszone & luminal & basal \\
\hline $\begin{array}{l}\text { Übergang zur an- } \\
\text { grenzenden Mukosa }\end{array}$ & scharf & graduell, kontinuierlich \\
\hline
\end{tabular}

\section{Empfehlung 3.10*}

Im Falle einer erhabenen Läsion mit IEN/Dysplasie soll eine Unterscheidung zwischen einer CED-assoziierten sogenannten DALMLäsion (Dysplasie-assoziierte Läsion oder Masse) oder einem sporadischen Adenom beziehungsweise einer adenomartigen IEN/Dysplasie (ALM, „adenoma-like mass“) jeweils mit Angabe des IENbzw. Dysplasiegrads (LGIEN oder HGIEN) erfolgen, da diese Aussage von therapeutischer Bedeutung ist. Diese Unterscheidung soll unter Berücksichtigung des makroskopischen bzw. endoskopischen Befunds erfolgen.

[Evidenzgrad: C, Konsensusstärke: Konsens, Empfehlungsstärke: $\uparrow \uparrow]$

\section{Erläuterung}

Die Angabe des Grades der IEN/Dysplasien ist wichtig, da damit eine Aussage über die Sensitivität und Spezifität zum Risiko eines syn- oder metachronen kolorektalen Karzinoms verbunden ist. Der Nachweis einer IEN, unabhängig vom Grad, korreliert mit einer Sensitivität und Spezifität von 74\% mit einer KRK-Entstehung, während in der gleichen Studie eine hochgradige IEN/ Dysplasien zwar eine geringere Sensitivität (34\%), aber mit 98\% eine höhere Spezifität für einen KRK-Nachweis zeigte [164]. In einer aktuellsten Metaanalyse war eine niedriggradige IEN mit einem 9-fach erhöhten KRK-Risiko und mit einem 12-fach erhöhten Risiko für eine fortgeschrittene Neoplasie korreliert [165]. Die Diagnose einer niedriggradigen IEN/Dysplasien ist daher mit einem substanziellen Karzinomrisiko assoziiert und hat erhebliche prognostische Implikationen [166-168].

Da eine hohe Interobserver-Variabilität besteht [169, 170, 571], sollte jede histopathologische „IEN/Dysplasie“-Diagnose von einem erfahrenen externen Pathologen durch eine Zweitbeurteilung bestätigt werden. Überdies sollten Einzelstudien, in denen kein erhöhtes Malignom-Risiko bei niedriggradiger IEN gefunden wurde, im Kontext der Metaanalysen betrachtet werden [171].

Die Colitis-ulcerosa-assoziierte intraepitheliale Neoplasie (insbesondere die DALM = Dysplasie-assoziierte Läsion oder Masse) sollte anhand makroskopischer und mikroskopischer Kriterien von sporadischen Adenomen mit IEN abgegrenzt werden. Bei der Colitis ulcerosa ist (wie auch beim Morbus Crohn) diese Unterscheidung notwendig, da sich das klinische Management sporadischer Adenome von dem Kolitis-assoziierter intraepithelialer Neoplasien unterscheidet (lokale Entfernung im Gesunden vs. Proktokolektomie; vgl. auch Kapitel 7). Dem makro- skopischen Aspekt (zirkumskripte vs. Plaque-artige Läsion) kommt hierbei besondere Bedeutung zu, daher muss der Endoskopiebefund in die differenzialdiagnostischen Überlegungen mit einbezogen werden. Weitere morphologische Kriterien zur Unterscheidung zwischen Adenom und DALM sind in $\bullet$ Tab. 8 aufgelistet.

Das Patientenalter, die Lokalisation und Morphologie der Läsion sowie Biopsien aus der umgebenden flachen Mukosa können bei der Entscheidungsfindung hilfreich sein. Im Einzelfall kann diese Unterscheidung jedoch schwierig oder unmöglich sein [172-178].

\section{Kapitel 4: Akuter Schub}

\section{$\nabla$}

Der akute Schub einer Colitis ulcerosa ist durch die typischen Beschwerden (blutige Diarrhöen, Tenesmen, imperativer Stuhldrang) charakterisiert. Bezüglich der notwendigen diagnostischen Maßnahmen wird auf Kapitel 2 verwiesen.

Bei der Auswahl geeigneter Therapien müssen Krankheitsaktivität, Befallsmuster, extraintestinale Manifestationen, Krankheitsverlauf, Vormedikation, Begleitmedikationen, Wirksamkeit von vorangegangen Therapieversuchen, Nebenwirkungsprofile, allgemeine Begleitumstände und anderweitige Krankheitsmanifestationen Berücksichtigung finden. Die unterschiedlichen Therapieoptionen sollten mit ihren Vor- und Nachteilen individuell mit den Patienten besprochen werden.

Grundsätzlich ist die Therapie an die Möglichkeiten und Vorstellungen des einzelnen Patienten anzupassen. So sind insbesondere bei der Abwägung zwischen ambulanter und stationärer Versorgung die Möglichkeiten von Arzt und Patient besonders $\mathrm{zu}$ beachten.

Da sich die Therapie im Wesentlichen nach der Ausbreitung und der Schwere der Erkrankung richtet, ist der Abschnitt „Akuter Schub“ entsprechend gegliedert.

\section{Proktitis}

\section{Empfehlung 4.1*}

Eine leichte bis mäßig aktive Proktitis soll zunächst mit 5-Aminosalizylaten $\geq 500 \mathrm{mg} / \mathrm{d}$ (bei Kindern $\geq 250 \mathrm{mg} / \mathrm{d}$ ) als Suppositorium behandelt werden.

[Evidenzgrad: B, Konsensusstärke: starker Konsens, Empfehlungsstärke: $\uparrow \uparrow$ ]

\section{Empfehlung 4.2}

Mesalazinschaum und Mesalazineinläufe stellen eine äquivalente therapeutische Alternative dar.

[Evidenzgrad: B, Konsensusstärke: starker Konsens, Empfehlungsstärke: $\uparrow$ ]

\section{Empfehlung 4.3*}

Bei Versagen der Monotherapie soll die Kombination der rektalen Mesalazinanwendung mit topischen Steroiden oder der oralen Gabe von 5-ASA-freisetzenden Präparaten eingesetzt werden.

[Evidenzgrad: B, Konsensusstärke: Konsens, Empfehlungsstärke: $\uparrow \uparrow]$ 


\section{Erläuterung}

Beschränkt sich eine aktive Colitis ulcerosa auf das Rektum, so ist zunächst eine topische Therapie mit 5-Aminosalicylaten (5ASA) indiziert. Hierbei sollten zunächst Suppositorien genutzt werden, da sie einfach zu verwenden sind [179] und den Wirkstoff gegenüber Schäumen und Einläufen günstiger freisetzen [180-182]. Die Wirksamkeit der rektalen 5-ASA-Therapie konnte in Studien mehrfach belegt werden [183]. Eine ausführliche Metaanalyse konnte zeigen, dass die rektale Gabe von 5-ASA und Kortikoidsteroiden entweder allein oder in Kombination mit oralem 5-ASA die wirksamste Therapieform ist [184]. Bei unzureichendem Ansprechen auf eine rektale Therapie mit 5ASA sollte eine kombinierte Therapie aus rektaler 5-ASA- und oraler 5-ASA-Therapie oder eine Kombination mit rektalen Steroiden in Betracht gezogen werden. Es muss betont werden, dass keine Studien vorliegen, die eine Kombinationstherapie der Proktitis untersucht haben. In der Regel wurde in diesen Studien die distale Colitis ulcerosa/Linksseitenkolitis untersucht, sodass hier die Ergebnisse übertragen wurden.

Bei der Behandlung von Kindern kann eine tägliche Dosis von $250 \mathrm{mg}$ 5-ASA ausreichend sein. Mesalazinschäume und Mesalazineinläufe stellen eine wirksame Therapiealternative zur Gabe von Suppositorien dar [184].

Zusammenfassend kann festgehalten werden, dass initial eine rektale 5-ASA-Therapie die Therapie der ersten Wahl darstellt. Als Zweitlinientherapie oder Therapiealternative kommen topische Kortikosteroide oder eine Kombination der rektalen 5-Aminosalicylat-Therapie mit einer oralen 5-Aminosalicylat-Therapie infrage. Sollten die o.g. Therapien nicht den erwünschten Erfolg bringen, werden die Therapieprinzipien der schweren Colitis ulcerosa mit beliebiger Ausdehnung angewendet (siehe unten). Somit ist als nächster Schritt die systemische Steroidgabe indiziert. Dies gilt insbesondere dann, wenn weiterhin (nach spätestens 14 Tagen) blutige Stuhlgänge auftreten.

Die Therapie der refraktären Proktitis kann eine erhebliche klinische Herausforderung darstellen, weil systemische Therapieformen oder eine Operation unter Umständen, auch unter Abwägung von Risiken und therapeutischem Nutzen, eine Übertherapie darstellen können. Bei Versagen der konventionellen Therapie kann eine topische, rektale Anwendung von Tacrolimus eine Therapiealternative darstellen [185]. Eine sehr kleine prospektive Pilotstudie konnte ein gutes Ansprechen auf diese Therapieform zeigen ( 6 von 8 Patienten erreichten eine Remission nach 8 Wochen). Eine weitere kleine Studie mit 17 CU-Patienten mit Proktitis und Linksseitenkolitis bestätigte diese Erfahrungen [186]. Eine solche Therapie sollte allerdings in einem Zentrum mit der entsprechenden Erfahrung in der Therapie der refraktären Proktitis erfolgen.

Die Pflege der Perianalregion hat einen großen und häufig unterschätzten Stellenwert in der Therapie der CU. Neben einer Optimierung der hygienischen Maßnahmen sollten auch pflegende und barrierebildende Externa ihre Anwendung finden.

\section{Linksseitenkolitis}

\section{Empfehlung $4.4^{*}$}

Eine leichte bis mäßig schwere linksseitige CU soll initial mit rektalen 5-ASA in Form von Einläufen oder Schäumen ( $\geq 1 \mathrm{~g} / \mathrm{d}$ ) in Kombination mit oralen 5-ASA-freisetzenden Präparaten ( $\geq 3 \mathrm{~g} / \mathrm{d}$ ) behandelt werden.

[Evidenzgrad: B, Konsensusstärke: Konsens, Empfehlungsstärke: $\uparrow \uparrow]$

\section{Empfehlung 4.5}

Eine alleinige orale Gabe von 5-ASA-freisetzenden Präparaten $(\geq 3 \mathrm{~g} / \mathrm{d})$ kann alternativ eingesetzt werden, ist aber weniger wirksam.

[Evidenzgrad: B, Konsensusstärke: starker Konsens, Empfehlungsstärke: $\uparrow$ ]

\section{Empfehlung $4.6^{*}$}

Die rektale Anwendung von 5-ASA-Einläufen oder -Schäumen $(\geq 1 \mathrm{~g} / \mathrm{d}$ ) soll der topischen Steroidtherapie vorgezogen werden.

[Evidenzgrad: A, Konsensusstärke: starker Konsens, Empfehlungsstärke: $\uparrow \uparrow$ ]

\section{Empfehlung 4.7}

Aufgrund der besseren Therapieadhärenz und der größeren Patientenzufriedenheit kann die einmalige orale Gabe von retardiert formulierten 5-ASA-freisetzenden Präparaten gegenüber nicht retardierten vorgezogen werden.

[Evidenzgrad: B, Konsensusstärke: starker Konsens, Empfehlungsstärke: $\uparrow$ ]

\section{Empfehlung $4.8^{*}$}

Eine systemische Steroidtherapie $(0,5-1 \mathrm{mg} / \mathrm{kg} \mathrm{KG} / \mathrm{d}$ Prednisolonäquivalent) soll begonnen werden, wenn die Symptome der CU nicht auf die unter 4.4. - 4.6. genannte Therapie ansprechen. [Evidenzgrad: C, Konsensusstärke: Konsens, Empfehlungsstärke: $\uparrow \uparrow]$

\section{Erläuterung}

Die akute Linksseitenkolitis sollte zunächst mit einer Kombination aus oralen und rektalen 5-ASA-Präparaten behandelt werden. Eine Studie, die die alleinige rektale Gabe von 5-ASA mit der alleinigen oralen Therapie und der Kombination der oralen mit der rektalen Gabe verglich, konnte zeigen, dass die Kombinationstherapie effektiver als die Monotherapie ist [187]. Aus Studien zur ausgedehnteren Colitis ulcerosa kann geschlossen werden, dass höhere mukosale 5-ASA-Konzentrationen erreicht werden und dieser Effekt scheint mit einer höheren Wirksamkeit verbunden zu sein $[188,189]$. Die alleinige orale Therapie mit 5-ASA sollte somit nur bei solchen Patienten erfolgen, die einer rektalen Therapie nicht zugänglich sind. Dass auch eine alleinige orale Gabe von 5-ASA-freisetzenden Präparaten in der Therapie der Linksseitenkolitis wirksam sein kann, wurde in verschiedenen Studien gezeigt und in einer Metaanalyse zusammengefasst [184]. Eine weitere Metaanalyse verglich die Wirksamkeit von 5-ASA-Präparaten und Placebo in der Therapie der aktiven Colitis [190]. Es konnte eine deutliche Überlegenheit der 5-ASA-Präparate gegenüber Placebo gezeigt werden. Sulfasalazin und Mesalazin unterscheiden sich nicht signifikant, allerdings scheint das günstigere Nebenwirkungsprofil für eine bevorzugte Anwendung von 5-ASA bei erwachsenen Patienten zu sprechen. Die Wirksamkeit von MMX-Mesalazin bei mäßig schwerer bis schwerer Colitis ulcerosa wurde in placebokontrollierten Studien bestätigt [191, 192]. Für Mesalazingranulat konnte dies ebenfalls gezeigt werden [193]. Es scheint eine Dosisabhängigkeit hinsichtlich des Studienendpunkts „Ansprechen“ für 5-ASA-Dosen <2 g, 2-2,9g 
und $>3 \mathrm{~g}$ pro Tag zu bestehen. Für den Studienendpunkt „Remission“ konnte keine signifikante Dosisabhängigkeit gezeigt werden [190]. Die optimale tägliche Dosis ist vermutlich auch von der Wahl des Präparats abhängig. Insgesamt ist somit auf eine ausreichend hohe Dosierung bei der oralen Therapie mit 5-ASA-Präparaten zu achten. Einen signifikanten Wirksamkeitsunterschied der einzelnen 5-ASA-Präparate scheint es nicht zu geben. 5-ASA-Schaum und 5-ASA-Einläufe unterscheiden sich in ihrer Wirksamkeit bei aktiver Linksseitenkolitis nicht [194]. Nach vierwöchiger Behandlung mit $1 \mathrm{~g}$ 5-ASA als Schaum oder Einlauf lag die klinische Remissionsrate bei 68\% (Schaum) gegenüber 73\% (Einlauf). Verschiedene Studien verglichen die topische Anwendung von 5-ASA mit der topischen Anwendung von Kortikosteroiden in der aktiven Linksseitenkolitis. Einige Studien zeigten eine höhere Wirksamkeit der 5ASA-Therapie [195-197]. Eine Studie zeigte bei Proktitis ein Vorteil der Mesalazintherapie, während bei Proktosigmoiditis Hydrokortison wirksamer als Mesalazin erschien [198]. Gionchetti konnte eine Gleichwertigkeit von Beclomethasondipropionat und 5-ASA zeigen [199], was durch eine jüngst veröffentlichte Metaanalyse und eine weitere Studie bestätigt wird [200, 201].

Studien zur Therapie der Linksseitenkolitis bei Kindern mit Aminosalizylaten liegen nicht vor.

Bei Kindern gilt ebenfalls, dass die Therapie mit rektalen und oralen 5-ASA-Präparaten eine geeignete Therapie der Linksseitenkolitis darstellt. Zur Wirksamkeit oraler Aminosalizylate bei aktiver Colitis ulcerosa bei Kindern liegt nur eine prospektive Studie mit Sulfasalazin $(60 \mathrm{mg} / \mathrm{kg} / \mathrm{d})$ gegen Olsalazin $30 \mathrm{mg} / \mathrm{kg} /$ d vor [202] und retrospektive Daten vor [203, 204]. Mesalazin wird von den meisten Kindern in der galenischen Form von Pellets gut tolleriert, wenngleich bei schnellem Transit bei Kindern eine zeitgerechte Freisetzung nicht immer gewährleistet ist [205, 206]. Obwohl Sulfasalazin ein größeres Nebenwirkungsspektrum aufweist (v.a. gastrointestinale Nebenwirkung), hat es bei jungen Kindern den Vorteil, dass Tabletten zermörsert werden dürfen, falls Pellets nicht geschluckt werden. Damit besteht wie bei Olsalazin für die Bioverfügbarkeit keine Abhängigkeit von der Transitzeit. Die empfohlene Dosierung für Kinder liegt bei 50 (bis 100) $\mathrm{mg} / \mathrm{kg} \mathrm{KG/d} \mathrm{bis} \mathrm{zu} \mathrm{einem} \mathrm{Ma-}$ ximum von $6 \mathrm{~g} / \mathrm{d}$.

Dass eine fehlende Therapieadhärenz insbesondere in der 5ASA-Therapie der Colitis ulcerosa ein Problem darstellt, konnte mehrfach gezeigt werden [207-209]. Cervený et al. fanden bei $12,7 \%$ der untersuchten Patienten unter 5-ASA-Therapie kein 5-ASA oder Abbauprodukte im Urin. Es konnte gezeigt werden, dass ein signifikanter Anteil der Colitis ulcerosa-Schübe und der damit verbundenen medizinischen Kosten auf eine fehlende Therapieadhärenz zur 5-ASA-Therapie zurückgeführt werden kann [210]. Dies gilt insbesondere dann, wenn große Mengen an Medikamenten zu unterschiedlichen Zeitpunkten eingenommen werden müssen oder die Anwendung der Medikation als unangenehm wahrgenommen wird (rektale Anwendung).

In einer Studie, die die orale MMX-Mesalazin-Therapie $(3 \times 1,2 \mathrm{~g} / \mathrm{d}$ oder Placebo $)$ mit der rektalen 5-ASA-Therapie $(4 \mathrm{~g} / \mathrm{d}$ als Einlauf) in der Linksseitenkolitis verglich, wurden Unterschiede in der Compliance bei beiden Therapieformen festgestellt. Bei der oralen Therapie zeigten 97\% Compliance, bei der rektalen nur 87,5\%, wobei der Wert bei aktiver Erkrankung sogar nur bei 65,5\% lag [211]. In dieser kleinen Studie (79 Patienten) konnte kein signifikanter Unterschied in der
Wirksamkeit (klinische Remission) festgestellt werden (nach 4 bzw. 8 Wochen $57,5 / 60 \%$ in der MMX-Gruppe, 68,4/50\% in der 5-ASA-Einlauf-Gruppe). Somit scheinen retardiert formulierte 5-ASA-freisetzende orale Präparate in der Wirksamkeit 5-ASA-Einläufen nicht unterlegen. Die einmal tägliche Einnahme von solchen Präparaten und die Bevorzugung der oralen Route vor der rektalen Gabe kann in manchen Fällen eine sinnvolle Maßnahme darstellen, um die Therapieadhärenz zu erhöhen. Eine einmalige Gabe von ausreichend hochdosierten 5-ASA-Präparaten ist einer in mehrere einzelne Dosen aufgeteilten Gabe in der Wirksamkeit nicht unterlegen [191, 193, 212].

Sollten die oben aufgeführten Therapiealternativen nicht zu einem Ansprechen auf die Therapie geführt haben, muss als nächster Schritt die Gabe systemisch wirksamer Kortikosteroide in Erwägung gezogen werden. Ein deutliches Zeichen eines nicht ausreichenden Ansprechens auf die Therapie ist das Persistieren blutiger Stühle. Aller spätestens nach 14 Tagen sollten weiterführende therapeutische Maßnahmen ergriffen werden. Einzelheiten zur Anwendung von Kortikosteroiden werden im Abschnitt „Ausgedehnter Befall“ ausgeführt.

\section{Ausgedehnter Befall}

\section{Empfehlung 4.9*}

Bei ausgedehntem Befall soll eine leicht bis mäßig schwere CU zunächst mit einem oralen 5-ASA-freisetzenden Präparat in einer Dosierung $\geq 3 \mathrm{~g} / \mathrm{d}$ in Kombination mit Mesalazineinläufen oder -schäumen behandelt werden.

[Evidenzgrad: A, Konsensusstärke: starker Konsens, Empfehlungsstärke: $\uparrow \uparrow$ ]

\section{Empfehlung 4.10}

Eine systemische Steroidtherapie $(0,5-1 \mathrm{mg} / \mathrm{kg}$ Körpergewicht/ Tag Prednisolonäquivalent) soll begonnen werden, wenn die Symptome der CU nicht auf die unter $4.4-4.6$ und 4.9 genannte Therapie ansprechen.

[Evidenzgrad: C, Konsensusstärke: starker Konsens, Empfehlungsstärke: $\uparrow \uparrow$ ]

\section{Erläuterung}

Die Therapieprinzipien bei ausgedehntem Befall entsprechen im Wesentlichen denen bei der Linksseitenkolitis. Die Wirksamkeit von oralem 5-ASA ist durch Metaanalysen abgesichert [190].

Auch hier ist eine Kombinationstherapie von oralen und rektalen 5-ASA-Präparaten der Monotherapie überlegen [12, 184, 188 , 189]. Bei ungenügendem Therapieansprechen ist ein Wechsel auf eine systemische Steroidtherapie früher in Betracht zu ziehen als bei limitierterer Krankheitsausdehnung. Ein Zeitrahmen von 14 Tagen für das Abwarten eines Ansprechens auf die 5-ASA-Therapie sollte nicht wesentlich überschritten werden. Dies gilt insbesondere dann, wenn bereits eine remissionserhaltende Therapie mit 5-ASA oder Immunmodulatoren durchgeführt wird. Die Wirksamkeit von MMXMesalazin bei mäßig schwerer bis schwerer Colitis ulcerosa wurde in placebokontrollierten Studien bestätigt [191, 192]. Eine Metaanalyse verglich die Wirksamkeit von 5-ASA-Präparaten, Placebo und Sulfasalazin in der Therapie der aktiven Co- 
litis ulcerosa [190]. Es konnte eine deutliche Überlegenheit der 5-ASA-Präparate gegenüber Placebo gezeigt werden. Sulfasalazin und 5-ASA unterscheiden sich nicht signifikant, allerdings ist das Nebenwirkungsprofil von 5-ASA deutlich günstiger. Es besteht eine Dosisabhängigkeit hinsichtlich des Studienendpunkts „Ansprechen“ für 5-ASA-Dosen $<2 \mathrm{~g}, 2-2,9 \mathrm{~g}$ und $>3 \mathrm{~g}$ pro Tag. Für den Studienendpunkt „Remission“ konnte keine signifikante Dosisabhängigkeit gezeigt werden [190]. Es sollte daher auf eine ausreichend hohe Dosierung bei der oralen Therapie mit 5-ASA-Präparaten geachtet werden.

Ob die tägliche Dosis der 5-ASA-Medikation in einer oder mehreren, über den Tag verteilten Dosen eingenommen wird scheint, wie im Abschnitt Linksseitenkolitis ausgeführt, für die Wirksamkeit keine erhebliche Rolle zu spielen [191-193]. Aufgrund der verbesserten Therapieadhärenz scheint die Einmalgabe günstiger zu sein.

Das Vorgehen bei Kindern ist bei Linksseitenkolitis und ausgedehnterem Befall prinzipiell gleich. Es gilt wiederum, dass eine Therapieeskalation zu Kortikosteroiden früher in Betracht gezogen werden muss als bei limitierterem Ausbreitungsmuster. Einzelheiten zur Therapie mit Kortikosteroiden werden im Abschnitt „schwere Colitis ulcerosa, beliebige Ausdehnung“ erläutert.

\section{Schwere CU, beliebige Ausdehnung}

\section{Empfehlung 4.11}

Definition: Zur Definition einer schweren, aktiven CU können die Kriterien von Truelove und Witts (mehr als 6 blutige Durchfälle/d, Fieber, Tachykardie, Anämie, BSG $>30 \mathrm{~mm} / \mathrm{h}$ ) angewendet werden.

[Evidenzgrad: C, Konsensusstärke: Konsens, Empfehlungsstärke: $\uparrow$ ]

\section{Empfehlung 4.12}

Die Behandlung einer schweren, aktiven CU mit Zeichen einer systemischen Beteiligung sollte unter stationären Bedingungen erfolgen.

[Evidenzgrad: D, Konsensusstärke: Konsens, Empfehlungsstärke: $\uparrow$ ]

\section{Empfehlung $4.13^{*}$}

Ein schwer verlaufender Schub einer CU mit Zeichen einer systemischen Beteiligung soll mit einer intravenösen Steroidtherapie (z. B. $1 \mathrm{mg} / \mathrm{kg}$ Körpergewicht Prednisolonäquivalent pro Tag) behandelt werden.

[Evidenzgrad: B, Konsensusstärke: sehr stark, Empfehlungsstärke: $\uparrow \uparrow]$

\section{Empfehlung 4.14}

Sollte eine Steroidtherapie aufgrund einer Kontraindikation oder Intoleranz nicht infrage kommen, so kann alternativ eine Therapie mit Ciclosporin A (B), Infliximab (B) oder Tacrolimus (C) zum Einsatz kommen.

[Evidenzgrad: siehe Empfehlungstext, Konsensusstärke: Konsens, Empfehlungsstärke: $\uparrow$ ]

\section{Empfehlung 4.15}

Diese Patienten sollen intensiv überwacht und, insbesondere jene mit toxischem Verlauf, in enger Zusammenarbeit von Gastroenterologen/Kindergastroenterologen und Chirurgen betreut werden. [Evidenzgrad: D, Konsensusstärke: starker Konsens, Empfehlungsstärke: $\uparrow \uparrow$ ]

\section{Erläuterung}

Eine mehr oder weniger einfach anwendbare und im klinischen Alltag bewährte Definition der schweren Colitis ulcerosa kann anhand der Kriterien von Truelove und Witts erfolgen [106, 213]. Diese Kriterien beinhalten:

- schwere Diarrhöen mit 6 oder mehr makroskopisch blutigen Stühlen pro Tag,

- Fieber (mit einer mittleren Abendtemperatur von über $37,5^{\circ}$ Celsius oder einer Temperatur $>37,8^{\circ}$ Celsius an wenigstens 2 von 4 Tagen,

- Tachykardie mit einem Puls $>90 / \mathrm{min}$,

- Anämie mit einem Hb-Wert $<75 \%$ der Norm und

- eine BSG > $30 \mathrm{~mm} / \mathrm{h}$.

Diese Parameter bilden im Prinzip eine systemische Krankheitsaktivität $\mathrm{ab}$, die sich in der Regel auch direkt aus dem klinischen Bild ergibt. Es handelt sich hier um schwer kranke Patienten. Die Anwendung von Score-Systemen spielt hier im klinischen Alltag eher eine bestätigende Rolle und ist nicht zwingend geboten. Allerdings ist grundsätzlich auf Zeichen einer systemischen Krankheitsmanifestation zu achten. Bei Kindern sollte der PUCAI zur Anwendung kommen [108]. In den PUCAI fließen ein: Bauchschmerzen, rektaler Blutabgang, Stuhlkonsistenz, Stuhlfrequenz, nächtlicher Stuhlgang und die Aktivitätsbeeinträchtigung (Summenbildung mit einem Wert zwischen 0-85). Ein PUCAI von >65 Punkten zeigt einen schweren Kolitisschub an.

Die schwere Colitis ulcerosa stellt nach wie vor ein bedrohliches Krankheitsbild dar, obwohl die Mortalitätsrate in spezialisierten Zentren < $1 \%$ liegt [107]. Eine Metaanalyse, die das Ansprechen der schweren Colitis ulcerosa auf Kortikosteroide untersuchte, zeigte eine mittlere Proktokolektomierate von 27\% und eine Mortalitätsrate von 1\% [214]. Vor diesem Hintergrund scheint in der Regel eine Behandlung der schweren Colitis ulcerosa unter stationären Bedingungen dringend geboten. Die hier notwendige Therapie geht über die pharmakologische Therapie weit hinaus und kann in der Regel nur unter stationären Bedingungen gewährleistet werden. Parenteraler Flüssigkeits- und Elektrolytausgleich, engmaschige Laborwertkontrollen, mikrobiologische Untersuchungen, ggf. endoskopische Diagnostik, Thromboseprophylaxe, enterale und parenterale Ernährung, Schmerztherapie, die Anwendung von Externa, physio- und ggf. psychotherapeutische Betreuung, antibiotische Therapie und Bluttransfusionen können ein notwendiger Teil der Therapie sein.

Die Anwendung von Steroiden in der Behandlung der Colitis ulcerosa wurde schon vor über 50 Jahren in ersten Studien untersucht [106, 215-218]. Insgesamt wurden seit dieser Zeit viele verschiedene Dosierungen und Therapieregime angewendet. Letztendlich liegen keine Daten vor, die den Nutzen einer täglichen Prednisolondosis über $60 \mathrm{mg} / \mathrm{d}$ bei schwerer Colitis ulcerosa unterstützen [214]. Auf der Grundlage der vorliegenden Studien wird eine Dosierung von $1 \mathrm{mg} / \mathrm{kg} / \mathrm{KG}$ initial empfohlen. Sehr hohe Dosen bringen keinen zusätzlichen Nutzen, niedrigere Dosen sind unter Umständen weniger wirksam 
[214, 219]. Eine frühe Reduktion der Steroidtherapie (Therapiedauer weniger als 3 Wochen) ist mit einer erhöhten Rückfallquote verbunden. Dosen unterhalb von $15 \mathrm{mg}$ Prednisolon pro Tag gelten als ineffektiv [215]. Es existieren in unterschiedlichen Kliniken unterschiedliche Anwendungsschemata bez. der Kortikosteroide (Auswahl der Präparate, Applikationsweise, Dauer der Therapie, Beginn und Geschwindigkeit des Ausschleichens, Aufteilung der täglichen Dosis), die in der Wirksamkeit nicht im Rahmen von randomisierten Studien miteinander verglichen worden sind. Grundsätzlich kommt sowohl eine orale als auch eine intravenöse Anwendung infrage. Die geeignete Applikationsweise muss vom Einzelfall abhängig gemacht werden. Bei fraglicher enteraler Resorption ist die intravenöse Applikation der sicherere Weg.

Bei Kinder liegt das Risiko, wegen eines schweren Kolitisschubs stationär aufgenommen zu werden, bei $30-40 \%$ bis zum 16 . Lebensjahr und damit höher als bei Erwachsenen. Bei Kindern und Jugendlichen sollte Methylprednisolon einmal täglich in einer Dosis von 1-2 mg/kg/d i.v., maximal 40-60 mg/d gegeben werden [220]. Etwa 30-40\% der pädiatrischen Patienten mit scherer Colitis ulcerosa sprechen nicht auf die initiale Therapie an und benötigen eine Therapieeskalation mit Infliximab oder Cyclosporin. Bei einem PUCAI über 45 Punkten am Tag 3 der i.v. Steroidtherapie sollte eine weitere Diagnostik (Sigmoidoskopie mit Ausschluss einer CMV-Colitis) veranlasst und Vorbereitungen für eine erweiterte Therapie getroffen werden. Beträgt der PUCAI > 65 Punkte am Tag 5, empfiehlt sich, die Therapie zu erweitern, da ein Ansprechen wenig wahrscheinlich ist. Alternativ ist stehts eine Kolektomie mit Eltern und Patient zu diskutieren. Für Kinder und Jugendliche wurden von der ECCO (European Crohn's and Colitis Organization) und der ESPGHAN (European Society of Pediatric Gastroenterology, Hepatology, and Nutrition) eine separate Leitlinie zur Behandlung des schweren Schubes herausgegeben [221].

Als Alternativen bei Kontraindikationen zur Steroidtherapie oder Intoleranz kommen Infliximab, Ciclosporin A oder Tacrolimus infrage. Die Anwendung von Infliximab in der Behandlung der schweren Colitis ulcerosa wurde in mehreren Studien untersucht [223-227]. Bei der Mehrheit der in diesen Studien behandelten Patienten handelte es sich um steroidrefraktäre Krankheitsverläufe, allerdings war ein großer Teil der Patienten nicht hospitalisiert. Järnerot et al. [223] untersuchten in einer doppelblinden und randomisierten Studie 45 Patienten mit akuter, schwerer Colitis ulcerosa, die zuvor nicht auf die konventionelle Steroidtherapie angesprochen hatten. Sieben Patienten der Infliximabgruppe und 14 Patienten der Placebogruppe mussten sich innerhalb von 3 Monaten nach Randomisierung einer Proktokolektomie unterziehen (statistisch signifikanter Unterschied). Ochsenkühn et al. untersuchten die Anwendung von Infliximab bei Patienten mit akuter schwerer Colitis ulcerosa, die zuvor nicht mit Steroiden in adäquater Dosierung behandelt worden waren [225]. In dieser sehr kleinen Studie (13 Patienten) konnte ebenfalls die Wirksamkeit von Infliximab in dieser Patientengruppe nachgewiesen werden. Die Gabe von Infliximab kann dann in einer Dosierung von $5 \mathrm{mg} / \mathrm{kg} \mathrm{KG} \mathrm{zu}$ den Zeitpunkten Woche 0, 2 und 6 erfolgen. Eine Fortführung in 8 wöchentlichen Abständen als Erhaltungstherapie sollte sich, bei entsprechender Wirksamkeit, anschließen [227].

Zur Anwendung von Ciclosporin A als Therapieoption in der Behandlung der akuten schweren Colitis ulcerosa liegt eine Metaanalyse von Shibolet et al. vor, die allerdings nur zwei randomisierte, kontrollierte Studien zur Grundlage hat [228]. Diese Metaanalyse kam zu dem Schluss, dass es nur begrenzt Hinweise für einen überlegenen Nutzen von Ciclosporin A gegenüber der Standardtherapie gibt. In einer randomisierten und placebokontrollierten Studien war zuvor eine schnelle Wirksamkeit von Ciclosporin A in der Behandlung der steroidrefraktären Colitis ulcerosa beschrieben worden [229]. Mit der Frage, ob Ciclosporin A eine Alternative zur Kortikosteroidtherapie darstellen kann, befasste sich eine randomisierte, doppelt verblindete Studie von d'Haens et al. Es wurden 30 Patienten mit schwerer CU entweder mit $40 \mathrm{mg}$ Methylprednisolon/d oder mit $4 \mathrm{mg} / \mathrm{kg} \mathrm{KG} / \mathrm{d}$ Ciclosporin A intravenös behandelt. Nach 8 Tagen konnte bei 8/15 Patienten der Methylprednisolongruppe und bei 9/14 Patienten der Ciclosporin-A-Gruppe ein Therapieansprechen verzeichnet werden. Somit wurde gezeigt, dass Ciclosporin A eine wirksame Alternative zur konventionellen Therapie mit Kortikosteroiden darstellt [230].

Für die Anwendung von Tacrolimus als Therapiealternative zu Kortikosteroiden in der Behandlung der akuten schweren Colitis ulcerosa liegen nur indirekte Daten vor. Verschiedene Studien untersuchten die Wirksamkeit von Tacrolimus in der Behandlung der steroidrefraktären Colitis ulcerosa [231-234]. Aus diesen Daten und der klinischen Praxis kann geschlossen werden, dass auch Tacrolimus als Therapiealternative zur konventionellen Steroidtherapie infrage kommt. Eine spezifische Studie zu dieser Fragestellung existiert jedoch nicht.

Von herausragender Bedeutung bei der Behandlung dieser schwer kranken Patienten ist es, nicht den Zeitpunkt zu versäumen, an dem die konventionelle/medikamentöse Therapie versagt und eine chirurgische Therapie (Proktokolektomie) notwendig wird. Mit der zunehmenden Anzahl an Therapiealternativen nimmt die Gefahr der Verzögerung der notwendigen chirurgischen Therapie tendenziell weiter zu. Aus diesem Dilemma ergibt sich, dass Gastroenterologen/Kindergastroenterologen und Chirurgen sehr eng miteinander kooperieren und ständig gemeinsam den Zustand der Patienten und das Ansprechen auf die Therapie beurteilen müssen. Eine intensive Überwachung der Patienten ist hierbei unerlässlich.

\section{Colitis ulcerosa refraktär auf systemische Steroid- therapie}

\section{Empfehlung 4.16}

Zur Beurteilung des Ansprechens der systemischen Steroidtherapie sollen das klinische Bild und objektivierbare Parameter (z.B. Stuhlfrequenz, Blutbeimengungen im Stuhl, Hb-Wert, Ultraschallbefund, Endoskopiebefund) herangezogen werden.

[Evidenzgrad: D, Konsensusstärke: starker Konsens, Empfehlungsstärke: $\uparrow \uparrow$ ]

\section{Empfehlung 4.17*}

Bei nicht ausreichendem Ansprechen auf eine systemische Steroidtherapie soll Ciclosporin A (A), Infliximab (A) oder Tacrolimus (B) eingesetzt werden. Bei der Therapieentscheidung soll immer auch eine chirurgische Therapiealternative in Betracht gezogen werden (B).

[Evidenzgrad: siehe Empfehlungstext, Konsensusstärke: Konsens, Empfehlungsstärke: $\uparrow \uparrow$ ] 


\section{Empfehlung $4.18^{*}$}

Nach Ansprechen auf eine Therapie kann eine Azathioprin- oder eine 6-Mercaptopurin-Therapie eingeleitet werden.

[Evidenzgrad: B, Konsensusstärke: Konsens, Empfehlungsstärke: $\uparrow$ ]

\section{Empfehlung 4.19}

Tritt unter oben genannter Therapie eine klinische Zustandsverschlechterung ein, soll eine chirurgische Therapie durchgeführt werden. Die chirurgische Therapie kann ebenso indiziert sein, wenn nach 4-7 Tagen keine Verbesserung des klinischen Zustands eintritt.

[Evidenzgrad: D, Konsensusstärke: starker Konsens, Empfehlungsstärke: $\uparrow \uparrow]$

\section{Erläuterung}

Es wurden verschiedene Versuche unternommen, Prognosescores für den Verlauf der schweren akuten Colitis ulcerosa zu ermitteln. Diese Scores beruhen auf möglichst objektiv zu erhebenden Parametern wie Stuhlfrequenz und Entzündungsparametern. In verschiedenen Studien zeigte sich, dass bei schwerer Colitis ulcerosa die Notwendigkeit einer Proktokolektomie bei einem fehlenden Therapieansprechen nach spätestens 4-7 Tage sehr wahrscheinlich ist [214, 225, 235-237]. Die Wirksamkeit einer systemischen Steroidtherapie muss engmaschig überprüft werden, da aus einem Nichtansprechen frühzeitig Konsequenzen gezogen werden müssen. Bei einem Nichtansprechen auf die systemische Gabe von Steroiden muss entschieden werden, ob eine weitere, pharmakologische Therapie (Ciclosporin A, Tacrolimus, Infliximab) oder eine Proktokolektomie als Therapieoption infrage kommt. Eine prospektive Studie untersuchte die Therapieergebnisse in 51 aufeinanderfolgenden Patienten mit schwerer Colitis ulcerosa und konnte zeigen, dass 85\% der Patienten mit mehr als 8 Stuhlgängen pro Tag oder einer Stuhlfrequenz zwischen 3 und 8 pro Tag in Kombination mit einem CRP-Wert über $45 \mathrm{mg} / \mathrm{l}$ sich einer Kolektomie unterziehen mussten [238]. Ein CRP-Wert $\geq 25 \mathrm{mg} / \mathrm{l}$ und mehr als 4 Stuhlgänge pro Tag am 3. Tag der intensiven Therapie machen die Notwendigkeit einer Kolektomie innerhalb von 30 Tagen wahrscheinlich [237]. Bei Kindern ist der PUCAI ein geeigneter Verlaufsparameter [108]. Aber auch andere Laborparameter und radiologische Kriterien können geeignet sein [225, 236]. Einen Stellenwert in der Verlaufsbeurteilung der Kolitistherapie haben auch die Darmsonografie, die kontrastmittelverstärkte Darmsonografie und die Kernspintomografie [102, 118]. Generell gilt, dass, wenn bei einer schweren Colitis ulcerosa nach 4-7 Tagen unter konventioneller Therapie keine Verbesserung des klinischen Zustands eintritt, Therapiealternativen in Betracht gezogen werden müssen. In einer randomisierten placebokontrollierten, kleinen Studie mit Infliximab als „Rescue-Therapie“ in der schweren Colitis ulcerosa [223] wurde gezeigt, dass die Infliximabgruppe bez. des Studienendpunkts „Proktokolektomie“ signifikant günstiger lag als die Placebogruppe, bei der die hochdosierte Steroidtherapie fortgesetzt wurde. Infliximab in einer Dosis von 4-5 mg/kg/Körpergewicht stellt somit eine wirksame und sichere Behandlungsalternative in der akuten schweren Colitis ulcerosa dar, die zuvor nicht auf eine konventionelle Therapie angesprochen hat. Weitere kleinere Studien bestätigen den Nutzen von Infliximab in dieser Situation [239-242]. Letztendlich konnten 2 randomisierte, doppelt verblindete, placebokontrollierte Studien (ACT 1 und 2) generell die Wirksamkeit von Infliximab in der Behandlung der Colitis ulcerosa nachweisen [227]. In der ACT-1-Studie wurden 364 Patienten mit mäßig aktiver CU, die zuvor nicht auf Kortikosteroide und/oder Immunmodulatoren angesprochen hatte, behandelt. 69\% der Patienten, die eine Dosis von $5 \mathrm{mg} / \mathrm{kg} \mathrm{KG}$ erhielten, zeigten in Woche 8 ein klinisches Ansprechen, in der Gruppe der Patienten, die $10 \mathrm{mg} / \mathrm{kg}$ KG erhielten, waren es $61 \%$ und in der Placebogruppe 37\%, die Remissionsraten lagen bei 38,8\% (5 mg/kg KG Infliximab), 32\% $10 \mathrm{mg} \mathrm{kg}$ KG Infliximab) und 14,9\% (Placebo). Eine systematische Metaanalyse kam zu dem Ergebnis, dass Infliximab in der Behandlung der mäßig schweren bis schweren Colitis ulcerosa mit refraktärem Verlauf nach Einsatz von Kortikosteroiden und/oder Immunmodulatoren wirksam ist [224]. Eine Gabe von Infliximab zu den Zeitpunkten 0, 2 und 6 Wochen war effektiver als Placebo in der Einleitung einer klinischen Remission (Relatives Risiko [RR] 3,22, 95\%, CI2,18-4,76), in der Einleitung einer endoskopischen Remission ( $R R$ 1,88, 95\%, CI1,54-2,28) und in der Einleitung eines klinischen Therapieansprechens in Woche 8 (RR 1,99, 95\%, CI1,65-2 [214]).

Der Nutzen von Ciclosporin A in einer Dosierung von $4 \mathrm{mg} / \mathrm{kg}$ $\mathrm{KG} / \mathrm{d}$ intravenös in der Behandlung der schweren steroidrefraktären Colitis ulcerosa wurde zuerst von Lichtiger et al. in einer placebokontrollierten Studie untersucht und nachgewiesen [229]. In dieser randomisierten und placebokontrollierten, aber sehr kleinen Studie konnte bei 9 von 11 Patienten ein klinisches Therapieansprechen nach einer mittleren Dauer von 7 Tagen festgestellt werden. Spätere Studien bzw. Verlaufsbeobachtungen konnten diese ersten Ergebnisse bestätigen [240, 243, 244]. Es zeigte sich allerdings auch, dass ein großer Teil der Patienten trotz zunächst erfolgreicher Ciclosporin-Therapie früher oder später eine Proktokolektomie benötigt. Später wurden die intravenösen Dosierungen von $2 \mathrm{mg} / \mathrm{kg}$ und $4 \mathrm{mg} / \mathrm{kg}$ KG pro Tag untersucht, wobei die Ansprechraten an Tag 8 ähnlich hoch waren (86\% und 84\%) [245]. Diese Studie ist die größte randomisierte Studie zu dieser Fragestellung. Die intravenöse Dosis von $2 \mathrm{mg} / \mathrm{kg}$ KG pro Tag scheint in Abwägung von Wirksamkeit und Nebenwirkungen bei der Therapieeinleitung sinnvoll. Die optimalen therapeutischen Serumspiegel sind nicht bekannt, anzustreben sind Spiegel zwischen 250 und $400 \mathrm{ng} / \mathrm{ml}$.

Mit Tacrolimus hat ein weiterer Calcineurininhibitor Eingang in die Therapie der Colitis ulcerosa gefunden. Es liegt eine randomisierte Studie vor, die eine dosisabhängige Wirksamkeit in der Therapie in der refraktären Colitis ulcerosa zeigte [234]. Weitere kleinere Studien und Fallberichte unterstützen dieses Ergebnis [233, 246, 247]. Die Dosierung von Tacrolimus bei intravenöser Gabe liegt zwischen 0,01 und $0,02 \mathrm{mg} / \mathrm{kg}$ KG pro Tag und bei oraler Gabe zwischen 0,1 und $0,2 \mathrm{mg} / \mathrm{kg}$ KG pro Tag, verteilt auf zwei Dosen. In der Studie von Ogata et al. wurde ein Zielbereich des Talspiegels zwischen $10-15 \mathrm{ng} / \mathrm{ml}$ als optimal angegeben [234]. In einer anderen Studie schienen auch niedrigere Talspiegel $(4-8 \mathrm{ng} / \mathrm{ml})$ gut wirksam $\mathrm{zu}$ sein und weniger Nebenwirkungen zu induzieren [246].

Obwohl aufgrund der wissenschaftlichen Datenlage eine höhere Evidenz für den Einsatz von Infliximab bei der therapierefraktären Colitis ulcerosa besteht, wird allgemein empfohlen, dass bei schweren Verlaufsformen einer Colitis ulcerosa zunächst eine Therapie mit einem Calcineurininhibitor eingeleitet werden sollte. Dieses Vorgehen basiert auf der Tatsache, 
dass bei Therapieversagen des Calcineurininhibitors (Ciclosporin, Tacrolimus) diese Therapie beendet und bereits nach 1-2 Tagen ein Therapieversuch mit Infliximab begonnen werden könnte, da die immunsupprimierende Effekte der Calcineurininhibitoren aufgrund der begrenzten Halbwertzeit vernachlässigbar sind. Bei der Anwendung von Infliximab wäre aufgrund der längeren Halbwertzeit mit einer prolongierten Immunsuppression zu rechnen, sodass bei einem Therapiebeginn mit einem Calcineurininhibitor infolge von Therapieversagen von Infliximab mit einer erhöhten Immunsuppression infolge einer 3-fach-immunsuppressiven Therapie zu rechnen wäre. Bei Patienten mit einem mäßig aktiven chronisch-aktiven Verlauf einer Colitis ulcerosa, wo kein erhöhtes Risiko einer operativen Intervention besteht, kann aber durchaus auch eine primäre Anwendung von Infliximab bei einem steroidrefraktärem Verlauf einer Colitis ulcerosa erfolgen.

Eine Kombination von Infliximab, Ciclosporin A und/oder Tacrolimus wird aufgrund der zu erwartenden Nebenwirkungen nicht empfohlen. Hinsichtlich der Indikationsstellung und dem Vorgehen bei der Proktokolektomie wird auf das Kapitel 7 „Chirurgie“" verwiesen.

Eine Studie von Ardizzone et al. [248] verglich die Wirksamkeit von Azathioprin ( $2 \mathrm{mg} / \mathrm{kg} \mathrm{KG/Tag})$ und 5-ASA in der Remissionsinduktion bei steroidabhängiger Colitis ulcerosa. Es zeigte sich, dass signifikant mehr Patienten unter Azathioprin (19/36 Patienten [53\%]) als unter 5-ASA (7/36 [21\%]) eine klinische und endoskopische Remission erreichen konnten und die Kortikosteroidmedikation beenden konnten. Ein Einspareffekt für Steroide konnte gezeigt werden. Daneben existieren weitere kleinere Studien, die den therapeutischen Nutzen von Azathioprinen bei Colitis ulcerosa untersuchten [249, 250]. Letztlich sollte bei erneutem, schwerem Krankheitsschub, wenn innerhalb von einem Jahr zwei oder mehr Kortikoidstoßtherapien benötigt wurden, oder bei steroidabhängigem Verlauf eine Azathioprinmedikation begonnen werden. Die tägliche Dosis sollte bei $2-2,5 \mathrm{mg} / \mathrm{kg}$ KG pro Tag liegen. Alternativ kann 6-Mercaptopurin eingesetzt werden, die Dosis sollte zwischen $1-1,5 \mathrm{mg} / \mathrm{kg} \mathrm{KG} / \mathrm{d}$ betragen [251-253]. Der Einsatz von Thiopurinen in dieser Situation wird durch weitere Fallserien und kleinere Studie unterstützt [254-258].

Ob eine Weiterführung der 5-ASA-Therapie nach Beginn einer Therapie mit einem Immunsuppressivum (Azathioprin, 6-Mercaptopurin, Methotrexat) sinnvoll ist, wird kontrovers diskutiert. Ein zusätzlicher Nutzen bez. der Verhinderung von erneuten Schüben besteht nicht [259]. Allerdings kann die Fortsetzung der 5-ASA-Therapie unter dem Gesichtspunkt der Chemoprävention des kolorektalen Karzinoms sinnvoll sein (siehe Kapitel 8 „Karzinomprophylaxe“). Bei chronischer Krankheitsaktivität (Therapieresistenz/Steroidabhängigkeit) ist zunächst der Einsatz von Azathioprin/6-Mercaptopurin oder Methotrexat indiziert. Weitere Therapiealternativen stellen Infliximab, Ciclosporin A und Tacrolimus dar (Einzelheiten siehe oben).

Eine Weiterführung der Therapie mit Glukokortikoiden ist oft unumgänglich, in den meisten Situation wird man jedoch versuchen, Steroide zügig in der Dosis zu reduzieren.

Bei der Behandlung von Kindern und Jugendlichen gelten die gleichen Therapieprinzipien wie bei Erwachsenen. Führt bei schwerer Colitis ulcerosa die intravenöse Kortikosteroidmedikation nicht innerhalb von 4-7 Tagen zu einer klinischen Verbesserung, wird die Gabe von Infliximab ( $5 \mathrm{mg} / \mathrm{kg} \mathrm{KG}$ ) Ciclosporin A (initiial $2 \mathrm{mg} / \mathrm{kg} / \mathrm{d}$ als Infusion, nach Erreichen der
Remission $5-8 \mathrm{mg} / \mathrm{kg} / \mathrm{d}$ p.os in 2 Dosen) oder Tacrolimus $(0,2 \mathrm{mg} / \mathrm{kg} \mathrm{KG} / \mathrm{d}$ p.os in 2 Dosen) empfohlen. Die Dosisanpassung erfolgt bei beiden Medikamenten über Messungen der Talspiegel.

\section{Akuter Schub unter remissionserhaltender Therapie}

\section{Empfehlung 4.20}

Wird ein 5-ASA-freisetzendes Präparat zur Remissionserhaltung genutzt, soll im Falle eines akuten Schubes mit leichter bis mäßiger Aktivität zunächst die Dosierung auf $\geq 3 \mathrm{~g} / \mathrm{d}$ erhöht werden. [Evidenzgrad: B, Konsensusstärke: Konsens, Empfehlungsstärke: $\uparrow \uparrow]$

\section{Empfehlung 4.21}

Führt diese Maßnahme nicht zügig zu einer Besserung der Symptomatik, soll eine systemische Steroidtherapie initiiert werden. [Evidenzgrad: B, Konsensusstärke: Konsens, Empfehlungsstärke: $\uparrow \uparrow]$

\section{Empfehlung 4.22}

Wird Azathioprin oder 6-Mercaptopurin zur Remissionserhaltung eingesetzt, sollte eine systemische Steroidtherapie begonnen werden.

[Evidenzgrad: D, Konsensusstärke: Konsens, Empfehlungsstärke: $\uparrow \uparrow]$

\section{Erläuterung}

Mehrere Studien konnten zeigen, dass neuere galenische Formulierungen und hochdosierte 5-ASA-freisetzende Präparate eine Therapieverbesserung und Vereinfachung bringen können [191-193]. Hanauer et al. konnten 2007 zeigen, dass höherer Dosierungen $(4,8 \mathrm{~g} / \mathrm{d})$ gegenüber niedrigeren Dosierungen $(2,4 \mathrm{~g} / \mathrm{d})$ überlegen sind [260]. Eine weitere Studie konnte zeigen, dass der Wechsel von niedriger dosiertem oralem 5-ASA auf MMX-Mesalazin in einer Dosierung von $4,8 \mathrm{~g} / \mathrm{d}$ statistisch signifikant wirksamer ist in der Induktion einer Remission, als ein Wechsel auf MMX-Mesalazin in einer Dosierung von 2,4 g/d [212]. Somit scheint zunächst eine Dosiserhöhung auf mehr als $3 \mathrm{~g} / \mathrm{d}$ ein sinnvoller Weg zu sein. Kommt es trotz Dosisanpassung der 5-ASA-Therapie nicht zu einer Besserung der Symptomatik, muss eine Eskalation der Therapie hin zur Kortikosteroidtherapie in Erwägung gezogen werden. Wie schnell eine Eskalation zu erfolgen hat, muss vom Einzelfall und der Schwere der Symptomatik abhängig gemacht werden.

Wird Azathioprin oder 6-Mercaptopurion als remissionserhaltende Therapie eingesetzt, sollte bei akutem Schub zunächst eine systemische Steroidtherapie begonnen werden.

Die medikamentöse Therapie bei Kindern und Jugendlichen entspricht prinzipiell der bei erwachsenen Patienten.

\section{Komplikationen der schweren Colitis ulcerosa}

Das toxische Megakolon, Perforationen und Hämorrhagien stellen gefürchtete Komplikationen der schweren Colitis ulcerosa dar. Das toxische Megakolon ist definiert als eine segmentale oder ausgedehntere Dilatation des Kolons auf über $6 \mathrm{~cm}$ ohne verursachende Obstruktion und mit den Zeichen einer systemischen Krankheitsbeteiligung [261, 262]. Ursache ist in 
der Regel ein unkontrollierter Krankheitsverlauf (nicht erkannt, nicht oder unzureichend behandelt, therapierefraktär). Diese Komplikation tritt in etwa bei $5 \%$ der stationär behandelten Patienten auf. Sie bedarf der schnellen und intensiven Behandlung sowie einer engen Kooperation zwischen Gastroenterologen/Kindergastroenterologen und Chirurgen. Prinzipiell gleicht die Therapie der der schweren Colitis ulcerosa. Eine antibiotische Therapie sollte, um septische Komplikationen zu vermeiden, erwogen werden (z.B. Ceftriaxon/Metronidazol). Die häufigste schwere Komplikation der Colitis ulcerosa ist die schwere Blutung. Sie tritt in der Regel nach Entwicklung des toxischen Megakolons oder nach Koloskopie auf und ist mit einem wesentlichen Anstieg der Mortalität behaftet [261]. Eine retrospektive Analyse von Operationen bei CU in 158 Patienten ergab, dass $12 \%$ der Patienten wegen eines toxischen Megakolons, Perforation oder Blutung notfallmäßig operiert werden mussten [263].

\section{Schmerztherapie}

\section{Empfehlung 4.23}

Schmerzen können in allen Stadien der Erkrankung aus verschiedenen Ursachen auftreten. Eine differenzierte Schmerzanalyse (Krankheitsaktivität, Nebenwirkungen der antiinflammatorischen Therapie, funktionelle gastrointestinale Störungen, psychische Störungen) soll vor Einleitung einer symptomatischen Schmerztherapie durchgeführt werden.

[Evidenzgrad: D, Konsensusstärke: starker Konsens, Empfehlungsstärke: $\uparrow \uparrow$ ]

\section{Empfehlung 4.24}

Im akuten Schub und bei chronisch-aktiven Verläufen kann bei anhaltenden Bauchschmerzen trotz antiinflammatorischer Therapie eine symptomatische Schmerztherapie mit Metamizol oder ggf. Opioiden durchgeführt werden.

[Evidenzgrad: D, Konsensusstärke: starker Konsens, Empfehlungsstärke: $\uparrow]$

\section{Empfehlung 4.25}

Eine Dauertherapie mit Opioiden sollte vermieden werden. [Evidenzgrad: D, Konsensusstärke: starker Konsens, Empfehlungsstärke: $\downarrow]$

\section{Erläuterung}

Im Falle einer symptomatischen Schmerztherapie sind die Regeln der allgemeinen und speziellen Schmerztherapie $\mathrm{zu}$ beachten. Vor Einleitung einer symptomatischen Schmerztherapie ist auszuschließen, dass die Schmerzsymptomatik durch Optimierung der antiinflammatorischen Therapie, chirurgischen Therapie oder durch Absetzen von schmerzauslösenden Medikamenten behandelbar ist. Eine sorgfältige Analyse der Schmerzsymptomatik und ihrer biologischen, psychischen und sozialen Komponenten ist durchzuführen. Bei häufigen oder dauerhaften Schmerzen soll eine kontinuierliche orale Schmerztherapie mit retardierten Präparaten inklusive einer Bedarfsschmerzmedikation durchgeführt werden. Bei Opioiden sollen kurz wirksame Darreichungsformen (Tropfen, intravenös) wegen ihrer potenziell suchtfördernden Wirkung vermie- den werden. Die Gabe von Opioiden bei funktionellen gastrointestinalen Störungen oder psychischen Störungen als Ursache der Schmerzen ist kontraindiziert [264]. Zur symptomatischen Schmerztherapie der Colitis ulcerosa liegen keine kontrollierten Studien vor. Empfehlungen zur symptomatischen Schmerztherapie gründen sich daher auf klinischer Erfahrung und auf Extrapolationen von Therapieprinzipien anderer chronischer nicht karzinombedingter Schmerzsyndrome [265]. Bezüglich des Einsatzes von Paracetamol besteht bez. einer möglichen Induktion eines Krankheitsschubs eine widersprüchliche Datenlage, wobei sehr selten bei hochnormalen Dosen eine schwere Hepatotoxizität auftreten kann [16, 266]. Bei der Therapie mit Opioiden sind relative Kontraindikationen (z.B. Ileussymptomatik, toxisches Megakolon) sowie Nebenwirkungen (Sedierung, missbräuchliche Verwendung) zu beachten. Bei Patienten mit psychischer Komorbidität besteht ein erhöhtes Risiko missbräuchlicher Verwendung von Opioiden [266268]. In der Akutschmerztherapie bei fulminanten Verläufen sind Opioide relativ kontraindiziert. Ketamin kann zur Schmerztherapie bei fulminanten Verläufen verwendet werden [269].

\section{Medikamentöse Therapie \\ Aminosalicylate}

Es steht eine Reihe unterschiedlicher Präparate mit unterschiedlicher Galenik zur Verfügung: Azo-Verbindungen, kontrolliert freisetzende Präparate, $\mathrm{pH}$-abhängig freisetzende Präparate und Kombinationen der unterschiedlichen Verfahren. Im klinischen Alltag besteht derzeit für kein Präparat bei vergleichbarer Dosierung ein Vorteil hinsichtlich der klinischen Wirksamkeit. Es liegen zahlreiche Metaanalysen vor, die belegen, dass Aminosalicylate in der Therapie sicher und wirksam sind [190, 270-272]. 5-ASA und Sulfasalazin unterscheiden sich in ihrer Wirksamkeit bez. der Remissionsinduktion nicht, allerdings scheint 5-ASA besser verträglich zu sein als SASPPräparate. Nebenwirkungen der 5-ASA-Therapie werden bei ca. $15 \%$ der Patienten beobachtet. Die häufigsten sind Diarrhöen, Kopfschmerzen, Übelkeit, Erythem und Thrombozytopenie (in absteigender Häufigkeit). Eine Metaanalyse zeigte, dass die Häufigkeit der Nebenwirkungen unter Placebo- und 5-ASATherapie gleich sind [3]. Renale Nebenwirkungen sind selten und eher mit der Schwere der Erkrankung assoziiert als mit der 5-ASA-Gabe [273].

\section{Kortikosteroide}

Die Wirksamkeit der Kortikosteroide wurde in 2 kontrollierten Studien vor über 50 Jahren belegt $[106,216]$. In einer Studie, die die Wirksamkeit in neudiagnostizierten Colitis-ulcerosaFällen untersuchte, zeigte, dass nach 30 Tagen 51\% eine komplette Remission und 31\% eine inkomplette Remission hatten [20]. Das erhebliche Nebenwirkungsprofil von Kortikosteroiden verbietet deren dauerhafte Anwendung in höheren Dosierungen.

\section{Infliximab}

Eine systematische Übersichtsarbeit konnte die Wirksamkeit von Infliximab in der Therapie der mäßig schweren bis schweren Colitis ulcerosa mit steroidrefraktärem Verlauf und/oder fehlender Wirkung einer begleitenden Immunmodulatortherapie zeigen [224]. Die einzelnen Studien und ihre jeweilige Anwendbarkeit wurden in den entsprechend Abschnitten besprochen. Es muss hier darauf hingewiesen werden, dass trotz der 
8-wöchigen Gabe von $5 \mathrm{mg} / \mathrm{kg} \mathrm{KG} / \mathrm{d}$ Infliximab nur 21\% der Patienten nach 7 Monaten und 26\% nach 12 Monaten in steroidfreier Remission sind. In den Studien zur Anwendung von Infliximab in der Therapie der Colitis ulcerosa stellte sich der Wirkstoff als sicher bei der indizierten Anwendung dar. Die Kontraindikationen sind streng zu beachten. Es besteht ein besonderes Risiko für Infektionserkrankungen und demyelinisierende Erkrankungen. In einer kombinierten Analyse von 484 Patienten der ACT-Studien wurden 8 Pneumonien, eine Tuberkulose, eine Histoplasmose und 4 neoplastische Erkrankungen identifiziert sowie weitere 3 Neuropathien. Ob die Entwicklung maligner Erkrankungen (insbesondere von Lymphomen) durch Infliximab begünstigt wird oder ob die beobachtete geringfügige Erhöhung des Risikos einer solchen Erkrankung von anderen Faktoren abhängt, ist nicht definitiv geklärt [200, 274-276]. Insgesamt bestätigen die vorliegenden Daten die Sicherheit der Anwendung von Infliximab bei Berücksichtigung von Indikationen/Kontraindikationen und nach Abwägung von Risiken und potenziellem Nutzen.

\section{Thiopurine}

Die Datenlage zur Wirksamkeit der Thiopurine in der Behandlung der akuten Colitis ulcerosa sind begrenzt. Die Therapieprinzipien entsprechen denen in der Behandlung des Morbus Crohn.

Eine körpergewichtsadaptierte Dosierung von $2-2,5 \mathrm{mg} / \mathrm{kg} \mathrm{KG}$ tgl. hat gegenüber einer 6-TGN (aktive Metaboliten des Azathioprins)-Spiegel-adaptierten Dosierung keine Nachteile [277]. Es wird kontrovers diskutiert, ob von Beginn an direkt die volle Dosis gegeben werden kann oder langsam eine Dosissteigerung (innerhalb von 2-4 Wochen) bis zur therapeutischen Dosis durchgeführt werden sollte. Bei Übelkeit, Erbrechen und Magendruck unter der Azathioprintherapie ist ein Therapieversuch mit 6-Mercaptopurin gerechtfertigt. Bei 3-6\% der Patienten tritt unter Azathioprineinnahme eine Leberwerterhöhung auf. In diesen Fällen lässt sich bei der Hälfte die Therapie nach einem Wechsel auf 6-Mercaptopurin fortführen. Bei etwa 3\% tritt eine Pankreatitis auf. In diesen Fällen ist ein Wechsel auf 6-Mercaptopurin nicht sinnvoll. Die Therapie mit einer der beiden Substanzen sollte über einen längeren Zeitraum erfolgen. Vermutlich ist auch noch nach 4 Jahren ein positiver Effekt der Substanzen zu verzeichnen [278-280]. Beide Substanzen werden über die Thiopurin-Methyltransferase (TPMT) abgebaut. Bei einer verminderten Aktivität der TPMT tritt unter Therapie mit Azathioprin häufig im Verlauf eine schwere Leukopenie auf [281, 282]. Dies ist insbesondere der Fall bei einer homozygoten Mutation im TPMT-Gen, die bei ca. 0,3\% der Bevölkerung auftritt. Die Mehrzahl der Leukopenien unter Azathioprin/6-Mercaptopurin-Therapie (etwa $80 \%$ ) tritt jedoch unabhängig vom TPMT-Genotyp auf [281]. Daher ist eine engmaschige Blutbildkontrolle unter der Therapie notwendig. Diese sollte während der ersten 2 Monate häufiger und dann weiterhin in regelmäßigen Abständen durchgeführt werden. Unter der Therapie mit Azathioprin tritt eine Lymphopenie auf, die therapeutisch erwünscht ist. In der Kombinationstherapie mit anderen Immunsuppressiva kann diese Lymphopenie so ausgeprägt sein, dass die T-Helferzellzahl unter $200 / \mu l$ absinkt. Es ist offensichtlich, dass hierunter eine Immundefizienz entstehen muss. Die Gesamtleukozytenzahl ist hier nicht aussagekräftig, da diese durch einen hohen Neutrophilenanteil noch im Normbereich sein kann, wenn schon eine ausgeprägte Lymphopenie vorhanden ist. Daher sollte gelegentlich ein Differenzialblutbild erhoben werden, welches eine Abschätzung der T-Helferzellzahl erlaubt.

\section{Calcineurininhibitoren}

Anwendung und Dosierung von Ciclosporin A und Tacrolimus werden in den Abschnitten „schwere CU, beliebige Ausdehnung“ und „CU, refraktär auf systemische Steroide“ besprochen. Die Therapie sollte solange fortgeführt werden bis ein sonstiger Immunmodulator (z.B. Azathioprin) in die Therapie aufgenommen und wirksam sein kann (in der Regel 3-6 Monate). Trotz dieses Vorgehens ist mit einem hohen Anteil von erneuten Schüben innerhalb des folgenden Jahres zu rechnen [240, 244, 283]. Als Nebenwirkungen der Therapie treten arterielle Hypertonie, Parästhesien, Tremor und Kopfschmerzen am häufigsten auf. Bei etwa der Hälfte der Patienten treten Hypomagnesämie, Nierenschädigungen oder gastrointestinale Nebenwirkungen auf [234]. Die Häufigkeit der Nebenwirkungen ist dosisabhängig. Calcineurininhibitoren können einen Diabetes mellitus auslösen [284]. Auf die gegebenenfalls durchzuführende Chemoprophylaxe gegen opportunistische Infektionen wird in Kapitel 6 eingegangen.

\section{Methotrexat}

Eine randomisierte, placebokontrollierte Studie mit 67 Patienten mit aktiver Colitis ulcerosa, die bereits Kortikosteroide und/oder Immunsuppressiva erhalten hatten, untersuchte die Wirksamkeit von oralem Methotrexat $12,5 \mathrm{mg}$ einmal wöchentlich und konnte keinen Vorteil gegenüber Placebo zeigen [285]. Eine weitere Studie untersuchte den Nutzen von MTX in der Therapie von steroidabhängigen Verläufen und verglich die Anwendung von 6-MP (1,5 mg/kg KG/Tag), MTX (15 mg/Woche) und 5-ASA (3g/d). Die Remissionsraten in Woche 30 lagen in der MTXGruppe bei 58,3\%, wobei kein signifikanter Unterschied zur 5ASA-Gruppe (25\%) festgestellt werden konnte [286]. Die Dosierungen lagen in den aufgeführten Studien mit 12,5 und $15 \mathrm{mg} /$ Woche in einem niedrigen Bereich, sodass möglicherweise bei einer höheren Dosierung höhere Ansprechraten erreicht werden. Der Wert von MTX als Zweitlinientherapie nach der Anwendung von Thiopurinen bei Colitis ulcerosa bleibt insgesamt aufgrund der vorliegenden Daten umstritten.

\section{Medikamentöse Behandlungsoptionen mit aktuell unklarer Datenlage}

Für eine Reihe weiterer Behandlungsoptionen können aufgrund der aktuellen Datenlage derzeit keine Empfehlungen abgegeben werden. Für einige dieser Behandlungsoptionen werden derzeit klinische Untersuchungen durchgeführt bzw. stehen vor dem Abschluss oder befinden sich in der Auswertungsphase. Es werden hier zumindest für einige Verfahren weitere relevante Evidenzen erwartet (z.B. Adalimumab, Leukozytenapherese). So zeigen erste Ergebnisse, dass Adalimumab bei Patienten mit Colitis ulcerosa im Vergleich zu einer Placebobehandlung eine signifikante Remission induzieren und auch erhalten kann. Für andere Substanzen wie Basiliximab und Visilizumab konnte in den vorliegenden Studien keine signifikante klinische Remissionsinduktion oder -erhaltung im Vergleich zu Placebo nachgewiesen werden. Für diese Behandlungsoptionen und weitere Behandlungsmöglichkeiten wie $z$. B. Interferon- $\beta$, anti-Adhäsionsmolekül-Therapien, weite- 
re anti-TNF-Strategien und Nikotin können derzeit aufgrund der unzureichenden Datenlage keine Therapieempfehlungen ausgesprochen werden.

\section{Kapitel 5: Remissionserhaltung}$$
\text { Empfehlung } 5.1
$$

Definition: Die Remission der CU kann klinisch und endoskopisch definiert werden. Kriterien der klinischen Remission sind Abwesenheit von Diarrhö ( $\leq 3$ ungeformte Stühle/d), kein sichtbares Blut im Stuhl und keine durch die CU bedingten intestinalen oder extraintestinalen Beschwerden. Die endoskopische Remission wird durch das Fehlen entzündlicher Veränderungen bestimmt. [Evidenzgrad: D, Konsensusstärke: starker Konsens, Empfehlungsstärke: $\uparrow$ ]

\section{Erläuterung}

Von den verschiedenen Kriterien „Klinik“, „Morphologie“ oder „Labor“ ist das klinische Kriterium als einziges in allen wissenschaftlichen Untersuchungen, die die Basis für Therapieentscheidungen bilden, enthalten. Die meisten klinischen Studien basieren auf dem auch als „Mayo Score“ bezeichneten Index [287, 288]. Der Mayo Score berücksichtigt gleichberechtigt die Frequenz durchfälliger Stühle, die Gesamtbeurteilung durch den Arzt, der Anteil blutiger Stühle und das Vorhandensein sigmoidoskopischer entzündlicher Veränderungen, wobei nur die beiden letzten Parameter als objektiv angesehen werden. Keine Einigkeit besteht in der Wahl eines bestimmten Aktivitätsindex zur Definition der Remission oder Definitionen basierend auf Laborwerten. Eine Definition der Remission aufgrund einer Rektoskopie/Sigmoidoskopie oder Histologie oder Sonografie allein wird im Allgemeinen nicht akzeptiert. Es gibt jedoch Hinweise dafür, dass das zusätzliche Vorhandensein einer vollständigen endoskopischen und histologischen Remission auch mit einer stabileren (d.h. länger dauernden) klinischen Remission einhergeht [103, 289-291].

\section{Empfehlung 5.2*}

Bei Colitis ulcerosa soll eine remissionserhaltende Therapie (oral oder rektal) mit Aminosalizylaten erfolgen.

[Evidenzgrad: A, Konsensusstärke: starker Konsens, Empfehlungsstärke: $\uparrow \uparrow$ ]

\section{Erläuterung}

Die Evidenz für den Einsatz von 5-Aminosalizylaten zur Remissionserhaltung bei Colitis ulcerosa ist sehr gut. Es existiert eine Reihe von Metaanalysen zu diesem Thema [292-295]. Bisher gibt es keinen wissenschaftlichen Beleg für eine bessere Therapie in dieser Situation. Nicht geeignet zur Remissionserhaltung sind systemische Steroide, Diät und Quellmittel. Obwohl mittlerweise in drei kontrollierten Studien als effizient belegt, findet der primäre Einsatz von E. coli Nissle aufgrund der im Vergleich zu Aminosalizylaten weniger umfangreichen Studienlage keine einheitliche Bewertung.

\section{Empfehlung 5.3}

Angesichts des Nebenwirkungsprofils bei nicht unterschiedlicher Effizienz soll 5-ASA der Vorzug gegenüber SASP gegeben werden. [Evidenzgrad: A, Konsensusstärke: starker Konsens, Empfehlungsstärke: $\uparrow \uparrow$ ]

\section{Erläuterung}

Bei den vorliegenden Studien wurde eine selektierte Population untersucht, die nur eine Extrapolation auf unselektierte Patienten zulässt. In einer Metaanalyse [293] wird auf eine potenzielle Überlegenheit von Sulfasalazin hingewiesen, wenn alle anderen 5-ASA-Präparate dagegen verglichen werden. Allerdings wurden in den berücksichtigten Studien nur Sulfasalzin-tolerante Patienten eingeschlossen, sodass ein adäquater Vergleich beider Substanzen nicht möglich ist. In einer aktuellen Metaanalyse wurden zwanzig randomisierte, placebokontrollierte Studien eingeschlossen [296]. Der Vergleich von Sulfasalazin mit 5-ASA zeigte keinen signifikanten Unterschied (RR für den Rückfall 0,98 [95\% CI0,78-1,23], p=0,85), bei einer Tendenz zu mehr Nebenwirkungen (RR 0,76, 95\% $\mathrm{CI} 0,54-1,07, \mathrm{p}=0,11$ ) [296]. Insgesamt ist von einer geringeren Nebenwirkungsrate einer 5-ASA Therapie gegenüber der Verwendung von Sulfasalazin auszugehen [297, 298].

\section{Empfehlung 5.4*}

Der Weg der Applikation soll sich nach dem Befallsmuster der Erkrankung richten. Die Proktitis und die distale Kolitis sollen primär topisch therapiert werden.

[Evidenzgrad: A, Konsensusstärke: Konsens, Empfehlungsstärke: $\uparrow \uparrow$ ]

\section{Erläuterung}

Die orale Anwendung wurde für alle Präparate untersucht, die rektale Anwendung nur in eingeschränkter Weise und in den verschiedenen Formulierungen (Suppositorien, Rektalschaum, Klysma) nur für 5-ASA. Bei der distalen CU gibt es Hinweise auf eine therapeutische Überlegenheit der rektalen Applikation [183] insbesondere gegenüber Steroiden [299]. Die Kombination aus oraler und rektaler Therapie ist der oralen Monotherapie überlegen [300]. In dieser Kombination kann die topische Therapie durchaus intermittierend angewandt werden [301].

\section{Empfehlung 5.5}

Es sollen Dosen verwendet werden, für die in Studien Wirksamkeit nachgewiesen wurde ( $\bullet$ Tab. 9).

[Evidenzgrad: B, Konsensusstärke: starker Konsens, Empfehlungsstärke: $\uparrow \uparrow$ ]

\section{Erläuterung}

Es liegen insgesamt nur wenige Dosisfindungsstudien vor, die teils kontroverse Ergebnisse zeigen [293, 298, 311, 312]. Überwiegend handelt es sich in diesen Studien um einen Vergleich zweier verschiedener Dosierungen, während es kaum Untersuchungen mit mehr als zwei Dosierungen gibt [313]. Eine Metaanalyse konnte keinen Trend für eine Dosisbeziehung von Aminosalizylaten in der Remissionserhaltung erkennen [293]. Insgesamt muss jedoch festgehalten werden, dass die Dosiswirkungskurve nicht nach oben abgeschlossen ist. Somit sind die allgemein verwendeten Dosen nicht notwendigerweise op- 
Tab.9 Minimale Dosierung von 5-ASA-Präparaten mit belegter Wirksamkeit. oral:

\begin{tabular}{|c|c|c|}
\hline Formulierung & Dosis & Referenz \\
\hline $\begin{array}{l}\text { - 5-ASA als slow oder delayed release } \\
\text { Formulierung }\end{array}$ & $1,5 \mathrm{~g} / \mathrm{d}$ & [302] \\
\hline - 5-ASA in MMX-Formulierung & $2,4 \mathrm{~g} / \mathrm{d}$ & {$[191,303]$} \\
\hline - Olsalazin & $1,0 \mathrm{~g} / \mathrm{d}$ & [304] \\
\hline - Sulfasalazin & $2 \mathrm{~g} / \mathrm{d}$ & [305] \\
\hline \multicolumn{3}{|l|}{ rektal: } \\
\hline bei Linksseitenkolitis: & 5-ASA-Klysmen & \\
\hline$-1 \mathrm{~g} / \mathrm{d}$ & & [306] \\
\hline - $4 \mathrm{~g}$ jeden 3. Tag & & [307] \\
\hline $\begin{array}{l}-4 \mathrm{~g} / \mathrm{d} \text { jeweils an den ersten } 7 \text { Tagen des } \\
\text { Monats }\end{array}$ & & [308] \\
\hline \multicolumn{3}{|l|}{ bei Proktitis: 5-ASA-Suppositorien } \\
\hline$-2 \times 500 \mathrm{mg} / \mathrm{d}$ & & {$[294,309]$} \\
\hline - $1 \mathrm{~g} / \mathrm{d}$ 3-mal die Woche & & {$[310]$} \\
\hline
\end{tabular}

timal. Da einzelne Patienten besser Mesalazin-Schaumpräparate als Klysmen vertragen, kann diese Darreichungsform vermutlich in analoger Dosierung verabreicht werden, auch wenn Studien zur Remissionserhaltung mit Schaumpräparaten nicht vorliegen.

\section{Empfehlung 5.6*}

Einzelne Präparate können als tägliche Einmalgabe gegeben werden.

[Evidenzgrad: A, Konsensusstärke: starker Konsens, Empfehlungsstärke: $\uparrow$ ]

\section{Erläuterung}

Die Aufteilung der Gesamtdosis auf 3 oder 2 Einzeldosen scheint zumindest für die aktive Entzündung nicht mehr notwendig zu sein [193] oder der Einmalgabe sogar unterlegen zu sein [314]. Ob dies auch für die Remissionserhaltung gilt, wurde in diesen Studien nicht untersucht, darf aber angenommen werden. Für die MMX-Präparation wurde eine Gleichwertigkeit der Einmalgabe für die Remissionerhaltung bereits belegt [311, 315]. Ob die Ergebnisse einen pharmakologischen Effekt oder eine verbesserte Compliance der Patienten widerspiegeln, ist dabei unklar, letztendlich aber auch nicht bedeutsam.

\section{Empfehlung 5.7}

Nach Erreichen der Remission soll die remissionserhaltende Therapie mit Aminosalizylaten mindestens 2 Jahre durchgeführt werden.

[Evidenzgrad: B, Konsensusstärke: starker Konsens, Empfehlungsstärke: $\uparrow \uparrow$ ]

\section{Erläuterung}

Zur Frage, ob und wann eine remissionserhaltende Dauertherapie einer nur beobachtenden Haltung überlegen ist, gibt es wenige wissenschaftliche Daten. Eine kontrollierte Studie zeigte, dass bei Patienten mit einer Remissionsdauer von bis zu 2 Jahren eine 12-monatige Gabe von 5-ASA das Auftreten von Rezidiven im Vergleich zu Placebo vermindern kann [316, 317]. Aus methodischen und statistischen Gründen (notwendige Gruppengrößen für volle statistische Aussagekraft) kann man die Ergebnisse dieser Untersuchung jedoch nicht als definitive Aussage zum Stellenwert einer remissionserhaltenden 5ASA-Therapie bei Patienten mit über 2-jähriger Remission werten. Die Möglichkeit einer interstitiellen Nephritis bei langjähriger Gabe von 5-ASA ist zu bedenken [318]. Das Risiko für eine interstitielle Nephritis scheint mit der 5-ASA-Dosis und Therapiedauer zu korrelieren [318]. Ein Problem aller bisher durchgeführten Studien zum Langzeiteffekt von 5-ASA ist sicherlich, dass die Therapie-Adhärenz kaum überprüft wurde.

\section{Statement 5.8}

Definition: Ein Versagen der remissionserhaltenden Therapie ist dann gegeben, wenn ein Schub trotz einer geeigneten remissionserhaltenden Therapie auftritt und eine Schubtherapie erfordert. Eine erneute, remissionserhaltende Therapie setzt zuerst eine Remissionsinduktion durch eine Schubtherapie voraus.

[Evidenzgrad: D, Konsensusstärke: starker Konsens]

\section{Erläuterung}

Wegen fehlender Studien kann kein gesichertes Stufenschema zur Intensivierung der remissionserhaltenden Behandlung formuliert werden. Lediglich einzelne Therapieformen sind miteinander verglichen worden. So ist eine kombinierte Therapie mit oral und zusätzlich rektal verabreichtem 5-ASA einer nur oralen 5-ASA-Therapie in Standarddosis in der Remissionserhaltung überlegen [319]. Eine orale Immunsuppression mittels Azathioprin/6-Mercaptopurin [320] kann ebenso wie die systemische Gabe von Infliximab [321, 322] die Remission erhalten, wobei weder ein guter Vergleich zur 5-ASA-Standardtherapie noch eine aussagekräftige Dosisfindungsstudie existiert. Der Erhalt einer Cyclosporin-A-induzierten Remission ist schwierig: In einem hohen Prozentsatz werden die Patienten innerhalb von 6 Monaten kolektomiert [323], es gibt aber auch Hinweise auf einen erfolgreichen Einsatz von Azathioprin als Sequenztherapie zum Remissionserhalt [324-327]. Auch liegen keinerlei Vergleiche für die Effektivität von medikamentöser und operativer Behandlung vor. Unter Berücksichtigung dieser sehr beschränkten Datenlage werden hier Vorschläge zur Intensivierung der remissionserhaltenden Therapie gemacht (Reihenfolge entsprechend dem Grad der Intensivierung).

\section{Empfehlung 5.9}

Bei häufigen oder schweren Schüben soll die remissionserhaltende Therapie eskaliert werden.

[Evidenzgrad: C, Konsensusstärke: starker Konsens, Empfehlungsstärke: $\uparrow \uparrow$ ]

\section{Erläuterung}

Wenn Schübe frühzeitig nach Erreichen einer Remission auftreten oder öfter als einmal pro Jahr unter einer adäquaten Therapie mit 5-ASA in einer etablierten Dosis, sollte die remissionserhaltende Therapie modifiziert werden. $\mathrm{Zu}$ dieser Thematik liegen bei CU nicht für alle Situationen eindeutigen Studiendaten vor. Es kann aber z.T. aus Studien zum Morbus Crohn abgeleitet werden, dass dieses Vorgehen auch bei CU gerechtfertigt ist (indirekte Anwendbarkeit). 


\section{Empfehlung 5.10*}

Möglichkeiten zur stufenweisen Therapieeskalation sind eine oral/ rektale Kombinationstherapie mit Aminosalizylaten, eine Erhöhung der oralen Dosis von 5-ASA, eine Therapie mit Azathioprin/ 6-Mercaptopurin oder Infliximab.

[Evidenzgrad: A, Konsensusstärke: Konsens, Empfehlungsstärke: $\uparrow \uparrow]$

\section{Empfehlung 5.11}

Methotrexat und Tacrolimus sollten zur Remissionserhaltung der CU eher nicht eingesetzt werden.

[Evidenzgrad: B, Konsensusstärke: Konsens, Empfehlungsstärke: $\downarrow]$

\section{Empfehlung 5.12}

Kortikosteroide sollen zur Remissionserhaltung nicht eingesetzt werden.

[Evidenzgrad: A, Konsensusstärke: starker Konsens, Empfehlungsstärke: $\uparrow \uparrow]$

\section{Erläuterung}

Die verstärkte Wirkung einer oral/rektalen Kombinationstherapie mit 5-ASA ist durch Studien gut belegt, falls bisher nur eine Monotherapie erfolgte [300]. Die Erhöhung der remissionserhaltenden Dosis wurde durch eine randomisierte, kontrollierten Studie sowie durch eine Metaanalyse gezeigt [294, 328]. Eine optimale Dosis für Azathioprin wurde bei der Indikation CU durch Studien nicht festgelegt. Es ist gibt jedoch klare Hinweise, dass Azathioprin und 6-Mercaptopurin effektiv sind [253, 320, 329-332]. Als Dosierung für Azathioprin wurden 1,5-2,5 mg/kgKG/d bzw. die Hälfte der Dosis für 6-Mercaptopurin beschrieben [320]. Aufgrund der pharmakodynamischen Eigenschaften der Substanz (Wirkungseintritt erst nach 6-12 Wochen) ist eine längerfristige Therapieplanung erforderlich. Die Wirksamkeit von Infliximab wurde ohne Unterschiede zwischen einer Dosierung von 5 oder $10 \mathrm{mg} / \mathrm{kg}$ KG alle 8 Wochen belegt (nach einer Induktionstherapie mit $5 \mathrm{mg} / \mathrm{kg} \mathrm{KG} \mathrm{im}$ 0, 2, 6 Wochen Schema) [321, 322, 333].

Bisher liegen keine ausreichenden Studiendaten vor, die einen Einsatz von Methotrexat oder Tacrolimus zur Remissionserhaltung nahelegen würden [234, 334, 335]. Eine Studie randomisierte 30 Patienten zu Methotrexat und 37 zu Placebo. Methotrexat wurde oral in einer Dosis von $12,5 \mathrm{mg} /$ Woche gegeben. 64\% der Patienten in der Methotrexatgruppe und 44\% der Patienten in der Plazebogruppe erlitten in der Beobachtungszeit von 9 Monaten einen Rückfall [334].

Für die Wirksamkeit einer Remissionserhaltungstherapie durch Kortikosteroide gibt es sowohl in der lokalen Anwendung [336] als auch in der systemischen Applikation keine Evidenz [337].

\section{Empfehlung 5.13}

Die medikamentösen Therapiemöglichkeiten und -risiken sollen gegen eine Operation abgewogen werden.

[Evidenzgrad: B, Konsensusstärke: Konsens, Empfehlungsstärke: $\uparrow \uparrow]$

\section{Erläuterung}

Wenn Nutzen und Risiken einer medikamentösen Therapie überdacht werden, sollte immer die Möglichkeit einer restaurativen Kolektomie mit Pouchanlage in Betracht gezogen werden. Auch die Unzufriedenheit des Patienten mit der medikamentösen Dauertherapie kann eine Indikation für eine Kolektomie darstellen.

\section{Empfehlung 5.14}

Bei Steroidabhängigkeit soll primär Azathioprin/6-MP eingesetzt werden.

[Evidenzgrad: A, Konsensusstärke: starker Konsens, Empfehlungsstärke: $\uparrow \uparrow$ ]

\section{Erläuterung}

Die Evidenz für die Wirksamkeit einer remissionserhaltenden Therapie mit Azathioprin ist in der Zwischenzeit sehr gut [253, 320, 329-332]. Da die Raten für den steroidfreien Remissionserhalt klar höher liegen als für Infliximab, sollte Azathioprin/ 6-Mercaptopurin zunächst eingesetzt werden.

\section{Empfehlung 5.15*}

Bei Unverträglichkeit von Aminosalicylaten kann der apathogene Escherichia coli Stamm Nissle 1917 (A) oder bei Kindern das probiotische Präparat VSL\#3 (B) eingesetzt werden. Empfehlungsstärke: siehe Empfehlungstext, Konsensusstärke: starker Konsens, Empfehlungsstärke: $\uparrow$ ]

\section{Erläuterung}

Es sind 3 kontrollierte Studien erschienen, die die Wirksamkeit von Escherichia coli Stamm Nissle 1917 in einer Dosierung von $2 \times 100 \mathrm{mg} / \mathrm{d}$ in der Remissionserhaltung belegen [338340]. Auch bei Kindern scheint E. coli Nissle remissionserhaltend zu wirken [341]. Darüber hinaus wurde auch ein Wirknachweis für VSL\#3 bei Kindern geführt [342].

\section{Kapitel 6: Infektiologische Probleme}

Die Datenlage zu infektiologischen Problemen bzgl. Diagnostik und Therapieempfehlungen bei Patienten mit chronisch entzündlichen Darmerkrankungen ist limitiert, sodass die Statements und Empfehlungen häufig auf Analogien zu Daten aus anderen Fachgebieten (z.B. der Rheumatologie oder Transplantationsmedizin) basieren bzw. Expertenmeinungen darstellen. Besondere Erfahrungen in der Therapie infektiöser Probleme sind hilfreich, sodass in Abhängigkeit von der Komplexität des Krankheitsbilds infektiologische Kompetenz einzubeziehen ist. Diese Gesichtspunkte sind bei den einzelnen Punkten detailliert ausgeführt.

\section{Statement $6.1^{*}$}

Das Infektionsrisiko unter einer Therapie mit immunsuppressiven Medikamenten, einschließlich Kortikosteroiden, ist erhöht.

[Evidenzgrad: B, Konsensusstärke: starker Konsens] 


\section{Statement 6.2*}

Bei einer Kombinationstherapie aus mehreren immunsuppressiven Medikamenten steigt das Risiko mit Hinzunahme jedes weiteren immunsuppressiven Medikaments deutlich an. Ältere Patienten und Patienten mit Mangelernährung sind durch Infektionen besonders gefährdet.

[Evidenzgrad: C, Konsensusstärke: starker Konsens]

\section{Erläuterung}

Zahlreiche klinische und experimentelle Befunde weisen auf eine Fehlregulation des Immunsystems in der Pathogenese der CED hin [41]. Es gibt aber keine Hinweise, dass Patienten durch die Erkrankung selbst einen Immundefekt mit daraus resultierenden Infektionen entwickeln. Vielmehr kommt es im Rahmen der Therapie mit immunsuppressiven Medikamenten zu partiellen Defiziten des angeborenen und erworbenen Immunsystems und somit zu einer gesteigerten Suszeptibilität gegenüber Krankheitserregern. Bei schweren Krankheitsverläufen kann die daraus resultierende Mangelernährung die medikamentös-induzierte Immundefizienz verstärken. So haben Patienten mit einer Mangelernährung eine verminderte zelluläre Immunabwehr, Phagozytose und Zytokinproduktion sowie ein beeinträchtigtes Komplementsystem [343-345]. Darüber hinaus stellen das Alter der Patienten sowie vorliegende Begleiterkrankungen wie z.B. ein Diabetes mellitus weitere Risikofaktoren für Infektionen dar [22, 346, 347]. So scheinen Patienten mit einer CED, die älter als 50 Jahre sind, ein dreifach höheres Risiko zu haben, an einer opportunistischen Infektion zu erkranken als Patienten, die jünger als 25 Jahre alt sind (OR 3,0, $95 \%$ CI 1,2-7,2) [22]. Gründe für ein erhöhtes Infektionsrisiko im Alter sind Veränderungen sowohl im angeborenen als auch im erworbenen Immunsystem [346].

Derzeit werden zur Behandlung einer CED in der Regel folgende immunsuppressive Medikamente eingesetzt: Kortikosteroide, Azathioprin/6-Mercaptopurin, Methotrexat, Calcineurininhibitoren (CsA, Tacrolimus), TNF- $\alpha$-Antikörper und andere Biologika. Das Spektrum möglicher Infektionskrankheiten ist unter einer immunsuppressiven Therapie breiter, da auch Erreger, deren Pathogenität unter physiologischen Bedingungen gering ist („opportunistische Erreger“), unter diesen Umständen Infektionen hervorrufen können. Im Zusammenhang mit der Gabe von Immunsuppressiva wurden hierbei gehäuft sowohl bakterielle, virale und parasitäre Infektionen als auch Mykosen beschrieben [22, 348-353].

In der Diskussion über therapieinduzierte Infektionen wird häufig vergessen, dass die klassischen Medikamente für die Behandlung des Schubes, die Glukokortikoide, ebenso ein relevantes Infektionsrisiko beinhalten. So gibt es Daten aus der Rheumatologie, die zeigen, dass bei Patienten mit einer Tagesdosis von mehr als $10 \mathrm{mg}$ Prednisolon oder einer kumulativen Dosis von mehr als $700 \mathrm{mg}$ sowie einer Therapiedauer von mehr als zwei Wochen das Infektionsrisiko erhöht ist [354]. Eine Untersuchung von 497 Tuberkulose-Neuerkrankungen in Großbritannien zeigt, dass das Risiko, unter einer laufenden Kortikosteroidtherapie an einer aktiven Tuberkulose zu erkranken, im Vergleich zu Patienten ohne Kortikosteroidmedikation erhöht ist (OR 4,9, 95\% CI2,9-8,3). Dabei hatten Patienten mit einer maximalen Kortikosteroidtagesdosis von weniger als $15 \mathrm{mg}$ ein etwa 3-fach (OR 2,8, 95\% CI1,0 - 7,9), mit mehr als 15 mg ein nahezu 8-fach erhöhtes Risiko (OR 7,7, 95\% CI2,8 21,4) [355]. Mehrere Studien und Metaanalysen konnten au- ßerdem einen Zusammenhang zwischen einer präoperativen Kortikosteroidtherapie und einem, dosisabhängig, erhöhten postoperativen Infektionsrisiko und Wundheilungsstörungen nachweisen [356].

In einer Fallkontrollstudie an insgesamt 300 Patienten mit CED ist das Risiko einer Monotherapie mit Kortikosteroiden, Azathioprin/6-Mercaptopurin oder Infliximab, an einer opportunistischen Infektion zu erkranken, im Gegensatz zu Patienten ohne immunsuppressive Therapie jeweils um den Faktor 3 erhöht (OR 2,9, 95\% CI 1,5-5,3). Werden jedoch zwei oder drei der genannten Medikamente in Kombination angewendet, kommt es zu einem drastischen Anstieg des Infektionsrisiko (OR 14,5, 95\% CI4,9-43) [22]. In einer Kohortenstudie unter Einbeziehung von 10662 Patienten mit CED konnte gezeigt werden, dass sich die Inzidenz schwerer bakterieller Infektionen zwischen Patienten, die mit Infliximab behandelt wurden (8,9/ 1000 Patientenjahre, 95\% CI3,2-19,3) und Patienten, die entweder mit Kortikosteroiden, Thiopurinen oder Methotrexat behandelt wurden, nicht signifikant unterscheidet (6,8/1.000 Patientenjahre, 95\% CI4,5-9,9) ( $\mathrm{RR}=1,1,95 \% \mathrm{CI} 0,42-2,74)$ [357]. Bei retrospektiver Auswertung von Infliximab-naiven $(n=666)$ und Infliximab-behandelten $(n=743)$ Patienten mit CED zeigte sich kein Unterschied bez. der Infektionsraten zwischen den Gruppen. Eine gleichzeitige Behandlung mit Kortikosteroiden stellte jedoch in der Gruppe der mit Infliximab behandelten Patienten einen unabhängigen Risikofaktor für eine Infektion dar (OR 2,7, 95\% CI1,18-6,12). Interessanterweise fand sich dieser Zusammenhang für die Kombinationstherapie von Infliximab mit Azathioprin oder Methotrexat nicht [358]. Die Beobachtung, dass eine Kombinationstherapie mit Kortikosteroiden das Infektionsrisiko deutlich erhöht, ergibt sich ebenfalls bei der Auswertung des von der pharmazeutischen Industrie finanzierten TREAT-Registers. In diesem, aus über 6000 CED-Patienten bestehenden, Register konnte in der Multivariatanalyse kein Unterschied im Infektionsrisiko zwischen Patienten, die mit Infliximab behandelt wurden, und Infliximab-naiven Patienten gefunden werden (OR 0,99; 95\% CI0,64-1,54). Jedoch zeigte sich auch hier bei Patienten, die mit einer Kombinationstherapie aus Infliximab und Kortikosteroiden behandelt wurden, eine erhöhte Infektionsrate [359].

Auch wenn das Infektionsrisiko für alle Substanzklassen scheinbar in gleichem Maße erhöht ist, so gibt es doch Unterschiede bez. der Art der Infektionserkrankung unter einer spezifischen Therapie. So ist hinreichend bekannt, dass das Risiko einer granulomatösen Infektion (z.B. Tuberkulose, Listeriose) bei einer Therapie mit anti-TNF- $\alpha$-Antikörpern erhöht ist [360-362]. Bei einer Therapie mit AZA/6-MP ist tendenziell das Risiko einer viralen Infektion (z.B. Herpes zoster [OR 3,1, 95\% CI 1,7-5,6]) und bei einer Therapie mit Kortikosteroiden das Risiko für Pilzinfektionen (Candida spp.) erhöht - hier gibt es jedoch große Überschneidungen [22, 350, 363]. Im klinischen Alltag bei einer Infektion unter einer immunsuppressiven Therapie muss jedoch mit jedwedem infektiösen Erreger, auch parasitärer Genese, gerechnet werden.

Zusammenfassend lässt sich sagen, dass eine immunsuppressive Therapie, eine Malnutrition, das Alter des Patienten und Komorbiditäten das erhöhte Infektionsrisiko bei CED-Patienten bedingen. Hervorzuheben ist hierbei v. a. die Kombinationstherapie mit mehreren Immunsuppressiva, insbesondere wenn Kortikosteroide mitverwendet werden. Hier bedarf es einer engmaschigen Überwachung und Aufklärung der Patienten. 
Eine besondere Situation für Patienten mit Colitis ulcerosa ergibt sich in einer perioperativen Situation. Mehrere Studien konnten einen Zusammenhang zwischen einer präoperativen Kortikosteroidtherapie und einem, dosisabhängig erhöhten postoperativen Infektionsrisiko und Wundheilungsstörungen nachweisen [364 - 366]. Eine erst kürzlich erschienene Metaanalyse aus insgesamt 11 Studien kam zu dem Ergebnis, dass Patienten mit einer präoperativen Kortikosteroiddosis von mehr als $40 \mathrm{mg}$ am Tag ein zweifach erhöhtes Risiko für postoperative Komplikationen aufweisen (OR 2,04, 95\% CI 1,28 - 3,26). Bei der Auswertung des postoperativen Infektionsrisikos konnten hingegen nur Daten aus zwei Studien ausgewertet werden. Hier zeigte sich für Patienten mit einer präoperativen Kortikosteroidtagesdosis von mehr als $20 \mathrm{mg}$ ein erhöhtes postoperatives Infektionsrisiko (OR 1,17, 95\% CI0,73 - 1,87) [367]. Aufgrund der derzeitigen Datenlage sollte somit die präoperative Kortikosteroidtagesdosis weniger als $40 \mathrm{mg}$, idealerweise weniger als $20 \mathrm{mg}$ betragen. Für Azathioprin/6-Mercaptopurin hingegen ließ sich in mehreren Studien kein Hinweis für eine erhöhte Inzidenz von perioperativen Komplikationen, inklusive Infektionen, bei Patienten mit CED finden [368]. Ob es im Rahmen einer präoperativen Therapie mit Calcineurininhibitoren vermehrt zu perioperativen Infektionen kommt, kann aufgrund der begrenzten Datenlage zurzeit nicht beantwortet werden. Bezüglich des perioperativen Infektionsrisikos unter einer perioperativen Therapie mit Infliximab gibt es mehrere Studien, die jedoch zu unterschiedlichem Ergebnis kommen. Die in der Online-Version dargestellte Tabelle gibt eine Übersicht dieser Studien, die Patienten mit Morbus Crohn und Colitis ulcerosa einbeziehen. Bei der Betrachtung von 5 Studien mit insgesamt 706 Patienten konnten sie einen signifikanten Zusammenhang zwischen einer präoperativen Infliximabtherapie und einer erhöhten postoperativen Komplikationsrate nachweisen (OR 1,80, 95\% CI 1,12 - 2,87). Wurde die Genese der Komplikationen in einer Subgruppenanalyse weiter spezifiziert, ergab sich für postoperative infektiöse Komplikationen nach präoperativer Infliximabgabe zwar ein numerischer Unterschied, der jedoch statistisch nicht signifikant war (OR 2,24, 95\% CI0,63 - 7,95) [356].

\section{Empfehlung 6.3}

Bei Erstdiagnose bzw. spätestens vor Beginn einer immunsuppressiven Therapie sollte bei allen Patienten ein Infektionsscreening für Hepatitis B (D) und Tuberkulose (C) durchgeführt werden. Vor Einleitung einer Therapie mit anti-TNF- $\alpha$-Antikörpern ist das Tuberkulose-Screening zu aktualisieren.

[Evidenzgrad: s. Empfehlungstext, Konsensusstärke: Konsens, Empfehlungsstärke: $\uparrow$ ]

\section{Erläuterung}

Die Auswirkungen einer immunsuppressiven Therapie auf den Verlauf einer gleichzeitig bestehenden Hepatitis-B-Virus(HBV)Infektion bei CED-Patienten wurden nicht prospektiv erfasst. Erfahrungen aus anderen Fachdisziplinen zeigen eindrucksvoll, dass eine zytotoxische oder immunsuppressive Therapie, inklusive TNF- $\alpha$-Antikörpertherapie, bei HBV-Trägern das Risiko einer HBV-Reaktivierung signifikant erhöht. Die Inzidenz einer HBV-Reaktivierung während bzw. nach einer Chemotherapie beträgt bei HBs-Ag-Trägern $15-50 \%$ [377, 378]. Obwohl die HBV-Reaktivierung bei HBsAg-negativen Patienten deutlich seltener auftritt, wurde auch bei 14-20\% der anti-HBc- und anti-HBs-positiven Patienten mit Lymphomen unter Chemo- therapie eine Reaktivierung beschrieben [379]. In einer spanischen Studie an 80 Patienten mit Morbus Crohn, bei denen vor Therapiebeginn mit Infliximab ein Hepatitis-B-Screening durchgeführt wurde, fanden sich 3 Patienten mit einer chronischen HBV-Infektion. Zwei der Patienten entwickelten nach Beendigung der Infliximab-Therapie eine schwere Hepatitis, der dritte Patient verstarb aufgrund von Komplikationen einer Leberzirrhose [380]. Diese Beispiele zeigen, dass die Problematik der Reaktivierung nicht nur in den Langzeitfolgen (z.B. Zirrhose oder hepatozelluläres Karzinom), sondern auch das Risiko einer akuten Exazerbation der Hepatitis B mit fulminantem Verlauf erhöht ist [381]. Somit wird für alle Patienten, bei denen eine immunsuppressive Therapie eingeleitet werden soll, vor Beginn der Behandlung ein Screening auf HbsAg- und anti-HBc-Antikörper empfohlen. Bei seronegativen Patienten wird eine Impfung empfohlen (siehe Empfehlung 6.12). Eine medikamentös-präventive Therapie mit Nukleos(t)idanaloga wirkt der HBV-Reaktivierung entgegen und ist daher bei gefährdeten Patienten (HBsAg-positiv) indiziert. Bei HBs-Ag-negativen und anti-HBc-Antikörper-positiven Patienten wird eine engmaschige ALT- und HBV-DNA-Kontrolle empfohlen. Bezüglich der Dauer und Art der medikamentös-präventiven Therapie sowie dem Management von HBs-Ag-negativen und anti-HBc-Antikörper-positiven Patienten wird auf aktuelle Leitlinien verwiesen [382].

Die Durchführung eines Tuberkulose-Screenings bei der Erstdiagnose einer CED bzw. spätestens vor Beginn einer immunsuppressiven Therapie, dazu gehört auch - wie oben ausgeführt eine Glukokortikoidtherapie, soll die Möglichkeit eines falsch negativen Ergebnisses des Screeningverfahrens durch Immunsuppression verringern. So ist für den Tuberkulin-Hauttest (THT) gesichert, dass eine abgeschwächte Immunkompetenz ein falsch negatives Ergebnis bedingen kann [383]. Für Testverfahren, die auf dem Nachweis von IFN- $\gamma$ („Interferon-Gamma Release Assay“, IGRA) beruhen, wird die Gefahr eines falsch negativen Testergebnisses als geringer eingestuft, kann jedoch, v.a. bei Patienten mit schwerer Lymphopenie und Immunsuppression, ebenfalls nicht ausgeschlossen werden [384]. Aufgrund des erhöhten Tuberkulose-Risikos beim Einsatz von TNF- $\alpha$-Inhibitoren wird empfohlen, die Tuberkulose-Diagnostik vor Beginn einer Therapie mit TNF- $\alpha$-Inhibitoren zu aktualisieren, nachdem eine zwischenzeitliche Tuberkulose-Neuinfektion seit Erstdiagnose der CED und Tuberkulose-Screening nicht ausgeschlossen werden kann. Bezüglich der Durchführung des Tuberkulose-Screenings verweisen wir auf die Empfehlung 6.5.

\section{Empfehlung 6.4}

Die Entscheidung zum Screening auf HCV und HIV ist individuell abzuwägen.

[Evidenzgrad: D, Konsensusstärke: starker Konsens, Empfehlungsstärke: $\uparrow$ ]

\section{Erläuterung}

Die generelle Durchführung eines HCV-Screenings vor Einleitung einer immunsuppressiven Therapie wird nicht empfohlen. Durch eine immunsuppressive Therapie wird der Verlauf einer HCV-Erkrankung nicht maßgeblich negativ beeinflusst, ein positives Screening hätte somit keine Einflüsse auf die gegebene Therapieindikation. Erfahrungen aus der Transplantationsmedizin, Rheumatologie und Gastroenterologie zeigen, dass eine Therapie mit Kortikosteroiden, Azathioprin, Methotrexat und 
TNF- $\alpha$-Antikörper ohne Progredienz der HCV-Infektion durchgeführt werden kann [385-390].

Die generelle Durchführung eines HIV-Screenings vor Einleitung einer immunsuppressiven Therapie wird aufgrund der niedrigen HIV-Prävalenz in der Allgemeinbevölkerung in Deutschland ebenfalls nicht empfohlen. Bei Patienten, die einer Risikogruppe angehören, wird ein Screening vor Einleitung einer immunsuppressiven Therapie und in regelmäßigen Abständen unter laufender immunsuppressiver Therapie angeraten. So ist ein erhöhtes Infektionsrisiko bei HIV-positiven Patienten unter einer immunsuppressiven Therapie nicht ausgeschlossen. Weiterhin kann eine immunsuppressive Therapie negativen Einfluss auf die HI-Virus-Last haben. Bei positivem HIV-Test und geplanter immunsuppressiver Therapie wird die enge Zusammenarbeit und Therapieplanung mit einem HIVSpezialisten empfohlen.

\section{Empfehlung 6.5}

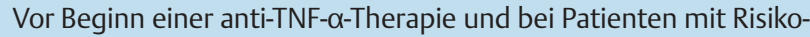
konstellationen, die immunsuppressiv behandelt werden sollen, soll per Anamnese plus Röntgen-Thorax plus immunologischen Testverfahren eine (latente) Tuberkulose ausgeschlossen werden. [Evidenzgrad: B, Konsensusstärke: starker Konsens, Empfehlungsstärke: $\uparrow \uparrow$ ]

\section{Erläuterung}

Grundsätzlich muss zwischen einer klinisch inapparenten Infektion und der aktiven, behandlungsbedürftigen Tuberkuloseerkrankung unterschieden werden [391]. Das Risiko, nach Erstinfektion mit Mykobacterium tuberculosis einmal im Laufe des Lebens an einer aktiven Tuberkulose zu erkranken, wurde aufgrund von Tuberkulin-Hauttest (THT)-basierten Reihenuntersuchungen auf maximal 10\% geschätzt, wobei zwischen Erstinfektion und Krankheitsausbruch Jahrzehnte liegen können [384]. Tierexperimentelle Studien haben die Bedeutung des TNF- $\alpha$ als Schlüsselkomponente in der Bildung der protektiven Granulomformation aufgezeigt [392].

Vor Einführung von Screeningmaßnahmen war die aktive TBC die häufigste opportunistische Infektionskrankheit bei einer Therapie mit anti-TNF- $\alpha$ Antikörper (144/100000 bei Infliximab behandelten Pat. vs. 27/100000 in der Allgemeinbevölkerung) [393]. Im Vergleich zur Allgemeinbevölkerung besteht ein bis zu 30-fach erhöhtes Risiko, unter einer Therapie mit anti-TNF- $\alpha$-Antikörpern an einer TBC zu erkranken [393-396]. Die Tuberkulose-Inzidenz in der Allgemeinbevölkerung hängt jedoch von vielen sozialen und individuellen Faktoren ab. So betrug die Tuberkulose-Inzidenz nach Angaben des Robert Koch-Instituts bei ausländischen Staatsbürgern 2008 in Deutschland mit 21/100000 das Fünffache der Inzidenz deutscher Staatsbürger (4/100000).

Neben dem erhöhten Risiko unter einer anti-TNF- $\alpha$-Therapie an einer TBC zu erkranken, ergibt sich auch für andere immunsuppressive Medikamente ein erhöhtes Erkrankungsrisiko. So konnten Abbera et al. für CED-Patienten noch vor Einführung der anti-TNF- $\alpha$-Antikörper-Therapie ein etwa 2-fach erhöhtes Tuberkulose-Risiko im Vergleich zur Normalbevölkerung ermitteln [397]. Wie oben dargestellt, ist auch bei einer „einfachen“ Glukokortikoidtherapie das Risiko, an einer Tuberkulose zu erkranken, erhöht.

Somit sollte vor dem Beginn einer immunsuppressiven Therapie, zwingend vor Gabe eines anti-TNF- $\alpha$-Antikörpers, das in- dividuelle Tuberkulose-Risiko des Patienten (Herkunftsland, Alter, Reise-, Umfeld- und Familienanamnese etc.) beachtet und ein Screening durchgeführt werden. Weiterhin muss unter einer laufenden immunsuppressiven Therapie, insbesondere bei einer anti-TNF- $\alpha$-Therapie, mit der erhöhten Gefahr einer Tuberkulose-Neuinfektion gerechnet werden. Mehr als 95\% der Tuberkulose-Fälle unter einer anti-TNF- $\alpha$-Therapie treten innerhalb der ersten 6 Monate auf [360, 393, 398]. Modellrechnungen zeigen, dass ein Großteil der Tuberkulose-Fälle, die erst ein Jahr nach Beginn der anti-TNF- $\alpha$-Therapie auftreten, auf eine Erstinfektion mit direktem Fortschreiten zur aktiven Tuberkulose zurückzuführen ist [399]. Bei dementsprechender Risikoanamnese (Auslandsreisen, Kontaktpersonen mit Tuberkulose, Arbeit im Gesundheitswesen etc.) muss somit auch unter laufender Therapie ein regelmäßiges TBC-Screening durchgeführt werden.

Der Nutzen eines Tuberkulose-Screenings vor Beginn einer anti-TNF- $\alpha$-Therapie, bestehend aus Anamnese, TuberkuloseHauttest, Röntgenthoraxbild und ggf. die Einleitung einer Therapie mit Isoniazid (INH) über 9 Monate, konnte durch das spanische BIOBADASER-Register belegt werden. Dieses Vorgehen führte zu einem Rückgang der Tbc-Infektionen um 74\% [400]. Es wurde jedoch auch über Fälle einer Tuberkulose-Reaktivierung unter anti-TNF- $\alpha$-Therapie trotz chemopräventiver Maßnahmen berichtet [401]. Unter einer Therapie mit antiTNF- $\alpha$-Antikörpern können die, oft mit der aktiven Tuberkulose einhergehenden, Symptome wie Fieber, Gewichtsverlust und Nachtschweiß fehlen [402]. Darüber hinaus besteht aufgrund der gehäuft extrapulmonalen Lokalisationen der Tuberkulose die Gefahr, Zeichen der Tuberkulose wie z.B. Diarrhö als Zeichen eines akuten CED-Schubs fehlzuinterpretieren [403, 404].

In den bisherigen deutschen Empfehlungen für das Screening von Patienten vor Beginn einer anti-TNF- $\alpha$-Therapie wurde neben einem aktuellen Röntgenthoraxbild und einer sorgfältigen Anamnese ein Tuberkulose-Hauttest gefordert. In den gemeinsamen Empfehlungen des Paul-Ehrlich-Instituts und des Deutschen Zentralkomitees zur Bekämpfung der Tuberkulose (DZK) wird für die Diagnosestellung einer latenten Tuberkuloseinfektion (LTBI) bei immunsupprimierten Patienten ein Indurationsdurchmesser größer $5 \mathrm{~mm}$ gefordert. Bei einer „schwachen“ Induration von weniger als $5 \mathrm{~mm}$ wird eine Testwiederholung angeraten [405]. Der Hauttest hat jedoch Nachteile. So führt eine Infektion mit nicht tuberkulösen Mykobakterien (NTM, Synonym: Umweltmykobakterien) aufgrund gemeinsamer Antigene der NTM mit Mycobacterium tuberculosis (MTB) ebenfalls zu einer zellulären Reaktion. Darüber hinaus können kreuzreaktive Antigene zu einem falsch positiven Ergebnis des Tuberkulose Hauttests nach vorangegangener Impfung mit M.bovis-BCG führen. Eine präventive Therapie einer latenten Tuberkuloseinfektion birgt jedoch die Gefahr z.T. schwerer Nebenwirkungen und Arzneimittelinteraktionen. Im Gegensatz hierzu kann ein falsch negatives Hauttest-Ergebnis Arzt und Patient in Sicherheit wiegen. Ursache für ein falsch negatives Ergebnis können u.a. Applikationsfehler oder eine abgeschwächte individuelle Immunkompetenz sein. Ein weiterer Nachteil des Tuberkulose-Hauttests besteht darin, dass er über keine positive Testkontrolle verfügt [406]. So liegen neben mehreren Fallberichten, in denen trotz späterer TuberkuloseErkrankung Patienten einen negativen Hauttest aufwiesen, inzwischen zahlreiche größere Studien vor, welche von einer hohen Rate an falsch negativen Tuberkulose-Hauttest-Resultaten 
bei Patienten mit einer rheumatoider Arthritis berichten [407, 408]. So analysierten Raval et al. die zwischen 2001 und 2006 bei der „U.S. Food and Drug Administration“ (FDA) eingegangenen Spontanmeldungen von Tuberkulose $(n=130)$ bei Patienten mit Infliximab-Therapie. Sie konnten zeigen, dass von den 67 Tuberkulose-Patienten, bei denen vor Beginn der Infliximab-Therapie ein Tuberkulose-Hauttest durchgeführt wurde, die Hälfte der Patienten $(n=34)$ einen negativen THT hatte [409]. In einer anderen Studie wurde bei 4 von 16 „Interferon- $\gamma$ Release Assay“(IGRA)-positiven Patienten mit Erkrankungen aus dem rheumatischen Formenkreis der gleichzeitig durchgeführte Tuberkulose-Hauttest als negativ beurteilt. Dabei war die fortlaufende Gabe von Prednisolon mit einer Tuberkulose-Hauttest-negativ-/IGRA-Test-positiv-Diskordanz assoziiert [410]. Diese Beobachtungen führten zu einer Aktualisierung der Empfehlungen der Deutschen Gesellschaft für Rheumatologie (DGRh) und des Zentralkomitees zur Bekämpfung der Tuberkulose [406]. Sie, wie auch andere ausländische Fachgesellschaften [411], favorisieren anstatt des TuberkuloseHauttests die Durchführung eines IGRAs. Diese Testverfahren beruhen auf dem Nachweis von IFN- $\gamma$, welches nach antigenspezifischer Stimulation durch T-Lymphozyten sezerniert wird. Die beiden in Deutschland kommerziell erhältlichen IGRATests basieren auf der direkten Messung der IFN- $\gamma$-Konzentration im Vollblut (QuantiFERON-TB ${ }^{\circledR}$ Gold In-Tube, Fa. Cellestis, Australien; QFT) bzw. der Bestimmung der Zahl IFN- $\gamma$-sezernierender T-Lymphozyten aus isolierten peripheren mononukleären Zellen (PBMC; T-SPOT.TB ${ }^{\circledR}$, Fa. Oxford-Immunotec, Großbritannien). Diese beiden Tests werden täglich und flächendeckend von mikrobiologischen Instituten sowie kommerziellen Laboren angeboten.

Auch bei CED-Patienten geht der Tuberkulose-Hauttest mit einer hohen Rate an falsch negativen Ergebnissen einher [383]. Ein hoher Anteil von immunsuppressiv behandelten CED-Patienten reagierten anerg auf intradermal applizierte Testantigene (PPD, Candida, Tetanus und/oder Mumps) (83 vs. 43\%, $\mathrm{p}<0,002$ ) [412]. Schoepfer et al. verglichen kürzlich bei 168 CED-Patienten den Tuberkulose-Hauttest mit einem IGRA-Testverfahren und konnten ebenfalls zeigen, dass der TuberkuloseHauttest bei immunsupprimierten Patienten mit einer hohen Rate an falsch negativen Befunden einhergeht. So hatten nur zwei der 14 Patienten mit einem positiven IGRA-Test auch einen positiven Tuberkulose-Hauttest. Darüber hinaus wurde eine hohe Rate an falsch positiven Ergebnissen gefunden. Unter den Patienten, die zuvor BCG geimpft wurden, hatten lediglich $10 \%$ einen positiven IGRA-Test, jedoch $23 \%$ einen positiven Tuberkulose-Hauttest. Diese Daten belegen, dass es auch bei CED-Patienten eine hohe Rate an falsch negativen und auch falsch positiven Tuberkulose-Hauttest-Ergebnissen gibt. Weitere Vorteile des IGRA sind, dass Applikationsfehler und Hautalterationen wie beim THT beim IGRA vermieden werden und der Proband nur einmal für die Durchführung des Tests in der Praxis erscheinen muss. Darüber hinaus tritt kein BoosterEffekt auf, d.h. der Test kann beliebig wiederholt werden, und die Testauswertung ist objektiv und unabhängig vom Ableser. Allerdings bedarf der IGRA eines qualitätsgesicherten Labors und die Vorgaben über Blutentnahme und Transport erfordern eine erprobte Logistik. Ein IGRA kann ebenfalls nicht interpretierbar sein (sogenannte „indeterminate results“) oder falsch negative Ergebnisse aufweisen. Bei einem nicht interpretierbaren IGRA sollte daher zunächst der Test wiederholt werden, um eine ordnungsgemäße Ausführung sicherzustellen, und/ oder der jeweils andere IGRA durchgeführt werden. Bleibt das Testergebnis bei Wiederholung erneut unsicher, kann das Vorliegen einer MTB-Infektion auf diese Weise nicht beurteilt werden kann $[384,406]$. In diesem Fall soll die Entscheidung über eine chemopräventive Therapie anhand der Anamnese und der Röntgenuntersuchungen getroffen werden. Ein Tuberkulose-Hauttest kann zusätzlich durchgeführt werden.

\section{Empfehlung 6.6}

Bei einer latenten Tuberkulose soll eine Therapie nach RKI-Empfehlungen durchgeführt werden. Eine anti-TNF- $\alpha$-Therapie soll frühestens 4 Wochen nach Beginn der latenten Tuberkulose-Therapie durchgeführt werden. Eine strenge Indikationsstellung für die anti-TNF- $\alpha-A K$ soll eingehalten werden.

[Evidenzgrad: D, Konsensusstärke: starker Konsens, Empfehlungsstärke: $\uparrow \uparrow$ ]

\section{Erläuterung}

Die hier genannten Empfehlungen zur Therapie einer LTBI richten sich nach den Empfehlungen des Deutschen Zentralkomitees zur Bekämpfung der Tuberkulose [413]. Neben der durch einen IGRA oder TBC-Hauttest gestellten Indikation zur Chemoprävention vor immunsuppressiver Therapie werden unabhängig davon auch radiologische Zeichen einer durchgemachten, aber unbehandelten Tuberkulose ohne Anhalt für Aktivität (kalzifizierte Knötchen, Spitzenfibrose, Pleuraschwielen) als Indikation zur Chemoprävention betrachtet. Als immunsuppressive Therapie wird hierbei eine Therapie mit TNF- $\alpha$-Antikörpern, mehr als $15 \mathrm{mg}$ Prednisolonäquivalent/Tag für mehr als einem Monat oder eine sonstige iatrogene Immunsuppression betrachtet $[406,413]$. Als chemopräventive Therapie der Wahl, auch für Patienten vor Beginn einer immunsuppressiven Therapie, inklusive anti-TNF- $\alpha$-Antikörper, wird die Gabe von Isoniazid (INH) mit einer Tagesdosis von $5 \mathrm{mg} / \mathrm{kg}$ Körpergewicht (maximal $300 \mathrm{mg}$ ) für 9 Monate empfohlen. Folgt man dieser Empfehlung konsequent, wäre auch vor einer Steroidstoßtherapie oder bei Einleitung einer Behandlung mit Azathioprin bei positivem Tuberkulosescreening eine INH-Prophylaxe durchzuführen. Bei Isoniazid-Unverträglichkeit kann alternativ eine Chemoprävention mit Rifampicin über 4 Monate erfolgen, wobei dieses Regime für Patienten unter anti-TNF- $\alpha$ Therapie nicht ausreichend evaluiert ist. Ist der Patient aus einem Herkunftsland mit bekannt erhöhter INH-Resistenz (z.B. Russland) immigriert, so wird eine chemopräventive Therapie mit Rifampicin und Pyrazinamid über 3 Monate empfohlen (Einzelheiten s. [413]). Zum jetzigen Zeitpunkt gibt es keinen Anhalt, dass die INH-Monotherapie eine spätere INH-Resistenz verursacht, sollte es zu einer aktiven Tuberkuloseerkrankung kommen [414].

Der beste Zeitpunkt für den Beginn einer Anti-TNF- $\alpha$-Therapie bei Patienten, die sich einer INH-Chemoprävention unterziehen, ist nicht bekannt. Idealerweise sollte die Therapie mit TNF- $\alpha$-Antikörpern erst nach Abschluss der INH-Chemoprävention begonnen werden. Sofern die klinische Aktivität dieses nicht zulässt, ist frühestens 1-2 Monate nach Einleitung der Chemoprävention mit der Therapie zu beginnen. Bei guter Adhärenz ist mit einer Effektivität der INH-Therapie von etwa $80 \%$ zu rechnen, sodass die Patienten vor Beginn der antiTNF- $\alpha$-Therapie über das Risiko einer Tuberkulose-Erkrankung trotz Chemoprophylaxe aufgeklärt werden müssen und während und bis zu einem Jahr nach Beendigung der anti-TNF- $\alpha$ - 
Therapie auf Zeichen einer Tuberkulose geachtet werden muss [401, 406, 415].

\section{Empfehlung 6.7}

Bei einer manifesten TB soll eine Therapie nach RKI-Empfehlungen durchgeführt werden. Eine anti-TNF- $\alpha$-Therapie soll - bei stets strenger Indikationsstellung - idealerweise erst nach Beendigung der Tuberkulose-Therapie durchgeführt werden.

[Evidenzgrad: D, Konsensusstärke: starker Konsens, Empfehlungsstärke: $\uparrow \uparrow$ ]

\section{Erläuterung}

Bezüglich der Behandlung einer aktiven Tuberkulose wird auf die Empfehlungen des Deutschen Zentralkomitees zur Bekämpfung der Tuberkulose und des Robert Koch-Instituts verwiesen [416, 417]. Ob unter laufender tuberkulostatischer Therapie eine anti-TNF- $\alpha$-Therapie eingeleitet werden kann, ist nicht bekannt. Eine anti-TNF- $\alpha$-Therapie sollte idealerweise erst nach Beendigung der Tuberkulose-Therapie durchgeführt werden. Eine kritische Diskussion mit dem Patienten und strenge Indikationsstellung für die anti-TNF- $\alpha$-Therapie wird insbesondere in dieser Situation empfohlen.

\section{Empfehlung 6.8}

Bei positivem Toxinnachweis oder endoskopischen oder histologischen Zeichen einer Clostridium-difficile-Erkrankung soll eine antibiotische Therapie mit Metronidazol an erster Stelle durchgeführt werden. Bei schwerer, insbesondere fulminanter Verlaufsform oder fehlendem Ansprechen soll mit Vancomycin p. o. behandelt werden. Lokale Resistenzen sind zu beachten.

[Evidenzgrad: B, Konsensusstärke: Konsens, Empfehlungsstärke: $\uparrow \uparrow]$

\section{Erläuterung}

Eine CED ist ein unabhängiger Risikofaktor für eine Clostridium-difficile-Infektion (Morbus Crohn OR2,1, 95\% CI1,3-3,4; Colitis ulcerosa OR 4,0, 95\% CI2,4-6,6). Zwischen 1993 und 2003 konnte eine Zunahme der Inzidenz bei CED-Patienten beobachtet werden (CU: $17 / 1000$ vs. 36/1000, MC: 0,9/1000 vs. 1,3/1000 Entlassungen) [66]. CED-Patienten mit einer C.difficile-Infektion weisen, im Gegensatz zu CED-Patienten ohne C.-difficile-Erkrankung, eine längere Krankenhausverweildauer und eine vierfach höhere Mortalität auf [418]. Erfahrungen bei CED-Patienten sowie aus dem Bereich der Transplantationsmedizin zeigen, dass eine immunsuppressive Therapie mit einer höheren Inzidenz (OR 2,5 für AZA/6-MP oder MTX) und höherem Schweregrad der Erkrankung einhergeht $[65,419]$. Nachdem kleinere Studien keinen Zusammenhang zwischen einer Kortikosteroidtherapie und einer C.-difficile-Infektion zeigen konnten [54, 420], wurde bei 10662 CEDPatienten im Vergleich zu AZA/6-MP und MTX eine Verdreifachung des Risikos für eine C.-difficile-Infektion bei Glukokortikoidtherapie (RR 3,4; 95\% CI1,9-6,1) beobachtet [357]. Ob die Therapie mit einem anti-TNF- $\alpha$-Antikörper mit einem erhöhten Risiko einhergeht, ist unklar. Weitere Risikofaktoren sind eine (stattgehabte) Antibiotikatherapie oder die Ernährung über eine nasogastrale Sonde/PEG [421]. CED-Patienten mit C.-difficile-Erkrankung weisen bei einer Protonenpumpeninhibitor-Therapie ein knapp vierfach erhöhtes Rezidivrisiko für eine Clostridium-difficile-Kolitis auf [422]. Die Diagnose einer
C.-difficile-Infektion gilt als gesichert bei entsprechender klinischer Symptomatik und Toxin A- und/oder -B-Nachweis oder positiver C.-difficile-Kultur mit Toxinnachweis im Überstand oder dem endoskopischen Nachweis einer pseudomembranösen Colitis, wobei in einer Multicenterstudie nur bei $5 \%$ der CED-Patienten mit einer C.-difficile-Infektion histologisch Pseudomembranen nachweisbar waren [423]. (Zur Diagnosestellung siehe auch Kapitel 2.)

Der erste Schritt in der Behandlung einer C.-difficile-Infektion besteht in einer Kontaktisolation, Kittel- und Handschuhpflege sowie Händereinigung mit Seife und sporiziden Desinfektionsmitteln [424]. Für die medikamentöse Therapie einer C.-difficile-Infektion gibt es unterschiedliche Empfehlungen, die jedoch nahezu alle eine antibiotische Therapie mit Metronidazol oder Vancomycin einschließen und sich nur in der Dosierung und Dauer der Medikation sowie Art des primär zu verwendenden Antibiotikums unterscheiden [425]. Kontrollierte Studien zur Behandlung einer C.-difficile-Infektion bei CED-Patienten fehlen. Die oben formulierten Empfehlungen stützen sich auf aktuelle Empfehlungen der European Society of Clinical Microbiology and Infectious Diseases (ESCMID) zur Behandlung einer C.-difficile-Infektion [426]. Diese empfehlen schweregradabhängig eine antibiotische Therapie mit Metronidazol oder Vancomycin.

Sofern der klinische Verlauf es zulässt, sollte bei jedem Patienten mit einer C.-difficile-Infektion zunächst eine laufende antibiotische Therapie beendet werden. Für Patienten mit den Zeichen einer leichten bis moderaten C.-difficile-Erkrankung wird eine Therapie mit Metronidazol 400-500 mg p.o. alle 8 Stunden über 10 Tage empfohlen. Sollte es nach 3-5 Tagen zu keiner Besserung kommen, muss von einem Versagen der Therapie ausgegangen werden und ein Wechsel z.B. von Metronidazol auf Vancomycin p.o. oder intravenöse Therapie mit Metronidazol in Betracht gezogen werden. Für Patienten mit einer schweren C.-difficile-Erkrankung (Fieber, Schüttelfrost, hämodynamische Instabilität, Peritonitis, Ileus, Leukozytose $>15 \times 10^{9} / \mathrm{L}$, Linksverschiebung, Laktaterhöhung, toxisches Megakolon) wird eine initiale antibiotische Therapie mit Vancomycin $125 \mathrm{mg} /$ alle 6 Stunden per os für 10 Tage empfohlen. Eine höhere Vancomycin-Dosierung ergibt keinen zusätzlichen Nutzen [427]. Für Patienten, bei denen eine orale antibiotische Therapie nicht möglich ist, wird für leichte Fälle eine intravenöse Therapie mit Metronidazol $500 \mathrm{mg} /$ alle 8 Stunden über 10 Tage und in schweren Fällen eine zusätzliche Vancomycinapplikation (500 mg in $100 \mathrm{ml}$ Kochsalzlösung alle $4-12 \mathrm{~h}$ ) in das Kolon und/oder eine Vancomycingabe (500 mg alle 6 Stunden) über die Nasensonde empfohlen. Eine gleichzeitig mit der C.-difficile-Erkrankung durchgeführte immunsuppressive Therapie sollte soweit wie möglich pausiert oder beendet werden, da immunsuppressiv behandelte CED-Patienten eine höhere Morbidität (u.a. Kolektomie, toxisches Megakolon, Darmperforation) und Mortalität aufwiesen als die Vergleichsgruppe ohne Immunsuppression [422].

Die klinische Beurteilung des Ansprechens auf die antibiotische Therapie ist bei CED-Patienten mit C.-difficile-Infektion, die sich nicht in Remission befinden, bei fortbestehender Diarrhö schwierig. Eine erneute mikrobiologische Stuhldiagnostik als Erfolgskontrolle einer antibiotischen Therapie ist nicht hilfreich, da sowohl C. difficile als auch Toxin A und B noch Wochen nach einer erfolgreichen Behandlung im Stuhl nachgewiesen werden können [428]. Wird jedoch das Rezidiv einer Infektion vermutet, sollte eine erneute Stuhldiagnostik durch- 
geführt werden. Resistenzen gegen Metronidazol wurden beschrieben und müssen, auch wenn sie selten auftreten, bei Therapieversagern beachtet werden [429]. Andere Medikamente zur Behandlung einer C.-difficile-Erkrankung, wie z.B. Rifaximin, Fusidinsäure oder Nitazoxanide, können zum jetzigen Zeitpunkt nicht empfohlen werden. Eine kürzlich durchgeführte Cochrane-Analyse konnte ebenfalls keinen Vorteil für eine, zusätzlich zur Antibiotikatherapie durchgeführte, Gabe von Probiotika nachweisen [430]. Bei einer weiteren Verschlechterung trotz antibiotischer Therapie mit Ileus oder toxischem Megakolon sollte die Option einer (subtotalen) Kolektomie mit Anlage eines Ileostomas in Betracht gezogen werden. Das Rezidiv einer C.-difficile-Erkrankung soll wie eine primäre Episode behandelt werden, weitere Rezidive direkt mit Vancomycin $125 \mathrm{mg}$ /alle 6 Stunden für 10 Tage [428, 431].

Ob asymptomatische CED-Patienten, mit oder ohne immunsuppressiver Therapie, und dem Nachweis von C.-difficile-Toxin im Stuhl antibiotisch behandelt werden müssen, ist nicht bekannt.

\section{Empfehlung 6.9}

Insbesondere vor Einleitung einer immunsuppressiven Therapie sollte eine Beratung zum Gesundheitsverhalten (Ernährung, Reisen) durchgeführt werden.

[Evidenzgrad: D, Konsensusstärke: Konsens, Empfehlungsstärke: $\uparrow$ ]

\section{Erläuterung}

In Analogie zu Empfehlungen für Transplantationspatienten [432] und der Tatsache, dass ein relevanter Anteil von „CEDRezidiven“ infektiöser Genese sind [54, 420], sollte vor Einleitung einer immunsuppressiven Therapie eine Beratung zum Gesundheitsverhalten durchgeführt werden. Aufgrund einer erhöhten Wahrscheinlichkeit infektiöser Diarrhöen (u. a. Escherichia coli, Salmonella spp, Campylobacter spp., Entamoeba histolytica und Listeria monocytogenes) bei einer immunsuppressiven Therapie wird die Einhaltung einer speziellen Nahrungsmittelhygiene empfohlen. Hierzu zählt u.a. das Meiden von Rohmilch, Rohmilchkäse, rohen Eiern, Tartar, rohem Fisch und Meeresfrüchten. Dies trifft v.a. in Phasen einer intensiven Immunsuppression und verstärkt auf Reisen im Ausland zu. Darüber hinaus muss auf ein erhöhtes Risiko einer Tuberkulose-Neuerkrankung bei Reisen in Tuberkulose-Hochendemie-Regionen v.a. in Entwicklungsländer hingewiesen werden.

\section{Empfehlung 6.10}

Bei Erstdiagnose, spätestens aber vor Einleitung einer immunsuppressiven Therapie, soll der Impfstatus überprüft und ggf. aktualisiert werden. Nicht-Lebendimpfungen unter immunsuppressiver Therapie gelten als sicher, während Lebendimpfungen kontraindiziert sind. Vor diesem Hintergrund soll in Analogie zu den Empfehlungen des RKI zu Impfungen bei Immunsuppression gehandelt werden.

[Evidenzgrad: D, Konsensusstärke: starker Konsens, Empfehlungsstärke: $\uparrow \uparrow$ ]

\section{Erläuterung}

Obwohl CED-Patienten mit einer immunsuppressiven Therapie ein erhöhtes Infektionsrisiko besitzen, wird nur ein geringer Teil der Patienten regelmäßig zum Schutz vor Infektionen geimpft. So waren in einer Studie mit 146 immunsuppressiv behandelten CED-Patienten nur 9\% gegen Pneumokokken geimpft, 28\% erhielten eine jährliche Influenza-Schutzimpfung und weniger als die Hälfte der Patienten hatte einen ausreichenden Tetanus-Schutz [433]. In einer anderen Untersuchung wiesen nur $12 \%$ der Patienten einen ausreichenden Schutz gegen Hepatitis B auf [434]. Als Ursache für die niedrige Impfquote wurde in 50\% der Fälle Unkenntnis und in 18\% Angst vor Nebenwirkungen angegeben [433]. Zwar ist in Einzelfällen der Impferfolg - der Schutz vor der jeweiligen Erkrankung - in Abhängigkeit von dem Ausmaß einer immunsuppressiven Therapie nicht immer vorhersehbar, generell werden Impfungen jedoch empfohlen $[435,436]$. Eine Übersicht über die Möglichkeiten einer Überprüfung des Impferfolgs gibt das Epidemiologische Bulletin November 2005, Hinweise zu Impfungen für Patienten mit Immundefizienz der STIKO am RKI [437].

Bei Erstdiagnose bzw. Erstvorstellung eines Patienten mit CED sollte somit der aktuelle Impfstatus kontrolliert und fehlende Impfungen nachgeholt werden. Dabei muss beachtet werden, dass die Gabe von Lebendimpfstoffen (Mumps, Masern, Röteln, Varizellen, Gelbfieber, BCG, Typhus oral und Polio oral) bei immunsupprimierten Patienten kontraindiziert ist.

Keine Kontraindikation für Lebendimpfstoffe stellen dar:

- topische Steroidtherapie/lokale Steroid-Injektionen (z.B. intraartikulär),

- physiologische Kortisonerhaltungsdosis,

- niedrige systemische Kortisondosen (<20 mg/d Prednison; für Patienten $<10 \mathrm{~kg}$ Körpergewicht: $<2 \mathrm{mg} / \mathrm{kg}$ Körpergewicht/ Tag Prednison).

Bei hoher systemischer Glukokortikoideinnahme $(\geq 20 \mathrm{mg} / \mathrm{d}$ Prednisolon bzw. $\geq 2 \mathrm{mg} / \mathrm{kg} / \mathrm{d}$ Prednisolon bei $\mathrm{KG}<10 \mathrm{~kg}$ ) gelten folgende Hinweise:

- Behandlungsdauer < 14 Tage: Impfung mit Lebendimpfstoffen unmittelbar nach Beendigung der Therapie möglich.

- Behandlungsdauer > 14 Tage: Impfung mit Lebendimpfstoffen 1 Monat (Mindestabstand) nach Beendigung der Therapie [437].

Weiterhin gelten eine laufende Therapie mit AZA/6-MP, MTX, Calcineurininhibitor oder anti-TNF- $\alpha$-Antikörpern sowie der Zeitraum von 3 Monaten nach Beendigung der jeweiligen Therapie als Kontraindikation für eine Impfung mit einem Lebendimpfstoff.

Aufgrund dieser Einschränkungen und der Gefahr eines verminderten Impferfolgs unter Immunsuppression sollten ausstehende Impfungen vor Beginn einer langfristigen Immunsuppression durchgeführt werden. Eine Varizellenimpfung sollte mindestens 3 Wochen, eine Mumps-Masern-Röteln-Impfung mindestens 6 Wochen vor Beginn einer immunsuppressiven Therapie abgeschlossen sein und frühestens 3 Monate nach Ende einer immunsuppressiven Therapie begonnen werden [436]. Generell wird für alle CED-Patienten eine Hepatitis-B-Impfung empfohlen. Es gibt keinen Anhalt, dass Impfungen einen CED-Schub auslösen bzw. die CED-Symptomatik beeinflussen [435, 436]. Eine Mumps-Masern-Röteln-Impfung bei Angehörigen von Patienten mit einer immunsuppressiven Medikation ist bedenkenlos möglich. Eine Gefahr einer Virusverbreitung nach der Impfung besteht nicht [438]. Ebenfalls ist eine Varizellen-Impfung bei Angehörigen von Patienten mit einer immunsuppressiven Medikation bedenkenlos möglich. Bei Ausbildung eines Impfausschlags sollten jedoch Varizellen-seronegative immunsupprimierte Patienten den Kontakt mit der Person meiden [439]. 


\section{Empfehlung 6.11}

Eine Influenzaimpfung sowie eine Impfung gegen die pandemische Grippe soll insbesondere bei Patienten mit einer medikamentösen Immunsuppression jährlich durchgeführt werden.

[Evidenzgrad: B, Konsensusstärke: starker Konsens, Empfehlungsstärke: $\uparrow \uparrow]$

\section{Erläuterung}

Für Patienten mit einer immunsuppressiven Therapie wird von der STIKO eine jährliche Influenza-Impfung im Herbst sowie eine Impfung gegen die Neue Influenza (H1N1) empfohlen [437, 440]. So reduziert die Impfung gegen Influenza die Morbidität und Mortalität von Patienten, die älter als 65 Jahre sind. Aber auch jüngere Patienten profitieren von einer Influenza-Impfung, indem die „Grippeerkrankungen“ und Krankheitstage deutlich reduziert werden [441]. Zwei Studien haben die Impfantwort nach einer Influenza-Impfung bei rund 200 Kindern bzw. Jugendlichen mit einer CED untersucht [442, 443]. Im Vergleich zu einer Kontrollgruppe fand sich weder für Patienten ohne Immunsuppressiva noch für Patienten mit einer immunsuppressiven Therapie ein Unterschied in der Impfantwort. Nur in der Sub-Gruppe der mit anti-TNF- $\alpha$-behandelten CED-Patienten zeigte sich eine verminderte Impfantwort, v.a. auf den Influenza-B-Subtyp.

\section{Empfehlung 6.12}

Eine Hepatitis-B-Impfung sollte bei allen seronegativen CED-Patienten durchgeführt werden. Eine Titerkontrolle soll bei Patienten mit immunsuppressiver Medikation durchgeführt werden.

[Evidenzgrad: D, Konsensusstärke: starker Konsens, Empfehlungsstärke: $\uparrow \uparrow$ ]

\section{Erläuterung}

Grundsätzlich wird eine HBV-Impfung für Patienten mit einer immunsuppressiven Medikation durch die STIKO nicht explizit empfohlen. Es gibt keinen Hinweis, dass das Risiko einer HBV-Infektion im Vergleich zur Normalbevölkerung bei CEDPatienten erhöht ist [434]. Die STIKO verweist aber auf die Impfnotwendigkeit bei „Patienten mit ... häufiger Übertragung von Blut(bestandteilen) und vor ausgedehntem chirurgischen Eingriff...“ [444]. Aufgrund der schwerwiegenden Folgen einer Hepatitis-B-Reaktivierung unter einer immunsuppressiven Therapie und teils ausgedehnter chirurgischer Eingriffe und Bluttransfusionen im Rahmen der CED-Erkrankung sollten daher alle seronegativen CED-Patienten eine HBV-Impfung (z.B. ENGERIX $^{\circledR}$ nach Herstellerangaben) erhalten. Eine Kontraindikation für eine HBV-Impfung unter immunsuppressiver Therapie besteht nicht, jedoch wird eine serologische Impferfolgskontrolle bei Patienten mit Immunsuppression empfohlen. So wiesen mehr als die Hälfte der immunsuppressiv behandelten CED-Patienten nach Hepatitis-B-Impfung keinen ausreichenden anti-HBs-Titer auf [445]. Bei anti-HBs-Werten kleiner als 100 IE/l nach erfolgter Impfung ist eine sofortige Wiederimpfung mit erneuter Kontrolle durchzuführen; bei erneutem Nichtansprechen wird eine Wiederimpfung mit in der Regel max. 3 Dosen empfohlen. Alternativ zu diesem Vorgehen konnte eine hohe Erfolgsrate (95\%) durch Verabreichung einer doppelten Dosis eines kombinierten Hepatitis-A- und -B-Wirkstoffs (TWINRIX ${ }^{\circledR}$, Monat 0, 1 und 6) bei „Non-Respondern“ erreicht werden [446].

\section{Empfehlung 6.13}

Eine VZV-Impfung soll bei allen CED-Patienten mit negativer VZVAnamnese (Windpocken/Herpes zoster) bzw. negativer VZV-Serologie durchgeführt werden. Eine Impfung bei vorliegender Immunsuppression ist kontraindiziert.

[Evidenzgrad: D, Konsensusstärke: starker Konsens, Empfehlungsstärke: $\uparrow \uparrow$ ]

\section{Erläuterung}

Im Juli 2004 hat die STIKO am RKI die Varizellenimpfung für alle Kinder in Ihre Empfehlungen aufgenommen [447]. Die Durchseuchungsrate in der Altersgruppe der 10- bis 11-Jährigen liegt bei $94 \%$. Bei Jugendlichen und Erwachsenen bis zu 40 Jahren bestehen noch Immunitätslücken von ca. 3-4\% [447]. Neben der generellen Impfempfehlung empfiehlt die STIKO für alle seronegativen Patienten vor geplanter immunsuppressiver Therapie eine Varizellen-Impfung aufgrund des schweren Verlaufs einer Varizelleninfektion bei Erwachsenen mit einer Mortalität von 20:100000 (Mortalität von 2:100000 im Kindesalter). Für die Empfehlung spricht auch die Tatsache, dass bei 30\% der immunsuppremierten Patienten die Erkrankung mit einem disseminierten Verlauf einhergeht [439]. So wurden mehrere schwere Fälle von Varizellen-Infektionen, sowohl Windpocken- als auch Herpes-Zoster-Erkrankungen, bei CED-Patienten unter immunsuppressiver Therapie mit u.a. AZA/6-MP und Infliximab beschrieben [448-450]. Gupta et al. beschrieben bei CED-Patienten im Vergleich zur Allgemeinbevölkerung ein 1,5-fach erhöhtes Risiko von Herpes-zoster-Infektionen für Patienten die mit Kortikosteroiden (95\% CI1,1 2,2 ) und ein dreifach erhöhtes Risiko für Patienten, die mit AZA/6-MP (95\% CI 1,7-5,6) behandelt wurden [350]. Aufgrund dessen sollten bei jedem Patienten vor Einleitung einer immunsuppressiven Therapie eine Varizellenanamnese und eine Beurteilung des Impfstatus erfolgen. Bei Unklarheiten können serologische Kontrollen (IgG) hilfreich sein. Seronegative Patienten oder Patienten ohne Varizellenanamnese (ca. 10-15\% der Gesamtpopulation in Deutschland) sollen vor Beginn der immunsuppressiven Therapie eine Varizellenimpfung (2 Dosen nach Herstellerangaben) erhalten. Da es sich bei den Impfstoffen um einen attenuierten Lebendimpfstoff handelt, ist die prophylaktische Gabe während einer immunsuppressiven Therapie kontraindiziert. Die Varizellen-Impfung sollte mindestens 3 Wochen vor Beginn einer immunsuppressiven Therapie abgeschlossen sein und frühestens 3 Monate nach Ende einer immunsuppressiven Therapie begonnen werden [436, 439]. Bei immundefizienten Patienten mit unbekannter oder fehlender Varizellen-Immunität und Kontakt zu einer Person mit Varizella-Zoster-Infektion wird, in Übereinstimmung mit den Empfehlungen des RKI, empfohlen, innerhalb von 96 Stunden nach Exposition (1 Stunde oder länger mit infektiöser Person in einem Raum, face-to-face-Kontakt, Haushaltskontakt) eine postexpositionelle passive Immunisierung mit Varizella-ZosterImmunglobulin (VZIG) durchzuführen. Sie kann den Ausbruch einer Erkrankung verhindern oder deutlich abschwächen. Für Applikation und Dosierung von VZIG sind die Herstellerangaben zu beachten. 


\section{Empfehlung 6.14}

Eine HPV-Impfung soll bei Mädchen und jungen Frauen insbesondere mit immunsuppressiver Therapie nach RKI-Empfehlungen durchgeführt werden.

[Evidenzgrad: B, Konsensusstärke: Konsens, Empfehlungsstärke: $\uparrow \uparrow]$

\section{Erläuterung}

Seit 2006 steht ein Impfstoff gegen humane Papillomviren (HPV) der Typen 6, 11, 16 und 18 und seit 2007 ein Impfstoff gegen HPV der Typen 16 und 18 zur Verfügung. Die STIKO hat eine Empfehlung zur Impfung gegen HPV für alle 12-17 Jahre alten Mädchen ausgesprochen und diese zuletzt 2009 bestätigt [451]. Als Impfziel ist die Reduktion der Krankheitslast durch das Zervixkarzinom definiert. Nachdem bei CED-Patienten, insbesondere bei Patientinnen mit immunsuppressiver Therapie, ein erhöhtes Risiko für die Entwicklung zervikaler Dysplasien beschrieben wurde [452, 453], sollte die HPV-Impfung in dieser Patientengruppe durchgeführt werden. Eine Kontraindikation für Patienten mit laufender immunsuppressiver Therapie besteht nicht.

\section{Empfehlung 6.15}

Insbesondere Patienten mit immunsuppressiver Therapie sollen gegen Pneumokokken geimpft werden.

[Evidenzgrad: D, Konsensusstärke: Konsens, Empfehlungsstärke: $\uparrow \uparrow$ ]

\section{Erläuterung}

In den aktuellen Empfehlungen der STIKO von 2009 wird vor Beginn einer immunsuppressiven Therapie die Durchführung einer Pneumokokken-Schutzimpfung mit einem PolysaccharidImpfstoff (PNEUMOVAX ${ }^{\circledR}$ ) empfohlen [444]. So sind bei Patienten mit immunsuppressiver Therapie eine ggf. auch mehrere Wiederholungsimpfungen mit Polysaccharid-Impfstoff im Abstand von 5 (Erwachsene) bzw. mindestens 3 Jahren (Kinder unter 10 Jahren) in Erwägung zu ziehen (Risiko-Nutzen-Abwägung beachten). Untersuchungen zeigen, dass in diesem Kollektiv weniger funktionelle Antikörper oder sehr schnell abfallende Antikörper-Titer vorliegen, sodass hier eine oder mehrere Wiederholungsimpfungen mit einem PolysaccharidImpfstoff die Antikörper-Antwort verlängern und möglicherweise damit die Schutzwirkung verbessern können [437, 444]. Untersuchungen der Immunantwort bzw. des Impferfolgs nach Pneumokokken-Schutzimpfung bei Patienten mit immunsuppressiver Therapie erfolgten $u$.a. bei Patienten mit einer rheumatoiden Arthritis (RA) [454-456] und bei Patienten mit CED [457]. Eine immunsuppressive Kombinationstherapie mit einem anti-TNF- $\alpha$-Antikörper beeinträchtigt ebenfalls die Immunantwort nach einer Pneumokokken-Schutzimpfung. So weisen nur 45\% der CED-Patienten mit einer immunsuppressiven Kombinationstherapie im Gegensatz zu 80\% der CED-Patienten ohne Immunsuppression und 85\% der gesunden Personen in der Kontrollgruppe einen adäquaten AntikörperTiteranstieg nach Pneumokokken-Impfung (PPS-23) auf [457]. Für eine anti-TNF- $\alpha$-Monotherapie bei CED-Patienten liegen keine Daten vor. Beobachtungen bei Patienten mit rheumatoider Arthritis deuten jedoch darauf hin, dass eine Monotherapie den Impferfolg nicht beeinflusst $[455,458]$.
Insgesamt wird somit empfohlen, vor Beginn einer immunsuppressiven Therapie die Pneumokokken-Schutzimpfung durchzuführen. Sollte dies nicht möglich sein, ist die Durchführung einer Pneumokokken-Schutzimpfung auch unter fortgeführter immunsuppressiver Therapie zu erwägen. Es besteht keine Kontraindikation für eine Impfung unter Immunsuppression. Eine Wiederholungsimpfung mit Polysaccharid-Impfstoff im Abstand von 5 (Erwachsene) bzw. mindestens 3 Jahren (Kinder unter 10 Jahren) wird bei fortbestehender Immunsuppression empfohlen.

\section{Empfehlung 6.16}

Patienten, die eine dreifach immunsuppressive Therapie erhalten, sollen eine PJP-Prophylaxe mit Trimethoprim und Sulfamethoxazol erhalten.

[Evidenzgrad: D, Konsensusstärke: Konsens, Empfehlungsstärke: $\uparrow \uparrow]$

\section{Erläuterung}

Es gibt keine systematisch erhobenen Daten über das Risiko von CED-Patienten, mit (mehrfach) immunsuppressiver Therapie an einer Pneumocystis-jiroveci-Pneumonie (PJP) zu erkranken. Bisherige Empfehlungen über eine PJP-Prophylaxe basieren auf Expertenmeinungen und auf Erfahrungen aus anderen Fachgebieten $[459,460]$. Eine kürzlich durchgeführte Metaanalyse konnte zeigen, dass eine chemopräventive Therapie mit Sulfamethoxazol/Trimethoprim bei Patienten mit hämatologischen Erkrankungen oder nach Transplantation eine Abnahme der PJP um 91\% bedingt [461]. Sowohl unter einer Therapie mit AZA/6-MP und MTX als auch unter einer Therapie mit Infliximab wurden Fälle einer PJP beschrieben. Das höchste PJP-Risiko ist mit einer Ciclosporin-Therapie assoziiert, wenngleich angemerkt werden muss, dass nahezu alle berichteten PJP-Fälle bei CED-Patienten unter einer Kombinationstherapie aus mehreren Immunsuppressiva aufgetreten sind [460]. Für eine positive Empfehlung bei einer zweifach immunsuppressiven Therapie, die einen Calcineurininhibitor (CsA oder Tacrolimus) oder einen anti-TNF- $\alpha$ Antikörper mit einem anderen Immunsuppressivum (z.B. Glukokortikoide) kombiniert, konnte kein Konsensus erreicht werden [462]. Zur Durchführung einer PJP-Prophylaxe wird Sulfamethoxazol/ Trimethoprim empfohlen. Dabei geht die $3 \times$ wöchentliche Einnahme von Sulfamethoxazol/Trimethoprim $(800 / 160 \mathrm{mg}) \mathrm{mit}$ weniger Nebenwirkungen einher als die tägliche Einnahme von Sulfamethoxazol/Trimethoprim (400/80 mg) [463]. Bei einer Sulfonamid-Allergie wird die Hinzuziehung eines Infektiologen angeraten.

\section{Empfehlung 6.17}

Bei Neugeborenen von Müttern mit Colitis ulcerosa treten, unabhängig von einer immunsuppressiven Behandlung der Mutter, gehäuft bakterielle perinatale Infektionen auf. Die Neugeborenen sollen daher in der Perinatalperiode entsprechend überwacht werden.

[Evidenzgrad: D, Konsensusstärke: starker Konsens, Empfehlungsstärke: $\uparrow \uparrow$ ]

\section{Erläuterung}

Bengtson et al. konnten kürzlich zeigen, dass das Risiko einer perinatalen bakteriellen Infektion bei Neugeborenen von Müttern mit Colitis ulcerosa (aber nicht mit Morbus Crohn), die 
keine immunsuppressive Therapie erhalten haben, ca. 6-fach erhöht ist [464]. Auch wenn es sich bei der Studie nur um eine kleine Studie handelt und eine Bestätigung dieser Beobachtung durch größere Studien fehlt, ist die engmaschige Überwachung dieser Neugeborenen auf infektiöse Komplikationen anzuraten.

\section{Statement 6.18}

Ein validierter Algorithmus zur Diagnose einer CMV-Erkrankung, insbesondere bei Patienten mit immunsuppressiver Therapie, ist nicht vorhanden.

[Evidenzgrad: D, Konsensusstärke: starker Konsens]

\section{Empfehlung 6.19}

Eine CMV-Erkrankung soll behandelt werden, wenn klinische Symptome bestehen und mindestens einer der folgenden Parameter erfüllt sind:

a) positive CMV-PCR im Blut mit signifikanter Viruslast,

b) positive Immunhistologie bzw.

c) klassische Histologie („Eulenaugenzellen“).

[Evidenzgrad: D, Konsensusstärke: starker Konsens, Empfehlungsstärke: $\uparrow \uparrow$ ]

\section{Erläuterung}

Um Redundanz zu vermeiden, wird bez. der Diagnose einer CMV-Infektion und CMV-Erkrankung auf den Diagnostikteil verwiesen (siehe Kapitel 2, Empfehlung 2.16).

\section{Empfehlung 6.20}

Bei einer CMV-Erkrankung, die entsprechend den oben formulierten Kriterien diagnostiziert wurde, soll eine antivirale Therapie durchgeführt werden.

[Evidenzgrad: B, Konsensusstärke: starker Konsens, Empfehlungsstärke: $\uparrow \uparrow$ ]

\section{Empfehlung 6.21}

Die Akuttherapie soll bis zum Nachweis einer negativen CMV-PCR im Blut, jedoch für mindestens 14 Tage durchgeführt werden. [Evidenzgrad: D, Konsensusstärke: starker Konsens, Empfehlungsstärke: $\uparrow \uparrow$ ]

\section{Erläuterung}

Die Standardsubstanz zur Therapie der CMV-Erkrankung ist derzeit Ganciclovir ( $5 \mathrm{mg} / \mathrm{kg}$ i.v. alle $12 \mathrm{~h}$ ), die bei systemischen CMV-Infektionen Erfolgsraten von 70-80\% erzielt [465, 466]. Die Substanz weist mehrere potenziell schwere Nebenwirkungen auf. So können bei bis zu 40\% der Patienten Nierenfunktionsstörungen und eine Myelosuppression auftreten [467]. Bei Therapieversagen, wie z.B. bei der selten zu beobachtenden Ganciclovir-Resistenz oder Kontraindikationen, kann auf Foscarnet (90 mg/kg i.v. alle $12 \mathrm{~h}$, oder $60 \mathrm{mg} / \mathrm{kg}$ i.v. alle $8 \mathrm{~h}$ ) ausgewichen werden, das in kontrollierten Studien vergleichbare Ansprechraten zeigte. Da bis zu einem Drittel der Patienten auch unter einer Foscarnet-Therapie ebenfalls schwere Nebenwirkungen (z.B. Nierenfunktionsstörungen und neurologische Neben- wirkungen) entwickeln, sollte eine Therapie nur bei klinisch fassbaren CMV-Erkrankungen durchgeführt werden.

Während einer CMV-Therapie sollte ein regelmäßiges CMV-PCRMonitoring im Blut erfolgen, erstmalig 7 Tage nach Therapiebeginn, um ein Therapieansprechen bzw. -versagen bei möglicher Resistenz zu erkennen. Die Akuttherapie soll bis zum Nachweis einer negativen CMV-PCR im Blut, jedoch für mindestens 14 Tage durchgeführt werden. Empfehlungen aus dem Bereich der Onkologie sehen eine Akuttherapie bis 7 Tage nach erstmaliger negativer CMV-PCR im Blut vor [468, 469].

Neuerdings steht als oral applizierbares Derivat von Ganciclovir, Valganciclovir (2×täglich $900 \mathrm{mg}$ per os) zur Verfügung, das in einer randomisierten Studie zur CMV-Retinitis in der Initialtherapie ebenso gute Behandlungsergebnisse ergab wie das parenteral zu verabreichende Ganciclovir [470]. Humar et al. verglichen bei organtransplantierten Patienten mit einer CMV-Erkrankung die orale Valganciclovir-Therapie $(\mathrm{n}=32) \mathrm{mit}$ einer parenteralen Ganciclovir Therapie $(n=32)$. Sowohl die virale Clearance am Tag 21 (50 vs. 46.9\%) als auch der Abfall der Viruslast am Tag 7 und 14 zwischen beiden Gruppen war nicht signifikant unterschiedlich [471]. Weitere Studien im Bereich der Organtransplantation kamen zu dem Ergebnis, dass eine primäre Valganciclovir-Therapie im Vergleich zu einer sequenziellen Therapie aus Ganciclovir-Valganciclovir, gleichwertig erscheint [472, 473]. In beiden Studien wurden jedoch nur Patienten mit asymptomatischer Virämie oder leichtgradiger CMV-Erkrankung unter Ausschluss von schweren CMV-Erkrankungen untersucht, sodass eine Übertragung der Daten auf andere Patientenkollektive schwierig erscheint. Nichtsdestotrotz wurde, abhängig vom klinischen Bild, nach 2-3 Tagen initialer parenteraler Ganciclovir-Therapie eine Fortsetzung der Therapie mit Valganciclovir von mehreren Autoren als Möglichkeit betrachtet [462, 466, 474]. Kontrollierte Daten zur Therapie der CMV-Kolitis liegen jedoch nicht vor.

Einschränkend muss hinzugefügt werden, dass die Zulassung für Valganciclovir (Valcyte ${ }^{\circledR}$ ) auf die Initial- und Erhaltungstherapie der CMV-Retinitis bei Patienten mit erworbenem ImmundefektSyndrom (AIDS) und die Prophylaxe einer CMV-Erkrankung bei CMV-negativen Patienten, die ein Organtransplantat von einem CMV-positiven Spender erhalten haben, beschränkt ist. Anzumerken ist, dass pharmakokinetische Daten von stammzelltransplantierten Patienten mit einer schweren intestinalen Graft-vs.Host-Reaktion auf eine potenziell verminderte Resorption von Valganciclovir hinweisen. Bei diesen Patienten wird ab dem Schweregrad III nach den Common-Toxicity-Kriterien (7-9 Stühle pro Tag) eine intravenöse Therapie mit Ganciclovir empfohlen [475]. Daten zur Pharmakokinetik bei Patienten mit chronisch entzündlichen Darmerkrankungen liegen nicht vor. Bezüglich der Dosierung von Ganciclovir, Foscarnet und Valganciclovir in besonderen klinischen Situationen, wie z. B. bei Patienten mit Nierenfunktionsstörungen oder in der Pädiatrie, wird auf die Fachinformation verwiesen.

\section{Empfehlung 6.22}

Bei nachgewiesener CMV-Erkrankung sollte bei Wiedereinführung bzw. Fortführung oder Intensivierung der immunsuppressiven Therapie eine Rezidivprophylaxe durchgeführt werden. Diese Therapie schließt sich der Akuttherapie an (s. oben) und sollte für 4 8 Wochen durchgeführt werden.

[Evidenzgrad: D, Konsensusstärke: Konsens, Empfehlungsstärke: $\uparrow]$ 


\section{Erläuterung}

Bei Wiedereinführung bzw. Fortführung oder Intensivierung einer immunsuppressiven Therapie wird bei stattgehabter, erfolgreich behandelter CMV-Infektion eine Rezidivprophylaxe angeraten. Diese ist mit Valganciclovir (einmal täglich $900 \mathrm{mg}$ ) über 4-8 Wochen durchzuführen. Daten, die dieses Vorgehen bei CED-Patienten unterstützen, sind nicht vorhanden. Es handelt sich somit um eine Expertenmeinung.

Trotz eines beobachteten Wiederauftretens einer CMV-Erkrankung nach erfolgreicher Therapie bei 15 -35\% organtransplantierter Patienten ist in dieser Gruppe der Nutzen im Vergleich zu den Risiken einer Sekundärprophylaxe nicht eindeutig belegt. Faktoren, die ein erneutes Auftreten begünstigen, beinhalten u.a. eine primäre CMV-Infektion, Erkrankung mehrerer Organe und eine hohe Viruslast [469, 476, 477].

\section{Kapitel 7: Chirurgie}

$\nabla$

\section{Empfehlung 7.1}

Als Standardoperation soll eine restaurative Proktokolektomie durchgeführt werden.

[Evidenzgrad: D, Konsensusstärke: starker Konsens, Empfehlungsstärke: $\uparrow \uparrow]$

\section{Erläuterung}

Die restaurative Proktokolektomie hat sich in den letzten 30 Jahren als Standardoperation zur Behandlung der Colitis ulcerosa etabliert. Die ileoanale Pouchoperation erzielt für den Patienten eine bestmögliche Lebensqualität mit durchschnittlich 5-6 Stuhlgängen pro Tag und dem Erhalt der Kontinenz in über $90 \%$ der Patienten $[478,479]$.

\section{Empfehlung 7.2*}

Die freie oder gedeckte Perforation soll als Notfallindikation operiert werden.

[Evidenzgrad: A, Konsensusstärke: starker Konsens, Empfehlungsstärke: $\uparrow \uparrow$ ]

\section{Erläuterung}

Die freie oder gedeckte Perforation stellt die schwerste Komplikation der Colitis ulcerosa dar. Die klinische Symptomatik wird hierbei durch eine bestehende Immunsuppression und/ oder Antibiotikatherapie häufig moduliert, sodass eine operative Therapie vor Eintreten septischer Komplikationen durchgeführt werden sollte. In der Hälfte der Fälle geht der Perforation jedoch kein Megakolon voraus. Trotz operativer Therapie beträgt die Mortalität derzeit bis zu 20\% [480]. Um die Mortalität dieser schwersten Komplikation zu senken, stellt die rechtzeitige Operation die entscheidende Maßnahme dar [481].

\section{Empfehlung 7.3}

Die therapierefraktäre Blutung soll bei fortgesetzter Transfusionspflichtigkeit im interdisziplinären Kontext dringlich operiert werden.

[Evidenzgrad: D, Konsensusstärke: Konsens, Empfehlungsstärke: $\uparrow \uparrow]$

\section{Erläuterung}

Die Inzidenz der schweren Blutung bei Patienten mit CU beträgt bis zu 4,5\% [482]. Sie ist verantwortlich für 5\% der Notfalleingriffe. Als Operationsindikation wird entweder eine massive initiale Blutung mit Kreislaufinstabilität und Katecholaminpflichtigkeit oder im Verlauf ein Transfusionsbedarf von mehr als 4 Erythrozytenkonzentraten pro 24 Stunden angesehen.

Für Kinder ist eine Transfusionsbedürftigkeit von $45-60 \mathrm{ml}$ Erythrozytenkonzentrat/kg KG in 24 Stunden als akut bedrohlich, bei anhaltender Blutung über 2 - 3 Tage $30 \mathrm{ml} / \mathrm{Kg}$ Körpergewicht anzusehen. Wenn möglich, sollten Kinder mit schwerem akutem Schub einer Colitis ulcerosa in ein Zentrum mit kindergastroenterologischer und chirurgischer Expertise verlegt werden.

\section{Empfehlung $7.4^{*}$}

Patienten mit einem medikamentös therapierefraktären fulminanten Schub sollen dringlich operiert werden.

[Evidenzgrad: B, Konsensusstärke: starker Konsens, Empfehlungsstärke: $\uparrow \uparrow]$

\section{Erläuterung}

Die Diagnose des therapierefraktären fulminanten Schubes wird interdisziplinär gestellt. Radiologisch weist eine Kolondilatation von $6 \mathrm{~cm}$ auf ein toxisches Megakolon hin [261, 262]. Der therapierefraktäre fulminante Schub stellt dann eine dringliche Operationsindikation dar, wenn sich über einen Zeitraum von 72 Stunden durch konservative intensivmedizinische bzw. medikamentöse Behandlung keine substanzielle Verbesserung der Erkrankungsintensität erzielen lässt. Eine sinnvolle Alternative zu einer weiteren Steigerung der medikamentösen Therapie stellt die Operation dar. Es ist hierbei zu berücksichtigen, dass eine weitere Steigerung der medikamentösen Therapie mittels Cyclosporin, Tacrolimus oder anti TNF- $\alpha$-Antikörper die Operation in maximal 60\% der Fälle um 1 Jahr aufschieben kann [483]. Kinder mit einem fulminanten Schub einer Colitis ulcerosa sollen in ein Zentrum mit kindergastroenterologischer und chirurgischer Expertise verlegt werden. Das Krankheitsbild ist bei Kindern sehr selten und die klinischen Symptome sind anders als bei Erwachsenen. Hilfreich kann die Berechnung des PUCAI (pediatric ulcerative colitis activity index) sein [484]. Hierbei beinhaltet ein Wert höher 45 Punkte an Tag 3 oder ein Wert höher 70 an Tag 5 eine hohe Sensitivität und Spezifität für „Nicht-Ansprechen auf Steroide“ [220].

\section{Empfehlung 7.5}

Ein trotz Einsatz von Immunsuppressiva inkl. Biologika therapierefraktärer Verlauf sollte als absolute Operationsindikation operiert werden.

[Evidenzgrad: D, Konsensusstärke: Konsens, Empfehlungsstärke: $\uparrow \uparrow]$

\section{Erläuterung}

Nach Versagen einer intensivierten, konservativen Therapie ist die chirurgische Entfernung des Kolons indiziert. Die Operation kann je nach Dauer und Intensität der Vormedikation und der klinischen Symptomatik dreizeitig erfolgen. Extraintestinale Komplikationen sollen präoperativ ausgeschlossen werden. 


\section{Empfehlung 7.6}

Bei Patienten mit CU und Kolonstenose unklarer Dignität soll operiert werden.

[Evidenzgrad: C, Konsensusstärke: starker Konsens, Empfehlungsstärke: $\uparrow \uparrow$ ]

\section{Erläuterung}

Kolonstenosen bei der Colitis ulcerosa gehen in ca. ein Drittel der Fälle mit einem Karzinom einher. Eine definitive histologische Klärung ist wegen des submukösen Wachstums des Colitis-ulcerosa-assoziierten Karzinoms häufig schwierig. Daher sollte die Operation aufgrund einer Kolonstenose auch bei nicht eindeutig nachgewiesener Neoplasie erfolgen [485-487].

\section{Empfehlung 7.7}

Eine elektive Operation kann bei Patientenwunsch erfolgen. Dabei sind die Risiken der konservativen Behandlungsstrategien gegen die Risiken einer Operation abzuwägen.

[Evidenzgrad: D, Konsensusstärke: starker Konsens, Empfehlungsstärke: $\uparrow]$

\section{Erläuterung}

Die Operation stellt eine gute Alternative zu einer langjährigen medikamentösen Therapie dar. Im Langzeitverlauf lässt sich trotz aller möglichen Komplikationen nach restaurativer Proktokolektomie mit ileo-analem Pouch eine gute Lebensqualität für ca. 95\% aller Patienten erreichen [479, 480]. Insbesondere Patienten, die ein dauerhaftes Krankheitsgefühl beklagen, eine schlechte Medikamenten-Compliance haben oder unter Karzinomangst leiden, profitieren besonders von einer Operation [478, 479, 482]. Grundvoraussetzung ist ein intensives Beratungsgespräch mit einem in der Pouchchirurgie erfahrenen Viszeralchirurgen. Vor der Entscheidung für eine Operation sollen funktionelle Beschwerden ausgeschlossen werden.

\section{Empfehlung 7.8}

Kinder und Jugendliche mit Wachstumsstörungen unter adäquater Therapie nach Ausschluss anderer Ursachen und Konsultation eines Kindergastroenterologen sollen operiert werden.

[Evidenzgrad: D, Konsensusstärke: Konsens, Empfehlungsstärke: $\uparrow \uparrow]$

\section{Erläuterung}

Bei Kindern und Jugendlichen mit Colitis ulcerosa sind Wachstumsstörungen sehr viel seltener als beim Morbus Crohn und sind dann Folge einer anhaltenden Entzündungsaktivität oder einer zu langen und zu hoch dosierten Steroidbehandlung, die unbedingt vermieden werden muss. Andere Ursachen einer Wachstumsstörung (z.B. Zöliakie, Wachstumshormonmangel, konstitutionelle Wachstumsverzögerung) müssen präoperativ ausgeschlossen werden. Die medikamentöse Behandlung und die Operationsindikation soll durch einen Kindergastroenterologen mitbeurteilt werden. Ein Aufholwachstum nach einer Operation ist nur bei präpubertären Kindern oder Kindern in frühen Pubertätsstadien zu erwarten, sodass trotz der schlechten Evidenzlage ein starke Empfehlung gerechtfertigt erscheint [488].

\section{Empfehlung 7.9*}

Bei erhöhtem perioperativem Risiko sollte die Proktokolektomie dreizeitig operiert werden.

[Evidenzgrad: D, Konsensusstärke: Konsens, Empfehlungsstärke: $\uparrow]$

\section{Erläuterung}

Die Durchführung der Operation in drei Schritten beinhaltet 1 . die subtotale Kolektomie mit endständigem Ileostoma, 2. die Restproktomukosektomie mit ileoanaler Pouchanlage und doppelläufigem Ileostoma und 3. die Ileostomarückverlagerung. Das dreizeitige Operationsverfahren bei erhöhtem perioperativem Risiko geht insgesamt mit einer geringeren Rate an Komplikationen einher als das ein- bzw. zweizeitige Verfahren [370, 371, 376, 489, 490]. Ein erhöhtes präoperatives Risiko besteht bei langfristiger Steroidtherapie, Therapie mit Biologika, Immunsuppression, Mangelernährung (siehe Statement 6.2) und bei etwaigen Begleiterkrankungen. Die Einnahme von mehr als $20 \mathrm{mg}$ Prednisolon über einen Zeitraum von mehr als 6 Wochen geht mit einer erhöhten Komplikationsrate der operativen Therapie einher. Nach Möglichkeit sollte die Steroiddosis präoperativ reduziert werden und gleichzeitig das Auftreten eines Steroidentzugs-Syndroms vermieden werden. Bei Kindern ist die entsprechende Dosis bei $15 \mathrm{mg} / \mathrm{m}^{2}$ KOF (bzw. 0,5 mg/kg) anzusetzen. In einigen Studien weisen Patienten unter Therapie mit anti-TNF- $\alpha$-Antikörpern ein erhöhtes Risiko für postoperative Komplikationen auf. Zusätzlich ist die dreizeitige Operation bei Patienten unter Therapie mit anti-TNF- $\alpha$-Antikörpern häufiger als bei Patienten ohne Therapie mit Biologika. Da keine prospektiv randomisierte Studie vorliegt, ist nicht eindeutig geklärt, ob die erhöhte Komplikationsrate evtl. durch die schwerere Grunderkrankung der dann mit Biologika behandelten Patienten bedingt ist [371, 374, 376].

In einigen Studien wurde eine erhöhte Anzahl von operativen Komplikationen (z.B. Insuffizienz von Anastomose oder Pouch) sowie septische Komplikationen beobachtet. Die kontinuierliche Einnahme von Azathioprin ist nicht mit einem erhöhten Risiko für postoperative Komplikationen behaftet. Bezüglich einer immunsuppressiven Therapie mit Calcineurininhibitoren zeigte eine Fallserie bei Kindern keine Unterschiede hinsichtlich postoperativer Komplikationen mit oder ohne präoperativer Therapie mit Calcineurininhibitoren [491].

\section{Empfehlung 7.10}

Bei Mangelernährung (hohes metabolisches Risiko) soll vor elektiver Operation präoperativ eine gezielte Ernährungstherapie für mindestens 7 Tage erfolgen.

[Evidenzgrad: B, Konsensusstärke: Konsens, Empfehlungsstärke: $\uparrow \uparrow]$

\section{Erläuterung}

Mangelernährung ist in der Chirurgie als Risikofaktor für das Auftreten von perioperativen Komplikationen sowohl retroals auch prospektiv gut belegt. Dies schließt Patienten mit Colitis ulcerosa mit ein [498-500]. In einer Kohortenstudie der Veterans Administration wurden bei 87078 chirurgischen Patienten (Ausschluss Herzchirurgie) als Hauptrisikofaktoren für die postoperative 30-Tage-Letalität der präoperative Serum-Albumin-Spiegel, die American Society of Anesthesia Class des 
Operationsrisikos (ASA) und die Notwendigkeit einer Notfalloperation identifiziert. Auch das Vorliegen eines Gewichtsverlusts von mehr als $10 \%$ war ein signifikanter prädiktiver Faktor. In einer prospektiven Erhebung an 5031 chirurgischen Patienten (Ausschluss Kardiochirurgie) der Veterans Administration von 1995-2000 wurden präoperative Risikofaktoren für das Entstehen einer Infektion im Operationsgebiet evaluiert. Hierbei zeigten sich Diabetes mellitus und Mangelernährung als unabhängige Faktoren [492].

Nach den S3-Leitlinien der Europäischen Gesellschaft für Parenterale und Enterale Ernährung (ESPEN) ist die Verschiebung einer Operation zur Durchführung einer gezielten künstlichen Ernährung für eine Dauer von 7-14 Tagen nur bei hohem ernährungsmedizinischem Risiko gerechtfertigt.

Nach der ESPEN-Definition liegt beim Eintreten eines oder mehrerer Kriterien eine schwere Mangelernährung vor:

- Gewichtsverlust $>10-15 \%$

- $\mathrm{BMI}<18,5 \mathrm{~kg} / \mathrm{m}^{2}$

- Serum-Albumin $<30 \mathrm{~g} / \mathrm{L}$ (keine Einschränkung der Leber- und Nierenfunktion)

Bei gegebener Indikation zur präoperativen Ernährung sollte die enterale Zufuhr über Trink- und Sondennahrung vorgezogen werden. Die enterale Ernährung ist zur Vermeidung nosokomialer Infektionen möglichst prästationär durchzuführen. Die parenterale Applikation sollte Patienten mit hoher entzündlicher Aktivität im Kolon und intestinaler Unverträglichkeit vorbehalten bleiben [489, 490, 492-496].

\section{Empfehlung 7.11}

Bei der dreizeitigen Proktokolektomie sollte die Kolektomie bis zum rektosigmoidalen Übergang erfolgen.

[Evidenzgrad: D, Konsensusstärke: Konsens, Empfehlungsstärke: $\uparrow$ ]

\section{Erläuterung}

Die hohe Komplikationsrate unter intensivierter medikamentöser Therapie macht das komplikationsärmere dreizeitige Vorgehen erforderlich. Da die Entfernung des Rektums der risikoreichste und für den Patienten am meisten belastende Anteil der Operation ist, wird im ersten Schritt eine subtotale Kolektomie mit Anlage eines endständigen Ileostomas durchgeführt. Jedoch sollte berücksichtig werden, dass möglichst viel entzündungstragendes Kolon entfernt wird. Mit einer Resektion bis zum rektosigmoidalen Übergang sind in der Regel diese beiden Forderungen erfüllt und die Restproktektomie technisch leicht möglich.

Der Verschluss des Rektumstumpfs erfolgt als Blindverschluss nach Hartmann oder unter Belassung des Colon sigmoideum als Ausleitung desselbigen als Schleimfistel im linken Unterbauch. Durch die letztere Variante kann eine etwaige Insuffizienz des Hartmann-Stumpfs vermieden werden und es besteht die Möglichkeit, in der Zeit zwischen der zweiten und dritten Operation topisch über die Sigmaschleimfistel mit Kortikoiden oder 5-ASA zu therapieren.

\section{Empfehlung 7.12}

Bei der ileoanalen Pouchanlage soll die belassene Rektummukosa nicht länger als $2 \mathrm{~cm}$ sein.

[Evidenzgrad: D, Konsensusstärke: starker Konsens, Empfehlungsstärke: $\uparrow \uparrow]$

\section{Erläuterung}

Die letzten $2 \mathrm{~cm}$ oral der Linea dentata sind funktionell bedeutend und somit für die Lebensqualität der Patienten einflussreich. Innerhalb der letzten $2 \mathrm{~cm}$ oral der Linea dentata befindet sich die anale Transitionalzone, die eine große Rolle für den Erhalt der Nachtkontinenz spielt. Bei der Operationsstrategie muss jedoch berücksichtigt werden, dass postoperativ das Risiko zur Ausbildung einer Rezidiv-/Persistenz-Proctitis ulcerosa besteht. Da die Erkrankungsschwere, das Beschwerdeausmaß und das Risiko für ein Rezidiv bzw. eine Persistenz mit der Länge der verbliebenen Rektummukosa exponentiell korreliert, sollte die Länge der belassenen Rektummukosa $2 \mathrm{~cm}$ nicht übersteigen. Sollte innerhalb der belassenen Rektum-Mukosa eine Proctitis ulcerosa entstehen, besteht die Möglichkeit einer topischen Therapie mit 5-ASA oder als Ultima Ratio eine sekundäre Mukosektomie [497-499].

Hinsichtlich der Anastomosenart scheint die Stapler-Anastomose unter Belassung der analen Transitionalzone für die Nachtkontinenz besser zu sein, wenn auch prospektiv randomisierte Studien zu dieser Fragestellung fehlen. Nichtsdestotrotz muss jeder Chirurg, der eine restaurative Proktokolektomie durchführt, in der Lage sein, bei technischem Versagen einer Stapleranastomose eine transanale Handnaht durchzuführen.

\section{Empfehlung 7.13}

Bei Proktitis ulcerosa in belassener Rektummukosa kann topisches 5-ASA eingesetzt werden. Alternativ kann eine sekundäre transanale Mukosektomie bei starken Symptomen erfolgreich sein. [Evidenzgrad: D, Konsensusstärke: starker Konsens, Empfehlungsstärke: $\uparrow$ ]

\section{Erläuterung}

Die Primärtherapie einer Proktitis ulcerosa in belassener Rektummukosa soll unter topischer Anwendung der Substanzen erfolgen. Hierbei sind Suppositorien besser geeignet als Einläufe [500]. Topisches 5-ASA ist geeignet eine Remission bei einer aktiven Proktitis ulcerosa zu induzieren [183]. Topisches 5-ASA ist hierbei mindestens doppelt so wirksam wie topische Steroide [299]. Alternativ zur medikamentösen Therapie, insbesondere bei Nachweis intraepithelialer Neoplasien, kann eine sekundäre transanale Mukosektomie erfolgen [497, 501]. Die operative Therapie ist mit den Patienten hinsichtlich Beschwerdelinderung, Funktionalität und Entartungsrisiko zu diskutieren.

\section{Empfehlung 7.14*}

Unter der Indikation einer intraepithelialen Neoplasie oder eines manifesten Karzinoms soll eine komplette Mukosektomie mit Anastomose an der Linea dentata durchgeführt werden.

[Evidenzgrad: D, Konsensusstärke: starker Konsens, Empfehlungsstärke: $\uparrow \uparrow$ ]

\section{Erläuterung}

In der Literatur sind ca. 50 Fälle von Pouch-Karzinomen beschrieben. Die Mehrzahl dieser publizierten Pouch-KarzinomFälle geht auf eine belassene Rektum-Mukosa zurück. Daher sollte bei bestehender Risikokonstellation in Form von intraepithelialen Neoplasien respektive eines manifesten Karzinoms immer radikal die gesamte Rektum-Mukosa entfernt werden [486, 502-504]. 


\section{Empfehlung 7.15*}

Pouchchirurgie soll nur in dafür spezialisierten Zentren durchgeführt werden.

[Evidenzgrad: D, Konsensusstärke: starker Konsens, Empfehlungsstärke: $\uparrow \uparrow$ ]

\section{Erläuterung}

Die restaurative Proktokolektomie mit Ileum-J-Pouch-analer Rekonstruktion ist ein komplexer Eingriff, der technisch sowie in der prä- und postoperativen Phase eine langjährige Erfahrung und hohe Expertise erfordert. Für vergleichbar komplexe Eingriffe (Pankreas, Ösophagus) bestehen seit einigen Jahren Mindestmengenregelungen, die in der Regel Mindestmengen von zehn Eingriffen pro Jahr umfassen. Für die ileoanale Pouchchirurgie ist ebenfalls in einer Studie ausgewiesen, dass bei einer Eingriffszahl von über 10 pro Zentrum pro Jahr die Komplikationsrate signifikant absinkt [505].

\section{Empfehlung 7.16}

Patienten mit einer chronischen Pouchitis oder nach Colitis-ulcerosa-assoziiertem Karzinom oder intraepithelialer Neoplasie sollten jährlich endoskopisch überwacht werden.

[Evidenzgrad: D, Konsensusstärke: starker Konsens, Empfehlungsstärke: $\uparrow$ ]

\section{Erläuterung}

Selbst nach makroskopisch komplett durchgeführter Mukosektomie besteht ein Restrisiko für kleinste verbliebene Schleimhautinseln. Diese Schleimhautinseln können der Ursprung einer intraepithelialen Neoplasie oder eines Karzinoms darstellen. Aus diesem Grund sollte die Patientengruppe, die aufgrund einer intraepithelialen Neoplasie oder eines Karzinoms operiert worden ist, jährlich nachgesorgt werden. Es bestehen zwar keine sicheren Hinweise darauf, dass eine chronische Pouchitis langfristig eine maligne Transformation bedingt, dennoch erscheint es sinnvoll, dass auch für diese Situation postoperativ eine jährliche endoskopische Überwachung erfolgt. Daneben ist für diese besondere Patientengruppe ein enger Arztkontakt zur Verbesserung der Lebensqualität bei vorhandenem Pouch erforderlich [504].

\section{Empfehlung 7.17}

Die Kolektomie mit ileorektaler Anastomose kann nur für ausgewählte Konstellationen wie z. B. bei dringendem Kinderwunsch empfohlen werden.

[Evidenzgrad: D, Konsensusstärke: starker Konsens, Empfehlungsstärke: $\downarrow]$

\section{Erläuterung}

Proktokolektomien mit ileoanaler Pouchanlage führen sowohl bei Frauen als auch bei Männern zu erhöhter Infertilität. Daher sollte Patienten mit Kinderwunsch und bestehender Operationsindikation eine Kolektomie mit Ileorektostomie angeboten werden. Grundvoraussetzung für dieses Vorgehen ist jedoch, dass das belassene Rektum weitestgehend entzündungsfrei und damit auch anastomosefähig ist. Eine sekundäre Rektumresektion mit Proktektomie und IPAA kann angestrebt werden. Aufgrund der Seltenheit dieser Operation bei Kindern soll ein spezialisiertes viszeralchirurgisches Zentrum, ggf. in Zusam- menarbeit mit Kinderchirurgen, diese Operation bei Kindern durchführen. Die postoperative Betreuung soll in jedem Fall durch oder in enger Kooperation mit Kindergastroenterologen erfolgen.

\section{Empfehlung 7.18}

Das kontinente Ileostoma nach Kock kann als mögliche Alternative für besondere Fälle angeboten werden.

[Evidenzgrad: D, Konsensusstärke: Konsens, Empfehlungsstärke: $\uparrow$ ]

\section{Erläuterung}

Das kontinente Ileostoma nach Kock ist ein technisch komplexes Operationsverfahren mit hoher Komplikationsrate hinsichtlich Funktion. Durch das kontinente Ileostoma nach Kock kann die Lebensqualität der Patienten im Vergleich zu einem herkömmlichen, nicht kontinenten Ileostoma gesteigert und das Körperempfinden verbessert werden [506-509]. Bei Pouchversagen kann, bei ausgeprägtem Wunsch des Patienten, eine Umwandlung in ein kontinentes Ileostoma nach Kock erfolgen.

\section{Empfehlung 7.19}

Bei belassenem Rektum unter ileorektaler Anastomose oder bei endständigem lleostoma mit Rektumblindverschluss nach Hartmann sollte eine jährliche endoskopische Kontrolle des belassenen Rektums mit Stufenbiopsien erfolgen.

[Evidenzgrad: D, Konsensusstärke: Konsens, Empfehlungsstärke: $\uparrow]$

\section{Erläuterung}

Da durch das Belassen des Rektums ein Entartungsrisiko der Rektumschleimhaut weiterhin besteht, ist eine endoskopische Kontrolle mit Biopsien indiziert. Eine sekundäre restaurative Proktokolektomie mit IPAA soll mit dem Patienten diskutiert werden. Die Literatur beinhaltet einzelne Berichte über langjähriges Bestehen von Ileorektostomien nach Kolektomien in einer Notfallsituation [510, 511]. Dies stellt jedoch nicht den Regelfall dar.

\section{Empfehlung 7.20}

Die laparoskopische restaurative Proktokolektomie kann als gleichwertige Alternative zur offenen Operation angeboten werden.

[Evidenzgrad: B, Konsensusstärke: starker Konsens, Empfehlungsstärke: $\uparrow$ ]

\section{Erläuterung}

Die laparoskopische restaurative Proktokolektomie mit IPAA ist eine sicher durchführbare Operationsmethode unter der Voraussetzung einer entsprechenden Erfahrung des Zentrums. Bisher konnten lediglich kosmetische Vorteile gegenüber einer offenen restaurativen Proktokolektomie mit IPAA gezeigt werden [512 -514]. Die handassistierte laparoskopische restaurative Proktokolektomie mit IPAA stellt eine weitere Alternative dar. Bezüglich der kurz- bzw. langfristigen Ergebnisse gibt es keine nachgewiesenen Unterschiede im Vergleich der verschiedenen Operationsmethoden. 


\section{Empfehlung 7.21}

Bei Colitis indeterminata ohne anorektales Fistelleiden und entsprechender Operationsindikation kann eine restaurative Proktokolektomie unter Aufklärung mit den damit verbundenen Risiken dem Patienten angeboten werden.

[Evidenzgrad: D

Konsensusstärke starker Konsens, Empfehlungsstärke: $\uparrow$ ]

\section{Erläuterung}

Bei ungefähr 7\% der Patienten mit einer Colitis kann keine exakte Diagnose gestellt werden, um Morbus Crohn von Colitis ulcerosa zu unterscheiden [515]. In der Literatur finden sich Berichte über ein schlechteres Langzeitergebnis nach restaurativer Proktokolektomie mit IPAA bei Patienten mit Colitis indeterminata. Diesen stehen jedoch Berichte gegenüber, in denen Patienten mit Colitis indeterminata kein schlechteres Langzeitergebnis haben als Patienten mit Colitis ulcerosa [516, 517]. Die sekundäre Diagnose eines Morbus Crohn nach stattgehabter restaurativer Proktokolektomie mit IPAA ist oftmals mit Komplikationen, insbesondere einem Pouchversagens, behaftet. Bezüglich der Rate an Pouchversagen gibt es auch Berichte über unauffällige Verläufe [518]. Trotz dieser widersprüchlichen Datenlage kann eine restaurative Proktokolektomie mit IPAA - nach ausführlicher Diskussion mit dem Patienten durchgeführt werden. Bei Kindern und Jugendlichen liegt der Anteil der Patienten mit Colitis indeterminata bei bis zu $22 \%$ [519]. Im Verlauf kann bei einem Großteil der Patienten eine diagnostische Zuordnung zu Colitis ulcerosa oder Morbus Crohn gelingen, sodass bei Kindern mit operationsbedürftiger Colitis indeterminata ein dreizeitiges Vorgehen sinnvoll ist.

\section{Pouchitis}

\section{Empfehlung 7.22}

Die Diagnose Pouchitis soll unter der Berücksichtigung der Parameter Klinik, Endoskopie und Histologie erfolgen.

[Evidenzgrad: C, Konsensusstärke: starker Konsens, Empfehlungsstärke: $\uparrow \uparrow$ ]

\section{Erläuterung}

Die Pouchitis wird als Entzündung im Pouch nach Ausschluss operativer Komplikationen oder anderer sekundärer Ursachen definiert. Es sollten hierbei verschiedene Verlaufsformen unterschieden werden. Die Einteilung soll anhand des zeitlichen Verlaufs in akute, akut-rezidivierende und chronisch atrophische Pouchitis erfolgen. Die Diagnose einer akuten Pouchitis wird auf der Basis der klinischen Symptomatik (Stuhlfrequenz, Blutung, Fieber, Schmerzen), ergänzt durch Endoskopie (Rötung, Ödem, Erosionen, Ulzerationen, Spontaneinblutungen, Fibrinbeläge), Histologie (Ulzerationen, Kryptenabszesse, Infiltrationen durch Granulozyten ...) und der klinischen, insbesondere rektal-digitalen Untersuchung gestellt [520]. Die akut-rezidivierende Pouchitis ist durch wiederholt auftretende akute Schübe einer Pouchitis gekennzeichnet. Die Diagnose einer chronischen Pouchitis ergibt sich durch eine entzündliche Reaktion im Pouch, deren Klinik und endoskopischer/histologischer Befund über mehr als 3 Monate anhält. Das Risiko einer akuten Pouchitis liegt bei Patienten nach restaurativer Proktokolektomie mit IPAA bei Colitis ulcerosa bei etwa $30 \%$ in den ersten zwei Jahren nach Operation. Im Laufe der Nachbeobachtungs- zeit steigt das Risiko langsam auf bis über 50\% an [521-527]. Bei ungefähr 5\% der Patienten mit einer Pouchitis geht die akute Verlaufsform in eine chronische über. Risikofaktoren für eine Pouchitis sind extraintestinale Manifestationen. Hier ist insbesondere die PSC zu erwähnen [527-531]. Zusätzlich gehen anhaltend hohe Entzündungsaktivität und Backwashileitis mit einer erhöhten Rate an Pouchitis einher. Die Parameter zur Diagnose der akuten Pouchitis werden durch den Pouchitis Disease Activity Index (PDAI) zusammengefasst [520]. Wenn sich trotz entsprechender klinischer Symptomatik nach endoskopischen und histologischen Kriterien kein Anhalt für eine Pouchitis findet und andere Krankheiten ausgeschlossen wurden, kann die Diagnose eines irritablen Pouchsyndroms gestellt werden [532].

\section{Empfehlung 7.23}

Bei einer chronischen Pouchitis soll eine chirurgisch behandelbare Ursache ausgeschlossen werden.

[Evidenzgrad: D, Konsensusstärke: starker Konsens, Empfehlungsstärke: $\uparrow \uparrow$ ]

\section{Erläuterung}

Bei Diagnosestellung „Pouchitis“ sind Untersuchungen zum Ausschluss von sekundären Pouchitisformen empfohlen. Zum Ausschluss einer chirurgischen Ursache einer Pouchitis sind Computertomografie, Kernspintomografie, Kontrasteinlauf und Endosonografie notwendig und geeignet [533-536]. Ggf. sind diese Untersuchungsmethoden im Verlauf zu wiederholen. Es sind Fisteln, Abszesse, Anastomoseninsuffizienzen und Ischämien auszuschließen.

\section{Empfehlung 7.24}

Als Primärtherapie der akuten Pouchitis soll Ciprofloxacin oder Metronidazol eingesetzt werden.

[Evidenzgrad: A, Konsensusstärke: Konsens, Empfehlungsstärke: $\uparrow \uparrow]$

\section{Erläuterung}

Es gibt mehrere randomisierte kontrollierte Studien zur medikamentösen Therapie der Pouchitis [537-539]. Die Empfehlung zur Therapie der akuten Pouchitis mit Antibiotika (Metronidazol, Ciprofloxazin) wird durch insgesamt 3 offene, unkontrollierte Studien und eine randomisierte nicht verblindete Studie gestützt [540-543]. Zu Dosis und Dauer der Antibiotika-Therapie liegen keine gesicherten Daten vor, sie müssen individuell getestet werden. Üblich ist eine orale Therapie mit Metronidazol 2-3×400 mg oder Ciprofloxazin $2 \times 250$ bis $2 \times 500 \mathrm{mg} / \mathrm{d}$ für $1-2$ Wochen. Bei Unverträglichkeit von oralem Metronidazol stellt topisch angewendetes Metronidazol eine Alternative dar [544]. Bei fehlendem Ansprechen oder Unverträglichkeit einer Therapie mit Metronidazol ist eine Therapie mit Ciprofloxazin indiziert [540].

\section{Empfehlung 7.25}

Bei einer chronischen Pouchitis kann eine kombinierte antibiotische Therapie eingesetzt werden.

[Evidenzgrad: C, Konsensusstärke: starker Konsens, Empfehlungsstärke: $\uparrow$ ] 


\section{Erläuterung}

Bei fehlendem Ansprechen einer Monotherapie mit Metronidazol oder Ciprofloxazin kann eine orale Kombinationstherapie aus Ciprofloxazin und Metronidazol oder eine orale Kombinationstherapie aus Ciprofloxazin und Rifaximin eingesetzt werden $[545,546]$.

\section{Empfehlung 7.26}

Zum Remissionserhalt kann eine probiotische Therapie eingesetzt werden.

[Evidenzgrad: A, Konsensusstärke: Konsens, Empfehlungsstärke: $\uparrow]$

\section{Erläuterung}

Bisher liegt eine prospektiv randomisierte Studie zur Primärprophylaxe der Pouchitis mit dem Probiotikagemisch VSL\#3 vor [547, 548]. Die Effektivität konnte dabei nachgewiesen werden. Alternative Möglichkeiten der Remissionserhaltung sollen mit den Patienten diskutiert werden.

\section{Empfehlung 7.27}

Nach Pouchanlage sollte eine jährliche Kontrolluntersuchung erfolgen.

[Evidenzgrad: D, Konsensusstärke: Konsens, Empfehlungsstärke: $\uparrow \uparrow]$

\section{Erläuterung}

Diese Empfehlung stützt sich nicht auf spezifische Studien in der Literatur. Basierend auf den Erfahrungen der Überwachungsstrategien vor restaurativer Proktokolektomie mit IPAA ist eine jährliche endoskopische Kontrolluntersuchung sinnvoll, obwohl das Pouch-Karzinom eine seltene Entität ist. Patienten mit einem Pouch-Karzinom hatten in der Regel bereits ein Karzinom im Kolon oder Rektum, welches zur restaurativen Proktokolektomie mit IPAA geführt hat. Pouch-Karzinome entstehen in der Regel aus verbliebener Schleimhaut des anorektalen Übergangs, sodass dies eine Indikation zur kompletten Mukosektomie im Rahmen der restaurativen Proktokolektomie mit IPAA darstellt [549, 550].

\section{Kapitel 8: Karzinom-Prophylaxe \\ $\boldsymbol{\nabla}$}

\section{Risikokonstellation}

\section{Statement $\mathbf{8 . 1}$}

Das kolorektale Karzinomrisiko ist bei Patienten mit Colitis ulcerosa im Vergleich zur Normalbevölkerung erhöht.

[Evidenzgrad: B, Konsensusstärke: starker Konsens]

\section{Statement $\mathbf{8 . 2}$}

Das Risiko ist hoch bei ausgedehnter Kolitis, erhöht bei Linksseitenkolitis und nicht eindeutig erhöht bei der Proktitis ulcerosa.

[Evidenzgrad: B, Konsensusstärke: starker Konsens]

\section{Statement $\mathbf{8 . 3}$}

Das Risiko steigt mit der Dauer der Erkrankung an, korreliert positiv mit der Ausprägung der entzündlichen Aktivität im Verlauf und ist bei einer zusätzlich bestehenden PSC noch stärker erhöht. [Evidenzgrad: EK2a/2b, EG B, Konsensusstärke: starker Konsens]

\section{Erläuterung}

Patienten mit CU zeigen in zahlreichen Studien ein erhöhtes Risiko für ein kolorektales Karzinom, wobei unterschiedliche Angaben über die Höhe des Risikos zu finden sind. Während frühere Studien aus Zentren - zusammengefasst in einer Metaanalyse mit mehr als 50000 Patienten aus 116 Studien - das Risiko für ein kolorektales Karzinom bei Patienten mit Colitis ulcerosa mit 18\% nach 30 Jahren bezifferten [551], zeigten populationsbasierte Studien der letzten Jahre nur leicht erhöhte Karzinomraten [552-554]. Lediglich in Dänemark fand sich keine erhöhte Inzidenz, möglicherweise wegen einer höheren Kolektomierate [554]. Auch wiesen Fallserien zur Überwachungskoloskopie in den letzten Jahren niedrigere Karzinomraten auf. Die Ursachen für dieses Phänomen sind unklar und könnten in einer schlechteren Behandlung oder einem Selektionsbias in früheren Studien beruhen. Die Häufigkeit korreliert mit der Erkrankungsdauer, der Ausdehnung und der entzündlichen Aktivität/Pseudopolypen [14, 551, 555-557]. So lag die kumulative Karzinomrate in der Metaanalyse von Eaden nach 30 Jahren bei $18 \%$ und in der Fallserie des St. Mark's Hospitals bei $7,6 \%$.

Eine besondere Risikogruppe sind Patienten, die gleichzeitig eine primär sklerosierende Cholangitis haben. Eine Metaanalyse ergab ein fünffach erhöhtes Karzinomrisiko, wobei häufiger rechtsseitige Karzinome auftreten [15, 417].

In manchen Studien wird auch berichtet, dass ein Kolonkarzinom in der Familienanamnese, das Vorhandensein einer Backwash-Ileitis und eine Kolitis-Erstmanifestation im Kindes- und Jugendalter Risikofaktoren darstellen [551, 556, 558]. Die Datenlage diesbez. ist jedoch uneinheitlich.

\section{Überwachungskoloskopie, zeitliche Strategie}

\section{Empfehlung 8.4*}

Da die colitisassoziierte Kolonkarzinommortalität durch eine endoskopische Überwachung gesenkt werden kann, sollen regelmäßige Überwachungskoloskopien erfolgen.

[Evidenzgrad: B, Konsensusstärke: Konsens, Empfehlungsstärke: $\uparrow \uparrow]$

\section{Empfehlung $\mathbf{8 . 5}$}

Zur Festlegung der Überwachungsstrategie soll bei allen CU-Patienten unabhängig von der Krankheitsaktivität eine Kontrollkoloskopie zur Erfassung des Befallsmusters spätestens 8 Jahre nach Beginn der Symptomatik erfolgen.

[Evidenzgrad: EK2a, EGC., Konsensusstärke: Konsens, Empfehlungsstärke: $\uparrow \uparrow$ ] 


\section{Empfehlung $\mathbf{8 . 6}$}

Die Überwachungskoloskopien sollten dann bei ausgedehnter CU ab dem 8. Jahr und bei linksseitiger oder distaler CU ab dem 15. Jahr nach Erstmanifestation 1 - 2 jährlich erfolgen.

[Evidenzgrad: C, Konsensusstärke: Konsens, Empfehlungsstärke: $\uparrow \uparrow]$

\section{Empfehlung $\mathbf{8 . 7}$}

Wenn gleichzeitig eine PSC besteht, sollen die Überwachungskoloskopien unabhängig von der Krankheitsaktivität und Ausdehnung der CU ab dem Zeitpunkt der PSC-Diagnosestellung jährlich erfolgen.

[Evidenzgrad: C., Konsensusstärke: Konsens, Empfehlungsstärke: $\uparrow \uparrow]$

\section{Empfehlung $\mathbf{8 . 8}$}

Nach subtotaler Kolektomie sollen in Analogie die gleichen endoskopischen Überwachungsstrategien wie bei einer CU ohne Resektion erfolgen.

[Evidenzgrad: C, Konsensusstärke: starker Konsens, Empfehlungsstärke: $\uparrow \uparrow]$

\section{Erläuterung}

Eine Metaanalyse von Collins aus dem Jahr 2006 fasst die direkte und indirekte Evidenz zu Überwachungskoloskopien bei Colitis ulcerosa zusammen. Die drei identifizierten Fallkontrollstudien konnten keine statistisch signifikante Reduktion des Risikos, an einem kolorektalen Karzinom zu erkranken, zeigen. Dabei ist anzumerken, dass es sich um kleine Studien mit teilweise aus heutiger Sicht inadäquatem Koloskopiestandard handelte. Dahingegen konnte die Metaanalyse deutliche indirekte Evidenz identifizieren, dass Überwachungskoloskopien sehr wahrscheinlich das Risiko, an einem Colitis-assoziierten Kolonkarzinom zu versterben, vermindern können, und gleichzeitig kosteneffektiv sind [559]. Dies basiert darauf, dass Colitis-assoziierte Kolonkarzinome früher erkannt werden, obwohl dennoch zwischen Überwachungsuntersuchungen Intervallkarzinome auftreten können [557]. Das Karzinomrisiko steigt mit der Krankheitsdauer und der Krankheitsausdehnung an, weshalb bei Patienten mit einer Pancolitis die regelmäßige Überwachung früher als bei Patienten mit einer distalen Colitis beginnen sollte. Da sich aus einer initial als distale Colitis beschriebenen Entzündung auch ohne klinische Hinweise eine Pancolitis entwickeln kann, sollte eine Kontrollkoloskopie innerhalb von 8 Jahren nach den ersten Krankheitssymptomen durchgeführt werden, um die Ausdehnung zu überprüfen und danach das Überwachungsvorgehen festlegen zu können. Eine niederländische Studie weist darauf hin, dass bereits bis zu $22 \%$ der Patienten vor dem Start der bisher empfohlenen Überwachungskoloskopien Colitis-assoziierte Kolonkarzinome entwickelten [560]. Wenn Patienten mit PSC herausgerechnet wurden - die ab Diagnosestellung überwacht werden sollten - reduziert sich die Rate der „entgangenen“ Karzinome auf ca. $15 \%$.

Das Überwachungsintervall zwischen den Untersuchungen sollte höchstens 2 Jahre betragen, weil in diesem Zeitraum bereits Intervallkarzinome auftreten können [168, 561]. Da bei einer Proktitis das Karzinomrisiko allenfalls minimal erhöht ist, muss - bei Fehlen sonstiger Risikofaktoren - keine regelhafte Überwachung erfolgen.

Da das Karzinomrisiko bei Patienten mit CU und einer PSC fünffach erhöht ist [15] und Karzinome gehäuft rechtsseitig auftreten [417], sollte bei diesen Patienten eine Überwachung ab Diagnosestellung unabhängig vom Befall jährlich erfolgen.

Nach subtotaler Kolektomie können im verbliebenen Darm, wie auch nach restaurativer Proktokolektomie im Pouch bzw. je nach Operationstechnik im Bereich der verbliebenen Kolonmukosa distal der Anastomose, Karzinome auftreten [562], sodass eine regelhafte Überwachung des verbliebenen Kolons bzw. des Pouches erfolgen sollte.

\section{Überwachungskoloskopie, Durchführungsbedingungen}

\section{Empfehlung $\mathbf{8 . 9}$}

Der geplante Zeitraum der Überwachungskoloskopie soll an die besondere Situation angepasst und die Rückzugszeit bei der Überwachungskoloskopie soll ausreichend lang sein.

[Evidenzgrad: C, Konsensusstärke: Konsens, Empfehlungsstärke: $\uparrow \uparrow]$

\section{Empfehlung $\mathbf{8 . 1 0}$}

Überwachungskoloskopie sollen in einem sauberen Darm durchgeführt werden. Bei Restverschmutzung ist eine Wiederholung erforderlich.

[Evidenzgrad: D, Konsensusstärke: Konsens, Empfehlungsstärke: $\uparrow \uparrow]$

\section{Empfehlung $\mathbf{8 . 1 1}$}

Biopsien sollen in der Remissionsphase gewonnen werden, da die histomorphologische Abgrenzung von entzündlichen gegenüber neoplastischen Veränderungen schwierig sein kann.

[Evidenzgrad: D, Konsensusstärke: starker Konsens, Empfehlungsstärke: $\uparrow \uparrow$ ]

\section{Erläuterung}

Überwachungskoloskopien zielen auf die Detektion von Neoplasien mit hoher Sensitivität und Spezifität. Dazu gehört, dass der Darm keine wesentlichen entzündlichen Veränderungen aufweist, die als intraepitheliale Neoplasien histologisch fehlgedeutet werden könnten. In Analogie zu Vorsorgekoloskopien in der Normalbevölkerung ist davon auszugehen, dass die Güte der Vorbereitung die Detektionsrate von Läsionen deutlich erhöht [563]. Ebenso besteht eine Korrelation zwischen der Rückzugszeit und der Detektionsrate von Neoplasien [564].

\section{Empfehlung $\mathbf{8 . 1 2}$}

Gezielte Biopsien sollen aus allen endoskopisch suspekten Läsionen entnommen werden.

[Evidenzgrad: A, Konsensusstärke: starker Konsens, Empfehlungsstärke: $\uparrow \uparrow$ ] 


\section{Empfehlung 8.13*}

Bei einer Überwachungskoloskopie bei CU sollen sowohl ungezielte Biopsien (mindestens 4 alle $10 \mathrm{~cm}$ ) als auch gezielte Biopsien aus allen auffälligen Arealen entnommen werden.

[Evidenzgrad: B, Konsensusstärke: Konsens, Empfehlungsstärke: $\uparrow \uparrow]$

\section{Empfehlung 8.14*}

Alternativ kann eine Chromoendoskopie mit gezielten Biopsien aus allen auffälligen Arealen erfolgen.

[Evidenzgrad: A, Konsensusstärke: Konsens, Empfehlungsstärke: $\uparrow]$

\section{Empfehlung $\mathbf{8 . 1 5}$}

Der Stellenwert der hochauflösenden virtuellen Chromoendoskopie (NBI, FICE, iScan) mit gezielten Biopsien ist nicht ausreichend definiert und soll deshalb nicht als alleinige Strategie verfolgt werden.

[Evidenzgrad: A, Konsensusstärke: Konsens, Empfehlungsstärke: $\downarrow \downarrow]$

\section{Erläuterung}

Mehrere Studien der letzten Jahre konnten zeigen, dass ein Großteil intraepithelialer Neoplasien mittels hochauflösender Endoskopie detektierbar ist, entweder als irreguläre Mukosa, Striktur oder Schleimhauterhabenheiten [123, 565, 566]. Von größter Bedeutung ist daher die gezielte Entnahme mehrerer Biopsien aus solchen Arealen. Da intraepitheliale Neoplasien aber auch makroskopisch unsichtbar sein können (ca. 20\%), sollte versucht werden, diese nachzuweisen [566]. Rubin et al. konnten in einem mathematischen Modell zeigen, dass mindestens 34 „blinde“ Biopsien benötigt werden, um ein 90\%iges Konfidenzintervall für die Detektion von Karzinomen zu erreichen, 64 Biopsien bedingen ein 95\%iges Konfidenzintervall [567]. Um eine über 90\%ige Sicherzeit in der Detektion zu erzielen, wird die Entnahme von 4 Biopsien alle $10 \mathrm{~cm}$ international empfohlen. Angesichts der häufigen Sichtbarkeit intraepithelialer Neoplasien (IEN) bei der hochauflösenden Endoskopie wird diese Empfehlung zur Entnahme von Zufallsbiopsien aber kontrovers diskutiert. So ergab eine Untersuchung zur Umsetzung der Empfehlung „Entnahme von 40-50 Zufallsbiopsien“ bei der Überwachungskoloskopie in Deutschland, dass nur 9,2\% der Überwachungskoloskopien in Deutschland entsprechend leitliniengerecht durchgeführt wurden [568]. Diese Problematik der geringen Akzeptanz der Empfehlung zu multiplen Zufallsbiopsien wird noch dadurch verstärkt, dass sich in verschiedenen Studien beim Vergleich von klassischer Koloskopie mit der Chromoendoskopie nur extrem selten bei den Zufallsbiopsien eine IEN fand (Kießlich 2003: 2 IEN bei 5098 Zufallsbiopsien [100] und Rutter 2004: 0 IEN bei 2906 Zufallsbiopsien [566]). Die aktuelle Studienlage reicht aber nicht aus, um ganz auf die Empfehlung von Zufallsbiopsien zu verzichten. Alternativ kann eine Chromoendoskopie (Indigokarmin oder Methylenblau) mit gezielten Biopsien durchgeführt werden. In zwei prospektiven, monozentrischen Studien unter Verwendung von Zoomkoloskopen konnten dadurch mehr Neoplasien als durch Weißlichtendoskopie mit ungezielten Biopsien entdeckt werden [100, 566]. Eine multizentrische
Arbeit aus New York fand ebenfalls mehr intraepitheliale Neoplasien mittels Chromoendoskopie [569]. Offen ist jedoch, wie viel Erfahrung der Untersucher für derartige Chromoendoskopien benötigt und ob die neueste Endoskopiegeneration mit Einsatz von HDI- bzw. HDTV-Qualität, nicht eine ähnlich hohe Detektionsrate von IEN erzielen könnte. Als mögliches Ersatzverfahren wurden physikalische oder virtuelle Chromoendoskopieverfahren (NBI, FICE, iScan) diskutiert, für die bisher keine belastbaren Daten bez. der Gleichwertigkeit in der IENDetektion zur Chromoendoskopie vorliegen. Die bisher einzige prospektive Studie hierzu ergab keinen Vorteil gegenüber Weißlicht [570].

Karzinome, IEN, ALM, DALM sowie Therapiekonsequenzen

\section{Empfehlung 8.16*}

Bei Nachweis von intraepithelialen Neoplasien (IEN) soll eine externe, unabhängige pathologische Zweitbeurteilung eingeholt werden.

[Evidenzgrad: A, Konsensusstärke: starker Konsens, Empfehlungsstärke: $\uparrow \uparrow$ ]

\section{Empfehlung $\mathbf{8 . 1 7}$}

Bei Vorliegen einer fraglichen IEN/Dysplasie soll eine endoskopische Kontrolle ggf. nach Intensivierung der antiinflammatorischen Therapie innerhalb von 3 Monaten durchgeführt werden.

[Evidenzgrad: B, Konsensusstärke: starker Konsens, Empfehlungsstärke: $\uparrow \uparrow]$

\section{Erläuterung}

Der Nachweis einer IEN/Dysplasie sowie deren Gradeinteilung ist für die Beurteilung des Kolonkarzinomrisikos bei Patienten mit CU von entscheidender Bedeutung. Anhand von Kolektomiepräparaten mit nachgewiesenem Karzinom fanden sich in postoperativ ungezielt entnommenen Quadrantenbiopsien IEN/Dysplasien in 74\% der Präparate; in Kolektomiepräparaten ohne Karzinom konnte in nur 26\% der Fälle eine IEN/Dysplasie in ungezielten Quadrantenbiopsien nachgewiesen werden [164]. Eine Metaanalyse zeigte, dass bei niedriggradigen IEN/ Dysplasien das Karzinomrisiko bereits 9-fach erhöht ist [165]. Daher hat der Nachweis erhebliche Folgen für die weitere Behandlung. Erschwert wird der Nachweis von IEN/Dysplasien durch die hohe Untersuchervariabilität der Pathologen mit einem $\mathrm{k}$-Wert von 0,4 [571], wobei die Variabilität bei niedrig gradigen IEN/Dysplasien und unklaren Veränderungen besonders hoch ist. Wegen der erheblichen therapeutischen Konsequenzen soll daher mindestens eine unabhängige pathologische Zweitbeurteilung erfolgen.

Da es für den Pathologen schwierig sein kann, in entzündlichen Arealen eine IEN/Dysplasie sicher zu beurteilen, kann es bei einer vom Pathologen als fragliche IEN/Dysplasie eingestuften Läsion Sinn machen, innerhalb von 3 Monaten nach einer Intensivierung der antiinflammatorischen Therapie eine endoskopische Kontrolle durchzuführen. 


\section{Empfehlung 8.18*}

Bei dem Nachweis einer eindeutigen, durch einen externen Pathologen bestätigten Kolitis-assoziierten hochgradigen IEN/Dysplasie (C) oder eines Adenokarzinoms (B) soll eine Proktokolektomie erfolgen.

[Evidenzgrad: s. Empfehlungstext, Konsensusstärke: starker Konsens, Empfehlungsstärke: $\uparrow \uparrow$ ]

\section{Empfehlung 8.19}

Bei dem Nachweis einer eindeutigen, durch einen externen Referenzpathologen bestätigten, niedrig gradigen IEN/Dysplasie in flacher Mukosa soll dem Patienten nach Aufklärung über das Malignitätsrisiko entweder eine Proktokolektomie (relative Operationsindikation) oder eine endoskopisch-bioptisch Kontrolle innerhalb von 3 Monaten mit anschließend engmaschiger Überwachung angeboten werden.

[Evidenzgrad: B., Konsensusstärke: Konsens, Empfehlungsstärke: $\uparrow \uparrow]$

\section{Empfehlung $\mathbf{8 . 2 0}$}

Scharf begrenzte, erhabene Läsionen mit intraepithelialen Neoplasien, die vom Pathologen als „adenoma-like mass“ (ALM) klassifiziert sind (siehe AG 3), sollen möglichst endoskopisch oder sonst operativ komplett reseziert werden, sofern sich in gezielt aus der Umgebung entnommenen Biopsien und im Restkolon keine IEN zeigen.

[Evidenzgrad: B, Konsensusstärke: starker Konsens, Empfehlungsstärke: $\uparrow \uparrow$ ]

\section{Erläuterung}

Kolonkarzinome sind bei Patienten mit CU und Nachweis von IEN gehäuft. Grundsätzlich ist zwischen flachen und erhabenen Läsionen zu unterscheiden.

Befindet sich das Kolon nicht in einem weitgehend entzündungsfreien Zustand, können entzündliche Veränderungen Ähnlichkeiten zu IEN haben. Diese werden dann i.d.R. als fragliche IEN bezeichnet und eine Intensivierung der antiinflammatorischen Therapie mit kurzfristiger Kontrolle erscheint sinnvoll. Zeigt sich dagegen eine flache, hochgradige IEN liegt das Risiko, dass gleichzeitig ein unerkanntes Karzinom besteht, zwischen 42 - $45 \%$ [172, 557].

Aus flachen, niedrig gradigen IEN können einerseits hochgradige IEN oder Karzinome hervorgehen, andererseits können niedriggradige IEN ein Indikator für synchrone Karzinome sein. Eine Metaanalyse errechnete ein 9-fach erhöhtes Risiko für die Entwicklung eines Karzinoms, wenn eine niedrig gradige IEN nachgewiesen wird. Andererseits zeigen einzelne Studien, dass das Risiko für die Entwicklung eines Colitis-assoziierten kolorektalen Karzinoms bei Patienten mit niedrig gradigen IEN nicht oder nicht signifikant erhöht ist $[165,167,168]$. Eine populationsbasierte Studie konnte auch keinen vermehrten Übergang zu Karzinomen finden [171]. Die Datenlage ist somit bei den niedrig gradigen IEN kontrovers und eine engmaschige Überwachung mit bioptischer Kontrolle nach 3 Monaten wird als Alternative zur Kolektomie für vertretbar gehalten.

Erhabene Läsionen mit IEN wurden ursprünglich als Dysplasieassoziierte Läsionen oder Massen (DALM) klassifiziert [173]. Das Risiko eines Kolonkarzinoms beim Vorliegen einer DALM wurde als sehr hoch eingeschätzt [172]. Die Einordnung als DALM ist aber nicht ganz einfach und wurde uneinheitlich vorgenommen. Solche Läsionen können auch sporadischen Adenomen ähneln (ALM) und dann endoskopisch reseziert werden (bez. der histologischen Unterscheidung wird auf das Kapitel 3 Histopathologische Diagnostik 3.10 verwiesen). Wenn die Resektion histologisch komplett ist, in der Umgebung keine IEN $\mathrm{zu}$ finden sind und wenn sich im Restkolon keine IEN findet, kann auf eine Kolektomie verzichtet werden. Allerdings sollten diese Patienten kurzfristig kontrolliert werden, weil diese Patienten zur Entwicklung erhabener Läsionen neigen [175, 177, 572].

\section{Chemoprävention}

\section{Empfehlung 8.21*}

Zur Prophylaxe des Colitis-assoziierten Kolonkarzinoms können 5-ASA-haltige Präparate eingesetzt werden.

[Evidenzgrad: B

Konsensustärke: starker Konsens, Empfehlungsstärke: $\uparrow$ ]

\section{Empfehlung 8.22*}

Beim zusätzlichen Nachweis einer PSC kann zur Prophylaxe eines Colitis-assoziierten Kolonkarzinoms Ursodesoxycholsäure eingesetzt werden.

[Evidenzgrad: A

Konsensustärke: starker Konsens, Empfehlungsstärke: $\uparrow$ ]

\section{Erläuterung}

In mehreren Kohorten- sowie Fall-Kontrollstudien konnte gezeigt werden, dass die Einnahme von sowohl Sulfasalazin, als auch 5-ASA mit einem verminderten kolorektalen Karzinomrisiko bei Patienten mit Colitis ulcerosa assoziiert ist [556, 573]. Die Einnahme von 5-ASA-haltigen Präparaten ersetzt jedoch nicht die Notwendigkeit einer regelmäßigen Überwachungskoloskopie.

In einer Follow-Up-Studie einer prospektiven, placebokontrollierten Studie (PSC-UDCA-Studie) konnte gezeigt werden, dass Ursodesoxycholsäure das Kolonkarzinomrisiko bei Patienten mit PSC und CU um 74\% vermindert [574].

Eine Querschnittsuntersuchung aus einer prospektiven Überwachungsstudie hatte zuvor eine deutliche Risikoreduktion gezeigt [575]. Einschränkend ist zu erwähnen, dass eine prospektiv-randomisierte Studie aus dem Jahr 2009 insgesamt eine leicht erhöhte Mortalität bei Patienten mit PSC unter einer Ursodesoxycholsäure-Hochdosistherapie $(28-30 \mathrm{mg} / \mathrm{kg} / \mathrm{d})$ gefunden hat [576]. Vor diesem Hintergrund wird eine Behandlung mit $13-20 \mathrm{mg} / \mathrm{kg}$ KG pro Tag empfohlen.

Immer wieder wird auch die Rolle von Azathioprin/6-Mercaptopurin in der Chemoprävention, einerseits über einen substanzeigenen Effekt und andererseits evtl. auch indirekt über die Unterdrückung der Entzündung, diskutiert [555, 577]. In der Diskussion ist auch, ob neben Azathioprin die zusätzliche Gabe von 5-ASA einen chemopräventiven Effekt haben könnte. Ein systemisches Review aus 2009 zu diesem Thema kommt zu dem Schluss, dass keine vorliegende Studie diese Frage sicher direkt beantworten kann [259]. 


\section{Immunsuppression und Karzinomentstehung}

\section{Empfehlung 8.23}

Patienten unter Therapie mit mindestens 2 Immunsuppressiva sollen regelmäßig dermatologisch untersucht werden und zu konsequentem Sonnenschutz aufgefordert werden.

[Evidenzgrad: $\mathrm{C}$

Konsensustärke: Konsens, Empfehlungsstärke: $\uparrow \uparrow$ ]

\section{Erläuterung}

Aus der Transplantationsliteratur ist bekannt, dass immunsupprimierte Patienten deutlich gehäuft Hautneoplasien, insbesondere Plattenepithelkarzinome, entwickeln [38]. In erster Linie werden hierfür Calcineurininhibitoren und Purinantagonisten verantwortlich gemacht. Für Azathioprin konnte gezeigt werden, dass eine deutlich erhöhte Sensitivität gegenüber UVA-Strahlen besteht $[578,579]$. Es wird daher für transplantierte Patienten, insbesondere wenn zwei Immunsuppressiva gegeben werden, empfohlen, vor Einsatz dieser Medikamente und dann in der Folgezeit jährlich eine dermatologische Kontrolluntersuchung durchzuführen. Es existieren nur wenige Studien über Patienten mit chronisch entzündlichen Darmerkrankungen. Ein Bericht bei nicht transplantierten Patienten, worunter auch Patienten mit Colitis ulcerosa waren, zeigte unter Azathioprin ein 5-fach erhöhtes Risiko für Plattenepithelkarzinome [580]. Insgesamt ist jedoch die Empfehlung v.a. aus der Transplantationsliteratur übertragen (indirekte Anwendbarkeit).

\section{Kapitel 9: Extraintestinale Manifestationen \\ $\nabla$}

Extraintestinale Manifestationen sind Erkrankungen bei Patienten mit Colitis ulcerosa außerhalb des GI-Traktes, bei denen aufgrund der klinischen Assoziation oder pathogenetischer Mechanismen ein Zusammenhang mit der entzündlichen Darmerkrankung besteht oder vermutet werden muss. Hiervon werden extraintestinale Begleiterkrankungen (Gallensteine, Nierensteine) und nicht krankheitsspezifische Komplikationen (Osteoporose, thromboembolische Komplikationen) abgegrenzt.

\section{Gelenkmanifestationen}

\section{Statement 9.1}

Die Gelenkbeteiligung stellt die häufigste extraintestinale Manifestation bei der Colitis ulcerosa dar. Es wird zwischen dem Befall des Achsenskeletts und dem peripheren Gelenkbefall, der meist in Form von Arthralgien imponiert, unterschieden.

[Evidenzgrad: D, Konsensusstärke: starker Konsens]

\section{Erläuterung}

Arthritiden bei Patienten mit CU werden im Rahmen der HLAB27-assoziierten seronegativen Spondylarthropathien (SpA) klassifiziert. Die Klassifikationskriterien der europäischen Spondyloarthritis-Studiengruppe sind bei einem CED-Patienten erfüllt, sofern a) ein früherer oder gegenwärtiger entzündlicher Wirbelsäulenschmerz von mindestens 3 Monaten Dauer oder b) eine asymmetrisch oder vorwiegend an den unteren Extremitäten bestehende Synovitis vorliegt [581]. Orchard et al. haben darüber hinaus bei Patienten mit CED eine weitere Unterteilung der peripheren Arthritiden vorgeschlagen [582]. Der schubassoziierte, meist selbstlimierte Typ I ist pauciartikulär (<5 Gelenke) und betrifft meist die großen Gelenke, der Typ II verläuft als chronisch bilateral symmetrische Polyarthropathie ( $\geq 5$ Gelenke) unabhängig von der CED-Aktivität. Bei Patienten mit CED wird die Prävalenz der SpA in spezialisierten Zentren mit 10-32\% angegeben [583-588]. Sie ist somit bei einer geschätzten Häufigkeit in der deutschen Allgemeinbevölkerung von 1,9\% deutlich erhöht [589]. Gelenkbeschwerden gehen mit einem erhöhten Risiko für die Entwicklung einer Pouchitis einher und treten auch nach Kolektomie auf [529, 590].

\section{Empfehlung 9.2}

Die Diagnose der Gelenkbeteiligung soll durch Anamnese, klinische Untersuchung und bei axialem Befall auch bildgebend entsprechend rheumatologischer Kriterien erfolgen.

[Evidenzgrad: C, Konsensusstärke: starker Konsens, Empfehlungsstärke: $\uparrow \uparrow$ ]

\section{Erläuterung}

Der Befall des Achsenskeletts und der peripheren Gelenke im Rahmen der CU kann nicht von anderen Formen seronegativer Spondylarthropathien unterschieden werden [582, 585, 591]. Anamnestisch und durch Funktionsuntersuchungen sollte überprüft werden, ob entweder Arthralgien (nur Gelenkschmerzen), periphere Synovialitiden im Sinne von peripheren Arthritiden (s.o., Typ I oder Typ II) und/oder ein entzündlicher Rückenschmerz als Kardinalsymptom bei axialem Befall vorliegt. Die periphere Gelenkbeteiligung weist in der Regel keine radiologischen Veränderungen auf, die entzündlichen Veränderungen des Achsenskeletts werden am sensitivsten durch die Kernspintomografie dargestellt.

Folgende Differenzialdiagnosen sollten bei muskuloskelettalen Beschwerden bedacht werden: Arthrose, septische Arthritis, Spondylodiscitis, Osteoporose, hypertrophe Osteoarthropathie, avaskuläre Osteonekrose, co-incidente rheumatoide Arthritis, Pyrophosphatarthropathie, Gicht, gelenknahes Erythema nodosum oder Therapienebenwirkungen (steroidinduzierter Pseudorheumatismus nach Absetzen einer Steroid-Langzeittherapie und die Mesalazin- oder Azathioprin-induzierte Arthropathie).

\section{Empfehlung 9.3}

Bei schubassoziierter Gelenkbeteiligung soll die Therapie im Rahmen der Behandlung der Grunderkrankung erfolgen.

[Evidenzgrad: A, Konsensusstärke: Konsens, Empfehlungsstärke: $\uparrow \uparrow]$

\section{Empfehlung 9.4}

Bei der akuten Arthritis können begleitende symptomatische Maßnahmen wie Entlastung und Ruhigstellung akut betroffener Gelenke erfolgen.

[Evidenzgrad: D, Konsensusstärke: starker Konsens, Empfehlungsstärke: $\uparrow$ ] 


\section{Empfehlung 9.5}

a) Bei chronischen Arthralgien kann eine Physiotherapie erfolgen.

b) Eine analgetische Therapie nach allgemeinen Empfehlungen zur Schmerztherapie soll durchgeführt werden.

c) NSAR sollten eher nicht eingesetzt werden.

[Evidenzgrad: A) D; B) D; C) B, Konsensusstärke: Konsens, Empfehlungsstärke: A) $\uparrow$; B) $\uparrow \uparrow ; C) \downarrow$ ]

\section{Empfehlung 9.6}

a) Bei peripheren Arthritiden soll primär Sulfasalazin eingesetzt werden.

b) Bei schweren peripheren Arthritiden kann Methotrexat eingesetzt werden.

[Evidenzgrad: A) B; B) B, Konsensusstärke: Konsens, Empfehlungsstärke: $A) \uparrow \uparrow B) \uparrow]$

\section{Empfehlung 9.7}

Schwere, therapierefraktäre Polyarthritiden und die schwere therapierefraktäre Spondylarthropathie (Spondylitis ankylosans) können mit anti-TNF- $\alpha$-Antikörpern behandelt werden.

[Evidenzgrad: B, Konsensusstärke: starker Konsens, Empfehlungsstärke: $\uparrow$ ]

\section{Erläuterung}

Für die Behandlung von Gelenkbeschwerden bei Patienten mit CU existieren nur sehr wenige Therapiestudien. Die angegebenen Empfehlungen sind daher überwiegend der klinischen Erfahrung sowie der rheumatologischen Literatur entlehnt (indirekte Anwendbarkeit für Sulfasalazin und anti-TNF- $\alpha$-Antikörper). Sulfasalazin, Steroide, Methotrexat, Azathioprin und antiTNF- $\alpha$-Antikörper haben antientzündliche Effekte auf chronisch entzündliche Erkrankungen des Darmes und der Gelenke [592 -594]. Bei unzureichender Wirkung der systemischen Therapien stellt die intraartikuläre Injektion von Glukokortikoiden eine weitere Therapieoption dar. Die Physiotherapie ist Basistherapie bei der Spondylitis ankylosans. Der Einsatz von NSAR bei Colitis ulcerosa wird in der Literatur kontrovers diskutiert, da hierdurch möglicherweise Schübe induziert werden können [595-597]. Ob die COX-2-Hemmer in diesem Zusammenhang in der breiten Anwendung sicherer sind, bleibt trotz zweier RCT, die bei COX-2-Hemmer Therapie keine gehäufte Exazerbation der Colitis ulcerosa fanden, fraglich [18, 598, 599].

Die Therapieempfehlung von anti-TNF- $\alpha$-Antikörpern (Infliximab und Adalimumab) bei schwerer therapierefraktärer Polyarthritis und Spondylarthropathie (Spondylitis ankylosans) beruht zumeist auf deren Wirksamkeit und Zulassung bei der rheumatoiden Arthritis und der Spondylitis ankylosans [600-605].

\section{Manifestation an der Leber und an den Gallenwegen}

\section{Statement 9.8}

Klinisch ist die primär sklerosierende Cholangitis (PSC) die bedeutendste hepatobiliäre Erkrankung, die mit einer Colitis ulcerosa assoziiert sein kann. Die Häufigkeit der PSC bei Colitis ulcerosa liegt bei $2-10 \%$ der Patienten. Die Autoimmunhepatitis ist eine seltene Begleiterkrankung der Colitis ulcerosa und kann in Form eines Überlappungssyndroms mit einer PSC auftreten.

[Evidenzgrad: D, Konsensusstärke: starker Konsens]

\section{Empfehlung 9.9}

Bei Verdacht auf eine PSC soll eine MRCP als primäre Diagnostik erfolgen. Bei unklaren Fällen oder fortbestehendem klinischem Verdacht soll eine ERC ergänzend durchgeführt werden.

[Evidenzgrad: C, Konsensusstärke: Konsens, Empfehlungsstärke: $\uparrow \uparrow]$

\section{Erläuterung}

Die ERC und die MRCP sind in der Wertigkeit für die Diagnosestellung gleichwertig [606, 607]. Aufgrund der geringeren Invasivität wird die MRCP häufig als Erstlinienuntersuchung durchgeführt. Falls diese nicht eindeutig ausfällt und/oder ein klinischer Verdacht auf eine PSC fortbesteht, ist eine nachfolgende ERC indiziert. Die Leberbiopsie mit Histologie dient der Stadieneinteilung der PSC und dem Nachweis der „Small duct disease“. Die PSC ist ein wichtiger Risikofaktor zur Entstehung von Cholangiokarzinomen [608]. Sinnvolle Strategien zum Screening oder zur Früherkennung von Cholangiokarzinomen existieren nicht, die amerikanische Leitlinie zur PSC empfiehlt jedoch bei diesen Patienten jährlich eine Ultraschalluntersuchung der Gallenblase mit dem Ziel der Früherkennung von Gallenblasenkarzinome [609].

\section{Empfehlung 9.10}

Ab Diagnosestellung einer PSC sollte eine Dauertherapie mit Ursodeoxycholsäure durchgeführt werden.

[Evidenzgrad: B, Konsensusstärke: starker Konsens, Empfehlungsstärke: $\uparrow$ ]

\section{Empfehlung 9.11}

Zusätzlich soll eine endoskopische Therapie von funktionell wirksamen und erreichbaren Stenosen durchgeführt werden.

[Evidenzgrad: D, Konsensusstärke: starker Konsens, Empfehlungsstärke: $\uparrow \uparrow$ ]

\section{Empfehlung 9.12}

In fortgeschrittenen Stadien der PSC soll in einem Transplantationszentrum die Möglichkeit einer Lebertransplantation geprüft werden.

[Evidenzgrad: A, Konsensusstärke: starker Konsens, Empfehlungsstärke: $\uparrow \uparrow$ ]

\section{Erläuterung}

Ursodeoxycholsäure in einer Dosierung von $15-20 \mathrm{mg} / \mathrm{kg}$ führt zur Besserung der Serumtransaminasen und Cholestaseparameter und Prognosefaktoren wie Mayo Risk Score, Histologie und Cholangiografie [610]. In keiner der bisher durchgeführten Studien konnte jedoch ein statistisch signifikanter Einfluss auf das Überleben nachgewiesen werden. Eine placebokontrollierte Hochdosis-Therapie-Studie mit $28-30 \mathrm{mg} / \mathrm{kg}$ Ursodeoxycholsäure führte in einer neuen Studie bei Patienten mit fortgeschrittener PSC sogar zu schlechteren Ergebnissen als in der Kontrollgruppe bez. der definierten Endpunkte wie Lebertransplantation oder Entwicklung von Oesophagusvarizen [576]. Deshalb sollte die Hochdosisbehandlung mit 28-30 mg/ $\mathrm{kg}$ Ursodeoxycholsäure bei Patienten im Endstadium der PSC vermieden werden. Endoskopische Dilatation von größeren 
Gangstenosen verbessert signifikant den Verlauf und das Überleben der Patienten [611, 612].

\section{Hautmanifestationen bei Colitis ulcerosa}

\section{Statement 9.13}

Typische Hautmanifestationen sind Erythema nodosum (14 - 19\% der Patienten) und Pyoderma gangraenosum (1 - $2 \%$ der Patienten). Überwiegend treten die Effloreszensen im akuten Schub der Erkrankung auf. Die Diagnose erfolgt klinisch. Serologische Marker existieren nicht.

[Evidenzgrad: D, Konsensusstärke: Konsens]

\section{Empfehlung 9.14}

a) Bei Erythema nodosum und Pyoderma gangraenosum sollte eine hoch dosierte Steroidtherapie erfolgen und die Colitis ulcerosa in Remission gebracht werden.

b) In therapieresistenten Fällen sollte eine immunmodulatorische Therapie durchgeführt werden.

c) Eine chirurgische Intervention soll nicht durchgeführt werden. [Evidenzgrad: D, Konsensusstärke: Konsens, Empfehlungsstärke: A) $\uparrow$, B) $\uparrow$, C) $\downarrow \downarrow]$

\section{Erläuterung}

Die Biopsie des Pyoderma gangraenosum kann zu einer Exazerbation der Läsion führen und ist daher nicht sinnvoll [613]. Über die Behandlung von Hautmanifestationen liegen weder kontrollierte noch Fallkontrollstudien vor. Umfangreiche Fallserien beschreiben den erfolgreichen Einsatz von hoch dosierten systemischen Steroiden insbesondere beim Erythema nodosum. Fallberichte und Fallserien berichten für das Pyoderma gangraenosum über den erfolgreichen Einsatz einer Reihe von Immunsuppressiva [614, 615].

\section{Augenmanifestationen bei Colitis ulcerosa}

\section{Statement 9.15}

Als extraintestinale Manifestationen treten die anteriore Uveitis (Iritis/Iridocyclitis), die Skleritis und die Episcleritis bei 1,4-22,9\% der Patienten mit Colitis ulcerosa auf.

[Evidenzgrad: D, Konsensusstärke: Konsens]

\section{Empfehlung 9.16}

Bei Verdacht auf eine okuläre Manifestation bei Colitis ulcerosa soll eine fachärztliche, ophthalmologische Untersuchung durchgeführt werden. Die Episkleritis soll primär nicht systemisch therapiert werden, sondern die topische Gabe von Kortikosteroiden erfolgen. Die Skleritis soll mit systemischen Kortikosteroiden, ggf. auch mit Immunsuppressiva behandelt werden. Die Uveitis soll je nach Lokalisation mit topischen oder systemischen Kortikosteroiden behandelt werden.

[Evidenzgrad: D, Konsensusstärke: starker Konsens, Empfehlungsstärke: $\uparrow \uparrow$ ]

\section{Empfehlung 9.17}

Eine immunsuppressive Therapie kann in resistenten Fällen einer okulären Manifestation durchgeführt werden (C). Zusätzlich kann eine Pupillenerweiterung zur Therapie des Spasmus sowie zur Prophylaxe von Synechien durchgeführt werden.

[Evidenzgrad: D, Konsensusstärke: starker Konsens, Empfehlungsstärke: $\uparrow]$

\section{Erläuterung}

Die Datenlage zur Prävalenz ist uneinheitlich $[583,616]$. Die Therapieempfehlungen stützen sich lediglich auf Fallberichte und Fallsammlungen [617]. Eine immunsuppressive Therapie mit Azathioprin oder Methotrexat scheint in komplizierten Fällen hilfreich zu sein [618]. Bei therapierefraktärer Uveitis wurden in Fallberichten erfolgreiche Therapien mit Cyclosporin A [619] oder Infliximab [620] beschrieben.

\section{Osteoporose}

\section{Statement 9.18}

Die Häufigkeit einer Osteoporose bei Patienten mit Colitis ulcerosa schwankt zwischen 7 und $18 \%$, eine Osteopenie liegt bei 34 $67 \%$ aller Patienten vor. Das Risiko für Wirbelfrakturen oder Hüftfrakturen ist bei Patienten mit Colitis ulcerosa 1,4-fach gegenüber der Normalbevölkerung erhöht.

[Evidenzgrad: C, Konsensusstärke: starker Konsens]

\section{Empfehlung 9.19}

Eine Knochendichtemessung (DXA) zur Diagnose einer Osteoporose soll bei Patienten durchgeführt werden, die mit systemischen Steroiden längerfristig behandelt wurden und/oder eine chronische Entzündungsaktivität aufweisen.

[Evidenzgrad: C, Konsensusstärke: starker Konsens, Empfehlungsstärke: $\uparrow \uparrow$ ]

\section{Erläuterung}

In den gängigen Scores und Startifizierungstabellen geht die Diagnose einer chronisch entzündlichen Darmerkrankung nicht in die Abschätzung des individuellen Osteoporoserisikos ein. Auch für Patienten mit chronisch entzündlichen Darmerkrankungen ist jedoch ein Zusammenhang zwischen Knochendichte und Frakturinzidenz nachgewiesen [621]. Kontrolluntersuchungen der Knochendichte unter Therapie sind frühestens nach einem Jahr indiziert. Andere diagnostische Verfahren sind aufgrund höherer Strahlenbelastung und geringerer Verfügbarkeit (quantitative CT) bzw. fehlender Standardisierung (Osteosonografie) derzeit nicht sinnvoll. In der Diagnostik einer Osteoporose spielen knochenspezifische Laborparameter keine Rolle. Validierte genetische Marker existieren nicht [622].

\section{Empfehlung 9.20}

Im Rahmen einer systemischen Steroidmedikation soll eine Substitution mit Kalzium und Vitamin D erfolgen. Die Therapie der Osteopenie und der Osteoporose soll durch die Substitution mit Kalzium und Vitamin D erfolgen (C). Bei Patienten mit Frakturen soll eine Aminobisphosphonattherapie erfolgen (A).

[Evidenzgrad: siehe Empfehlungstext, Konsensusstärke: starker Konsens, Empfehlungsstärke: $\uparrow \uparrow$ ] 


\section{Erläuterung}

Der Therapieeffekt einer Substitution mit Kalzium und Vitamin D auf die Knochendichte bei Colitis ulcerosa wurde bisher nur in kleinen Studien überprüft. Für die Therapie von Patienten mit steroidinduzierter Osteoporose existiert eine sehr gute konfirmatorische Datenlage [622].

Bisher gibt es keine Daten zur Effektivität einer Therapie mit Aminobisphosphonaten bei Patienten mit Colitis ulcerosa. Auch bei Patienten mit Morbus Crohn ist nicht nachgewiesen, dass die therapeutische Anhebung der Knochendichte die Frakturrate im Sinne einer Primärprävention reduziert. Bei Patienten mit nachgewiesenen Frakturen wird analog der allgemeinen osteologischen Leitlinien bei steroidinduzierter Osteoporose behandelt [622].

Seltene extraintestinale Manifestationen und extraintestinale Begleiterkrankungen

\section{Statement 9.21}

Neben den oben behandelten gut definierten extraintestinalen Manifestationen bei Colitis ulcerosa gibt es zusätzlich noch weniger gut definierte kasuistisch belegte extraintestinale Manifestationen im Bereich der Lunge, des Herzen, des Pankreas, der Nieren sowie neurologischer Manifestationen. Zusätzlich treten im Sinne von extraintestinalen Begleiterkrankungen im Vergleich zur Normalbevölkerung häufiger Gallensteine, Nierensteine sowie thromboembolische Komplikationen auf.

[Evidenzgrad: D, Konsensusstärke: starker Konsens]

\section{Erläuterung}

$\mathrm{Zu}$ dieser Thematik wird aufgrund der niedrigen Prävalenz und der uneinheitlichen Datenlage bei Patienten mit Colitis ulcerosa auf den entsprechenden Text in den Leitlinien Morbus Crohn - Extraintestinale Manifestationen verwiesen.

\section{Kapitel 10: Psychosomatik, Komplementär- und Alternativmedizin}

\section{Psychosomatik}

\section{Einleitung}

Der Einfluss psychosozialer Faktoren auf die Entstehung der Colitis ulcerosa ist gering. Es wird ein mäßiger Einfluss psychosozialer Faktoren auf den Verlauf der Erkrankung angenommen. Ein erweitertes biopsychosoziales Modell [623, 624] berücksichtigt die komplexen biologischen und psychosozialen Interaktionen mit Auswirkungen auf die Lebensqualität und den Krankheitsverlauf bei Patienten mit Colitis ulcerosa.

\section{Statement 10.1}

Belastende Lebensereignisse, psychologischer Stress und psychische Störungen sind nicht ursächlich für die Entstehung der Colitis ulcerosa.

[Evidenzgrad: B, Konsensusstärke: starker Konsens]

\section{Erläuterung}

Eine systematische Übersichtsarbeit fand keine eindeutigen Hinweise, dass prämorbide seelische Störungen einen Risikofaktor für die Entstehung einer Colitis ulcerosa darstellen [625].

\section{Statement 10.2}

Subjektive Stressbelastung und affektive Störungen können einen negativen Einfluss auf den Verlauf der Colitis ulcerosa haben.

[Evidenzgrad: B, Konsensusstärke: starker Konsens]

\section{Erläuterung}

Systematische Literaturübersichten erbrachten widersprüichliche Assoziationen zwischen depressiven Störungen, belastenden Lebensereignissen bzw. subjektiv empfundenem Stress und dem Verlauf bei chronisch-entzündlichen Darmerkrankungen [626, 627]. In 13 von 18 prospektiven Studien (10-155 Patienten) zeigte sich ein Zusammenhang zwischen Stress und „adverse outcomes“. Die Qualität der Evidenz wurde teilweise durch methodische Mängel der Studien reduziert [626]. Die Betroffenen erachten psychischen Stress als rezidivauslösend $[628,629]$.

\section{Statement 10.3}

Eine hohe Krankheitsaktivität kann mit vermehrter psychischer Symptombelastung einhergehen.

[Evidenzgrad: B, Konsensusstärke: starker Konsens]

\section{Erläuterung}

Zwei systematische Übersichtsarbeiten fanden keine einheitlichen Ergebnisse, ob psychische Störungen häufiger bei Patienten mit Colitis ulcerosa und Morbus Crohn auftreten als bei Patienten mit anderen chronischen körperlichen Erkrankungen. Ängstlichkeit und depressive Stimmungslage sind mit der Krankheitsschwere korreliert. Patienten mit Reizdarm-Symptomatik (RDS bzw. IBS) in Remission haben eine erhöhte Rate von psychischen Störungen im Vergleich zu Kontrollgruppen $[625,630]$. Die negativen psychosozialen Konsequenzen der Erkrankung nehmen mit der Schwere der Erkrankung zu. Die Lebensqualität wird durch die Krankheitsaktivität beeinträchtigt [631-638].

\section{Statement 10.4}

Psychische Störungen können einen negativen Einfluss auf den Krankheitsverlauf und die Lebensqualität haben.

[Evidenzgrad: B, Konsensusstärke: starker Konsens]

\section{Erläuterung}

Psychische Störungen wie Angst und Depression beeinträchtigen auch unabhängig von der Schwere der Colitis ulcerosa die Lebensqualität [632, 635, 639-641]. Depressive Copingstrategien beeinflussen negativ die zukünftige Lebensqualität [642].

\section{Empfehlung 10.5}

Patienten mit anhaltenden Bauchschmerzen oder Durchfällen, welche nicht durch die Krankheitsaktivität bzw. Krankheitskomplikationen erklärt werden können, sollten auf das Vorliegen eines Reizdarmsyndroms (RDS) oder einer depressiven Störung untersucht werden. Bei Vorliegen eines RDS bzw. einer depressiven Störung sollten die in Leitlinien empfohlenen Therapieprinzipien angewendet werden.

[Evidenzgrad: B, Konsensusstärke: Konsens, Empfehlungsstärke: $\uparrow$ ] 


\section{Erläuterung}

Eine Subgruppe von Patienten gibt anhaltende Bauchschmerzen und/oder Durchfälle sowie reduzierte Lebensqualität bei fehlender oder geringer laborchemischer und endoskopischer/histologischer Aktivität an. In diesem Fall ist zu überprüfen, ob die Kriterien eines Reizdarmsyndroms erfüllt sind. Die Prävalenzraten eines Reizdarmsyndroms nach den Rom-Kriterien bei klinischen Stichproben liegen zwischen 9-46\% [643-646]. Colitis-ulcerosa-Patienten mit Reizdarmsyndrom (RDS) weisen eine vermehrte psychische Symptombelastung mit Angst/Depressivität und eine geringere Lebensqualität auf als Patienten ohne RDS bzw. komorbide psychische Störungen. Es liegen keine Therapiestudien zur Behandlung eines RDS bei Colitis ulcerosa vor. Die Behandlung sollte gemäß den Leitlinien von gastroenterologischen Fachgesellschaften medikamentös (Spasmolytika, Antidepressiva) und psychotherapeutisch erfolgen [647].

\section{Empfehlung $10.6^{*}$}

Psychosoziale Faktoren und die krankheitsbezogene Lebensqualität sollen auch unter Berücksichtigung geschlechtsspezifischer Aspekte bei ärztlichen Konsultationen erfragt und in der Therapie berücksichtigt werden.

[Evidenzgrad: B, Konsensusstärke: starker Konsens, Empfehlungsstärke: $\uparrow \uparrow$ ]

\section{Erläuterung}

Zur Messung der Lebensqualität haben sich 2 validierte krankheitsspezifische Fragebogen als sensitiv, reproduzierbar und in klinischen Studien anwendbar erwiesen. So ist für den Inflammatory Bowel Disease Questionnaire (IBDQ) [648] inzwischen eine deutsche Übersetzung validiert [649]. Für den klinischen Alltag bewährt sich die Kurzform SIBDQ [650], von dem die deutschsprachige Version gute Testgütekriterien zeigte [651]. Die Rating Form of Inflammatory Bowel Disease Patient Concerns (RFIPC) [652] misst subjektive Befürchtungen der Patienten.

Alltagstaugliche Screeninginstrumente zur Erfassung des Bedarfs an psychosozialer Behandlung sind der ADAPT (Assessment of the Demand für Additional Psychological Treatment) [640] und der LIPS (Das Lübeck Interview für Psychosoziales Screening) [653]. Zur Erfassung von Depression oder Angst sind wenige Fragen ausreichend [654, 655].

Da Strategien zur sozialen Unterstützung psychologischen Stress vermindern können [656], sollten Gastroenterologen und Ärzte, die Patienten mit chronisch-entzündlichen Darmerkrankungen behandeln, ihre psychosoziale Kompetenz kontinuierlich weiterentwickeln. Dabei ist zu berücksichtigen, dass psychisch belastete Patienten weniger Informationen aufnehmen [657] und Geschlechtsunterschiede in der Einschätzung der Lebensqualität bestehen [633, 658, 659].

Bei einer Befragung von Mitgliedern der Deutschen Morbus Crohn/Colitis ulcerosa Vereinigung (DCCV) zeigte sich eine Einschränkung der Sexualität bei Frauen in Verbindung mit depressiver Verstimmung [660], nicht jedoch bei Männern [660]. In der Untersuchung von Fuller-Thomson et al. [661] dachten in der Gruppe der depressiven Patienten mehr Frauen als Männer an Suizid (50 vs. 31\%). Depressives Coping verbesserte sich nach einer psychotherapeutischen Behandlung nur bei Frauen [662]. In einer postalischen Befragung gaben Frauen häufiger Beeinträchtigungen hinsichtlich Körperbild, Libido und sexueller Aktivität als Männer an [663]. Ängste können zu freiwilliger Kinderlosigkeit führen [663].

\section{Empfehlung 10.7}

Bei der Behandlung von Patienten mit Colitis ulcerosa sollen Kooperationen mit Psychotherapeuten bzw. Psychosomatikern bestehen.

[Evidenzgrad: B, Konsensusstärke: Konsens, Empfehlungsstärke: $\uparrow \uparrow]$

\section{Erläuterung}

Da nahezu ein Drittel aller Erkrankten in tertiären Zentren einen hohen Bedarf an zusätzlicher psychischer Betreuung angibt, ist die Vermittlung einer psychosomatisch-psychotherapeutischen Behandlung in der klinischen Routine wichtig [664]. Die meisten Experten sehen sich in der Lage, ihren Patienten eine Psychotherapie zu empfehlen. Auch wenn Studien dazu fehlen, wird diese Auffassung von Teilnehmern deutscher und europäischer Konsensusgruppen geteilt [10, 665, 666].

\section{Empfehlung 10.8}

Die behandelnden Ärzte sollen auf die Selbsthilfe hinweisen und die Patienten über ihre Krankheit informieren.

[Evidenzgrad: D, Konsensusstärke: Konsens, Empfehlungsstärke: $\uparrow \uparrow]$

\section{Erläuterung}

Krankheitsinformationen sollen dem Patienten empathisch in einem persönlichen, emotional stützenden Gespräch vermittelt werden. Schlecht informierte Patienten entwickeln stärkere Befürchtungen [667] und psychosoziale Faktoren wie schlechte Lebensqualität, subjektive Krankheitsbelastung und Depression korrelieren mit dem Inanspruchnahmeverhalten medizinischer Einrichtungen [639, 664]. Patienten beklagen häufig unzureichende Informationen, Mängel bei der Betreuung ihrer Angehörigen und der medizinischen Versorgung [668].

Patientenzentrierte Konsultationen in Verbindung mit schriftlichen Anleitungen zum Selbstmanagement verbessern die Krankheitskontrolle [669] und die Compliance [670]. Schriftliche Informationen alleine erscheinen jedoch nicht hilfreich, sondern können zur Verschlechterung der krankheitsspezifischen Lebensqualität beitragen [671]. Eine webbasierte Schulung steigerte zwar das Wissen und die Möglichkeit der Selbsttherapie, scheint aber nicht die Lebensqualität, Angst oder Depressivität zu verändern [672].

In Langzeituntersuchungen konnte bisher kein Nachweis für einen positiven Einfluss von Schulungsprogrammen auf Krankheitsverlauf, Lebensqualität oder psychisches Befinden erbracht werden [673-676], obwohl in den meisten Studien die Schulung von den Betroffenen sehr positiv bewertet wurde.

\section{Empfehlung 10.9}

Bei Patienten mit Colitis ulcerosa und psychischen Störungen soll eine Psychotherapie durchgeführt werden.

[Evidenzgrad: B, Konsensusstärke: Konsens, Empfehlungsstärke: $\uparrow \uparrow]$ 


\section{Erläuterung}

Die Diagnose Colitis ulcerosa an sich stellt keine Indikation für eine Psychotherapie dar. Psychotherapiestudien bei Patienten ohne psychische Störungen zeigten geringe oder keine Effekte auf den Krankheitsverlauf [677-679]. In der ärztlichen Routine ist vorwiegend auf Angst, mangelnde soziale Unterstützung und junges Erkrankungsalter als wesentliche Faktoren für den subjektiven Bedarf an zusätzlicher psychischer Betreuung zu achten [664]. Psychotherapie und Entspannungsmethoden haben einen positiven Einfluss durch die Verbesserung der psychischen Befindlichkeit, der Lebensqualität und der Bewältigungsstrategien sowie die Verminderung der psychischen Stressbelastung und des Schmerzempfindens [662, 680-684]. Es gibt keine evidenzbasierten Daten für die Wahl einer bestimmten psychotherapeutischen Methode. Entspannungsübungen werden als sinnvoll erachtet, da sie leicht zu erlernen und anzuwenden sind. Eine experimentelle, kontrollierte Studie zum Effekt einer Hypnosebehandlung zeigte, dass bei aktiver Colitis ulcerosa sowohl systemische als auch mukosale Entzündungsparameter durch Hypnose vermindert werden können [685]. Nach Expertenmeinung ist es vorteilhaft, wenn die Psychotherapeuten Erfahrung in der Behandlung von Patienten mit chronisch-entzündlichen Erkrankungen besitzen und eng mit den behandelnden Gastroenterologen zusammenarbeiten.

Auch wenn bisher keine evidenzbasierten Studien zum Einsatz von Psychopharmaka bei der Colitis ulcerosa vorliegen [630, 686], sind die meisten Experten der Auffassung, dass es klinische Situationen gibt, die den Einsatz von Antidepressiva erfordern.

\section{Empfehlung 10.10}

Kindern und Jugendlichen und ihren Familien soll eine psychosoziale Unterstützung angeboten werden.

[Evidenzgrad: A, Konsensusstärke: Konsens, Empfehlungsstärke: $\uparrow \uparrow]$

\section{Erläuterung}

Kinder und Jugendliche mit chronisch-entzündlichen Darmerkrankungen haben ein höheres Risiko für die Entwicklung von Verhaltensauffälligkeiten, sozialen Dysfunktionen, niedrigem Selbstwertgefühl oder psychischen Störungen, wie Depressionen (bis zu 25\% der Patienten) oder Angst [687-693]. Die Lebensqualität von Adoleszenten mit Colitis ulcerosa und Morbus Crohn ist niedriger als die gesunder Kontrollpersonen [694-697]. Eine Metaanalyse von 19 Studien zur psychischen Anpassung bei 1167 Jugendlichen zeigte häufigere depressive Störungen nicht nur im Vergleich zu gesunden, sondern auch zu anderen chronisch kranken Kindern und Jugendlichen [698]. Die Betroffenen schätzen ihre Bewältigungsstrategien positiver ein als die Eltern [699].

Studien zu psychotherapeutischen Interventionen bei Kindern und Jugendlichen $[684,700]$ ebenso wie die zu sozialer Unterstützung [701] zeigen positive Effekte auf die Lebensqualität und die Depression.

\section{Komplementär- und Alternativmedizin}

\section{Statement 10.11}

Es gibt keine allgemein akzeptierte Definition komplementärer und alternativer Therapieverfahren. Der Begriff komplementärmedizinische Verfahren beinhaltet, dass die angewendeten Behandlungsmethoden additiv, d.h. als Ergänzung zu konventionellen Standardtherapien angewendet werden. Verfahren, welche die konventionellen Standardtherapien ausschließen, werden als alternative Therapieverfahren bezeichnet. Unkonventionelle Therapien sind alle Verfahren, die als nicht anerkannt und/oder wissenschaftlich überprüft gelten. Integrative Medizin verbindet konventionelle und komplementäre Therapie zu einer ,best practice'. [Evidenzgrad: D, Konsensusstärke: starker Konsens]

\section{Erläuterung}

Die Bezeichnung „alternative Therapieverfahren“ spiegelt in der Regel nicht die übliche Anwendungssituation wider, da komplementärmedizinische/naturheilkundliche Therapien keinesfalls ein Selbstverständnis beinhalten, eine Alternative zur konventionellen Therapie darzustellen. Die angloamerikanische Literatur unterscheidet weniger in alternativ und komplementär und verwendet den gemeinsamen Terminus „complementary and alternative medicine“ (CAM), der sich inzwischen international weitgehend durchgesetzt hat. Neuere Literatur spricht bei kombinierter Anwendung von konventioneller und komplementärer Methoden auch von integrativer Medizin.

\section{Empfehlung 10.12}

Alternativtherapien anstatt einer evidenzgesicherten Therapie sollen abgelehnt werden.

[Evidenzgrad: D, Konsensusstärke: starker Konsens, Empfehlungsstärke: $\downarrow \downarrow]$

\section{Erläuterung}

Wenn Alternativtherapien anstatt einer evidenzgesicherten Therapie benutzt werden, ist dieses abzulehnen, da definitionsgemäß keine äquipotente Wirkung nachgewiesen ist (Phytotherapeutika und andere immunmodulierenden Substanzen mit geprüfter Äquipotenz zu einer Standardtherapie sind somit keine alternativen Heilmittel (siehe unten)). Komplementäre Therapien, welche als Ergänzung einer Standardtherapie angewendet werden, sollten in Absprache mit den behandelnden Ärzten erfolgen und können eine Standardtherapie unterstützen.

Das große öffentliche Interesse an Alternativ- und Komplementärmedizin rechtfertigt eine weitere Evaluation dieser Methoden. Das schließt die folgenden Methoden ein: Traditionelle Chinesische Medizin inkl. Akupunktur, Anthroposophische Medizin, Aromatherapie, Ayurvedische Medizin, Homöopathie, immunmodulative Therapien, manuelle Therapien (Osteopathie, Massagen etc.), Mind/Body Medizin, Nahrungsergänzungsmittel, Naturheilkunde, Qi Gong, Reiki.

\section{Empfehlung 10.13}

Die Beurteilung naturheilkundlicher und komplementärmedizinischer Verfahren soll nach Kriterien einer evidenzbasierten Medizin erfolgen.

[Evidenzgrad: D, Konsensusstärke: starker Konsens, Empfehlungsstärke: $\uparrow \uparrow$ ] 


\section{Erläuterung}

Methodik und Fragestellung bestimmen gemäß CONSORT-Konsens die Bewertung einer Evidence-based-Medicine (EBM)Hierarchisierung. Dieses ist bei der Bewertung komplementärmedizinischer Literatur zu berücksichtigen.

Einige komplementäre Therapieverfahren (sog. komplexe individuelle therapeutische Interventionen) beziehen sich auf das salutogenetische Potenzial des Patienten und bedürfen der nicht verblindeten Arzt-Patienten-Beziehung, da korrigierende Interaktionen durch Verblindung zwischen Arzt und Patient nicht möglich sind. Salutogene Therapieansätze sind meist durch Lern- und Regulationsprozesse gekennzeichnet, die durch eine analoge Beziehungsgestaltung des Lehrers zu seinen Schülern gekennzeichnet ist und als dialogisch charakterisiert werden können. Ein Randomised-controlled-trial (RCT)Studiendesign ist daher nicht immer durchführbar, dies ist bei einer EBM-Hierachisierung zu berücksichtigen.

Ferner zeichnen sich komplementär- und alternativmedizinische Verfahren meist durch komplexe Verfahrensweisen aus, die durch einfache Wirknachweise eines Einzelfaktors nicht zu belegen sind, sondern systemisch erfasst werden müssen. Dazu sind Outcomestudien im Kohortenvergleich die geeignete Evaluationsmethode und in ihren Ergebnissen meist dem RCT gleichwertig.

\section{Empfehlung 10.14*}

Patient/-innen sollen über die Anwendung komplementärer Heilmethoden befragt werden. Der behandelnde Arzt soll mit ihnen über ihre Gründe für die Anwendung komplementärmedizinischer Verfahren sprechen.

[Evidenzgrad: D, Konsensusstärke: starker Konsens, Empfehlungsstärke: $\uparrow \uparrow$ ]

\section{Erläuterung}

In zahlreichen Studien wurde beschrieben, dass etwa die Hälfte der Patienten mit CED komplementäre Heilmethoden anwendet (31-68\%) [702-704]. Bei Kindern mit CED ist der Gebrauch von CAM nicht geringer als bei Erwachsenen.

In einer repräsentativen Studie für Deutschland zu dem Thema lag die Inanspruchnahme bei $52,9 \%$. Die am häufigsten benutzten komplementären Methoden durch CED-Patienten sind Homöopathie, Phythotherapie, Traditionelle Chinesische Medizin einschließlich Akupunktur, Diäten, Vitamine und Nahrungsergänzungsmittel. Prädiktoren für den Einsatz komplementärmedizinischer Verfahren sind ein höherer Bildungsstand, eine Vollwerternährung sowie eine kumulative Kortison-Tabletteneinnahme von mehr als $10 \mathrm{~g}$ Gesamtmenge. Ein höherer Body-Mass-Index (BMI) war negativ mit dem Einsatz von CAM verbunden.

Drei von vier Patienten gaben dabei Erfahrung mit mehr als einem Verfahren an. Nur 25\% der Patienten fühlten sich ausreichend über komplementäre Verfahren aufgeklärt. Bis zu 80\% gaben aber Interesse am Einsatz von komplementären Verfahren in der Zukunft an.

$30-70 \%$ der Patienten informiert ihre Ärzte/innen nicht über die Anwendung komplementärer Heilmethoden. Die Anwendung und das Verschweigen komplementärer Heilmethoden wird durch die konventionell behandelnden Ärzte unterschätzt. Als Gründe für die Anwendung komplementär-medizinischer Verfahren wurden in den Studien die Suche nach der optimalen Therapie, der Wunsch ohne Kortison auszukommen,
Nebenwirkungen der konventionellen Therapie, der Wunsch nach Stärkung der Eigenaktivität und der Eigenverantwortung, ein ganzheitlicher Therapieansatz sowie Unzufriedenheit mit der konventionellen Therapie und (relatives) Therapieversagen genannt. Bei Kindern korrelierte der Gebrauch von CAM mit der Zunahme von Fehlstunden in der Schule, Internetgebrauch und einem schlechterem Verlauf der CED.

\section{Empfehlung 10.15}

Curcumin kann in der remissionserhaltenden Behandlung komplementär zu 5-ASA eingesetzt werden.

[Evidenzgrad: B, Konsensusstärke: Konsens, Empfehlungsstärke: $\uparrow]$

\section{Erläuterung}

In einer prospektiv, randomisierten, doppelblinden, placebokontrollierten Multicenterstudie [705] wurde $2 \times 1 \mathrm{~g} / \mathrm{d}$ Curcumin komplementär zu Sulfasalazin oder Mesalazin in der remissionserhaltenden Therapie bei Colitis ulcerosa über einen Zeitraum von 6 Monaten untersucht (22 Patienten in der Verumgruppe). Es zeigte sich ein signifikanter Gruppenunterschied in Hinblick auf die Rezidivhäufigkeit, CAI und einem endoskopischen Index zugunsten der Verumgruppe zum Therapieende. Es zeigte sich kein Unterschied im Hinblick auf die Nebenwirkungen.

\section{Empfehlung 10.16}

Plantago ovata kann in der remissionserhaltenden Behandlung eingesetzt werden.

[Evidenzgrad: B, Konsensusstärke: Konsens, Empfehlungsstärke: $\uparrow]$

\section{Erläuterung}

In einer 3-armigen prospektiv, randomisierten, aktiv-kontrollierten, Open-label-Studie [706] wurden eine remissionserhaltende Therapie mit Plantago ovata $(2 \times 10 \mathrm{~g} / \mathrm{d})$, mit Mesalazin $(3 \times 500 \mathrm{mg} / \mathrm{d})$ und einer Kombinationstherapie aus beiden Medikamenten in einer Gruppe von 102 Patienten untersucht. Zum primären Endpunkt der Studie in Form der Remissionserhaltung nach 12 Monaten zeigte sich kein signifikanter Gruppenunterschied.

\section{Empfehlung 10.17}

Mind-Body-Therapie kann komplementär zur Verbesserung der Lebensqualität eingesetzt werden.

[Evidenzgrad: B, Konsensusstärke: Konsens, Empfehlungsstärke: $\uparrow]$

\section{Erläuterung}

In einer prospektiv, randomisiert, kontrollierten Studie im Wartegruppendesign $[682,683]$ konnten mit einem Lebensstilmodifikationsprogramm (Bewegung, Ernährung, Selbsthilfestrategien, Stressmanagement) über 10 Wochen bei Patienten in Remission oder mit geringer Krankheitsaktivität eine durchschnittliche Verbesserung des IBDQ zum Therapieende um 20 Punkte in der Interventionsgruppe erreicht werden (eine individuelle Verbesserung von 16 Punkten wird als klinisch relevant beurteilt). Der Psychische Summenscore im SF36 zeigte eine signifikante Verbesserung im Vergleich zur Wartekontroll- 
gruppe. In der Katamnese nach 12 Monaten ließ sich kein signifikanter Gruppenunterschied mehr nachweisen.

\section{Empfehlung 10.18}

Akupunktur (mit Moxibustion) kann im leicht bis moderaten Schub komplementär in der Therapie eingesetzt werden.

[Evidenzgrad: B, Konsensusstärke: Konsens, Empfehlungsstärke: $\uparrow$ ]

\section{Erläuterung}

In einer prospektiv, randomisierten und kontrollierten Studie [707] wurde die Akupunktur und Moxibustion (10 Sitzungen in 5 Wochen) bei leicht bis moderat aktiver Colitis ulcerosa im Vergleich zu oberflächlicher Nadelung an Nicht-Akupunkturpunkten untersucht.

Der CAI nahm nach der Akupunkturbehandlung gegenüber der Kontrollgruppe signifikant ab. Die sekundären Endpunkte: Verbesserung der Lebensqualität und Verbesserung des Allgemeinzustands zeigten keinen signifikanten Gruppenunterschied zum Ende der Therapie.

Eine Metaanalyse in chinesischer Sprache [708] zur Akupunktur liegt vor (Abstrakt auf Englisch) - mit positivem Outcome für Akupunktur bei insgesamt niedriger Bewertung der Qualität der eingeschlossenen Studien.

\section{Empfehlung 10.19}

Trichuris suis ovata (TSO) kann im leicht bis moderaten Schub die Krankheitsaktivität senken. Das Präparat ist in Deutschland nicht zugelassen. Eine generelle Empfehlung kann derzeit nicht gegeben werden.

[Evidenzgrad: B, Konsensusstärke: starker Konsens, Empfehlungsstärke: $\leftarrow \rightarrow$ ]

\section{Erläuterung}

In einer prospektiv, randomisierten, doppelblinden, placebokontrollierten monozentrischen Studie [709] wurden eine Therapie mit 2500 Trichuris-suis-Eiern alle 2 Wochen über 12 Wochen bei aktiver Colitis ulcerosa (CAI >4) untersucht. Zum Therapieende zeigte sich ein signifikanter Gruppenunterschied in Hinblick auf den CAI zugunsten der Verumgruppe. Es zeigten sich keine schwerwiegenden Nebeneffekte. Das Präparat ist in Deutschland als Medikament nicht zugelassen.

\section{Empfehlung 10.20}

Eine Empfehlung für die Anwendung weiterer unkonventioneller Verfahren kann nicht gegeben werden, da die Datenlage unzureichend ist.

[Evidenzgrad: D, Konsensusstärke: starker Konsens, Empfehlungsstärke: $\leftarrow \rightarrow$ ]

\section{Erläuterung}

In einer einzigen, lediglich in chinesisch publizierten randomisierten, doppelblind kontrollierten Studie [710] mit 153 Colitisulcerosa-Patienten wird eine Therapie mit traditionell chinesischer Medizin (Jian-Pi-Ling-Tabletten) signifikant wirksamer beschrieben als in den beiden Kontrollgruppen (behandelt mit
SASP in Kombination mit Dexamethason-Einläufen und Placebo). Für Tormentill (Blutwurz) liegt eine Open-label-Dosis-Eskalationsstudie [711] vor.

Eine offene, nicht randomisierte Studie [712, 713] zu Weihrauch mit 50 Patienten mit Colitis ulcerosa konnte keinen Unterschied zwischen Boswellia-serrata-Gummiharz und Sulfasalazin nachweisen.

In einer prospektiv, randomisierten, doppelblinden, placebokontrollierten Studie [714] wurde $100 \mathrm{ml}$ Aloe-vera-Gel über 4 Wochen (30 Pat. Verum : 14 Placebo) bei leicht bis mäßiggradiger Colitis ulcerosa untersucht. Aufgrund statistischer Mängel der Studie kann daraus eine Therapieempfehlung nicht abgeleitet werden.

\section{Empfehlung 10.21}

Aufgrund des hohen Anteils an Patienten, die komplementärmedizinische Therapien anwenden, sollten Ärzte sich über diese Verfahren informieren.

[Evidenzgrad: D, Konsensusstärke: mehrheitliche Zustimmung, Empfehlungsstärke: $\uparrow$ ]

\section{Erläuterung}

Ursache einer Anwendung von CAM durch Betroffene ist u.a. eine Unzufriedenheit mit der konventionellen Therapie (s.o.). Viele Betroffene sehen sich nicht durch die naturwissenschaftlich orientierte konventionelle Medizin in ihrer komplexen mehrdimensionalen Persönlichkeit erfasst. Die Auffassungen von Arzt und Patient zum Krankheitsverständnis und den Umgang mit der Erkrankung, wie auch zum Selbstbild und Weltbild, unterscheiden sich häufig. So kommt es dazu, dass außerhalb der ärztlichen Versorgung Beratung und Hilfe von CAMTherapeuten gesucht wird (z.B. bei Heilpraktikern). Nicht selten verfahren die Betroffenen dabei ,zweigleisig; Der Facharzt therapiert konventionell und der Patient sucht parallel nach weiteren Therapiemöglichkeiten, ohne dass der Arzt Kenntnis von den zusätzlich angewandten CAM-Verfahren erhält und der CAM-Therapeut entbehrt den speziellen medizinischen Sachverstand bei Colitis ulcerosa. 15-50\% der CED-Patienten berichten ihren behandelnden Ärzten nicht vom Gebrauch der komplementären Therapie aus Angst vor herabsetzender Bewertung durch den Arzt. Andererseits fragen weniger als 20\% der behandelnden spezialisierten CED-Ärzte den Patienten nach dem Gebrauch von CAM. Um hier Gefahren zu minimieren, sollte eine 'Zweigleisigkeit' der Therapie durch den Betroffenen vermieden werden und eine enge Abstimmung der Therapieverfahren erfolgen. Dazu sind ausreichende Kenntnisse der komplementären Therapieverfahren beim primär behandelnden Arzt erforderlich. Nur eine kompetente und sachliche Beratung auch auf dem Gebiet von CAM durch den primär behandelnden Arzt kann diese Zweigleisigkeit für den Patienten vermeiden. Idealerweise ist ein integratives Konzept anzustreben, bei dem konventionelle und komplementäre Therapieverfahren zu einer ,best practice' verschmelzen (WHO-Definition von integrativer Medizin). 


\section{Affiliations}

Medizinische Klinik I, Gastroent., Hepatologie, Onkologie und Infektiologie, Agaplesion Markus Krankenhaus, Frankfurt/Main

2 Medizinische Klinik I, Charité - Universitätsmedizin Berlin, Campus Benjamin Franklin

3 Universitätsklinikum Dresden, Institut für Pathologie

4 Klinikum Am Gesundbrunnen, Heilbronn, Institut für Pathologie

Zentrum für Kinder- und Jugendmedizin, Helios-Klinikum Krefeld

Gastroenterologische Gemeinschaftspraxis, Minden

Klinik und Poliklinik für Chirurgie, Universitätsklinikum Regensburg

8 Klinik für Innere Medizin II, Abt. Gastroenterologie, Hepatologie, Infektiologie, Universitätsklinikum Jena

9 Abteilung Innere Medizin I mit Schwerpunkt Gastroenterologie, RobertBosch-Krankenhaus, Stuttgart

10 Klinik für Psychosomatik und Psychotherapie, Universitätsklinikum Lübeck

11 Chirurgische Klinik, Krankenhaus Porz am Rhein

12 Abteilung Innere Medizin, Evang. Krankenhaus Kalk

13 Klinik für Allgemeine Innere Medizin und Gastroenterologie, Städtisches Klinikum Lüneburg

14 Innere V, Kliniken Essen-Mitte, Knappschafts-Krankenhaus

15 Medizinische Klinik I mit Schwerpunkt Gastroenterologie, Städtisches Klinikum Braunschweig

16 Klinik für Gastroenterologie und Hepatologie, Department für Innere Medizin, Universitätsspital Zürich

17 Medizinische Klinik I, St. Marien-Krankenhaus, Ludwigshafen

18 I. Medizinische Klinik, Klinik für Allgemeine Innere Medizin, Univ.-Klinikum Schleswig Holstein, Campus Kiel

\section{Literatur}

1 Timmer A, Goebell $H$. Incidence of ulcerative colitis, 1980-1995 - a prospective study in an urban population in Germany. Z Gastroenterol 1999; 37: 1079-1084

2 Ott C, Obermeier F, Thieler $S$ et al. The incidence of inflammatory bowel disease in a rural region of Southern Germany: a prospective population-based study. Eur J Gastroenterol Hepatol 2008; 20: 917923

3 Loftus EV. Clinical epidemiology of inflammatory bowel disease: incidence, prevalence, and environmental influences. Gastroenterology 2004; 126: 1504-1517

4 Lowe AM, Roy PO, B-Poulin M et al. Epidemiology of Crohn's disease in Quebec, Canada. Inflamm Bowel Dis 2009; 15: 429-435

5 Stark R, Konig HH, Leidl R. Costs of inflammatory bowel disease in Germany. Pharmacoeconomics 2006; 24: 797-814

6 Prenzler A, Bokemeyer B, Mittendorf T et al. Kosten der Colitis ulcerosa - eine Kalkulation aus der Perspektive der Gesetzlichen Krankenversicherung. Dtsch Med Wochenschr 2010; 135: 281-286

7 Hoffmann JC, Zeitz M, Bischoff SC et al. Diagnostik und Therapie der Colitis Ulcerosa: Ergebnisse einer evidenzbasierten Konsensuskonferenz der Deutschen Gesellschaft für Verdauungs- und Stoffwechselerkrankungen zusammen mit dem Kompetenznetz chronisch entzündliche Darmerkrankungen. Z Gastroenterol 2004; 42: 979-1042

8 Stange EF, Travis SP, Vermeire S et al. European evidence-based Consensus on the diagnosis and management of ulcerative colitis: Definitions and diagnosis. J Crohns Colitis 2008; 2: 1-23

9 Travis SP, Stange EF, Lemann $M$ et al. European evidence-based Consensus on the management of ulcerative colitis: Current management. J Crohns Colitis 2008; 2: 24-62

10 Biancone L, Michetti P, Travis S et al. European evidence-based Consensus on the management of ulcerative colitis: Special situations. J Crohns Colitis 2008; 2: 63-92

11 Arbeitsgemeinschaft der Wissenschaftlichen Medizinischen Fachgesellschaften ÄZQeditors. Deutsches Instrument zur methodischen Leitlinien-Bewertung (DELBI) - Fassung 2005/2006 + Domäne 8 (2008). Berlin 2008: Available from:www.delbi.de

12 Marteau P, Probert CS, Lindgren S et al. Combined oral and enema treatment with Pentasa (mesalazine) is superior to oral therapy alone in patients with extensive mild/moderate active ulcerative colitis: a randomised, double blind, placebo controlled study. Gut 2005; 54 : 960-965

13 Silverberg MS, Satsangi J, Ahmad T et al. Toward an integrated clinical, molecular and serological classification of inflammatory bowel disease: Report of a Working Party of the 2005 Montreal World Congress of Gastroenterology. Can J Gastroenterol 2005; 19 (Suppl A): 5-36

14 Ekbom A, Helmick C, Zack M et al. Ulcerative colitis and colorectal cancer. A population-based study. N Engl J Med 1990; 323: 1228-1233
15 Soetikno RM, Lin OS, Heidenreich PA et al. Increased risk of colorectal neoplasia in patients with primary sclerosing cholangitis and ulcerative colitis: a meta-analysis. Gastrointest Endosc 2002; 56: 48-54

16 Forrest $K$, Symmons D, Foster P. Systematic review: is ingestion of paracetamol or non-steroidal anti-inflammatory drugs associated with exacerbations of inflammatory bowel disease? Aliment Pharmacol Ther 2004; 20: 1035-1043

17 Takeuchi K, Smale S, Premchand P et al. Prevalence and mechanism of nonsteroidal anti-inflammatory drug-induced clinical relapse in patients with inflammatory bowel disease. Clin Gastroenterol Hepatol 2006; 4: 196-202

18 Sandborn WJ, Stenson WF, Brynskov J et al. Safety of celecoxib in patients with ulcerative colitis in remission: a randomized, placebocontrolled, pilot study. Clin Gastroenterol Hepatol 2006; 4: 203-211

19 Korzenik JR, Podolsky DK. Selective use of selective nonsteroidal antiinflammatory drugs in inflammatory bowel disease. Clin Gastroenterol Hepatol 2006; 4: 157-159

20 Ho GT, Chiam P, Drummond H et al. The efficacy of corticosteroid therapy in inflammatory bowel disease: analysis of a 5-year UK inception cohort. Aliment Pharmacol Ther 2006; 24: 319-330

21 FaubionJr WA, LoftusJr EV, Harmsen WS et al. The natural history of corticosteroid therapy for inflammatory bowel disease: a population-based study. Gastroenterology 2001; 121: 255-260

22 Toruner M, LoftusJr EV, Harmsen WS et al. Risk factors for opportunistic infections in patients with inflammatory bowel disease. Gastroenterology 2008; 134: 929-936

23 Mahid SS, Minor KS, Soto RE et al. Smoking and inflammatory bowel disease: a meta-analysis. Mayo Clin Proc 2006; 81: 1462-1471

24 Hoie 0 , Wolters $F$, Riis L et al. Ulcerative colitis: patient characteristics may predict 10-yr disease recurrence in a European-wide population-based cohort. Am J Gastroenterol 2007; 102: 1692-1701

25 Beaugerie L, Massot N, Carbonnel F et al. Impact of cessation of smoking on the course of ulcerative colitis. Am J Gastroenterol 2001; 96: 2113-2116

26 Birrenbach T, Bocker U. Inflammatory bowel disease and smoking: a review of epidemiology, pathophysiology, and therapeutic implications. Inflamm Bowel Dis 2004; 10: 848-859

27 Rudra T, Motley R, Rhodes J. Does smoking improve colitis? Scand J Gastroenterol Suppl 1989; 170: 61-63; discussion 66-68

28 LoftusJr EV, Sandborn WJ, Tremaine WJ et al. Primary sclerosing cholangitis is associated with nonsmoking: a case-control study. Gastroenterology 1996; 110: 1496-1502

29 Merrett MN, Mortensen $N$, Kettlewell $M$ et al. Smoking may prevent pouchitis in patients with restorative proctocolectomy for ulcerative colitis. Gut 1996; 38: 362-364

30 Joelsson $M$, Benoni $C$, Oresland $T$. Does smoking influence the risk of pouchitis following ileal pouch anal anastomosis for ulcerative colitis? Scand J Gastroenterol 2006; 41: 929-933

31 LoftusJr EV. Clinical epidemiology of inflammatory bowel disease: Incidence, prevalence, and environmental influences. Gastroenterology 2004; 126: 1504-1517

32 Rutgeerts $P$, D'Haens $G$, Hiele $M$ et al. Appendectomy protects against ulcerative colitis. Gastroenterology 1994; 106: 1251-1253

33 Frisch $M$, Johansen $C$, Mellemkjaer L et al. Appendectomy and subsequent risk of inflammatory bowel diseases. Surgery 2001; 130: 36-43

34 Koutroubakis IE, Vlachonikolis IG, Kouroumalis EA. Role of appendicitis and appendectomy in the pathogenesis of ulcerative colitis: a critical review. Inflamm Bowel Dis 2002; 8: 277-286

35 Cosnes J, Carbonnel F, Beaugerie L et al. Effects of appendicectomy on the course of ulcerative colitis. Gut 2002; 51: 803-807

36 Radford-Smith GL, Edwards JE, Purdie DM et al. Protective role of appendicectomy on onset and severity of ulcerative colitis and Crohn's disease. Gut 2002; 51: 808-813

37 Florin TH, Pandeya N, Radford-Smith GL. Epidemiology of appendicectomy in primary sclerosing cholangitis and ulcerative colitis: its influence on the clinical behaviour of these diseases. Gut 2004; 53: 973-979

38 Vermeire $S$. Review article: genetic susceptibility and application of genetic testing in clinical management of inflammatory bowel disease. Aliment Pharmacol Ther 2006; 24 (Suppl 3): 2-10

39 Van Kruiningen HJ, Joossens M, Vermeire S et al. Familial Crohn's disease in Belgium: pedigrees, temporal relationships among cases, and family histories. J Clin Gastroenterol 2007; 41: 583-590

40 Hanauer SB. Update on the etiology, pathogenesis and diagnosis of ulcerative colitis. Nat Clin Pract Gastroenterol Hepatol 2004; 1: 26-31 
41 Xavier RJ, Podolsky DK. Unravelling the pathogenesis of inflammatory bowel disease. Nature 2007; 448: 427-434

42 Bernstein $C N$, Rawsthorne $P$, Cheang $M$ et al. A population-based case control study of potential risk factors for IBD. Am J Gastroenterol 2006; 101: 993-1002

43 Garcia Rodriguez LA, Gonzalez-Perez A, Johansson S et al. Risk factors for inflammatory bowel disease in the general population. Aliment Pharmacol Ther 2005; 22: 309-315

44 Henriksen M, Jahnsen J, Lygren I et al. Are there any differences in phenotype or disease course between familial and sporadic cases of inflammatory bowel disease? Results of a population-based follow-up study. Am J Gastroenterol 2007; 102: 1955-1963

45 IBD Working Group of the ESPGHN. Inflammatory bowel disease in children and adolescents: recommendations for diagnosis - the Porto criteria. J Pediatr Gastroenterol Nutr 2005; 41: 1-7

46 Langholz E, Munkholm P, Davidsen M et al. Course of ulcerative colitis: analysis of changes in disease activity over years. Gastroenterology 1994; 107: 3-11

47 Henriksen M, Jahnsen J, Lygren I et al. Change of diagnosis during the first five years after onset of inflammatory bowel disease: results of a prospective follow-up study (the IBSEN Study). Scand J Gastroenterol 2006; 41: 1037-1043

48 Price $A B$. Overlap in the spectrum of non-specific inflammatory bowel disease - 'colitis indeterminate'. J Clin Pathol 1978; 31: 567-577

49 Rodgers AD, Cummins AG. CRP correlates with clinical score in ulcerative colitis but not in Crohn's disease. Dig Dis Sci 2007; 52: 20632068

50 Vermeire S, Van Assche G, Rutgeerts P. C-reactive protein as a marker for inflammatory bowel disease. Inflamm Bowel Dis 2004; 10: 661665

51 Prantera C, Davoli M, Lorenzetti R et al. Clinical and laboratory indicators of extent of ulcerative colitis. Serum C-reactive protein helps the most. J Clin Gastroenterol 1988; 10: 41-45

52 Korczowski B, Szybist W. Serum procalcitonin and C-reactive protein in children with diarrhoea of various aetiologies. Acta Paediatr 2004; 93: $169-173$

53 Herrlinger KR, Dittmann $R$, Weitz G et al. Serum procalcitonin differentiates inflammatory bowel disease and self-limited colitis. Inflamm Bowel Dis 2004; 10: 229-233

54 Mylonaki M, Langmead L, Pantes A et al. Enteric infection in relapse of inflammatory bowel disease: importance of microbiological examination of stool. Eur J Gastroenterol Hepatol 2004; 16: 775-778

55 Ajaj WM, Lauenstein TC, Pelster G et al. Magnetic resonance colonography for the detection of inflammatory diseases of the large bowel: quantifying the inflammatory activity. Gut 2005; 54: 257-263

56 Roseth AG, Aadland E, Grzyb K. Normalization of faecal calprotectin: a predictor of mucosal healing in patients with inflammatory bowel disease. Scand J Gastroenterol 2004; 39: 1017-1020

57 Schoepfer AM, Beglinger C, Straumann A et al. Ulcerative colitis: Correlation of the Rachmilewitz endoscopic activity index with fecal calprotectin, clinical activity, C-reactive protein, and blood leukocytes. Inflamm Bowel Dis 2009; 15: 1851-1858

58 Plevy S. Do serological markers and cytokines determine the indeterminate? J Clin Gastroenterol 2004; 38: S51-56

59 Riis L, Vind I, Vermeire S et al. The prevalence of genetic and serologic markers in an unselected European population-based cohort of IBD patients. Inflamm Bowel Dis 2007; 13: 24-32

60 Joossens S, Daperno M, Shums Z et al. Interassay and interobserver variability in the detection of anti-neutrophil cytoplasmic antibodies in patients with ulcerative colitis. Clin Chem 2004; 50: 1422-1425

61 Reese GE, Constantinides VA, Simillis C et al. Diagnostic precision of anti-Saccharomyces cerevisiae antibodies and perinuclear antineutrophil cytoplasmic antibodies in inflammatory bowel disease. Am J Gastroenterol 2006; 101: 2410-2422

62 Brown WJ, Hudson MJ, Patrick S et al. Search for enteric microbial pathogens in patients with ulcerative colitis. Digestion 1992; 53: 121128

63 Weber P, Koch M, Heizmann WR et al. Microbic superinfection in relapse of inflammatory bowel disease. J Clin Gastroenterol 1992; 14: 302-308

64 Rolny P, Jarnerot G, Mollby R. Occurrence of Clostridium difficile toxin in inflammatory bowel disease. Scand J Gastroenterol 1983; 18: 61 64
65 Issa M, Vijayapal A, Graham MB et al. Impact of Clostridium difficile on inflammatory bowel disease. Clin Gastroenterol Hepatol 2007; 5: 345-351

66 Rodemann JF, Dubberke ER, Reske KA et al. Incidence of Clostridium difficile infection in inflammatory bowel disease. Clin Gastroenterol Hepatol 2007; 5: 339-344

67 Johal SS, Hammond J, Solomon K et al. Clostridium difficile associated diarrhoea in hospitalised patients: onset in the community and hospital and role of flexible sigmoidoscopy. Gut 2004; 53: 673-677

68 Minami M, Ohta M, Ohkura T et al. Cytomegalovirus infection in severe ulcerative colitis patients undergoing continuous intravenous cyclosporine treatment in Japan. World J Gastroenterol 2007; 13: 754760

69 Matsuoka K, Iwao Y, Mori T et al. Cytomegalovirus is frequently reactivated and disappears without antiviral agents in ulcerative colitis patients. Am J Gastroenterol 2007; 102: 331-337

70 Dimitroulia E, Spanakis N, Konstantinidou AE et al. Frequent detection of cytomegalovirus in the intestine of patients with inflammatory bowel disease. Inflamm Bowel Dis 2006; 12: 879-884

71 Hommes DW, Sterringa G, van Deventer SJ et al. The pathogenicity of cytomegalovirus in inflammatory bowel disease: a systematic review and evidence-based recommendations for future research. Inflamm Bowel Dis 2004; 10: 245-250

72 Kojima T, Watanabe T, Hata K et al. Cytomegalovirus infection in ulcerative colitis. Scand J Gastroenterol 2006; 41: 706-711

73 Vermeire S, Van Assche G, Rutgeerts P. Laboratory markers in IBD: useful, magic, or unnecessary toys? Gut 2006; 55: 426-431

74 Poullis A, Foster R, Northfield TC et al. Review article: faecal markers in the assessment of activity in inflammatory bowel disease. Aliment Pharmacol Ther 2002; 16: 675-681

75 Langhorst J, Elsenbruch S, Mueller T et al. Comparison of 4 neutrophilderived proteins in feces as indicators of disease activity in ulcerative colitis. Inflamm Bowel Dis 2005; 11: 1085-1091

76 Konikoff MR, Denson LA. Role of fecal calprotectin as a biomarker of intestinal inflammation in inflammatory bowel disease. Inflamm Bowel Dis 2006; 12: 524-534

77 Fine KD, Ogunji F, George J et al. Utility of a rapid fecal latex agglutination test detecting the neutrophil protein, lactoferrin, for diagnosing inflammatory causes of chronic diarrhea. Am J Gastroenterol 1998; 93: $1300-1305$

78 D'Inca R, Dal Pont E, Di Leo V et al. Can calprotectin predict relapse risk in inflammatory bowel disease? Am J Gastroenterol 2008; 103 2007-2014

79 Costa F, Mumolo MG, Bellini M et al. Role of faecal calprotectin as noninvasive marker of intestinal inflammation. Dig Liver Dis 2003; 35: 642-647

80 Roseth AG, Aadland E, Jahnsen J et al. Assessment of disease activity in ulcerative colitis by faecal calprotectin, a novel granulocyte marker protein. Digestion 1997; 58: 176-180

81 de Jong NS, Leach ST, Day AS. Fecal S100A12: a novel noninvasive marker in children with Crohn's disease. Inflamm Bowel Dis 2006; 12 566-572

82 von Roon AC, Karamountzos L, Purkayastha S et al. Diagnostic precision of fecal calprotectin for inflammatory bowel disease and colorectal malignancy. Am J Gastroenterol 2007; 102: 803-813

83 Kaiser T, Langhorst J, Wittkowski H et al. Faecal S100A12 as a non-invasive marker distinguishing inflammatory bowel disease from irritable bowel syndrome. Gut 2007; 56: 1706-1713

84 Foell D, Wittkowski H, Roth J. Monitoring disease activity by stool analyses: from occult blood to molecular markers of intestinal inflammation and damage. Gut 2009; 58: 859-868

85 Walker TR, Land ML, Kartashov A et al. Fecal lactoferrin is a sensitive and specific marker of disease activity in children and young adults with inflammatory bowel disease. J Pediatr Gastroenterol Nutr 2007; 44: 414-422

86 Joishy $M$, Davies I, Ahmed $M$ et al. Fecal calprotectin and lactoferrin as noninvasive markers of pediatric inflammatory bowel disease. J Pediatr Gastroenterol Nutr 2009; 48: 48-54

87 Quail MA, Russell RK, Van Limbergen JE et al. Fecal calprotectin complements routine laboratory investigations in diagnosing childhood inflammatory bowel disease. Inflamm Bowel Dis 2009; 15: 756-759

88 Tibble JA, Sigthorsson G, Bridger S et al. Surrogate markers of intestinal inflammation are predictive of relapse in patients with inflammatory bowel disease. Gastroenterology 2000; 119: 15-22 
89 Costa F, Mumolo MG, Ceccarelli L et al. Calprotectin is a stronger predictive marker of relapse in ulcerative colitis than in Crohn's disease. Gut 2005; 54: 364-368

90 Carbonnel F, Lavergne A, Lemann $M$ et al. Colonoscopy of acute colitis. A safe and reliable tool for assessment of severity. Dig Dis Sci 1994; 39: 1550-1557

91 Alemayehu G, Jarnerot G. Colonoscopy during an attack of severe ulcerative colitis is a safe procedure and of great value in clinical decision making. Am J Gastroenterol 1991; 86: 187-190

92 Fefferman DS, Farrell RJ. Endoscopy in inflammatory bowel disease: indications, surveillance, and use in clinical practice. Clin Gastroenterol Hepatol 2005; 3: 11-24

93 Deutsch $D E$, Olson $A D$. Colonoscopy or sigmoidoscopy as the initial evaluation of pediatric patients with colitis: a survey of physician behavior and a cost analysis. J Pediatr Gastroenterol Nutr 1997; 25: 2631

94 Langhorst J, Kuhle CA, Ajaj W et al. MR colonography without bowel purgation for the assessment of inflammatory bowel diseases: diagnostic accuracy and patient acceptance. Inflamm Bowel Dis 2007; 13: $1001-1008$

95 Schreyer AG, Scheibl K, Heiss P et al. MR colonography in inflammatory bowel disease. Abdom Imaging 2006; 31: 302-307

96 Triester SL, Leighton JA, Leontiadis GI et al. A meta-analysis of the yield of capsule endoscopy compared to other diagnostic modalities in patients with non-stricturing small bowel Crohn's disease. Am J Gastroenterol 2006; 101: 954-964

97 Waye JD. The role of colonoscopy in the differential diagnosis of inflammatory bowel disease. Gastrointest Endosc 1977; 23: 150-154

98 Floren $\mathrm{CH}$, Benoni $\mathrm{C}$, Willen $\mathrm{R}$. Histologic and colonoscopic assessment of disease extension in ulcerative colitis. Scand J Gastroenterol 1987; 22: 459-462

99 Pera A, Bellando P, Caldera D et al. Colonoscopy in inflammatory bowel disease. Diagnostic accuracy and proposal of an endoscopic score. Gastroenterology 1987; 92: 181-185

100 Kiesslich R, Fritsch J, Holtmann M et al. Methylene blue-aided chromoendoscopy for the detection of intraepithelial neoplasia and colon cancer in ulcerative colitis. Gastroenterology 2003; 124: 880-888

101 Pineton de Chambrun G, Peyrin-Biroulet L, Lemann M et al. Clinical implications of mucosal healing for the management of IBD. Nat Rev Gastroenterol Hepatol 2010; 7: 15-29

102 Parente F, Molteni M, Marino B et al. Bowel ultrasound and mucosal healing in ulcerative colitis. Dig Dis 2009; 27: 285-290

103 Froslie KF, Jahnsen J, Moum BA et al. Mucosal healing in inflammatory bowel disease: results from a Norwegian population-based cohort. Gastroenterology 2007; 133: 412-422

104 Hoffmann JC, Preiss JC, Autschbach F et al. Clinical practice guideline on diagnosis and treatment of Crohn's disease. Z Gastroenterol 2008; 46: 1094-1146

105 D'Haens G, Sandborn WJ, Feagan BG et al. A review of activity indices and efficacy end points for clinical trials of medical therapy in adults with ulcerative colitis. Gastroenterology 2007; 132: 763-786

106 Truelove SC, Witts LJ. Cortisone in ulcerative colitis; final report on a therapeutic trial. Br Med J 1955; 2: 1041-1048

107 Jakobovits SL, Travis SP. Management of acute severe colitis. Br Med Bull 2005; 75 - 76: 131-144

108 Turner D, Otley AR, Mack D et al. Development, validation, and evaluation of a pediatric ulcerative colitis activity index: a prospective multicenter study. Gastroenterology 2007; 133: 423-432

109 Parente F, Greco S, Molteni M et al. Role of early ultrasound in detecting inflammatory intestinal disorders and identifying their anatomical location within the bowel. Aliment Pharmacol Ther 2003; 18: 1009-1016

110 Hollerbach S, Geissler A, Schiegl H et al. The accuracy of abdominal ultrasound in the assessment of bowel disorders. Scand J Gastroenterol 1998; 33: 1201-1208

111 Maconi G, Ardizzone S, Parente F et al. Ultrasonography in the evaluation of extension, activity, and follow-up of ulcerative colitis. Scand J Gastroenterol 1999; 34: 1103-1107

112 Worlicek H, Lutz H, Heyder $N$ et al. Ultrasound findings in Crohn's disease and ulcerative colitis: a prospective study. J Clin Ultrasound 1987; 15: 153-163

113 Bozkurt T, Richter F, Lux G. Ultrasonography as a primary diagnostic tool in patients with inflammatory disease and tumors of the small intestine and large bowel. J Clin Ultrasound 1994; 22: 85-91
114 Arienti V, Campieri M, Boriani L et al. Management of severe ulcerative colitis with the help of high resolution ultrasonography. Am J Gastroenterol 1996; 91: 2163-2169

115 Faure C, Belarbi N, Mougenot JF et al. Ultrasonographic assessment of inflammatory bowel disease in children: comparison with ileocolonoscopy. J Pediatr 1997; 130: 147-151

116 Pradel JA, David XR, Taourel $P$ et al. Sonographic assessment of the normal and abnormal bowel wall in nondiverticular ileitis and colitis. Abdom Imaging 1997; 22: 167-172

117 Haber HP, Busch A, Ziebach R et al. Ultrasonographic findings correspond to clinical, endoscopic, and histologic findings in inflammatory bowel disease and other enterocolitides. J Ultrasound Med 2002; 21: 375-382

118 Pascu M, Roznowski AB, Muller HP et al. Clinical relevance of transabdominal ultrasonography and magnetic resonance imaging in patients with inflammatory bowel disease of the terminal ileum and large bowel. Inflamm Bowel Dis 2004; 10: 373-382

119 Parente F, Molteni M, Marino B et al. Are Colonoscopy and Bowel Ultrasound Useful for Assessing Response to Short-Term Therapy and Predicting Disease Outcome of Moderate-to-Severe Forms of Ulcerative Colitis? A Prospective Study. Am J Gastroenterol 2009; 105: 1150-1157

120 Dixit R, Chowdhury V, Kumar N. Hydrocolonic sonography in the evaluation of colonic lesions. Abdom Imaging 1999; 24: 497-505

121 Ludwig D, Wiener S, Bruning A et al. Mesenteric blood flow is related to disease activity and risk of relapse in ulcerative colitis: a prospective follow up study. Gut 1999; 45: 546-552

122 Homann N, Klarmann U, Fellermann K et al. Mesenteric pulsatility index analysis predicts response to azathioprine in patients with Crohn's disease. Inflamm Bowel Dis 2005; 11: 126-132

123 Rutter MD, Saunders BP, Wilkinson KH et al. Cancer surveillance in longstanding ulcerative colitis: endoscopic appearances help predict cancer risk. Gut 2004; 53: 1813-1816

124 Andersen K, Vogt C, Blondin D et al. Multi-detector CT-colonography in inflammatory bowel disease: prospective analysis of CT-findings to high-resolution video colonoscopy. Eur J Radiol 2006; 58: 140-146

125 Horsthuis $K$, Bipat S, Bennink RJ et al. Inflammatory bowel disease diagnosed with US, MR, scintigraphy, and CT: meta-analysis of prospective studies. Radiology 2008; 247: 64-79

126 Sawczenko A, Sandhu BK. Presenting features of inflammatory bowe disease in Great Britain and Ireland. Arch Dis Child 2003; 88: 995 1000

127 Allison MC, Hamilton-Dutoit SJ, Dhillon AP et al. The value of recta biopsy in distinguishing self-limited colitis from early inflammatory bowel disease. Q J Med 1987; 65: 985-995

128 Bentley E, Jenkins D, Campbell F et al. How could pathologists improve the initial diagnosis of colitis? Evidence from an international workshop. J Clin Pathol 2002; 55: 955-960

129 Dejaco C, Oesterreicher C, Angelberger S et al. Diagnosing colitis: a prospective study on essential parameters for reaching a diagnosis. Endoscopy 2003; 35: 1004-1008

130 Dube AK, Cross SS, Lobo AJ. Audit of the histopathological diagnosis of non-neoplastic colorectal biopsies: achievable standards for the diagnosis of inflammatory bowel disease. J Clin Pathol 1998; 51: 378-381

131 Dundas SA, Dutton J, Skipworth P. Reliability of rectal biopsy in distinguishing between chronic inflammatory bowel disease and acute self-limiting colitis. Histopathology 1997; 31: 60-66

132 Jenkins D, Balsitis M, Gallivan S et al. Guidelines for the initial biopsy diagnosis of suspected chronic idiopathic inflammatory bowel disease. The British Society of Gastroenterology Initiative. J Clin Pathol 1997; 50: 93-105

133 Nostrant TT, Kumar NB, Appelman HD. Histopathology differentiates acute self-limited colitis from ulcerative colitis. Gastroenterology 1987; 92: 318-328

134 Schumacher G, Kollberg B, Sandstedt B. A prospective study of first attacks of inflammatory bowel disease and infectious colitis. Histologic course during the 1st year after presentation. Scand J Gastroenterol 1994: 29: 318-332

135 Seldenrijk CA, Morson BC, Meuwissen SG et al. Histopathological evaluation of colonic mucosal biopsy specimens in chronic inflammatory bowel disease: diagnostic implications. Gut 1991; 32: 1514-1520

136 Surawicz CM, Belic L. Rectal biopsy helps to distinguish acute selflimited colitis from idiopathic inflammatory bowel disease. Gastroenterology 1984; 86: 104-113 
137 Tanaka M, Masuda T, Yao T et al. Observer variation of diagnoses based on simple biopsy criteria differentiating among Crohn's disease, ulcerative colitis, and other forms of colitis. J Gastroenterol Hepatol 2001; 16: 1368-1372

138 Tanaka M, Riddell $\mathrm{RH}$, Saito $\mathrm{H}$ et al. Morphologic criteria applicable to biopsy specimens for effective distinction of inflammatory bowel disease from other forms of colitis and of Crohn's disease from ulcerative colitis. Scand J Gastroenterol 1999; 34: 55-67

139 Tanaka M, Saito H, Fukuda S et al. Simple mucosal biopsy criteria differentiating among Crohn disease, ulcerative colitis, and other forms of colitis: measurement of validity. Scand J Gastroenterol 2000; 35: 281-286

140 Theodossi A, Spiegelhalter DJ, Jass J et al. Observer variation and discriminatory value of biopsy features in inflammatory bowel disease. Gut 1994; 35: 961-968

141 McHugh JB, Appelman HD, McKenna BJ. The diagnostic value of endoscopic terminal ileum biopsies. Am J Gastroenterol 2007; 102: 10841089

142 Cherian S, Singh P. Is routine ileoscopy useful? An observational study of procedure times, diagnostic yield, and learning curve. Am J Gastroenterol 2004; 99: 2324-2329

143 Geboes K, Ectors N, D'Haens G et al. Is ileoscopy with biopsy worthwhile in patients presenting with symptoms of inflammatory bowel disease? Am J Gastroenterol 1998; 93: 201-206

144 Lemberg DA, Clarkson CM, Bohane TD et al. Role of esophagogastroduodenoscopy in the initial assessment of children with inflammatory bowel disease. J Gastroenterol Hepatol 2005; 20: 1696-1700

145 Surawicz CM. Serial sectioning of a portion of a rectal biopsy detects more focal abnormalities: a prospective study of patients with inflammatory bowel disease. Dig Dis Sci 1982; 27: 434-436

146 Surawicz CM, Meisel JL, Ylvisaker T et al. Rectal biopsy in the diagnosis of Crohn's disease: value of multiple biopsies and serial sectioning. Gastroenterology 1981; 80: 66-71

147 Therkildsen MH, Jensen BN, Teglbjaerg PS et al. The final outcome of patients presenting with their first episode of acute diarrhoea and an inflamed rectal mucosa with preserved crypt architecture. A clinicopathologic study. Scand J Gastroenterol 1989; 24: 158-164

148 Washington K, Greenson JK, Montgomery E et al. Histopathology of ulcerative colitis in initial rectal biopsy in children. Am J Surg Pathol 2002; 26: 1441-1449

149 Geboes K, Dalle I. Influence of treatment on morphological features of mucosal inflammation. Gut 2002; 50 (Suppl 3): III37-III42

150 Odze R, Antonioli D, Peppercorn $M$ et al. Effect of topical 5-aminosalicylic acid (5-ASA) therapy on rectal mucosal biopsy morphology in chronic ulcerative colitis. Am J Surg Pathol 1993; 17: 869-875

151 Kleer CG, Appelman HD. Ulcerative colitis: patterns of involvement in colorectal biopsies and changes with time. Am J Surg Pathol 1998; 22: 983-989

152 Bousvaros A, Antonioli DA, Colletti RB et al. Differentiating ulcerative colitis from Crohn disease in children and young adults: report of a working group of the North American Society for Pediatric Gastroenterology, Hepatology, and Nutrition and the Crohn's and Colitis Foundation of America. J Pediatr Gastroenterol Nutr 2007; 44: 653-674

153 Glickman JN, Bousvaros A, Farraye FA et al. Pediatric patients with untreated ulcerative colitis may present initially with unusual morphologic findings. Am J Surg Pathol 2004; 28: 190-197

154 Robert ME, Tang L, Hao LM et al. Patterns of inflammation in mucosal biopsies of ulcerative colitis: perceived differences in pediatric populations are limited to children younger than 10 years. Am J Surg Pathol 2004; 28: 183-189

155 Markowitz J, Kahn E, Grancher K et al. Atypical rectosigmoid histology in children with newly diagnosed ulcerative colitis. Am J Gastroenterol 1993; 88: 2034-2037

156 Autschbach F. Pathologie der CED. In: Hoffmann JC, Krösen AJ, Klump $B$, eds. Chronisch entzündliche Darmerkrankungen Das CED-Handbuch für Klinik und Praxis. Stuttgart: Thieme, 2004: 110-122

157 Borchard F. Differential diagnosis of colitis. Verh Dtsch Ges Pathol 1999; 83: 110-121

158 von Herbay A. Histopathologische Diagnostik chronischentzündlicher Darmerkrankungen. Historischer Rückblick und aktuelle Übersicht. Pathologe 1999; 20: 276-287

159 Gomes P, du Boulay C, Smith CL et al. Relationship between disease activity indices and colonoscopic findings in patients with colonic inflammatory bowel disease. Gut 1986; 27: 92-95
160 D'Haens G, Van Deventer S, Van Hogezand R et al. Endoscopic and histological healing with infliximab anti-tumor necrosis factor antibodies in Crohn's disease: A European multicenter trial. Gastroenterology 1999; 116: 1029-1034

161 Riley SA, Mani V, Goodman MJ et al. Microscopic activity in ulcerative colitis: what does it mean? Gut 1991; 32: 174-178

162 Bitton A, Peppercorn MA, Antonioli DA et al. Clinical, biological, and histologic parameters as predictors of relapse in ulcerative colitis. Gastroenterology 2001; 120: 13-20

163 Nishio Y, Ando T, Maeda 0 et al. Pit patterns in rectal mucosa assessed by magnifying colonoscope are predictive of relapse in patients with quiescent ulcerative colitis. Gut 2006; 55: 1768-1773

164 Taylor BA, Pemberton JH, Carpenter HA et al. Dysplasia in chronic ulcerative colitis: implications for colonoscopic surveillance. Dis Colon Rectum 1992; 35: 950-956

165 Thomas T, Abrams KA, Robinson RJ et al. Meta-analysis: cancer risk of low-grade dysplasia in chronic ulcerative colitis. Alimentary Pharmacology \& Therapeutics 2007; 25: 657-668

166 Ullman T, Croog V, Harpaz $N$ et al. Progression of flat low-grade dysplasia to advanced neoplasia in patients with ulcerative colitis. Gastroenterology 2003; 125: 1311-1319

167 Befrits R, Ljung T, Jaramillo E et al. Low-grade dysplasia in extensive, long-standing inflammatory bowel disease: a follow-up study. Dis Colon Rectum 2002; 45: 615-620

168 Lim CH, Dixon MF, Vail A et al. Ten year follow up of ulcerative colitis patients with and without low grade dysplasia. Gut 2003; 52: 1127 1132

169 Pohl C, Hombach A, Kruis W. Chronic inflammatory bowel disease and cancer. Hepatogastroenterology 2000; 47: 57-70

170 Melville DM, Jass JR, Morson BC et al. Observer study of the grading of dysplasia in ulcerative colitis: comparison with clinical outcome. Hum Pathol 1989; 20: 1008-1014

171 Jess T, LoftusJr EV, Velayos FS et al. Incidence and prognosis of colorectal dysplasia in inflammatory bowel disease: a population-based study from Olmsted County, Minnesota. Inflamm Bowel Dis 2006; 12: 669-676

172 Bernstein CN, Shanahan F, Weinstein WM. Are we telling patients the truth about surveillance colonoscopy in ulcerative colitis? Lancet 1994; 343: 71-74

173 Blackstone MO, Riddell RH, Rogers BH et al. Dysplasia-associated lesion or mass (DALM) detected by colonoscopy in long-standing ulcerative colitis: an indication for colectomy. Gastroenterology 1981; 80: 366374

174 Engelsgjerd $M$, Farraye FA, Odze RD. Polypectomy may be adequate treatment for adenoma-like dysplastic lesions in chronic ulcerative colitis. Gastroenterology 1999; 117: 1288-1294; discussion 1488 1291

175 Rubin PH, Friedman S, Harpaz N et al. Colonoscopic polypectomy in chronic colitis: conservative management after endoscopic resection of dysplastic polyps. Gastroenterology 1999; 117: 1295-1300

176 Torres C, Antonioli D, Odze RD. Polypoid dysplasia and adenomas in inflammatory bowel disease: a clinical, pathologic, and follow-up study of 89 polyps from 59 patients. Am J Surg Pathol 1998; 22: 275-284

177 Vieth $M$, Behrens $H$, Stolte $M$. Sporadic adenoma in ulcerative colitis: endoscopic resection is an adequate treatment. Gut 2006; 55: 1151 1155

178 Schneider A, Stolte M. Differential diagnosis of adenomas and dysplastic lesions in patients with ulcerative colitis. Z Gastroenterol 1993; 31: 653-656

179 Campieri M, Gionchetti P, Belluzzi A et al. 5-Aminosalicylic acid as enemas or suppositories in distal ulcerative colitis? Journal of Clinical Gastroenterology 1988; 10: 406-409

180 Brown J, Haines S, Wilding IR. Colonic spread of three rectally administered mesalazine (Pentasa) dosage forms in healthy volunteers as assessed by gamma scintigraphy. Alimentary Pharmacology \& Therapeutics 1997; 11: 685-691

181 Campieri M, De Franchis R, Bianchi Porro G et al. Mesalazine (5-aminosalicylic acid) suppositories in the treatment of ulcerative proctitis or distal proctosigmoiditis. A randomized controlled trial. Scandinavian Journal of Gastroenterology 1990; 25: 663-668

182 Jacobsen BA, Abildgaard K, Rasmussen HH et al. Availability of mesalazine (5-aminosalicylic acid) from enemas and suppositories during steady-state conditions. Scandinavian Journal of Gastroenterology 1991; 26 : 374-378 
183 Marshall JK, Irvine EJ. Rectal aminosalicylate therapy for distal ulcerative colitis: a meta-analysis. Aliment Pharmacol Ther 1995; 9: 293300

184 Regueiro M, Loftus EV, Steinhart AH et al. Medical management of leftsided ulcerative colitis and ulcerative proctitis: critical evaluation of therapeutic trials. Inflammatory Bowel Diseases 2006; 12: 979-994

185 Lawrance IC, Copeland TS. Rectal tacrolimus in the treatment of resistant ulcerative proctitis. Alimentary Pharmacology \& Therapeutics 2008; 28 : $1214-1220$

186 van Dieren JM, van Bodegraven AA, Kuipers EJ et al. Local application of tacrolimus in distal colitis: feasible and safe. Inflammatory Bowel Diseases 2009; 15: 193-198

187 Safdi M, DeMicco M, Sninsky C et al. A double-blind comparison of oral versus rectal mesalamine versus combination therapy in the treatment of distal ulcerative colitis. The American Journal of Gastroenterology 1997; 92: 1867-1871

188 Frieri G, Giacomelli R, Pimpo M et al. Mucosal 5-aminosalicylic acid concentration inversely correlates with severity of colonic inflammation in patients with ulcerative colitis. Gut 2000; 47: 410-414

189 Frieri G, Pimpo M, Galletti B et al. Long-term oral plus topical mesalazine in frequently relapsing ulcerative colitis. Digestive and Liver Disease: Official Journal of the Italian Society of Gastroenterology and the Italian Association for the Study of the Liver 2005; 37: 92-96

190 Sutherland L, Macdonald JK. Oral 5-aminosalicylic acid for induction of remission in ulcerative colitis. Cochrane Database of Systematic Reviews (Online) 2006: CD000543

191 Sandborn WJ, Kamm MA, Lichtenstein GR et al. MMX Multi Matrix System mesalazine for the induction of remission in patients with mildto-moderate ulcerative colitis: a combined analysis of two randomized, double-blind, placebo-controlled trials. Aliment Pharmacol Ther 2007; 26: 205-215

192 Lichtenstein GR, Kamm MA, Boddu P et al. Effect of once- or twice-daily MMX mesalamine (SPD476) for the induction of remission of mild to moderately active ulcerative colitis. Clinical Gastroenterology and Hepatology: The Official Clinical Practice Journal of the American Gastroenterological Association 2007; 5: 95-102

193 Kruis W, Kiudelis G, Racz I et al. Once daily versus three times daily mesalazine granules in active ulcerative colitis: a double-blind, double-dummy, randomised, non-inferiority trial. Gut 2009; 58: 233 240

194 Cortot A, Maetz D, Degoutte E et al. Mesalamine foam enema versus mesalamine liquid enema in active left-sided ulcerative colitis. The American Journal of Gastroenterology 2008; 103: 3106-3114

195 Lémann M, Galian A, Rutgeerts P et al. Comparison of budesonide and 5-aminosalicylic acid enemas in active distal ulcerative colitis. Alimentary Pharmacology \& Therapeutics 1995; 9: 557-562

196 Mulder CJ, Fockens P, Meijer JW et al. Beclomethasone dipropionate (3 $\mathrm{mg}$ ) versus 5-aminosalicylic acid $(2 \mathrm{~g})$ versus the combination of both $(3 \mathrm{mg} / 2 \mathrm{~g})$ as retention enemas in active ulcerative proctitis. European Journal of Gastroenterology \& Hepatology 1996; 8: 549-553

197 Lucidarme D, Marteau P, Foucault M et al. Efficacy and tolerance of mesalazine suppositories vs. hydrocortisone foam in proctitis. Alimentary Pharmacology \& Therapeutics 1997; 11: 335

198 Farup PG, Hovde O, Halvorsen FA et al. Mesalazine suppositories versus hydrocortisone foam in patients with distal ulcerative colitis. A comparison of the efficacy and practicality of two topical treatment regimens. Scandinavian Journal of Gastroenterology 1995; 30: 164170

199 Gionchetti P, D’Arienzo A, Rizzello F et al. Topical treatment of distal active ulcerative colitis with beclomethasone dipropionate or mesalamine: a single-blind randomized controlled trial. Journal of Clinical Gastroenterology 2005; 39: 291-297

200 Biancone L, Calabrese E, Petruzziello C et al. Treatment with biologic therapies and the risk of cancer in patients with IBD. Nature Clinical Practice Gastroenterology \& Hepatology 2007; 4: 78-91

201 Manguso F, Balzano A. Meta-analysis: the efficacy of rectal beclomethasone dipropionate vs. 5-aminosalicylic acid in mild to moderate distal ulcerative colitis. Alimentary Pharmacology \& Therapeutics 2007; 26: 21-29

202 Ferry GD, Kirschner BS, Grand RJ et al. Olsalazine versus sulfasalazine in mild to moderate childhood ulcerative colitis: results of the Pediatric Gastroenterology Collaborative Research Group Clinical Trial. Journal of Pediatric Gastroenterology and Nutrition 1993; 17: 32-38

203 Barden L, Lipson A, Pert P et al. Mesalazine in childhood inflammatory bowel disease. Aliment Pharmacol Ther 1989; 3: 597-603
204 Tolia V, Massoud N, Klotz U. Oral 5-aminosalicyclic acid in children with colonic chronic inflammatory bowel disease: clinical and pharmacokinetic experience. J Pediatr Gastroenterol Nutr 1989; 8: 333338

205 Wiersma H, Escher JC, Dilger K et al. Pharmacokinetics of mesalazine pellets in children with inflammatory bowel disease. Inflamm Bowel Dis 2004; 10: 626-631

206 Christensen LA, Fallingborg J, Jacobsen BA et al. Bioavailability of 5aminosalicyclic acid from slow release 5-aminosalicyclic acid drug and sulfasalazine in normal children. Dig Dis Sci 1993; 38: 18311836

207 Cervený P, Bortlík M, Kubena A et al. Nonadherence in inflammatory bowel disease: results of factor analysis. Inflammatory Bowel Diseases 2007; 13: 1244-1249

208 Moshkovska T, Stone M, Baker R et al. Qualitative investigation of patient adherence to 5-aminosalicylic acid therapy in patients with ulcerative colitis. Inflammatory Bowel Diseases 2008; 14: 763-768

209 Kane SV, Accortt NA, Magowan S et al. Predictors of persistence with 5-aminosalicylic acid therapy for ulcerative colitis. Alimentary Pharmacology \& Therapeutics 2009; 29: 855-862

210 Higgins PDR, Rubin DT, Kaulback K et al. Systematic review: impact of non-adherence to 5-aminosalicylic acid products on the frequency and cost of ulcerative colitis flares. Alimentary Pharmacology \& Therapeutics 2009; 29: 247-257

211 Prantera C, Viscido A, Biancone L et al. A new oral delivery system for 5-ASA: preliminary clinical findings for MMx. Inflammatory Bowel Diseases 2005; 11: 421-427

212 Lichtenstein GR, Kamm MA, Sandborn WJ et al. MMX mesalazine for the induction of remission of mild-to-moderately active ulcerative colitis: efficacy and tolerability in specific patient subpopulations. Alimentary Pharmacology \& Therapeutics 2008; 27: 1094-1102

213 Edwards FC, Truelove SC. The Course And Prognosis Of Ulcerative Colitis. Gut 1963; 4: 299-315

214 Turner D, Walsh CM, Steinhart AH et al. Response to corticosteroids in severe ulcerative colitis: a systematic review of the literature and a meta-regression. Clinical Gastroenterology and Hepatology: The Official Clinical Practice Journal of the American Gastroenterological Association 2007; 5: 103-110

215 Baron JH, Connell AM, Kanaghinis TG et al. Out-patient treatment of ulcerative colitis. Comparison between three doses of oral prednisone. British Medical Journal 1962; 2: 441-443

216 Lennard-Jones JE, Longmore AJ, Newell AC et al. An assessment of prednisone, salazopyrin, and topical hydrocortisone hemisuccinate used as out-patient treatment for ulcerative colitis. Gut 1960; 1: 217-222

217 Truelove SC, Witts LJ. Cortisone in ulcerative colitis; preliminary report on a therapeutic trial. British Medical Journal 1954; 2: 375-378

218 Truelove SC, Witts LJ. Cortisone and corticotrophin in ulcerative colitis. British Medical Journal 1959; 1: 387-394

219 Rosenberg W, Ireland A, Jewell DP. High-dose methylprednisolone in the treatment of active ulcerative colitis. Journal of Clinical Gastroenterology 1990; 12: 40-41

220 Turner D, Walsh CM, Benchimol EI et al. Severe paediatric ulcerative colitis: incidence, outcomes and optimal timing for second-line therapy. Gut 2008; 57: 331-338

221 Turner D, Travis SPL, Griffiths A et al. Consensus for Managing Acute Severe Ulcerative Colitis in Children: A Systematic Review and Joint Statement From ECCO, ESPGHAN, and the Porto IBD Working Group of ESPGHAN. Am J Gastroenterol 2011; 106: 574-88

222 Kugathasan S, Dubinsky MC, Keljo D et al. Severe colitis in children. Journal of Pediatric Gastroenterology and Nutrition 2005; 41: 375385

223 Järnerot G, Hertervig E, Friis-Liby I et al. Infliximab as rescue therapy in severe to moderately severe ulcerative colitis: a randomized, placebo-controlled study. Gastroenterology 2005; 128: 1805-1811

224 Lawson MM, Thomas AG, Akobeng AK. Tumour necrosis factor alpha blocking agents for induction of remission in ulcerative colitis. Cochrane Database of Systematic Reviews (Online) 2006; 3: CD005112

225 Ochsenkühn T, Sackmann M, Göke B. Infliximab for acute, not steroidrefractory ulcerative colitis: a randomized pilot study. European Journal of Gastroenterology \& Hepatology 2004; 16: 1167-1171

226 Probert CSJ, Hearing SD, Schreiber S et al. Infliximab in moderately severe glucocorticoid resistant ulcerative colitis: a randomised controlled trial. Gut 2003; 52: 998-1002 
227 Rutgeerts P, Sandborn WJ, Feagan BG et al. Infliximab for induction and maintenance therapy for ulcerative colitis. The New England Journal of Medicine 2005; 353: 2462-2476

228 Shibolet O, Regushevskaya E, Brezis $M$ et al. Cyclosporine A for induction of remission in severe ulcerative colitis. Cochrane Database of Systematic Reviews (Online) 2005: CD004277

229 Lichtiger S, Present DH, Kornbluth A et al. Cyclosporine in severe ulcerative colitis refractory to steroid therapy. The New England Journal of Medicine 1994; 330: 1841-1845

230 D'Haens G, Lemmens L, Geboes K et al. Intravenous cyclosporine versus intravenous corticosteroids as single therapy for severe attacks of ulcerative colitis. Gastroenterology 2001; 120: 1323-1329

231 Baumgart DC, Macdonald JK, Feagan B. Tacrolimus (FK506) for induction of remission in refractory ulcerative colitis. Cochrane Database of Systematic Reviews (Online) 2008: CD007216

232 Baumgart DC, Wiedenmann B, Dignass AU. Rescue therapy with tacrolimus is effective in patients with severe and refractory inflammatory bowel disease. Alimentary Pharmacology \& Therapeutics 2003; 17: 1273-1281

233 Fellermann K, Tanko Z, Herrlinger KR et al. Response of refractory colitis to intravenous or oral tacrolimus (FK506). Inflammatory Bowel Diseases 2002; 8: 317-324

234 Ogata $\mathrm{H}$, Matsui T, Nakamura $M$ et al. A randomised dose finding study of oral tacrolimus (FK506) therapy in refractory ulcerative colitis. Gut 2006; 55: 1255-1262

235 Benazzato L, D'Incà R, Grigoletto $F$ et al. Prognosis of severe attacks in ulcerative colitis: effect of intensive medical treatment. Digestive and Liver Disease: Official Journal of the Italian Society of Gastroenterology and the Italian Association for the Study of the Liver 2004; 36: 461-466

236 Lennard-Jones JE, Ritchie JK, Hilder W et al. Assessment of severity in colitis: a preliminary study. Gut $1975 ; 16: 579-584$

237 Lindgren SC, Flood LM, Kilander AF et al. Early predictors of glucocorticosteroid treatment failure in severe and moderately severe attacks of ulcerative colitis. European Journal of Gastroenterology \& Hepatology 1998; 10: 831-835

238 Travis SP, Farrant JM, Ricketts C et al. Predicting outcome in severe ulcerative colitis. Gut 1996; 38: 905-910

239 Bressler B, Law JK, Al Nahdi Sheraisher N et al. The use of infliximab for treatment of hospitalized patients with acute severe ulcerative colitis. Canadian Journal of Gastroenterology = Journal Canadien De Gastroenterologie 2008; 22: 937-940

240 Campbell S, Travis S, Jewell D. Ciclosporin use in acute ulcerative colitis: a long-term experience. European Journal of Gastroenterology \& Hepatology 2005; 17: 79-84

241 Kohn A, Prantera C, Pera A et al. Anti-tumour necrosis factor alpha (infliximab) in the treatment of severe ulcerative colitis: result of an open study on 13 patients. Digestive and Liver Disease Official: Journal of the Italian Society of Gastroenterology and the Italian Association for the Study of the Liver 2002; 34: 626-630

242 Sands BE, Tremaine WJ, Sandborn WJ et al. Infliximab in the treatment of severe, steroid-refractory ulcerative colitis: a pilot study. Inflammatory Bowel Diseases 2001; 7: 83-88

243 Cacheux W, Seksik P, Lemann $M$ et al. Predictive factors of response to cyclosporine in steroid-refractory ulcerative colitis. The American Journal of Gastroenterology 2008; 103: 637-642

244 Cohen RD, Brodsky AL, Hanauer SB. A comparison of the quality of life in patients with severe ulcerative colitis after total colectomy versus medical treatment with intravenous cyclosporin. Inflammatory Bowel Diseases 1999; 5: 1-10

245 Van Assche G, Dalle I, Noman M et al. A pilot study on the use of the humanized anti-interleukin-2 receptor antibody daclizumab in active ulcerative colitis. The American Journal of Gastroenterology 2003; 98: 369-376

246 Baumgart DC, Pintoffl JP, Sturm A et al. Tacrolimus is safe and effective in patients with severe steroid-refractory or steroid-dependent inflammatory bowel disease-a long-term follow-up. The American Journal of Gastroenterology 2006; 101: 1048-1056

247 Pascu M, Müller AR, Wiedenmann B et al. Rescue therapy with tacrolimus in a patient with toxic megacolon. International Journal of Colorectal Disease 2003; 18: 271-275

248 Ardizzone S, Maconi G, Russo A et al. Randomised controlled trial of azathioprine and 5-aminosalicylic acid for treatment of steroid dependent ulcerative colitis. Gut 2006; 55: 47-53
249 Jewell DP, Truelove SC. Azathioprine in ulcerative colitis: final report on controlled therapeutic trial. British Medical Journal 1974; 4: 627-630

250 Rosenberg JL, Wall AJ, Levin B et al. A controlled trial of azathioprine in the management of chronic ulcerative colitis. Gastroenterology 1975; 69: 96-99

251 Derijks LJJ, Gilissen LPL, Hooymans PM et al. Review article: thiopurines in inflammatory bowel disease. Alimentary Pharmacology \& Therapeutics 2006; 24: 715-729

252 Dubinsky MC. Azathioprine, 6-mercaptopurine in inflammatory bowel disease: pharmacology, efficacy, and safety. Clinical Gastroenterology and Hepatology: The Official Clinical Practice Journal of the American Gastroenterological Association 2004; 2: 731-743

253 Gisbert JP, Linares PM, McNicholl AG et al. Meta-analysis: the efficacy of azathioprine and mercaptopurine in ulcerative colitis. Aliment Pharmacol Ther 2009; 30: 126-137

254 Adler DJ, Korelitz BI. The therapeutic efficacy of 6-mercaptopurine in refractory ulcerative colitis. The American Journal of Gastroenterology 1990; 85: 717-722

255 Ardizzone S, Molteni $P$, Imbesi $V$ et al. Azathioprine in steroid-resistant and steroid-dependent ulcerative colitis. Journal of Clinical Gastroenterology 1997; 25: 330-333

256 Fraser AG, Orchard TR, Robinson EM et al. Long-term risk of malignancy after treatment of inflammatory bowel disease with azathioprine. Alimentary Pharmacology \& Therapeutics 2002; 16: 1225-1232

257 Paoluzi OA, Pica R, Marcheggiano A et al. Azathioprine or methotrexate in the treatment of patients with steroid-dependent or steroid-resistant ulcerative colitis: results of an open-label study on efficacy and tolerability in inducing and maintaining remission. Alimentary Pharmacology \& Therapeutics 2002; 16: 1751-1759

258 Steinhart AH, Baker JP, Brzezinski A et al. Azathioprine therapy in chronic ulcerative colitis. Journal of Clinical Gastroenterology 1990; 12: $271-275$

259 Andrews JM, Travis SPL, Gibson PR et al. Systematic review: does concurrent therapy with 5-ASA and immunomodulators in inflammatory bowel disease improve outcomes? Alimentary Pharmacology \& Therapeutics 2009; 29: 459-469

260 Hanauer SB, Sandborn WJ, Dallaire C et al. Delayed-release oral mesalamine $4.8 \mathrm{~g} /$ day ( $800 \mathrm{mg}$ tablets) compared to $2.4 \mathrm{~g} /$ day $(400 \mathrm{mg}$ tablets) for the treatment of mildly to moderately active ulcerative colitis: The ASCEND I trial. Canadian Journal of Gastroenterology = Journal Canadien De Gastroenterologie 2007; 21: 827-834

261 Gan SI, Beck PL. A new look at toxic megacolon: an update and review of incidence, etiology, pathogenesis, and management. The American Journal of Gastroenterology 2003; 98: 2363-2371

262 Sheth SG, LaMont JT. Toxic megacolon. Lancet 1998; 351: 509-513

263 Longo WE, Virgo KS, Bahadursingh AN et al. Patterns of disease and surgical treatment among United States veterans more than 50 years of age with ulcerative colitis. American Journal of Surgery 2003; 186 : $514-518$

264 Kopf A, Janson W, Stein C. Opioid therapy in chronic non-malignant pain. Der Anaesthesist 2003; 52: 103-114

265 Gerson LB, Triadafilopoulos G. Palliative care in inflammatory bowel disease: an evidence-based approach. Inflammatory Bowel Diseases 2000; 6: 228-243

266 Arundel C, Lewis JH. Drug-induced liver disease in 2006. Current Opinion in Gastroenterology 2007; 23: 244-254

267 Edwards JT, Radford-Smith GL, Florin TH. Chronic narcotic use in inflammatory bowel disease patients: prevalence and clinical characteristics. Journal of Gastroenterology and Hepatology 2001; 16: 12351238

268 Kaplan MA, Korelitz BI. Narcotic dependence in inflammatory bowel disease. Journal of Clinical Gastroenterology 1988; 10: 275-278

269 White M, Shah N, Lindley K et al. Pain management in fulminating ulcerative colitis. Paediatric Anaesthesia 2006; 16: 1148-1152

$270 \mathrm{BebbJR}$, Scott BB. How effective are the usual treatments for ulcerative colitis? Alimentary Pharmacology \& Therapeutics 2004; 20: 143-149

271 Sandborn WJ, Hanauer SB. Systematic review: the pharmacokinetic profiles of oral mesalazine formulations and mesalazine pro-drugs used in the management of ulcerative colitis. Alimentary Pharmacology \& Therapeutics 2003; 17: 29-42

$272 \mathrm{Klotz}$ U. Colonic targeting of aminosalicylates for the treatment of ulcerative colitis. Digestive and Liver Disease: Official Journal of the Italian Society of Gastroenterology and the Italian Association for the Study of the Liver 2005; 37 : 381-388 
273 Van Staa TP, Travis S, Leufkens HGM et al. 5-aminosalicylic acids and the risk of renal disease: a large British epidemiologic study. Gastroenterology 2004; 126: 1733-1739

274 Diak P, Siegel J, La Grenade L et al. Malignancy in children and tumor necrosis factor-alpha blockers: Forty-eight cases reported to the food and drug administration. Arthritis and Rheumatism 2010; 62: 2517 2524

275 Horneff G, Hospach T, Dannecker $G$ et al. Updated statement by the German Society for Pediatric and Adolescent Rheumatology (GKJR) on the FDA's report regarding malignancies in anti-TNF-treated patients from Aug. 4, 2009. Zeitschrift Fur Rheumatologie 2010; 69: $561-567$

276 Jones JL, Loftus EV. Lymphoma risk in inflammatory bowel disease: is it the disease or its treatment? Inflammatory Bowel Diseases 2007; 13: 1299-1307

277 Reinshagen M, Schütz E, Armstrong VW et al. 6-thioguanine nucleotide-adapted azathioprine therapy does not lead to higher remission rates than standard therapy in chronic active crohn disease: results from a randomized, controlled, open trial. Clinical Chemistry 2007; 53: $1306-1314$

278 Gonvers JJ, Juillerat P, Mottet C et al. Maintenance of medically induced remission of Crohn's disease. Digestion 2007; 76: 116-129

279 Lémann M, Mary JY, Colombel JF et al. A randomized, double-blind, controlled withdrawal trial in Crohn's disease patients in long-term remission on azathioprine. Gastroenterology 2005; 128: 1812-1818

280 Vilien M, Dahlerup JF, Munck LK et al. Randomized controlled azathioprine withdrawal after more than two years treatment in Crohn's disease: increased relapse rate the following year. Alimentary Pharmacology \& Therapeutics 2004; 19: 1147-1152

281 Colombel JF, Ferrari N, Debuysere H et al. Genotypic analysis of thiopurine S-methyltransferase in patients with Crohn's disease and severe myelosuppression during azathioprine therapy. Gastroenterology 2000; 118: 1025-1030

282 Schedel J, Gödde A, Schütz E et al. Impact of thiopurine methyltransferase activity and 6-thioguanine nucleotide concentrations in patients with chronic inflammatory diseases. Annals of the New York Academy of Sciences 2006; 1069: 477-491

283 Moskovitz DN, Van Assche G, Maenhout B et al. Incidence of colectomy during long-term follow-up after cyclosporine-induced remission of severe ulcerative colitis. Clinical Gastroenterology and Hepatology: The Official Clinical Practice Journal of the American Gastroenterological Association 2006; 4: 760-765

284 Penfornis A, Kury-Paulin S. Immunosuppressive drug-induced diabetes. Diabetes \& Metabolism 2006; 32: 539-546

285 Oren $R$, Arber N, Odes $S$ et al. Methotrexate in chronic active ulcerative colitis: a double-blind, randomized, Israeli multicenter trial. Gastroenterology 1996; 110: 1416-1421

286 Maté-Jiménez J, Hermida C, Cantero-Perona J et al. 6-mercaptopurine or methotrexate added to prednisone induces and maintains remission in steroid-dependent inflammatory bowel disease. European Journal of Gastroenterology \& Hepatology 2000; 12: 1227-1233

287 Roth LS, Chande N, Ponich T et al. Predictors of disease severity in ulcerative colitis patients from Southwestern Ontario. World J Gastroenterol 2010; 16: 232-236

288 Vucelic B. Inflammatory bowel diseases: controversies in the use of diagnostic procedures. Dig Dis 2009; 27: 269-277

289 Kane S, Lu F, Kornbluth A et al. Controversies in mucosal healing in ulcerative colitis. Inflamm Bowel Dis 2009; 15: 796-800

290 Brain 0, Travis SP. Therapy of ulcerative colitis: state of the art. Curr Opin Gastroenterol 2008; 24: 469-474

291 Rutgeerts P, Vermeire S, Van Assche G. Mucosal healing in inflammatory bowel disease: impossible ideal or therapeutic target? Gut 2007; 56: 453-455

292 Rahimi R, Nikfar S, Rezaie A et al. Comparison of mesalazine and balsalazide in induction and maintenance of remission in patients with ulcerative colitis: a meta-analysis. Dig Dis Sci 2009; 54: 712-721

293 Sutherland L, Macdonald JK. Oral 5-aminosalicylic acid for maintenance of remission in ulcerative colitis. Cochrane Database Syst Rev 2006: CD000544

294 Cohen RD, Woseth DM, Thisted RA et al. A meta-analysis and overview of the literature on treatment options for left-sided ulcerative colitis and ulcerative proctitis. Am J Gastroenterol 2000; 95: 1263-1276

295 Kornbluth AA, Salomon P, Sacks HS et al. Meta-analysis of the effectiveness of current drug therapy of ulcerative colitis. J Clin Gastroenterol 1993; 16: 215-218
296 Nikfar S, Rahimi R, Rezaie A et al. A meta-analysis of the efficacy of sulfasalazine in comparison with 5-aminosalicylates in the induction of improvement and maintenance of remission in patients with ulcerative colitis. Dig Dis Sci 2009; 54: 1157-1170

297 Sandborn WJ. Treatment of ulcerative colitis with oral mesalamine: advances in drug formulation, efficacy expectations and dose response, compliance, and chemoprevention. Rev Gastroenterol Disord 2006; 6: 97-105

298 Hanauer SB. Review article: high-dose aminosalicylates to induce and maintain remissions in ulcerative colitis. Aliment Pharmacol Ther 2006; 24 (Suppl 3): 37-40

299 Marshall JK, Irvine EJ. Rectal corticosteroids versus alternative treatments in ulcerative colitis: a meta-analysis. Gut 1997; 40: 775-781

300 d'Albasio G, Pacini F, Camarri E et al. Combined therapy with 5-aminosalicylic acid tablets and enemas for maintaining remission in ulcerative colitis: a randomized double-blind study. Am J Gastroenterol 1997; 92: 1143-1147

301 Piodi LP, Ulivieri FM, Cermesoni L et al. Long-term intermittent treatment with low-dose 5-aminosalicylic enemas is efficacious for remission maintenance in ulcerative colitis. Scand J Gastroenterol 2004; 39: $154-157$

302 Fockens P, Mulder CJ, Tytgat GN et al. Comparison of the efficacy and safety of 1.5 compared with $3.0 \mathrm{~g}$ oral slow-release mesalazine (Pentasa) in the maintenance treatment of ulcerative colitis. Dutch Pentasa Study Group. Eur J Gastroenterol Hepatol 1995; 7: 1025-1030

303 Kamm MA, Sandborn WJ, Gassull M et al. Once-daily, high-concentration MMX mesalamine in active ulcerative colitis. Gastroenterology 2007; 132: 66-75; quiz 432 - 433

304 Kiilerich S, Ladefoged K, Rannem T et al. Prophylactic effects of olsalazine $\mathrm{v}$ sulphasalazine during 12 months maintenance treatment of ulcerative colitis. The Danish Olsalazine Study Group. Gut 1992; 33: 252-255

305 Azad Khan AK, Howes DT, Piris J et al. Optimum dose of sulphasalazine for maintenance treatment in ulcerative colitis. Gut 1980; 21 : 232 240

306 Biddle WL, Greenberger NJ, Swan JT et al. 5-Aminosalicylic acid enemas: effective agent in maintaining remission in left-sided ulcerative colitis. Gastroenterology 1988; 94: 1075-1079

307 Mantzaris GJ, Hatzis A, Petraki K et al. Intermittent therapy with highdose 5-aminosalicylic acid enemas maintains remission in ulcerative proctitis and proctosigmoiditis. Dis Colon Rectum 1994; 37: 58-62

308 d'Albasio G, Trallori G, Ghetti A et al. Intermittent therapy with highdose 5-aminosalicylic acid enemas for maintaining remission in ulcerative proctosigmoiditis. Dis Colon Rectum 1990; 33: 394-397

309 d'Albasio G, Paoluzi P, Campieri M et al. Maintenance treatment of ulcerative proctitis with mesalazine suppositories: a double-blind placebo-controlled trial. The Italian IBD Study Group. Am J Gastroenterol 1998; 93: 799-803

310 Marteau P, Crand J, Foucault M et al. Use of mesalazine slow release suppositories $1 \mathrm{~g}$ three times per week to maintain remission of ulcerative proctitis: a randomised double blind placebo controlled multicentre study. Gut 1998; 42: 195-199

$311 \mathrm{Kamm}$ MA, Lichtenstein GR, Sandborn WJ et al. Randomised trial of once- or twice-daily MMX mesalazine for maintenance of remission in ulcerative colitis. Gut 2008; 57: 893-902

312 Orchard T, Probert CS, Keshav S. Review article: maintenance therapy in patients with ulcerative colitis. Aliment Pharmacol Ther 2006; 24 (Suppl 1): 17-22

313 Bickston SJ, Cominelli F. Optimal dosing of 5-aminosalicylic acid: 5 decades of choosing between politicians. Clin Gastroenterol Hepatol 2003; $1: 3-4$

314 Dignass AU, Bokemeyer B, Adamek $H$ et al. Mesalamine once daily is more effective than twice daily in patients with quiescent ulcerative colitis. Clin Gastroenterol Hepatol 2009; 7: 762-769

315 Prantera C, Kohn A, Campieri $M$ et al. Clinical trial: ulcerative colitis maintenance treatment with 5-ASA: a 1-year, randomized multicentre study comparing MMX with Asacol. Aliment Pharmacol Ther 2009; 30: 908-918

316 Ardizzone S, Porro GB. How long is it advisable to prolong maintenance treatment of patients with ulcerative colitis? Inflamm Bowel Dis 2008; 14 (Suppl 2): S238-S239

317 Ardizzone S, Petrillo $M$, Imbesi $V$ et al. Is maintenance therapy always necessary for patients with ulcerative colitis in remission? Aliment Pharmacol Ther 1999; 13: 373-379 
318 Patel H, Barr A, Jeejeebhoy KN. Renal effects of long-term treatment with 5-aminosalicylic acid. Can J Gastroenterol 2009; 23: 170-176

319 d'Albasio G, Trallori G, Gavazzi 0 et al. Combined therapy with 5-aminosalicylic tablets and enemas for maintaining remission in ulcerative colitis. Ital J Gastroenterol 1991; 23: 12-14

320 Timmer A, McDonald JW, Macdonald JK. Azathioprine and 6-mercaptopurine for maintenance of remission in ulcerative colitis. Cochrane Database Syst Rev 2007: CD000478

321 Russo EA, Harris AW, Campbell S et al. Experience of maintenance infliximab therapy for refractory ulcerative colitis from six centres in England. Aliment Pharmacol Ther 2009; 29: 308-314

322 Caviglia R, Ribolsi M, Rizzi M et al. Maintenance of remission with infliximab in inflammatory bowel disease: efficacy and safety longterm follow-up. World J Gastroenterol 2007; 13: 5238-5244

323 Carbonnel F, Boruchowicz A, Duclos B et al. Intravenous cyclosporine in attacks of ulcerative colitis: short-term and long-term responses. Dig Dis Sci 1996; 41: 2471-2476

324 Campbell S, Ghosh S. Combination immunomodulatory therapy with cyclosporine and azathioprine in corticosteroid-resistant severe ulcerative colitis: the Edinburgh experience of outcome. Dig Liver Dis 2003; 35: 546-551

325 Actis GC, Lagget M, Rizzetto $M$ et al. Long-term efficacy of oral microemulsion cyclosporin for refractory ulcerative colitis. Minerva Med 2004; 95: 65-70

326 Rolny P, Sadik R. Longer term outcome of steroid refractory ulcerative colitis treated with intravenous cyclosporine without subsequent oral cyclosporine maintenance therapy. Int J Colorectal Dis 2002; 17: 67-69

327 Cohen RD, Stein R, Hanauer SB. Intravenous cyclosporin in ulcerative colitis: a five-year experience. Am J Gastroenterol 1999; 94: 15871592

328 Kruis W, Schreiber S, Theuer D et al. Low dose balsalazide (1.5 g twice daily) and mesalazine ( $0.5 \mathrm{~g}$ three times daily) maintained remission of ulcerative colitis but high dose balsalazide ( $3.0 \mathrm{~g}$ twice daily) was superior in preventing relapses. Gut 2001; 49: 783-789

329 Actis GC, Fadda M, Pellicano R et al. The 17-year single-center experience with the use of azathioprine to maintain remission in ulcerative colitis. Biomed Pharmacother 2009; 63: 362-365

330 Mantzaris GJ, Sfakianakis M, Archavlis E et al. A prospective randomized observer-blind 2-year trial of azathioprine monotherapy versus azathioprine and olsalazine for the maintenance of remission of steroid-dependent ulcerative colitis. Am J Gastroenterol 2004; 99: $1122-1128$

331 Domenech E, Garcia-Planella E, Bernal I et al. Azathioprine without oral ciclosporin in the long-term maintenance of remission induced by intravenous ciclosporin in severe, steroid-refractory ulcerative colitis. Aliment Pharmacol Ther 2002; 16: 2061-2065

332 Cassinotti A, Actis GC, Duca P et al. Maintenance treatment with azathioprine in ulcerative colitis: outcome and predictive factors after drug withdrawal. Am J Gastroenterol 2009; 104: 2760-2767

333 Oussalah A, Danese S, Peyrin-Biroulet L. Efficacy of TNF Antagonists Beyond One Year in Adult and Pediatric Inflammatory Bowel Diseases: A Systematic Review. Curr Drug Targets 2009; 11: 156-175

334 El-Matary W, Vandermeer B, Griffiths AM. Methotrexate for maintenance of remission in ulcerative colitis. Cochrane Database Syst Rev 2009: CD007560

335 Herrlinger KR, Fellermann K, Stange EF. Tacrolimus - finally! Gut 2006; 55: 1224-1225

336 Lindgren S, Lofberg R, Bergholm L et al. Effect of budesonide enema on remission and relapse rate in distal ulcerative colitis and proctitis. Scand J Gastroenterol 2002; 37: 705-710

337 Meyers S, Lerer PK, Feuer EJ et al. Predicting the outcome of corticoid therapy for acute ulcerative colitis. Results of a prospective, randomized, double-blind clinical trial. J Clin Gastroenterol 1987; 9: 50-54

338 Kruis W, Fric P, Pokrotnieks J et al. Maintaining remission of ulcerative colitis with the probiotic Escherichia coli Nissle 1917 is as effective as with standard mesalazine. Gut 2004; 53: 1617-1623

339 Kruis W, Schutz E, Fric $P$ et al. Double-blind comparison of an oral Escherichia coli preparation and mesalazine in maintaining remission of ulcerative colitis. Aliment Pharmacol Ther 1997; 11: 853-858

340 Rembacken BJ, Snelling AM, Hawkey PM et al. Non-pathogenic Escherichia coli versus mesalazine for the treatment of ulcerative colitis: a randomised trial. Lancet 1999; 354: 635-639

341 Henker J, Muller S, Laass MW et al. Probiotic Escherichia coli Nissle 1917 (EcN) for successful remission maintenance of ulcerative colitis in children and adolescents: an open-label pilot study. Z Gastroenterol 2008; 46: 874-875

342 Miele E, Pascarella F, Giannetti E et al. Effect of a probiotic preparation (VSL\#3) on induction and maintenance of remission in children with ulcerative colitis. Am J Gastroenterol 2009; 104: 437-443

343 Solomons NW. Malnutrition and infection: an update. The British Journal of Nutrition 2007; 98 (Suppl 1): S5-S10

344 Chandra RK. Nutrition, immunity and infection: from basic knowledge of dietary manipulation of immune responses to practical application of ameliorating suffering and improving survival. Proceedings of the National Academy of Sciences of the United States of America 1996; 93: 14304-14307

345 Guerrant RL, Lima AA, Davidson F. Micronutrients and infection: interactions and implications with enteric and other infections and future priorities. The Journal of Infectious Diseases 2000; 182 (Suppl 1): S134-S138

346 Castle SC. Clinical relevance of age-related immune dysfunction. Clinical Infectious Diseases: An Official Publication of the Infectious Diseases Society of America 2000; 31: 578-585

347 Page MJ, Poritz LS, Kunselman SJ et al. Factors affecting surgical risk in elderly patients with inflammatory bowel disease. Journal of Gastrointestinal Surgery: Official Journal of the Society for Surgery of the Alimentary Tract 2002; 6: 606-613

348 Crum NF, Lederman ER, Wallace MR. Infections associated with tumor necrosis factor-alpha antagonists. Medicine 2005; 84: 291-302

349 Gaemperli A, Hauser T, Speck R. Risk of infection during treatment with tumor necrosis factor-alpha inhibitors. Zeitschrift Fur Rheumatologie 2006; 65: 24-28, 30 - 31

350 Gupta G, Lautenbach E, Lewis JD. Incidence and risk factors for herpes zoster among patients with inflammatory bowel disease. Clinical Gastroenterology and Hepatology: The Official Clinical Practice Journal of the American Gastroenterological Association 2006; 4: 1483 1490

351 Viget N, Vernier-Massouille G, Salmon-Ceron D et al. Opportunistic infections in patients with inflammatory bowel disease: prevention and diagnosis. Gut 2008; 57: 549-558

352 Tsiodras S, Samonis G, Boumpas DT et al. Fungal infections complicating tumor necrosis factor alpha blockade therapy. Mayo Clinic Proceedings Mayo Clinic 2008; 83: 181-194

353 Hagel S, Bruns T, Kantowski $M$ et al. Cholestatic hepatitis, acute acalculous cholecystitis, and hemolytic anemia: primary Epstein-Barr virus infection under azathioprine. Inflammatory Bowel Diseases 2009; 15: 1613-1616

354 Stuck AE, Minder CE, Frey FJ. Risk of infectious complications in patients taking glucocorticosteroids. Reviews of Infectious Diseases 1989; 11: 954-963

355 Jick SS, Lieberman ES, Rahman MU et al. Glucocorticoid use, other associated factors, and the risk of tuberculosis. Arthritis and Rheumatism 2006; 55: 19-26

356 Yang $Z, W u Q$ Wu K et al. Meta-analysis: pre-operative infliximab treatment and short-term post-operative complications in patients with ulcerative colitis. Alimentary Pharmacology \& Therapeutics 2010; 31: 486-492

357 Schneeweiss S, Setoguchi S, Weinblatt ME et al. Anti-tumor necrosis factor alpha therapy and the risk of serious bacterial infections in elderly patients with rheumatoid arthritis. Arthritis and Rheumatism 2007; 56: 1754-1764

358 Fidder H, Schnitzler F, Ferrante $M$ et al. Long-term safety of infliximab for the treatment of inflammatory bowel disease: a single-centre cohort study. Gut 2009; 58: 501-508

359 Lichtenstein GR, Feagan BG, Cohen RD et al. Serious infections and mortality in association with therapies for Crohn's disease: TREAT registry. Clinical Gastroenterology and Hepatology: The Official Clinical Practice Journal of the American Gastroenterological Association 2006; 4: 621-630

360 Keane J, Gershon S, Wise RP et al. Tuberculosis associated with infliximab, a tumor necrosis factor alpha-neutralizing agent. The New England Journal of Medicine 2001; 345: 1098-1104

361 Bakleh M, Tleyjeh I, Matteson EL et al. Infectious complications of tumor necrosis factor-alpha antagonists. International Journal of Dermatology 2005; 44: 443-448

362 Dederichs F, Pinciu F, Gerhard $H$ et al. Listeria meningitis in a patient with Crohn's disease - a seldom, but clinically relevant adverse event of therapy with infliximab. Zeitschrift Für Gastroenterologie 2006; 44: 657-660 
363 Tiede I, Fritz G, Strand S et al. CD28-dependent Rac1 activation is the molecular target of azathioprine in primary human CD 4 + T lymphocytes. The Journal of Clinical Investigation 2003; 111: 1133-1145

364 Furst MB, Stromberg BV, Blatchford GJ et al. Colonic anastomoses: bursting strength after corticosteroid treatment. Diseases of the Colon and Rectum 1994; 37: 12-15

365 Markel TA, Lou DC, Pfefferkorn $M$ et al. Steroids and poor nutrition are associated with infectious wound complications in children undergoing first stage procedures for ulcerative colitis. Surgery 2008; 144 : 540-545; discussion 545 - 547

366 Mahadevan U, Loftus EV, Tremaine WJ et al. Azathioprine or 6-mercaptopurine before colectomy for ulcerative colitis is not associated with increased postoperative complications. Inflammatory Bowel Diseases 2002; 8: 311-316

367 Subramanian V, Saxena S, Kang JY et al. Preoperative steroid use and risk of postoperative complications in patients with inflammatory bowel disease undergoing abdominal surgery. The American Journal of Gastroenterology 2008; 103: 2373-2381

368 Subramanian V, Pollok RCG, Kang JY et al. Systematic review of postoperative complications in patients with inflammatory bowel disease treated with immunomodulators. Br J Surg 2006; 93: 793-799

369 Colombel JF, Loftus EV, Tremaine WJ et al. Early postoperative complications are not increased in patients with Crohn's disease treated perioperatively with infliximab or immunosuppressive therapy. The American Journal of Gastroenterology 2004; 99: 878-883

370 Schluender SJ, Ippoliti A, Dubinsky M et al. Does infliximab influence surgical morbidity of ileal pouch-anal anastomosis in patients with ulcerative colitis? Dis Colon Rectum 2007; 50: 1747-1753

371 Selvasekar CR, Cima RR, Larson DW et al. Effect of infliximab on shortterm complications in patients undergoing operation for chronic ulcerative colitis. Journal of the American College of Surgeons 2007; 204: 956-962; discussion 962 - 953

372 Appau KA, Fazio VW, Shen B et al. Use of infliximab within 3 months of ileocolonic resection is associated with adverse postoperative outcomes in Crohn's patients. Journal of Gastrointestinal Surgery: Official Journal of the Society for Surgery of the Alimentary Tract 2008; 12 : 1738-1744

373 Kunitake H, Hodin R, Shellito PC et al. Perioperative treatment with infliximab in patients with Crohn's disease and ulcerative colitis is not associated with an increased rate of postoperative complications. Journal of Gastrointestinal Surgery: Official Journal of the Society for Surgery of the Alimentary Tract 2008; 12: 1730-1736; discussion $1736-1737$

374 Mor IJ, Vogel JD, da Luz Moreira A et al. Infliximab in ulcerative colitis is associated with an increased risk of postoperative complications after restorative proctocolectomy. Diseases of the Colon and Rectum 2008; 51: 1202-1207; discussion 1207 - 1210

375 Indar AA, Young-Fadok TM, Heppell J et al. Effect of perioperative immunosuppressive medication on early outcome in Crohn's disease patients. World J Surg 2009; 33: 1049-1052

376 Ferrante M, D'Hoore A, Vermeire S et al. Corticosteroids but not infliximab increase short-term postoperative infectious complications in patients with ulcerative colitis. Inflamm Bowel Dis 2009; 15: 10621070

377 Blum HE, Berg T, Tillmann HL et al. Procedures for problematic situations with hepatitis B. Zeitschrift Für Gastroenterologie 2004; 42: 692-697

378 Domm S, Cinatl J, Mrowietz U. The impact of treatment with tumour necrosis factor-alpha antagonists on the course of chronic viral infections: a review of the literature. The British Journal of Dermatology 2008; 159: 1217-1228

379 Mindikoglu AL, Regev A, Schiff ER. Hepatitis B virus reactivation after cytotoxic chemotherapy: the disease and its prevention. Clinical Gastroenterology and Hepatology: The Official Clinical Practice Journal of the American Gastroenterological Association 2006; 4: 1076-1081

380 Esteve $M$, Saro C, González-Huix F et al. Chronic hepatitis B reactivation following infliximab therapy in Crohn's disease patients: need for primary prophylaxis. Gut 2004; 53: 1363-1365

381 Lau GKK. Hepatitis B reactivation after chemotherapy: two decades of clinical research. Hepatology International 2008; 2: 152-162

382 European Association For The Study of The LEASL. Clinical Practice Guidelines: management of chronic hepatitis B. Journal of Hepatology 2009; 50: 227-242

383 Schoepfer AM, Flogerzi B, Fallegger S et al. Comparison of interferongamma release assay versus tuberculin skin test for tuberculosis screening in inflammatory bowel disease. The American Journal of Gastroenterology 2008; 103: 2799-2806

384 Diel R, Forßbohm M, Loytved $G$ et al. Empfehlungen für die Umgebungsuntersuchungen bei Tuberkulose. Pneumologie 2007; 61: 440-455

385 Aslanidis S, Vassiliadis T, Pyrpasopoulou A et al. Inhibition of TNFalpha does not induce viral reactivation in patients with chronic hepatitis $C$ infection: two cases. Clinical Rheumatology 2007; 26: 261-264

386 Campbell S, Ghosh S. Infliximab therapy for Crohn's disease in the presence of chronic hepatitis C infection. European Journal of Gastroenterology \& Hepatology 2001; 13: 191-192

387 Nissen MJ, Fontanges E, Allam Y et al. Rheumatological manifestations of hepatitis C: incidence in a rheumatology and non-rheumatology setting and the effect of methotrexate and interferon. Rheumatology 2005; 44: 1016-1020

388 Vivarelli M, Burra P, La Barba G et al. Influence of steroids on HCV recurrence after liver transplantation: A prospective study. Journal of Hepatology 2007; 47: 793-798

389 Stangl JR, Carroll KL, Illichmann $M$ et al. Effect of antimetabolite immunosuppressants on Flaviviridae, including hepatitis $C$ virus. Transplantation 2004; 77: 562-567

390 Scherzer TM, Staufer K, Novacek G et al. Efficacy and safety of antiviral therapy in patients with Crohn's disease and chronic hepatitis C. Alimentary Pharmacology \& Therapeutics 2008; 15: 742 - 748

391 Hoffmann $H$, Loytved $G$, Bodmer T. Interferon-gamma release assays in tuberculosis diagnostics. Der Internist 2007; 48: 497-498, 500 - 506

392 Ehlers $S$. Why does tumor necrosis factor targeted therapy reactivate tuberculosis? The Journal of Rheumatology Supplement 2005; 74: 35-39

393 Wallis RS, Broder MS, Wong JY et al. Granulomatous infectious diseases associated with tumor necrosis factor antagonists. Clinical Infectious Diseases: An Official Publication of the Infectious Diseases Society of America 2004; 38: 1261-1265

394 Tubach F, Salmon D, Ravaud P et al. Risk of tuberculosis is higher with anti-tumor necrosis factor monoclonal antibody therapy than with soluble tumor necrosis factor receptor therapy: The three-year prospective French Research Axed on Tolerance of Biotherapies registry. Arthritis and Rheumatism 2009; 60: 1884-1894

395 Gómez-Reino JJ, Carmona L, Angel Descalzo M. Risk of tuberculosis in patients treated with tumor necrosis factor antagonists due to incomplete prevention of reactivation of latent infection. Arthritis and Rheumatism 2007; 57: 756-761

396 Seong SS, Choi CB, Woo JH et al. Incidence of tuberculosis in Korean patients with rheumatoid arthritis (RA): effects of RA itself and of tumor necrosis factor blockers. The Journal of Rheumatology 2007; 34: 706711

397 Aberra FN, Stettler N, Brensinger C et al. Risk for active tuberculosis in inflammatory bowel disease patients. Clinical Gastroenterology and Hepatology: The Official Clinical Practice Journal of the American Gastroenterological Association 2007; 5: 1070-1075

398 Schiff MH, Burmester GR, Kent JD et al. Safety analyses of adalimumab (HUMIRA) in global clinical trials and US postmarketing surveillance of patients with rheumatoid arthritis. Annals of the Rheumatic Diseases 2006; 65: 889-894

399 Wallis RS. Mathematical modeling of the cause of tuberculosis during tumor necrosis factor blockade. Arthritis and Rheumatism 2008; 58: 947-952

400 Carmona L, Gómez-Reino JJ, Rodríguez-Valverde V et al. Effectiveness of recommendations to prevent reactivation of latent tuberculosis infection in patients treated with tumor necrosis factor antagonists. $\mathrm{Ar}$ thritis and Rheumatism 2005; 52: 1766-1772

401 Sichletidis L, Settas L, Spyratos D et al. Tuberculosis in patients receiving anti-TNF agents despite chemoprophylaxis. The International Journal of Tuberculosis and Lung Disease: The Official Journal of the International Union Against Tuberculosis and Lung Disease 2006; 10: 1127-1132

402 Magro F, Pereira P, Veloso FT et al. Unusual presentation of tuberculosis after infliximab therapy. Inflammatory Bowel Diseases 2005; 11: 82-84

403 Keane J, Bresnihan B. Tuberculosis reactivation during immunosuppressive therapy in rheumatic diseases: diagnostic and therapeutic strategies. Current Opinion in Rheumatology 2008; 20: 443-449

404 Sibartie V, Kirwan WO, O'Mahony S et al. Intestinal tuberculosis mimicking Crohn's disease: lessons relearned in a new era. European Journal of Gastroenterology \& Hepatology 2007; 19: 347-349 
405 Paul Ehrlich Institut. Empfehlungen zur Diagnose und Prävention der latenten Tuberkulose bei Patienten vor Beginn der Behandlung mit Remicade (Infliximab). Februar 2002

406 Diel R, Hauer B, Loddenkemper $R$ et al. Recommendations for tuberculosis screening before initiation of TNF-alpha-inhibitor treatment in rheumatic diseases. Pneumologie 2009; 63: 329-334

407 Efthimiou P, Sood S. QuantiFERON TB Gold Test: the new standard for screening of latent tuberculosis in patients with rheumatoid arthritis? Annals of the Rheumatic Diseases 2007; 66: 276

408 Sellam J, Hamdi H, Roy C et al. Comparison of in vitro-specific blood tests with tuberculin skin test for diagnosis of latent tuberculosis before anti-TNF therapy. Annals of the Rheumatic Diseases 2007; 66: 1610-1615

409 Raval A, Akhavan-Toyserkani G, Brinker A et al. Brief communication: characteristics of spontaneous cases of tuberculosis associated with infliximab. Annals of Internal Medicine 2007; 147: 699-702

410 Vassilopoulos D, Stamoulis N, Hadziyannis E et al. Usefulness of enzyme-linked immunospot assay (Elispot) compared to tuberculin skin testing for latent tuberculosis screening in rheumatic patients scheduled for anti-tumor necrosis factor treatment. The Journal of Rheumatology 2008; 35: 1271-1276

411 Beglinger C, Dudler J, Mottet C et al. Screening for tuberculosis infection before the initiation of an anti-TNF-alpha therapy. Swiss Medical Weekly: Official Journal of the Swiss Society of Infectious Diseases, the Swiss Society of Internal Medicine, the Swiss Society of Pneumology 2007; 137: 620-622

412 Mow WS, Abreu-Martin MT, Papadakis KA et al. High incidence of anergy in inflammatory bowel disease patients limits the usefulness of PPD screening before infliximab therapy. Clinical Gastroenterology and Hepatology: The Official Clinical Practice Journal of the American Gastroenterological Association 2004; 2: 309-313

413 Schaberg T, Hauer B, Haas WH et al. Latent tuberculosis infection: recommendations for preventive therapy in adults in Germany. Pneumologie 2004; 58: 255-270

414 Balcells ME, Thomas SL, Godfrey-Faussett $P$ et al. Isoniazid preventive therapy and risk for resistant tuberculosis. Emerging Infectious Diseases 2006: 12: 744-751

415 Theis VS, Rhodes JM. Review article: minimizing tuberculosis during anti-tumour necrosis factor-alpha treatment of inflammatory bowel disease. Alimentary Pharmacology \& Therapeutics 2008; 27: 19-30

416 Schaberg T, Hauer B. Therapie der Tuberkulose und der latenten tuberkulösen Infektion - Standard für die pulmonalen und die meisten extrapulmonalen Formen. Der Klinikarzt 2008; 37: 183-188

417 Claessen MM, Lutgens MW, van Buuren HR et al. More right-sided IBDassociated colorectal cancer in patients with primary sclerosing cholangitis. Inflamm Bowel Dis 2009; 15: 1331-1336

418 Ananthakrishnan AN, McGinley EL, Binion DG. Excess hospitalisation burden associated with Clostridium difficile in patients with inflammatory bowel disease. Gut 2008; 57: 205-210

419 Dallal RM, Harbrecht BG, Boujoukas AJ et al. Fulminant Clostridium difficile: an underappreciated and increasing cause of death and complications. Ann Surg 2002; 235: 363-372

420 Meyer AM, Ramzan NN, Loftus EV et al. The diagnostic yield of stool pathogen studies during relapses of inflammatory bowel disease. Journal of Clinical Gastroenterology 2004; 38: 772-775

421 Asha NJ, Tompkins D, Wilcox MH. Comparative analysis of prevalence, risk factors, and molecular epidemiology of antibiotic-associated diarrhea due to Clostridium difficile, Clostridium perfringens, and Staphylococcus aureus. Journal of Clinical Microbiology 2006; 44: 27852791

422 Ben-Horin S, Margalit M, Bossuyt P et al. Combination immunomodulator and antibiotic treatment in patients with inflammatory bowel disease and clostridium difficile infection. Clinical Gastroenterology and Hepatology: The Official Clinical Practice Journal of the American Gastroenterological Association 2009; 7: 981-987

423 Issa M, Ananthakrishnan AN, Binion DG. Clostridium difficile and inflammatory bowel disease. Inflammatory Bowel Diseases 2008; 14 : $1432-1442$

424 Vonberg RP, Kuijper EJ, Wilcox MH et al. Infection control measures to limit the spread of Clostridium difficile. Clinical Microbiology and Infection: The Official Publication of the European Society of Clinical Microbiology and Infectious Diseases 2008; 14 (Suppl 5'): 2-20

425 Nelson $R$. Antibiotic treatment for Clostridium difficile-associated diarrhea in adults. Cochrane Database of Systematic Reviews (Online) 2007: CD004610
426 Bauer MP, Kuijper EJ, van Dissel JT. European Society of Clinical Microbiology and Infectious Diseases (ESCMID): treatment guidance document for Clostridium difficile infection (CDI). Clinical Microbiology and Infection: The Official Publication of the European Society of Clinical Microbiology and Infectious Diseases 2009; 15: 1067-1079

427 Fekety R, Silva J, Kauffman C et al. Treatment of antibiotic-associated Clostridium difficile colitis with oral vancomycin: comparison of two dosage regimens. The American Journal of Medicine 1989; 86: $15-19$

428 Gerding DN, Muto CA, Owens RC. Treatment of Clostridium difficile infection. Clinical Infectious Diseases: An Official Publication of the Infectious Diseases Society of America 2008; 46 Suppl 1: S32-42

429 Kuijper EJ, Wilcox MH. Decreased effectiveness of metronidazole for the treatment of Clostridium difficile infection? Clinical Infectious Diseases: An Official Publication of the Infectious Diseases Society of America 2008; 47: 63-65

430 Pillai A, Nelson R. Probiotics for treatment of Clostridium difficile-associated colitis in adults. Cochrane Database of Systematic Reviews (Online) 2008: CD004611

431 Musa S, Thomson S, Cowan M et al. Clostridium difficile infection and inflammatory bowel disease. Scandinavian Journal of Gastroenterology 2010; 45: 261-272

432 US Department of Agriculture. US Department of Agriculture: Food Safety for Transplant Recipients. A Need-to-Know Guide for Bone Marrow and Solid Organ Transplant Recipients. Washington, USDA 2001

433 Melmed GY, Ippoliti AF, Papadakis KA et al. Patients with inflammatory bowel disease are at risk for vaccine-preventable illnesses. The American Journal of Gastroenterology 2006; 101: 1834-1840

434 Loras C, Saro C, Gonzalez-Huix F et al. Prevalence and factors related to hepatitis B and C in inflammatory bowel disease patients in Spain: a nationwide, multicenter study. The American Journal of Gastroenterology 2009; 104: 57-63

435 Melmed GY. Vaccination strategies for patients with inflammatory bowel disease on immunomodulators and biologics. Inflammatory Bowel Diseases 2009; 15: 1410-1416

436 Wasan SK, Baker SE, Skolnik PR et al. A Practical Guide to Vaccinating the Inflammatory Bowel Disease Patient. The American Journal of Gastroenterology 2010; 105: 1231 - 1238

437 Mitteilung der Ständigen Impfkommission am RKI. Sonderdruck Nov. 2005 STIKO zu Impfungen für Patienten mit Immundefizien

438 Watson JC, Hadler SC, Dykewicz CA et al. Measles, mumps, and rubella - vaccine use and strategies for elimination of measles, rubella, and congenital rubella syndrome and control of mumps: recommendations of the Advisory Committee on Immunization Practices (ACIP) MMWR Recommendations and Reports: Morbidity and Mortality Weekly Report Recommendations and Reports/Centers for Disease Control 1998; 47: 1-57

439 Marin M, Güris D, Chaves SS et al. Prevention of varicella: recommendations of the Advisory Committee on Immunization Practices (ACIP). MMWR Recommendations and Reports: Morbidity and Mortality Weekly Report Recommendations and Reports/Centers for Disease Control 2007; 56: 1-40

440 Mitteilung der Ständigen Impfkommission (STIKO) am Robert-KochInstitut: Impfung gegen die Neue Influenza A (H1N1). Epidemiologisches Bulletin 2009; 50: 513-519

441 Nichol KL. Efficacy and effectiveness of influenza vaccination. Vaccine 2008; 26 (Suppl 4): D17-D22

442 Mamula P, Markowitz JE, Piccoli DA et al. Immune response to influenza vaccine in pediatric patients with inflammatory bowel disease. Clinical Gastroenterology and Hepatology: The Official Clinical Practice Journal of the American Gastroenterological Association 2007; 5: 851-856

443 Lu Y, Jacobson DL, Ashworth LA et al. Immune response to influenza vaccine in children with inflammatory bowel disease. The American Journal of Gastroenterology 2009; 104: 444-453

444 Institut RK. Empfehlungen der Ständigen Impfkommission (STIKO) am Robert Koch-Institut/Stand: Juli 2009

445 Vida Pérez L, Gómez Camacho F, García Sánchez V et al. Adequate rate of response to hepatitis $B$ virus vaccination in patients with inflammatory bowel disease. Medicina Clínica 2009; 132: 331-335

446 Cardell K, Akerlind B, Sällberg $M$ et al. Excellent response rate to a double dose of the combined hepatitis $A$ and $B$ vaccine in previous nonresponders to hepatitis B vaccine. The Journal of Infectious Diseases 2008; 198: 299-304 
447 Ständige Impfkomission (STIKO) am RKI, 3. Dezember 2004/Nr. 49, Begründung der STIKO für eine allgemeine Varizellenimpfung. Epidemiologisches Bulletin 2004; 49: 421 - 423

448 Kinder A, Stephens S, Mortimer N et al. Severe herpes zoster after infliximab infusion. Postgraduate Medical Journal 2004; 80: 26

449 Korelitz BI, Fuller SR, Warman JI et al. Shingles during the course of treatment with 6-mercaptopurine for inflammatory bowel disease. The American Journal of Gastroenterology 1999; 94: 424-426

450 Nobile S, Catassi C, Felici L. Herpes zoster infection followed by Henoch-Schönlein purpura in a girl receiving infliximab for ulcerative colitis. Journal of Clinical Rheumatology: Practical Reports on Rheumatic \& Musculoskeletal Diseases 2009; 15: 101

451 Impfung gegen HPV - Aktuelle Bewertung der STIKO. Epidemiologisches Bulletin 2009; 32: 319-328

452 Kane S, Khatibi B, Reddy D. Higher incidence of abnormal Pap smears in women with inflammatory bowel disease. The American Journal of Gastroenterology 2008; 103: 631-636

453 Kane S. Abnormal Pap smears in inflammatory bowel disease. Inflammatory Bowel Diseases 2008; 14: 1158-1160

454 Elkayam 0, Caspi D, Reitblatt T et al. The effect of tumor necrosis factor blockade on the response to pneumococcal vaccination in patients with rheumatoid arthritis and ankylosing spondylitis. Seminars in Arthritis and Rheumatism 2004; 33: 283-288

455 Kaine JL, Kivitz AJ, Birbara C et al. Immune responses following administration of influenza and pneumococcal vaccines to patients with rheumatoid arthritis receiving adalimumab. The Journal of Rheumatology 2007; 34: 272-279

456 Mease PJ, Ritchlin CT, Martin RW et al. Pneumococcal vaccine response in psoriatic arthritis patients during treatment with etanercept. The Journal of Rheumatology 2004; 31: 1356-1361

457 Melmed GY, Agarwal N, Frenck RW et al. Immunosuppression impairs response to pneumococcal polysaccharide vaccination in patients with inflammatory bowel disease. The American Journal of Gastroenterology 2010; 105: 148-154

458 Kapetanovic MC, Saxne T, Sjöholm A et al. Influence of methotrexate, TNF blockers and prednisolone on antibody responses to pneumococcal polysaccharide vaccine in patients with rheumatoid arthritis. Rheumatology 2006; 45: 106-111

459 Cohen $R D$, Bowie WR, Enns $R$ et al. Pulmonary actinomycosis complicating infliximab therapy for Crohn's disease. Thorax 2007; 62: 1013-1014

460 Poppers DM, Scherl EJ. Prophylaxis against Pneumocystis pneumonia in patients with inflammatory bowel disease: toward a standard of care. Inflammatory Bowel Diseases 2008; 14: 106-113

461 Green H, Paul M, Vidal L et al. Prophylaxis for Pneumocystis pneumonia (PCP) in non-HIV immunocompromised patients. Cochrane Database of Systematic Reviews (Online) 2007: CD005590

462 Rahier JF, Ben-Horin S, Chowers Y et al. European evidence-based Consensus on the prevention, diagnosis and management of opportunistic infections in inflammatory bowel disease. Journal of Crohn's and Colitis 2009; 3: 47-91

463 Ioannidis JP, Cappelleri JC, Skolnik PR et al. A meta-analysis of the relative efficacy and toxicity of Pneumocystis carinii prophylactic regimens. Archives of Internal Medicine 1996; 156: 177-188

464 Bengtson MB, Solberg IC, Aamodt G et al. Relationships between inflammatory bowel disease and perinatal factors: Both maternal and paternal disease are related to preterm birth of offspring. Inflammatory Bowel Diseases 2010; 16: 847-855

465 Blanshard C, Benhamou Y, Dohin E et al. Treatment of AIDS-associated gastrointestinal cytomegalovirus infection with foscarnet and ganciclovir: a randomized comparison. The Journal of Infectious Diseases 1995; 172: 622-628

466 Rowshani AT, Bemelman FJ, van Leeuwen EMM et al. Clinical and immunologic aspects of cytomegalovirus infection in solid organ transplant recipients. Transplantation 2005; 79: 381-386

467 de la Hoz RE, Stephens G, Sherlock C. Diagnosis and treatment approaches of CMV infections in adult patients. Journal of Clinical Virology: The Official Publication of the Pan American Society for Clinical Virology 2002; 25 (Suppl 2): S1-S12

468 Torres-Madriz G, Boucher HW. Immunocompromised hosts: perspectives in the treatment and prophylaxis of cytomegalovirus disease in solid-organ transplant recipients. Clinical Infectious Diseases: An Official Publication of the Infectious Diseases Society of America 2008; 47: 702-711
469 Preiksaitis JK, Brennan DC, Fishman J et al. Canadian society of transplantation consensus workshop on cytomegalovirus management in solid organ transplantation final report. American Journal of Transplantation: Official Journal of the American Society of Transplantation and the American Society of Transplant Surgeons 2005; 5: 218227

470 Martin DF, Sierra-Madero J, Walmsley S et al. A Controlled Trial of Valganciclovir as Induction Therapy for Cytomegalovirus Retinitis. N Engl J Med 2002; 346: 1119-1126

471 Humar A, Siegal D, Moussa G et al. A prospective assessment of valganciclovir for the treatment of cytomegalovirus infection and disease in transplant recipients. The Journal of Infectious Diseases 2005; 192: 1154-1157

472 Asberg A, Humar A, Rollag $H$ et al. Oral valganciclovir is noninferior to intravenous ganciclovir for the treatment of cytomegalovirus disease in solid organ transplant recipients. American Journal of Transplantation: Official Journal of the American Society of Transplantation and the American Society of Transplant Surgeons 2007; 7: 2106-2113

473 Len 0, Gavalda J, Aguado JM et al. Valganciclovir as treatment for cytomegalovirus disease in solid organ transplant recipients. Clinical Infectious Diseases: An Official Publication of the Infectious Diseases Society of America 2008; 46: 20-27

474 Kandiel A, Lashner B. Cytomegalovirus colitis complicating inflammatory bowel disease. The American Journal of Gastroenterology 2006; 101: 2857-2865

475 Perrottet N, Decosterd LA, Meylan P et al. Valganciclovir in adult solid organ transplant recipients: pharmacokinetic and pharmacodynamic characteristics and clinical interpretation of plasma concentration measurements. Clinical Pharmacokinetics 2009; 48: 399-418

476 Falagas ME, Snydman DR, Griffith J et al. Clinical and epidemiological predictors of recurrent cytomegalovirus disease in orthotopic liver transplant recipients. Boston Center for Liver Transplantation CMVIG Study Group. Clinical Infectious Diseases: An Official Publication of the Infectious Diseases Society of America 1997; 25: 314-317

477 Turgeon N, Fishman JA, Doran M et al. Prevention of recurrent cytomegalovirus disease in renal and liver transplant recipients: effect of oral ganciclovir. Transplant Infectious Disease: An Official Journal of the Transplantation Society 2000; $2: 2-10$

478 Lichtenstein GR, Cohen R, Yamashita B et al. Quality of life after proctocolectomy with ileoanal anastomosis for patients with ulcerative colitis. J Clin Gastroenterol 2006; 40: 669-677

479 Heuschen UA, Heuschen G, Rudek B et al. Long-term quality of life after continence-preserving proctocolectomy for ulcerative colitis and familial adenomatous polyposis. Der Chirurg; Zeitschrift fur alle Gebiete der operativen Medizen 1998; 69: 1052-1058

480 Pal S, Sahni P, Pande GK et al. Outcome following emergency surgery for refractory severe ulcerative colitis in a tertiary care centre in India. BMC gastroenterology 2005; 5: 39

481 Fowkes L, Krishna K, Menon A et al. Laparoscopic emergency and elective surgery for ulcerative colitis. Colorectal Dis 2008; 10: 373 378

482 Fazio VW, Ziv Y, Church JM et al. Ileal pouch-anal anastomoses complications and function in 1005 patients. Ann Surg 1995; 222: 120-127

483 Actis GC, Fadda M, David E et al. Colectomy rate in steroid-refractory colitis initially responsive to cyclosporin: a long-term retrospective cohort study. BMC gastroenterology 2007; 7: 13

484 Turner D, Hyams J, Markowitz J et al. Appraisal of the pediatric ulcerative colitis activity index (PUCAI). Inflamm Bowel Dis 2009; 15: 1218-1223

485 Gumaste V, Sachar DB, Greenstein AJ. Benign and malignant colorectal strictures in ulcerative colitis. Gut 1992; 33: 938-941

486 Ota H, Yamazaki K, Endoh W et al. Adenocarcinoma arising below an ileoanal anastomosis after restorative proctocolectomy for ulcerative colitis: report of a case. Surgery today $2007 ; 37$ : 596-599

487 Reiser JR, Waye JD, Janowitz HD et al. Adenocarcinoma in strictures of ulcerative colitis without antecedent dysplasia by colonoscopy. Am J Gastroenterol 1994; 89: 119-122

488 Heuschkel R, Salvestrini C, Beattie RM et al. Guidelines for the management of growth failure in childhood inflammatory bowel disease. Inflamm Bowel Dis 2008; 14: 839-849

489 Shen $B$. Impact of preoperative infliximab use on postoperative infectious complications in ulcerative colitis: the price we have to pay? Inflamm Bowel Dis 2008; 14: 1019-1021 
490 Stewart D, Chao A, Kodner I et al. Subtotal colectomy for toxic and fulminant colitis in the era of immunosuppressive therapy. Colorectal Dis 2009; 11: 184-190

491 Hait EJ, Bousvaros A, Schuman M et al. Pouch outcomes among children with ulcerative colitis treated with calcineurin inhibitors before ileal pouch anal anastomosis surgery. J Pediatr Surg 2007; 42: 31-34; discussion 34-35

492 Sorensen J, Kondrup J, Prokopowicz J et al. EuroOOPS: an international, multicentre study to implement nutritional risk screening and evaluate clinical outcome. Clin Nutr 2008; 27: 340-349

493 Weimann A, Braga M, Harsanyi L et al. ESPEN Guidelines on Enteral Nutrition: Surgery including organ transplantation. Clin Nutr 2006; 25: 224-244

494 Heuschen UA, Hinz U, Allemeyer EH et al. Risk factors for ileoanal J pouch-related septic complications in ulcerative colitis and familial adenomatous polyposis. Ann Surg 2002; 235: 207-216

495 Khuri SF, Daley J, Henderson W et al. Risk adjustment of the postoperative mortality rate for the comparative assessment of the quality of surgical care: results of the National Veterans Affairs Surgical Risk Study. Journal of the American College of Surgeons 1997; 185: 315 327

496 Malone DL, Genuit T, Tracy JK et al. Surgical site infections: reanalysis of risk factors. J Surg Res 2002; 103: 89-95

497 Fazio VW, Tjandra JJ. Transanal mucosectomy. Ileal pouch advancement for anorectal dysplasia or inflammation after restorative proctocolectomy. Dis Colon Rectum 1994; 37: 1008-1011

498 Lavery IC, Sirimarco MT, Ziv Y et al. Anal canal inflammation after ileal pouch-anal anastomosis. The need for treatment. Dis Colon Rectum 1995; 38: 803-806

499 O'Riordain MG, Fazio VW, Lavery IC et al. Incidence and natural history of dysplasia of the anal transitional zone after ileal pouch-anal anastomosis: results of a five-year to ten-year follow-up. Dis Colon Rectum 2000; 43: 1660-1665

500 van Bodegraven AA, Boer RO, Lourens J et al. Distribution of mesalazine enemas in active and quiescent ulcerative colitis. Aliment Pharmacol Ther 1996; 10: 327-332

501 Litzendorf ME, Stucchi AF, Wishnia S et al. Completion mucosectomy for retained rectal mucosa following restorative proctocolectomy with double-stapled ileal pouch-anal anastomosis. J Gastrointest Surg 2010; 14: 562-569

502 Ault GT, Nunoo-Mensah JW, Johnson L et al. Adenocarcinoma arising in the middle of ileoanal pouches: report of five cases. Dis Colon Rectum 2009; 52: 538-541

503 Linehan G, Cahill RA, Kalimuthu SN et al. Adenocarcinoma arising in the ileoanal pouch after restorative proctocolectomy for familial adenomatous polyposis. Int J Colorectal Dis 2008; 23: 329-330

504 Naik VS, Patil SB, Scholefield J et al. Adenocarcinoma arising in a background of chronic atrophic pouchitis in an ileoanal pouch for ulcerative colitis. Histopathology 2008; 53: 354-358

505 Kaplan GG, McCarthy EP, Ayanian JZ et al. Impact of hospital volume on postoperative morbidity and mortality following a colectomy for ulcerative colitis. Gastroenterology 2008; 134: 680-687

506 Nessar G, Fazio VW, Tekkis P et al. Long-term outcome and quality of life after continent ileostomy. Dis Colon Rectum 2006; 49: 336-344

507 Berndtsson I, Lindholm E, Ekman I. Thirty years of experience living with a continent ileostomy: bad restrooms - not my reservoir - decide my life. J Wound Ostomy Continence Nurs 2005; 32: 321-326; quiz $327-328$

508 Litle VR, Barbour S, Schrock TR et al. The continent ileostomy: longterm durability and patient satisfaction. J Gastrointest Surg 1999; 3: 625-632

509 Castillo E, Thomassie LM, Whitlow CB et al. Continent ileostomy: current experience. Dis Colon Rectum 2005; 48: 1263-1268

510 Leijonmarck CE, Lofberg $R$, Ost A et al. Long-term results of ileorectal anastomosis in ulcerative colitis in Stockholm County. Dis Colon Rectum 1990; 33: 195-200

511 Elton C, Makin G, Hitos $\mathrm{K}$ et al. Mortality, morbidity and functional outcome after ileorectal anastomosis. Br J Surg 2003; 90: 59-65

512 Maartense S, Dunker MS, Slors JF et al. Hand-assisted laparoscopic versus open restorative proctocolectomy with ileal pouch anal anastomosis: a randomized trial. Ann Surg 2004; 240: 984-991; discussion $991-982$

513 Tilney HS, Lovegrove RE, Heriot AG et al. Comparison of short-term outcomes of laparoscopic vs open approaches to ileal pouch surgery. Int J Colorectal Dis 2007; 22: 531-542
514 Chung TP, Fleshman JW, Birnbaum EH et al. Laparoscopic vs. open total abdominal colectomy for severe colitis: impact on recovery and subsequent completion restorative proctectomy. Dis Colon Rectum 2009; 52: 4-10

515 Geboes K, Colombel JF, Greenstein A et al. Indeterminate colitis: a review of the concept - what's in a name? Inflamm Bowel Dis 2008; 14: 850-857

516 Pishori T, Dinnewitzer A, Zmora 0 et al. Outcome of patients with indeterminate colitis undergoing a double-stapled ileal pouch-anal anastomosis. Dis Colon Rectum 2004; 47: 717-721

517 Delaney CP, Remzi FH, Gramlich T et al. Equivalent function, quality of life and pouch survival rates after ileal pouch-anal anastomosis for indeterminate and ulcerative colitis. Ann Surg 2002; 236: 43-48

518 Regimbeau JM, Panis Y, Pocard $M$ et al. Long-term results of ileal pouch-anal anastomosis for colorectal Crohn's disease. Dis Colon Rectum 2001; 44: 769-778

519 Malaty HM, Fan X, Opekun AR et al. Rising incidence of inflammatory bowel disease among children: a 12-year study. J Pediatr Gastroenterol Nutr 2010; 50: 27-31

520 Sandborn WJ, Tremaine WJ, Batts KP et al. Pouchitis after ileal pouchanal anastomosis: a Pouchitis Disease Activity Index. Mayo Clin Proc 1994; 69: 409-415

521 Tiainen J, Matikainen M. Long-term clinical outcome and anemia after restorative proctocolectomy for ulcerative colitis. Scand J Gastroenterol 2000; 35: 1170-1173

522 Meagher AP, Farouk R, Dozois RR et al. J ileal pouch-anal anastomosis for chronic ulcerative colitis: complications and long-term outcome in 1310 patients. Br J Surg 1998; 85: 800-803

523 Hurst RD, Chung TP, Rubin M et al. The implications of acute pouchitis on the long-term functional results after restorative proctocolectomy. Inflamm Bowel Dis 1998; 4: 280-284

524 Tiainen J, Matikainen M, Aitola P et al. Histological and macroscopic changes in the pelvic pouch: long-term follow up after restorative proctocolectomy for ulcerative colitis (UC). Colorectal Dis 2001; 3: 28-32

525 Tiainen J, Matikainen M. Health-related quality of life after ileal Jpouch-anal anastomosis for ulcerative colitis: long-term results. Scand J Gastroenterol 1999; 34: 601-605

526 Seidel SA, Peach SE, Newman M et al. Ileoanal pouch procedures: clinical outcomes and quality-of-life assessment. The American surgeon 1999; 65: 40-46

527 Heuschen UA, Autschbach F, Allemeyer EH et al. Long-term follow-up after ileoanal pouch procedure: algorithm for diagnosis, classification, and management of pouchitis. Dis Colon Rectum 2001; 44 487-499

528 Stahlberg D, Gullberg K, Liljeqvist $L$ et al. Pouchitis following pelvic pouch operation for ulcerative colitis. Incidence, cumulative risk, and risk factors. Dis Colon Rectum 1996; 39: 1012-1018

529 Lohmuller JL, Pemberton JH, Dozois RR et al. Pouchitis and extraintestinal manifestations of inflammatory bowel disease after ileal pouchanal anastomosis. Ann Surg 1990; 211: 622-627; discussion $627-$ 629

530 Nicholls RJ, Banerjee AK. Pouchitis: risk factors, etiology, and treatment. World J Surg 1998; 22: 347-351

531 Penna $C$, Dozois $R$, Tremaine $W$ et al. Pouchitis after ileal pouch-anal anastomosis for ulcerative colitis occurs with increased frequency in patients with associated primary sclerosing cholangitis. Gut 1996 38: 234-239

532 Shen B, Achkar JP, Lashner BA et al. Irritable pouch syndrome: a new category of diagnosis for symptomatic patients with ileal pouch-anal anastomosis. Am J Gastroenterol 2002; 97: 972-977

533 Thoeni RF, Fell SC, Engelstad B et al. Ileoanal pouches: comparison of $\mathrm{CT}$, scintigraphy, and contrast enemas for diagnosing postsurgical complications. Ajr 1990; 154: 73-78

534 Libicher M, Scharf J, Wunsch A et al. MRI of pouch-related fistulas in ulcerative colitis after restorative proctocolectomy. Journal of computer assisted tomography 1998; 22: 664-668

535 Hrung JM, Levine MS, Rombeau JL et al. Total proctocolectomy and ileoanal pouch: the role of contrast studies for evaluating postoperative leaks. Abdom Imaging 1998; 23: 375-379

536 Solomon MJ, McLeod RS, O'Connor BI et al. Assessment of peripouch inflammation after ileoanal anastomosis using endoluminal ultrasonography. Dis Colon Rectum 1995; 38: 182-187 
537 Sandborn W, McLeod R, Jewell D. Pharmacotherapy for inducing and maintaining remission in pouchitis. Cochrane Database Syst Rev 2000: CD001176

538 Sandborn WJ, McLeod R, Jewell DP. Medical therapy for induction and maintenance of remission in pouchitis: a systematic review. Inflamm Bowel Dis 1999; 5: 33-39

539 Gionchetti P, Rizzello F, Venturi A et al. Oral bacteriotherapy as maintenance treatment in patients with chronic pouchitis: a double-blind, placebo-controlled trial. Gastroenterology 2000; 119: 305-309

540 Hurst RD, Molinari M, Chung TP et al. Prospective study of the incidence, timing and treatment of pouchitis in 104 consecutive patients after restorative proctocolectomy. Arch Surg 1996; 131: 497-500; discussion $501-492$

541 Svaninger G, Nordgren S, Oresland T et al. Incidence and characteristics of pouchitis in the Kock continent ileostomy and the pelvic pouch. Scand J Gastroenterol 1993; 28: 695-700

542 Rauh SM, SchoetzJr DJ, Roberts PL et al. Pouchitis - is it a wastebasket diagnosis? Dis Colon Rectum 1991; 34: 685-689

543 Shen B, Achkar JP, Lashner BA et al. A randomized clinical trial of ciprofloxacin and metronidazole to treat acute pouchitis. Inflamm Bowel Dis 2001; 7: 301-305

544 Nygaard K, Bergan T, Bjorneklett A et al. Topical metronidazole treatment in pouchitis. Scand J Gastroenterol 1994; 29: 462-467

545 Mimura T, Rizzello F, Helwig U et al. Four-week open-label trial of metronidazole and ciprofloxacin for the treatment of recurrent or refractory pouchitis. Aliment Pharmacol Ther 2002; 16: 909-917

546 Gionchetti P, Rizzello F, Venturi A et al. Antibiotic combination therapy in patients with chronic, treatment-resistant pouchitis. Aliment Pharmacol Ther 1999; 13: 713-718

547 Gionchetti P, Rizzello F, Helwig $U$ et al. Prophylaxis of pouchitis onset with probiotic therapy: a double-blind, placebo-controlled trial. Gastroenterology 2003; 124: 1202-1209

548 Holubar SD, Cima RR, Sandborn WJ et al. Treatment and prevention of pouchitis after ileal pouch-anal anastomosis for chronic ulcerative colitis. Cochrane Database Syst Rev 6: CD001176

549 Gullberg $K$, Lindforss $U$, Zetterquist $H$ et al. Cancer risk assessment in long-standing pouchitis. DNA aberrations are rare in transformed neoplastic pelvic pouch mucosa. Int J Colorectal Dis 2002; 17: 92-97

550 Borjesson L, Willen $R$, Haboubi $N$ et al. The risk of dysplasia and cancer in the ileal pouch mucosa after restorative proctocolectomy for ulcerative proctocolitis is low: a long-term term follow-up study. Colorectal Dis 2004; 6: 494-498

551 Eaden JA, Abrams KR, Mayberry JF. The risk of colorectal cancer in ulcerative colitis: a meta-analysis. Gut 2001; 48: 526-535

552 Lakatos L, Mester G, Erdelyi Z et al. Risk factors for ulcerative colitisassociated colorectal cancer in a Hungarian cohort of patients with ulcerative colitis: results of a population-based study. Inflamm Bowel Dis 2006; 12: 205-211

553 Soderlund S, Brandt L, Lapidus A et al. Decreasing time-trends of colorectal cancer in a large cohort of patients with inflammatory bowel disease. Gastroenterology 2009; 136: 1561-1567; quiz 1818- 1569

554 Winther KV, Jess T, Langholz E et al. Long-term risk of cancer in ulcerative colitis: a population-based cohort study from Copenhagen County. Clin Gastroenterol Hepatol 2004; 2: 1088-1095

555 Gupta RB, Harpaz N, Itzkowitz S et al. Histologic inflammation is a risk factor for progression to colorectal neoplasia in ulcerative colitis: a cohort study. Gastroenterology 2007; 133: 1099-1105; quiz 1340 1341

556 Velayos FS, LoftusJr EV, Jess T et al. Predictive and protective factors associated with colorectal cancer in ulcerative colitis: A case-control study. Gastroenterology 2006; 130: 1941-1949

557 Rutter MD, Saunders BP, Wilkinson KH et al. Thirty-year analysis of a colonoscopic surveillance program for neoplasia in ulcerative colitis. Gastroenterology 2006; 130: 1030-1038

558 Heuschen UA, Hinz U, Allemeyer EH et al. Backwash ileitis is strongly associated with colorectal carcinoma in ulcerative colitis. Gastroenterology $2001 ; 120: 841-847$

559 Collins PD, Mpofu C, Watson AJ et al. Strategies for detecting colon cancer and/or dysplasia in patients with inflammatory bowel disease. Cochrane Database of Systematic Reviews (Online) 2006: CD000279

560 Lutgens MW, Vleggaar FP, Schipper ME et al. High frequency of early colorectal cancer in inflammatory bowel disease. Gut 2008; 57 : 1246-1251
561 Connell WR, Lennard-Jones JE, Williams CB et al. Factors affecting the outcome of endoscopic surveillance for cancer in ulcerative colitis. Gastroenterology 1994; 107: 934-944

562 Scarpa M, van Koperen PJ, Ubbink DT et al. Systematic review of dysplasia after restorative proctocolectomy for ulcerative colitis. $\mathrm{Br}$ J Surg 2007; 94: 534-545

563 Thomas-Gibson S, Rogers P, Cooper S et al. Judgement of the quality of bowel preparation at screening flexible sigmoidoscopy is associated with variability in adenoma detection rates. Endoscopy 2006; 38: 456-460

564 Toruner M, Harewood GC, LoftusJr EV et al. Endoscopic factors in the diagnosis of colorectal dysplasia in chronic inflammatory bowel disease. Inflamm Bowel Dis 2005; 11: 428-434

565 Rubin DT, Rothe JA, Hetzel JT et al. Are dysplasia and colorectal cancer endoscopically visible in patients with ulcerative colitis? Gastrointest Endosc 2007; 65: 998-1004

566 Rutter MD, Saunders BP, Schofield G et al. Pancolonic indigo carmine dye spraying for the detection of dysplasia in ulcerative colitis. Gut 2004; 53: 256-260

567 Rubin CE, Haggitt RC, Burmer GC et al. DNA aneuploidy in colonic biopsies predicts future development of dysplasia in ulcerative colitis. Gastroenterology 1992; 103: 1611-1620

568 Kaltz B, Bokemeyer B, Hoffmann J et al. Surveillance colonoscopy in ulcerative colitis patients in Germany. Z Gastroenterol 2007; 45: 325331

569 Marion JF, Waye JD, Present DH et al. Chromoendoscopy-targeted biopsies are superior to standard colonoscopic surveillance for detecting dysplasia in inflammatory bowel disease patients: a prospective endoscopic trial. Am J Gastroenterol 2008; 103: 2342-2349

570 Dekker E, van den Broek FJ, Reitsma JB et al. Narrow-band imaging compared with conventional colonoscopy for the detection of dysplasia in patients with longstanding ulcerative colitis. Endoscopy 2007; 39: $216-221$

571 Odze RD, Goldblum J, Noffsinger A et al. Interobserver variability in the diagnosis of ulcerative colitis-associated dysplasia by telepathology. Mod Pathol 2002; 15: 379-386

572 Odze RD, Farraye FA, Hecht JL et al. Long-term follow-up after polypectomy treatment for adenoma-like dysplastic lesions in ulcerative colitis. Clin Gastroenterol Hepatol 2004; 2: 534-541

573 Velayos FS, Terdiman JP, Walsh JM. Effect of 5-aminosalicylate use on colorectal cancer and dysplasia risk: a systematic review and metaanalysis of observational studies. The American Journal of Gastroenterology 2005; 100: 1345-1353

574 Pardi DS, LoftusJr EV, Kremers WK et al. Ursodeoxycholic acid as a chemopreventive agent in patients with ulcerative colitis and primary sclerosing cholangitis. Gastroenterology 2003; 124: 889-893

575 Tung BY, Emond MJ, Haggitt RC et al. Ursodiol use is associated with lower prevalence of colonic neoplasia in patients with ulcerative colitis and primary sclerosing cholangitis. Ann Intern Med 2001; 134 : 89-95

576 Lindor KD, Kowdley KV, Luketic VA et al. High-dose ursodeoxycholic acid for the treatment of primary sclerosing cholangitis. Hepatology 2009; 50: 808-814

577 Matula S, Croog V, Itzkowitz S et al. Chemoprevention of colorectal neoplasia in ulcerative colitis: the effect of 6 -mercaptopurine. Clinical Gastroenterology and Hepatology: The Official Clinical Practice Journal of the American Gastroenterological Association 2005; 3: 10151021

578 O'Donovan P, Perrett CM, Zhang X et al. Azathioprine and UVA light generate mutagenic oxidative DNA damage. Science 2005; 309: 18711874

579 Perrett CM, Walker SL, O'Donovan Pet al. Azathioprine treatment photosensitizes human skin to ultraviolet A radiation. Br J Dermatol 2008: 159: 198-204

580 Kinlen $L J$. Incidence of cancer in rheumatoid arthritis and other disorders after immunosuppressive treatment. Am J Med 1985; 78: 44-49

581 Dougados $M$, van der Linden S, Juhlin R et al. The European Spondylarthropathy Study Group preliminary criteria for the classification of spondylarthropathy. Arthritis Rheum 1991; 34: 1218-1227

582 Orchard TR, Wordsworth BP, Jewell DP. Peripheral arthropathies in inflammatory bowel disease: their articular distribution and natural history. Gut 1998; 42: 387-391

583 Greenstein AJ, Janowitz HD, Sachar DB. The extra-intestinal complications of Crohn's disease and ulcerative colitis: a study of 700 patients. Medicine 1976; 55: 401-412 
584 Gran JT, Husby G. Joint manifestations in gastrointestinal diseases. 1. Pathophysiological aspects, ulcerative colitis and Crohn's disease. Dig Dis 1992; 10: 274-294

585 de Vlam K, Mielants H, Cuvelier C et al. Spondyloarthropathy is underestimated in inflammatory bowel disease: prevalence and HLA association. J Rheumatol 2000; 27: 2860-2865

586 Palm 0, Moum B, Jahnsen J et al. The prevalence and incidence of peripheral arthritis in patients with inflammatory bowel disease, a prospective population-based study (the IBSEN study). Rheumatology 2001; 40: 1256-1261

587 Palm 0, Moum B, Ongre A et al. Prevalence of ankylosing spondylitis and other spondyloarthropathies among patients with inflammatory bowel disease: a population study (the IBSEN study). J Rheumatol 2002; 29: 511-515

588 Protzer U, Duchmann R, Hohler T et al. Enteropathic spondylarthritis in chronic inflammatory bowel diseases: prevalence, manifestation pattern and HLA association. Med Klin 1996; 91: 330-335

589 Braun J, Bollow M, Remlinger $G$ et al. Prevalence of spondylarthropathies in HLA-B27 positive and negative blood donors. Arthritis Rheum 1998; 41: 58-67

590 Thomas PD, Keat AC, Forbes A et al. Extraintestinal manifestations of ulcerative colitis following restorative proctocolectomy. Eur J Gastroenterol Hepatol 1999; 11: 1001-1005

591 Scarpa R, del Puente A, D'Arienzo A et al. The arthritis of ulcerative colitis: clinical and genetic aspects. J Rheumatol 1992; 19: 373-377

592 Jones G, Halbert J, Crotty M et al. The effect of treatment on radiological progression in rheumatoid arthritis: a systematic review of randomized placebo-controlled trials. Rheumatology 2003; 42: 6-13

593 Dougados M, vam der Linden S, Leirisalo-Repo $M$ et al. Sulfasalazine in the treatment of spondylarthropathy. A randomized, multicenter, double-blind, placebo-controlled study. Arthritis Rheum 1995; 38: 618-627

594 Clegg DO, Reda DJ, Weisman MH et al. Comparison of sulfasalazine and placebo in the treatment of ankylosing spondylitis. A Department of Veterans Affairs Cooperative Study. Arthritis Rheum 1996; 39: 2004 2012

595 Kefalakes H, Stylianides TJ, Amanakis G et al. Exacerbation of inflammatory bowel diseases associated with the use of nonsteroidal antiinflammatory drugs: myth or reality? Eur J Clin Pharmacol 2009; 65: 963-970

596 Felder JB, Korelitz BI, Rajapakse R et al. Effects of nonsteroidal antiinflammatory drugs on inflammatory bowel disease: a case-control study. Am J Gastroenterol 2000; 95: 1949-1954

597 Bonner GF, Fakhri A, Vennamaneni SR. A long-term cohort study of nonsteroidal anti-inflammatory drug use and disease activity in outpatients with inflammatory bowel disease. Inflamm Bowel Dis 2004; 10: 751-757

598 El Miedany Y, Youssef S, Ahmed I et al. The gastrointestinal safety and effect on disease activity of etoricoxib, a selective cox-2 inhibitor in inflammatory bowel diseases. Am J Gastroenterol 2006; 101: 311317

599 Freitas J, Farricha V, Nascimento I et al. Rofecoxib: a possible cause of acute colitis. J Clin Gastroenterol 2002; 34: 451-453

600 Herfarth $H$, Obermeier $F$, Andus $T$ et al. Improvement of arthritis and arthralgia after treatment with infliximab (Remicade) in a German prospective, open-label, multicenter trial in refractory Crohn's disease. Am J Gastroenterol 2002; 97: 2688-2690

601 Braun J, Brandt J, Listing J et al. Treatment of active ankylosing spondylitis with infliximab: a randomised controlled multicentre trial. Lancet 2002; 359: 1187-1193

602 Van Den Bosch F, Kruithof E, Baeten D et al. Randomized double-blind comparison of chimeric monoclonal antibody to tumor necrosis factor alpha (infliximab) versus placebo in active spondylarthropathy. Arthritis Rheum 2002; 46: 755-765

603 van der Heijde D, Kivitz A, Schiff MH et al. Efficacy and safety of adalimumab in patients with ankylosing spondylitis: results of a multicenter, randomized, double-blind, placebo-controlled trial. Arthritis Rheum 2006; 54: 2136-2146

604 Lambert RG, Salonen D, Rahman P et al. Adalimumab significantly reduces both spinal and sacroiliac joint inflammation in patients with ankylosing spondylitis: a multicenter, randomized, double-blind, placebo-controlled study. Arthritis Rheum 2007; 56: 4005-4014

605 Zochling J, van der Heijde D, Dougados M et al. Current evidence for the management of ankylosing spondylitis: a systematic literature re- view for the ASAS/EULAR management recommendations in ankylosing spondylitis. Ann Rheum Dis 2006; 65: 423-432

606 Berstad AE, Aabakken L, Smith HJ et al. Diagnostic accuracy of magnetic resonance and endoscopic retrograde cholangiography in primary sclerosing cholangitis. Clin Gastroenterol Hepatol 2006; 4: 514-520

607 Moff SL, Kamel IR, Eustace J et al. Diagnosis of primary sclerosing cholangitis: a blinded comparative study using magnetic resonance cholangiography and endoscopic retrograde cholangiography. Gastrointest Endosc 2006; 64: 219-223

608 Abbas G, Lindor KD. Cholangiocarcinoma in primary sclerosing cholangitis. J Gastrointest Cancer 2009; 40: 19-25

609 Lee YM, Kaplan MM Practice Guideline Committee of the ACG, American College of Gastroenterology. Management of primary sclerosing cholangitis. Am J Gastroenterol 2002; 97: 528-534

610 Mitchell SA, Bansi DS, Hunt N et al. A preliminary trial of high-dose ursodeoxycholic acid in primary sclerosing cholangitis. Gastroenterology 2001; 121: 900-907

611 Baluyut AR, Sherman S, Lehman GA et al. Impact of endoscopic therapy on the survival of patients with primary sclerosing cholangitis. Gastrointest Endosc 2001; 53: 308-312

612 Gotthardt DN, Rudolph G, Kloters-Plachky P et al. Endoscopic dilation of dominant stenoses in primary sclerosing cholangitis: outcome after long-term treatment. Gastrointest Endosc 2010; 71: 527-534

613 Ruocco E, Sangiuliano S, Gravina AG et al. Pyoderma gangrenosum: an updated review. J Eur Acad Dermatol Venereol 2009; 23: 1008-1017

614 Larsen S, Bendtzen K, Nielsen $\mathrm{OH}$. Extraintestinal manifestations of in flammatory bowel disease: epidemiology, diagnosis, and management. Ann Med 2010; 42: 97-114

615 Yuksel I, Basar O, Ataseven H et al. Mucocutaneous manifestations in inflammatory bowel disease. Inflamm Bowel Dis 2009; 15: 546-550

616 Yilmaz S, Aydemir E, Maden A et al. The prevalence of ocular involvement in patients with inflammatory bowel disease. Int J Colorectal Dis 2007; 22: 1027-1030

617 Soukiasian SH, Foster CS, Raizman MB. Treatment strategies for scleritis and uveitis associated with inflammatory bowel disease. Am J Ophthalmol 1994; 118: 601-611

618 Mintz R, Feller ER, Bahr RL et al. Ocular manifestations of inflammatory bowel disease. Inflamm Bowel Dis 2004; 10: 135-139

619 de Vries J, Baarsma GS, Zaal MJ et al. Cyclosporin in the treatment of severe chronic idiopathic uveitis. Br J Ophthalmol 1990; 74: 344-349

620 Joseph A, Raj D, Dua HS et al. Infliximab in the treatment of refractory posterior uveitis. Ophthalmology 2003; 110: 1449-1453

621 Reinshagen $M$. Osteoporosis in inflammatory bowel disease. J Crohns Colitis 2008; 2: 202-207

622 Lange $U$, Muller-Ladner U. Glucocorticoid induced osteoporosis. Z Rheumatol 2007; 66: 129-136; quiz 137 - 128

623 Drossman DA. Presidential address: Gastrointestinal illness and the biopsychosocial model. Psychosom Med 1998; 60: 258-267

624 Engel GL. The need for a new medical model: a challenge for biomedicine. Science 1977; 196: 129-136

625 Graff LA, Walker JR, Bernstein CN. Depression and anxiety in inflammatory bowel disease: a review of comorbidity and management. Inflamm Bowel Dis 2009; 15: 1105-1118

626 Camara RJ, Ziegler R, Begre S et al. The role of psychological stress in inflammatory bowel disease: quality assessment of methods of 18 prospective studies and suggestions for future research. Digestion 2009; 80: 129-139

627 Goodhand JR, Wahed M, Rampton DS. Management of stress in inflammatory bowel disease: a therapeutic option? Expert Rev Gastroenterol Hepatol 2009; 3: 661-679

628 Nigro G, Angelini G, Grosso SB et al. Psychiatric predictors of noncompliance in inflammatory bowel disease: psychiatry and compliance. J Clin Gastroenterol 2001; 32: 66-68

629 Gomez-Gil E, Vidal A, Panes J et al. Relationship between patient's subjective stress perception and the course of inflammatory bowel disease. Gastroenterol Hepatol 2003; 26: 411-416

630 Mikocka-Walus AA, Turnbull DA, Moulding NT et al. Controversies surrounding the comorbidity of depression and anxiety in inflammatory bowel disease patients: a literature review. Inflamm Bowel Dis 2007; 13: $225-234$

631 Porcelli P, Leoci C, Guerra V. A prospective study of the relationship between disease activity and psychologic distress in patients with inflammatory bowel disease. Scand J Gastroenterol 1996; 31: 792-796

632 Guthrie E, Jackson J, Shaffer J et al. Psychological disorder and severity of inflammatory bowel disease predict health-related quality of life 
in ulcerative colitis and Crohn's disease. Am J Gastroenterol 2002; 97 : 1994-1999

633 Rubin GP, Hungin AP, Chinn DJ et al. Quality of life in patients with established inflammatory bowel disease: a UK general practice survey. Aliment Pharmacol Ther 2004; 19: 529-535

634 Bernklev T, Jahnsen J, Lygren I et al. Health-related quality of life in patients with inflammatory bowel disease measured with the short form-36: psychometric assessments and a comparison with general population norms. Inflamm Bowel Dis 2005; 11: 909-918

635 Janke KH, Klump B, Gregor M et al. Determinants of life satisfaction in inflammatory bowel disease. Inflamm Bowel Dis 2005; 11: 272-286

636 Han SW, McColl E, Barton JR et al. Predictors of quality of life in ulcerative colitis: the importance of symptoms and illness representations. Inflamm Bowel Dis 2005; 11: 24-34

637 Casellas F, Arenas JI, Baudet JS et al. Impairment of health-related quality of life in patients with inflammatory bowel disease: a Spanish multicenter study. Inflamm Bowel Dis 2005; 11: 488-496

638 Graff LA, Walker JR, Lix L et al. The relationship of inflammatory bowel disease type and activity to psychological functioning and quality of life. Clin Gastroenterol Hepatol 2006; 4: 1491-1501

639 de Boer AG, Sprangers MA, Bartelsman JF et al. Predictors of health care utilization in patients with inflammatory bowel disease: a longitudinal study. Eur J Gastroenterol Hepatol 1998; 10: 783-789

640 Mittermaier C, Dejaco C, Waldhoer T et al. Impact of depressive mood on relapse in patients with inflammatory bowel disease: a prospective 18-month follow-up study. Psychosom Med 2004; 66: 79-84

641 Hauser W, Janke KH, Stallmach A. Mental disorder and psychologic distress in patients with ulcerative colitis after ileal pouch-anal anastomosis. Dis Colon Rectum 2005; 48: 952-962

642 Mussell M, Bocker U, Nagel N et al. Predictors of disease-related concerns and other aspects of health-related quality of life in outpatients with inflammatory bowel disease. Eur J Gastroenterol Hepatol 2004; 16: 1273-1280

643 Simren M, Axelsson J, Gillberg R et al. Quality of life in inflammatory bowel disease in remission: the impact of IBS-like symptoms and associated psychological factors. Am J Gastroenterol 2002; 97: 389-396

644 Minderhoud IM, Oldenburg B, Wismeijer JA et al. IBS-like symptoms in patients with inflammatory bowel disease in remission; relationships with quality of life and coping behavior. Dig Dis Sci 2004; 49: 469-474

645 Ansari R, Attari F, Razjouyan H et al. Ulcerative colitis and irritable bowel syndrome: relationships with quality of life. Eur J Gastroenterol Hepatol 2008; 20: 46-50

646 Farrokhyar F, Marshall JK, Easterbrook B et al. Functional gastrointestinal disorders and mood disorders in patients with inactive inflammatory bowel disease: prevalence and impact on health. Inflamm Bowel Dis 2006; 12: 38-46

647 Spiller R. Clinical update: irritable bowel syndrome. Lancet 2007; 369: 1586-1588

648 Guyatt G, Mitchell A, Irvine EJ et al. A new measure of health status for clinical trials in inflammatory bowel disease. Gastroenterology 1989; 96: 804-810

649 Janke KH, Klump B, Steder-Neukamm U et al. Validation of the German version of the Inflammatory Bowel Disease Questionnaire (Competence Network IBD, IBDQ-D). Psychother Psychosom Med Psychol 2006; 56: 291-298

650 Irvine EJ, Zhou Q Thompson AK. The Short Inflammatory Bowel Disease Questionnaire: a quality of life instrument for community physicians managing inflammatory bowel disease. CCRPT Investigators. Canadian Crohn's Relapse Prevention Trial. Am J Gastroenterol 1996; 91: 1571-1578

651 Rose $M$, Fliege $H$, Hildebrandt $M$ et al. Validation of the new German translation version of the „Short Inflammatory Bowel Disease Questionnaire“ (SIBDQ). Z Gastroenterol 2000; 38: 277-286

652 Drossman DA, Leserman J, Li ZM et al. The rating form of IBD patient concerns: a new measure of health status. Psychosom Med 1991; 53: 701-712

653 Kunzendorf S, Jantschek G, Straubinger K et al. The Luebeck interview for psychosocial screening in patients with inflammatory bowel disease. Inflamm Bowel Dis 2007; 13: 33-41

654 Kroenke K, Spitzer RL, Williams JB. The Patient Health Questionnaire2: validity of a two-item depression screener. Med Care 2003; 41: 1284-1292

655 Herrmann-Lingen C, Buss U, Snaith RP. Hospital Anxiety and Depression Scale - deutsche Version (HADS-D). Bern: Hans Huber, 1995
656 Sewitch MJ, Abrahamowicz M, Bitton A et al. Psychological distress, social support, and disease activity in patients with inflammatory bowel disease. Am J Gastroenterol 2001; 96: 1470-1479

657 Sewitch MJ, Abrahamowicz M, Bitton A et al. Psychosocial correlates of patient-physician discordance in inflammatory bowel disease. Am J Gastroenterol 2002; 97: 2174-2183

658 Hjortswang H, Jarnerot G, Curman B et al. The influence of demographic and disease-related factors on health-related quality of life in patients with ulcerative colitis. Eur J Gastroenterol Hepatol 2003; 15: 1011-1020

659 Saibeni S, Cortinovis I, Beretta L et al. Gender and disease activity influence health-related quality of life in inflammatory bowel diseases. Hepatogastroenterology 2005; 52: 509-515

660 Timmer A, Bauer A, Kemptner D et al. Determinants of male sexual function in inflammatory bowel disease: a survey-based crosssectional analysis in 280 men. Inflamm Bowel Dis 2007; 13: 12361243

661 Fuller-Thomson E, Sulman J. Depression and inflammatory bowel disease: findings from two nationally representative Canadian surveys. Inflamm Bowel Dis 2006; 12: 697-707

662 Mussell M, Bocker U, Nagel $N$ et al. Reducing psychological distress in patients with inflammatory bowel disease by cognitive-behavioural treatment: exploratory study of effectiveness. Scand J Gastroenterol 2003; 38: 755-762

663 Mountifield R, Bampton P, Prosser R et al. Fear and fertility in inflammatory bowel disease: a mismatch of perception and reality affects family planning decisions. Inflamm Bowel Dis 2009; 15: 720-725

664 Miehsler W, Weichselberger M, Offerlbauer-Ernst A et al. Which patients with IBD need psychological interventions? A controlled study. Inflamm Bowel Dis 2008; 14: 1273-1280

665 Moser G, Jantschek G. Ulcerative colitis. Psychosomatics. Z Gastroenterol 2004; 42: 1029-1030

666 Caprilli R, Gassull MA, Escher JC et al. European evidence based consensus on the diagnosis and management of Crohn's disease: special situations. Gut 2006; 55 (Suppl 1): i36-i58

667 Moser G, Tillinger W, Sachs G et al. Disease-related worries and concerns: a study on out-patients with inflammatory bowel disease. Eur J Gastroenterol Hepatol 1995; 7: 853-858

668 Casellas F, Fontanet G, Borruel $N$ et al. The opinion of patients with inflammatory bowel disease on healthcare received. Rev Esp Enferm Dig 2004; 96: 174-184

669 Kennedy AP, Nelson E, Reeves D et al. A randomised controlled trial to assess the effectiveness and cost of a patient orientated self management approach to chronic inflammatory bowel disease. Gut 2004; 53: $1639-1645$

670 Robinson A, Thompson DG, Wilkin D et al. Guided self-management and patient-directed follow-up of ulcerative colitis: a randomised trial. Lancet 2001; 358: 976-981

671 Borgaonkar MR, Townson G, Donnelly $M$ et al. Providing disease-related information worsens health-related quality of life in inflammatory bowel disease. Inflamm Bowel Dis 2002; 8: 264-269

672 Elkjaer M, Burisch J, Avnstrom S et al. Development of a Web-based concept for patients with ulcerative colitis and 5-aminosalicylic acid treatment. Eur J Gastroenterol Hepatol 2010; 22: 695-704

673 Lange A, Haslbeck E, Andus T et al. Ambulatory education of patient with Crohn disease/ulcerative colitis. Z Gastroenterol 1996; 34: 411-415

674 von Wietersheim J, Jantschek G, Sommer W et al. Education of patients with inflammatory bowel diseases. Wien Med Wochenschr 1999; 149: $352-354$

675 Larsson K, Sundberg Hjelm M, Karlbom U et al. A group-based patient education programme for high-anxiety patients with Crohn disease or ulcerative colitis. Scand J Gastroenterol 2003; 38: 763-769

676 Jaghult S, Larson J, Wredling $R$ et al. A multiprofessional education programme for patients with inflammatory bowel disease: a randomized controlled trial. Scand J Gastroenterol 2007: 42: 1452-1459

677 Maunder RG, Esplen MJ. Supportive-expressive group psychotherapy for persons with inflammatory bowel disease. Can J Psychiatry 2001; 46: 622-626

678 von Wietersheim J, Kessler H. Psychotherapy with chronic inflammatory bowel disease patients: a review. Inflamm Bowel Dis 2006; 12: $1175-1184$

679 Oxelmark L, Magnusson A, Lofberg R et al. Group-based intervention program in inflammatory bowel disease patients: effects on quality of life. Inflamm Bowel Dis 2007; 13: 182-190 
680 Milne B, Joachim G, Niedhardt J. A stress management programme for inflammatory bowel disease patients. J Adv Nurs 1986; 11: 561-567

681 Schwarz SP, Blanchard EB. Evaluation of a psychological treatment for inflammatory bowel disease. Behav Res Ther 1991; 29: 167-177

682 Elsenbruch S, Langhorst J, Popkirowa K et al. Effects of mind-body therapy on quality of life and neuroendocrine and cellular immune functions in patients with ulcerative colitis. Psychother Psychosom 2005; 74: 277-287

683 Langhorst J, Mueller T, Luedtke $R$ et al. Effects of a comprehensive lifestyle modification program on quality-of-life in patients with ulcerative colitis: a twelve-month follow-up. Scand J Gastroenterol 2007; 42: 734-745

684 Szigethy E, Kenney E, Carpenter J et al. Cognitive-behavioral therapy for adolescents with inflammatory bowel disease and subsyndromal depression. J Am Acad Child Adolesc Psychiatry 2007; 46: 1290-1298

685 Mawdsley JE, Jenkins DG, Macey MG et al. The effect of hypnosis on systemic and rectal mucosal measures of inflammation in ulcerative colitis. Am J Gastroenterol 2008; 103: 1460-1469

686 Mikocka-Walus AA, Turnbull DA, Moulding NT et al. Antidepressants and inflammatory bowel disease: a systematic review. Clin Pract Epidemiol Ment Health 2006; 2: 24

687 Engstrom I. Inflammatory bowel disease in children and adolescents: mental health and family functioning. J Pediatr Gastroenterol Nutr 1999; 28: S28-33

688 Engstrom I, Lindquist BL. Inflammatory bowel disease in children and adolescents: a somatic and psychiatric investigation. Acta Paediatr Scand 1991; 80: 640-647

689 Burke PM, Kocoshis S, Neigut D et al. Maternal psychiatric disorders in pediatric inflammatory bowel disease and cystic fibrosis. Child Psychiatry Hum Dev 1994; 25: 45-52

690 Szigethy E, Levy-Warren A, Whitton S et al. Depressive symptoms and inflammatory bowel disease in children and adolescents: a crosssectional study. J Pediatr Gastroenterol Nutr 2004; 39: 395-403

691 Mackner LM, Crandall WV. Long-term psychosocial outcomes reported by children and adolescents with inflammatory bowel disease. Am J Gastroenterol 2005; 100: 1386-1392

692 Mackner LM, Crandall WV, Szigethy EM. Psychosocial functioning in pediatric inflammatory bowel disease. Inflamm Bowel Dis 2006; 12 : 239-244

693 Szigethy E, Craig AE, Iobst EA et al. Profile of depression in adolescents with inflammatory bowel disease: implications for treatment. Inflamm Bowel Dis 2009; 15: 69-74

694 Loonen HJ, Grootenhuis MA, Last BF et al. Quality of life in paediatric inflammatory bowel disease measured by a generic and a diseasespecific questionnaire. Acta Paediatr 2002; 91: 348-354

695 van der Zaag-Loonen HJ, Grootenhuis MA, Last BF et al. Coping strategies and quality of life of adolescents with inflammatory bowel disease. Qual Life Res 2004; 13: 1011-1019

696 De Boer M, Grootenhuis M, Derkx B et al. Health-related quality of life and psychosocial functioning of adolescents with inflammatory bowel disease. Inflamm Bowel Dis 2005; 11: 400-406

$697 \mathrm{Kunz} J H$, Hommel KA, Greenley RN. Health-related quality of life of youth with inflammatory bowel disease: a comparison with published data using the PedsQL 4.0 generic core scales. Inflamm Bowel Dis 2010; 16: 939-946
698 Greenley RN, Hommel KA, Nebel J et al. A Meta-analytic Review of the Psychosocial Adjustment of Youth with Inflammatory Bowel Disease. J Pediatr Psychol 2010; 35: 857-869

699 Vaisto T, Aronen ET, Simola P et al. Psychosocial symptoms and competence among adolescents with inflammatory bowel disease and their peers. Inflamm Bowel Dis 2010; 16: 27-35

700 Karwowski CA, Keljo D, Szigethy E. Strategies to improve quality of life in adolescents with inflammatory bowel disease. Inflamm Bowel Dis 2009; 15: 1755-1764

701 Shepanski MA, Hurd LB, Culton $K$ et al. Health-related quality of life improves in children and adolescents with inflammatory bowel disease after attending a camp sponsored by the Crohn's and Colitis Foundation of America. Inflamm Bowel Dis 2005; 11: 164-170

702 Joos S, Rosemann T, Szecsenyi J et al. Use of complementary and alternative medicine in Germany - a survey of patients with inflammatory bowel disease. BMC Complement Altern Med 2006; 6: 19

703 Langhorst J, Anthonisen IB, Steder-Neukamm U et al. Amount of systemic steroid medication is a strong predictor for the use of complementary and alternative medicine in patients with inflammatory bowel disease: results from a German national survey. Inflamm Bowel Dis 2005; 11 : 287-295

704 Langhorst J, Anthonisen IB, Steder-Neukamm U et al. Patterns of complementary and alternative medicine (CAM) use in patients with inflammatory bowel disease: perceived stress is a potential indicator for CAM use. Complement Ther Med 2007; 15: 30-37

705 Hanai $H$, Iida T, Takeuchi $K$ et al. Curcumin maintenance therapy for ulcerative colitis: randomized, multicenter, double-blind, placebocontrolled trial. Clin Gastroenterol Hepatol 2006; 4: 1502-1506

706 Fernandez-Banares F, Hinojosa J, Sanchez-Lombrana JL et al. Randomized clinical trial of Plantago ovata seeds (dietary fiber) as compared with mesalamine in maintaining remission in ulcerative colitis. Spanish Group for the Study of Crohn's Disease and Ulcerative Colitis (GETECCU). Am J Gastroenterol 1999; 94: 427-433

707 Joos S, Wildau N, Kohnen R et al. Acupuncture and moxibustion in the treatment of ulcerative colitis: a randomized controlled study. Scand J Gastroenterol 2006; 41: 1056-1063

$708 \mathrm{Mu}$ JP, Wu HG, Zhang ZQ et al. Meta-analysis on acupuncture and moxibustion for treatment of ulcerative colitis. Zhongguo Zhen Jiu 2007 27: 687-690

709 Summers RW, Elliott DE, UrbanJr JF et al. Trichuris suis therapy for active ulcerative colitis: a randomized controlled trial. Gastroenterology 2005; 128: 825-832

710 Chen ZS, Nie ZW, Sun QL. Clinical study in treating intractable ulcerative colitis with traditional Chinese medicine. Zhongguo Zhong Xi Y Jie He Za Zhi 1994; 14: 400-402

711 Huber R, Ditfurth AV, Amann F et al. Tormentil for active ulcerative colitis: an open-label, dose-escalating study. J Clin Gastroenterol 2007; 41: 834-838

712 Gupta I, Parihar A, Malhotra P et al. Effects of gum resin of Boswellia serrata in patients with chronic colitis. Planta Med 2001; 67: 391395

713 Gupta I, Parihar A, Malhotra P et al. Effects of Boswellia serrata gum resin in patients with ulcerative colitis. Eur J Med Res 1997; 2: 37-43

714 Langmead L, Feakins RM, Goldthorpe S et al. Randomized, doubleblind, placebo-controlled trial of oral aloe vera gel for active ulcerative colitis. Aliment Pharmacol Ther 2004; 19: 739-747 